SAND99-1290

Unlimited Release

Printed June 1999

\title{
Final REPORT ON THE OPERATION AND MAINTENANCE IMPROVEMENT PROGRAM FOR Concentrating Solar Power Plants
}

\author{
Gilbert E. Cohen \\ KJC Operating Company \\ 41100 Highway 395 \\ Boron, California 93516 \\ David W. Kearney \\ Kearney \& Associates \\ 14022 Condessa Drive \\ Del Mar, California 92014 \\ Gregory J. Kolb \\ Sandia National Laboratories \\ Solar Thermal Technology Department \\ P.O. Box 5800 \\ Albuquerque, New Mexico 87185-0435
}

\begin{abstract}
This report describes the results of a six-year, $\$ 6.3$ million project to reduce operation and maintenance (O\&M) costs at power plants employing concentrating solar power (CSP) technology. Sandia National Laboratories teamed with KJC Operating Company to implement the O\&M Improvement Program. O\&M technologies developed during the course of the program were demonstrated at the 150-MW Kramer Junction solar power park located in Boron, California. Improvements were made in the following areas: (a) efficiency of solar energy collection, (b) O\&M information management, (c) reliability of solar field flow loop hardware, (d) plant operating strategy, and (e) cost reduction associated with environmental issues. A 37\% reduction in annual O\&M costs was achieved. Based on the lessons learned, an optimum solarfield O\&M plan for future CSP plants is presented. Parabolic trough solar technology is employed at Kramer Junction. However, many of the O\&M improvements described in the report are also applicable to CSP plants based on solar power tower or dish/engine concepts.
\end{abstract}




\section{Acknowledgments}

The following team successfully implemented this project:

Amar Amarnani (KJCOC)

Pam Baca (KJCOC)

Avi Brenmiller (SOLEL)

Robert Cable (KJCOC)

Gilbert Cohen (KJCOC, now with Duke Solar)

John Costanzo (KJCCC)

Chuck Damron (KJCOC)

Vern Dudley (SNL, retired)

Lisa Fimbres (KJCOC)

Robert Fimbres (KJCOC)

Fred Foster (KJCOC)

Scott Frier (KJCOC)

Randy Gee (IST)

Michael Geyer (PSA)

Janis Hill (KJCOC)

Karen Holifield (KJCOC)

David Kearney (K\&A)

Greg Kolb (SNL)

Glen Lane (KJCOC)
Frank Lippke (SNL, now with VALEO)

Rod Mahoney (SNL)

Tom Mancini (SNL)

Ken May (IST)

Paul Nava (Pilkington)

Scott Nolan (KJCOC)

Hank Price (NREL)

Nicholas Potrovitza (KJCOC)

David Rib (KJCOC)

Mike Roberson (KJCOC)

David Russitto (ATS)

Jacob Shemesh

Michael Sloan (VERA)

Harvey Stephens (KJCOC)

John Taylor (KJCOC)

Bill Tomlinson (KJCOC)

Craig Tyner (SNL)

Ed Vanderberg (KJCOC)

Ronny Wise (KJCOC) 


\section{DISCLAIMER}

This report was prepared as an account of work sponsored by an agency of the United States Government. Neither the United States Government nor any agency thereof, nor any of their employees, make any warranty, express or implied, or assumes any legal liability or responsibility for the accuracy, completeness, or usefulness of any information, apparatus, product, or process disclosed, or represents that its use would not infringe privately owned rights. Reference herein to any specific commercial product, process, or service by trade name, trademark, manufacturer, or otherwise does not necessarily constitute or imply its endorsement, recommendation, or favoring by the United States Government or any agency thereof. The views and opinions of authors expressed herein do not necessarily state or reflect those of the United States Government or any agency thereof. 


\section{DISCLAIMER}

Portions of this document may be illegible in electronic image products. Images are produced from the best available original document. 


\section{Contents}

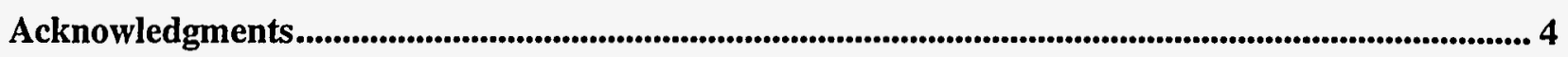

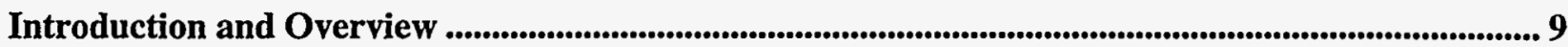

Improving the Efficiency of Solar Energy Collection ..................................................................................... 11

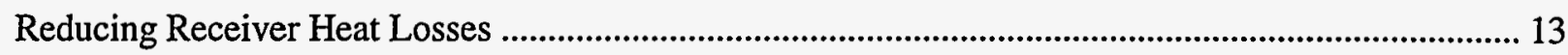

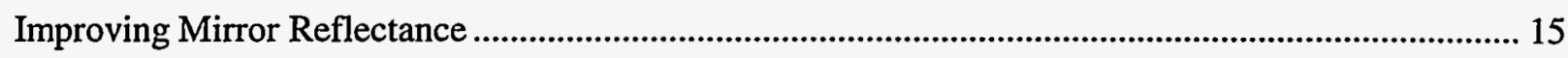

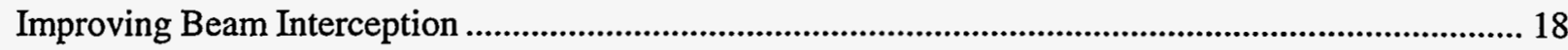

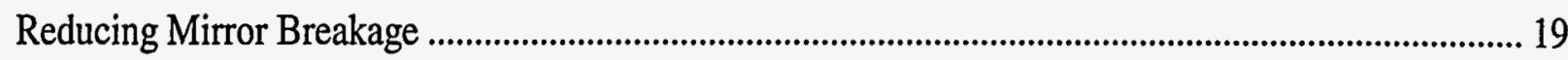

Improving O\&M Information Management within a Solar Power Park............................................... 20

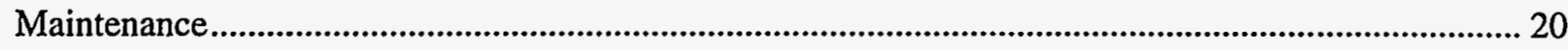

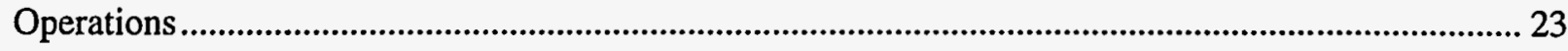

Improving the Reliability of Solar Field Flow-Loop Hardware................................................................ 24

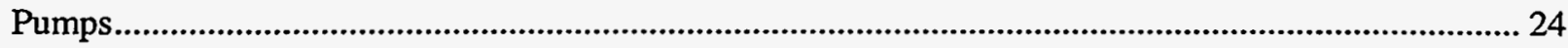

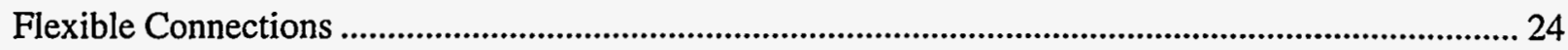

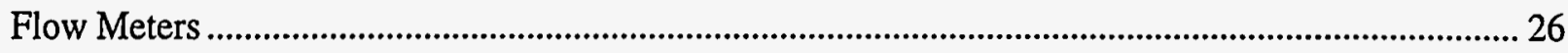

Developing an Optimum Plant Operating Strategy .................................................................................... 26

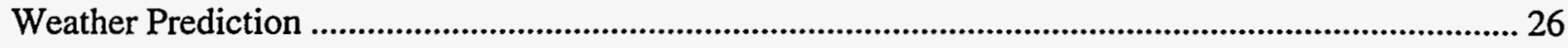

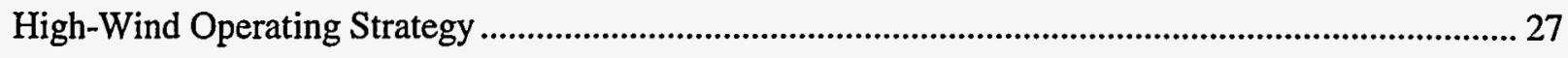

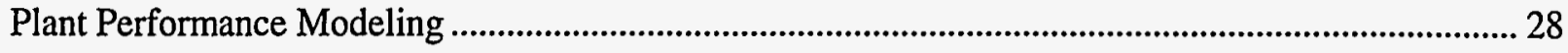

Reducing Costs Associated with Environmental Issues....................................................................... 30

Volatile Organic Compounds Emissions and Oil Spills ................................................................... 30

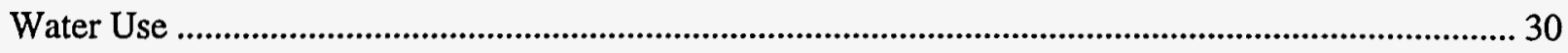

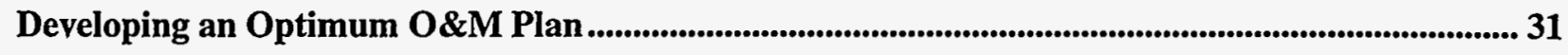

Comparing O\&M Costs Before and After the O\&MIP .......................................................................... 32

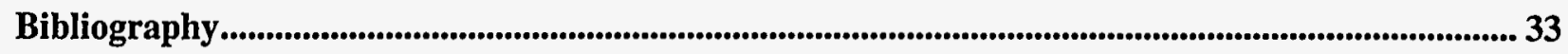




\section{Appendices}

Appendix A Testing of the LS-2 Trough Collector at Sandia National Laboratories....... 37

Appendix B Heat Collection Element Refurbishment.................................................... 43

Appendix C Heat Collection Element Development................................................... 45

Appendix D Vacuumless Heat Collection Element.................................................... 55

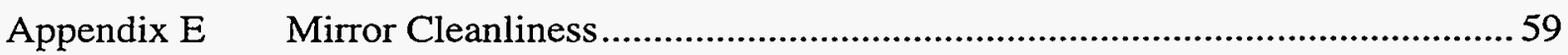

Appendix F Torque Tube Alignment .......................................................................6 67

Appendix G $\quad$ LS-3 Collector Loop Testing.............................................................. 71

Appendix $\mathrm{H} \quad$ Strengthened Mirrors............................................................................... 81

Appendix I $\quad$ Power Park Data Network .................................................................... 87

Appendix J Intertie of Plant Controls to Power Park Data Network ............................. 93

Appendix K Computer Maintenance Management System........................................... 99

Appendix L Solar Field Maintenance Planning ................................................... 103

Appendix M Remote Data Entry to Solar Field Maintenance Database ........................ 107

Appendix N Computerized Drawing and Document Control...................................... 111

Appendix $\mathrm{O} \quad$ Computer-Based Operator Log .............................................................. 115

Appendix P Heat Transfer Fluid Pump Upgrade ...................................................... 119

Appendix Q Comparative Testing of Flexible Hoses versus Rotating Joints................ 121

Appendix R Heat Transfer Fluid Flow Measurement ................................................ 125

Appendix S Weather Prediction to Support Operation and Maintenance Planning....... 129

Appendix T High-Wind Operating Strategy....................................................... 131

Appendix U Solar Field Flow Control ..................................................................... 135

Appendix V Analytical Model of Solar Collector Field ............................................... 141

Appendix W Hourly Performance Model................................................................... 149

Appendix X On-Line Performance Monitoring and Optimization ............................. 157

Appendix Y Weather Station Data Management........................................................ 161

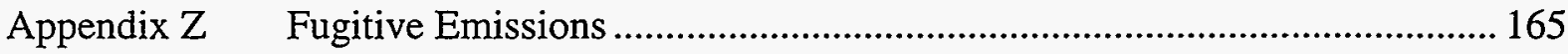

Appendix AA Water Use Study........................................................................ 167

Appendix AB Recommended Operation and Maintenance Plan .................................. 173

Distribution 


\section{Acronyms}

CMMS Computer Maintenance Management System

CSP concentrating solar power

EPA Environmental Protection Agency

FSP Field Status Program

HCE heat collection element

HTF heat transfer fluid

KJCOC KJC Operating Company

$\mathrm{kW} \quad$ kilowatt

$\mathrm{m}^{2} \quad$ square meter

MOV motor-operated valve

MPAC Maintenance Planning and Control

MW megawatt

O\&M operation and maintenance

O\&MIP Operation and Maintenance Improvement Program

OPM On-line Performance Monitoring

SCA solar collector assembly

SEGS solar electric generating systems

SNL Sandia National Laboratories

VOC volatile organic compound 


\section{FINAL REPORT ON THE OPERATION AND MAINTENANCE IMPROVEMENT PROGRAM For Concentrating Solar Power Plants}

\section{Introduction and Overview}

This report presents the results of a six-year, $\$ 6.3$ million project to reduce operation and maintenance (O\&M) costs at power plants employing concentrating solar power (CSP) technology. Sandia National Laboratories (SNL) teamed with KJC Operating Company (KJCOC), the O\&M company that runs the plant, to implement this O\&M Improvement Program (O\&MIP) on a 50-50 cost-shared basis. O\&M technologies developed during the course of the program were demonstrated at the 150-MW Kramer Junction solar power park located in Boron, California. The Kramer Junction power park is comprised of five 30-MW plants that were built by LUZ International Ltd. in the late 1980s. These plants are classified as hybrid-solar plants because the solar fields are paralleled with a gas-fired boiler, and the technology is known as solar electric generating systems (SEGS). While all plants often run at full output in solar-only mode, they are allowed by law to use up to $25 \%$ of the annual energy input from fossil energy (see Table 1 and Figure 1).

At the inception of the program, O\&M requirements at Kramer Junction comprised $25 \%$ of total electricity costs. The goal of the O\&MIP was to develop improved management methods and O\&M technologies that would approximately halve the O\&M contribution to electricity costs, expressed in cents per kilowatt hour $(\phi / \mathrm{kWh})$ in future CSP plants. Program tasks focused on improving performance, increasing reliability, upgrading control systems, reducing maintenance costs (through more effective planning and implementation), increasing the efficiency and lifetime of the solar field components, improving the effectiveness of the power block/solar field interface, and a variety of other measures that either decrease costs, improve electrical output, or both.

Table 1. Basic Characteristics of the SEGS Plants at Kramer Junction

\begin{tabular}{|c|c|c|c|c|c|}
\hline Plant & $\begin{array}{c}\text { Startup } \\
\text { Year }\end{array}$ & $\begin{array}{c}\text { Capacity } \\
\text { (net) }\end{array}$ & $\begin{array}{c}\text { Solar Field } \\
\text { Temperature }\end{array}$ & $\begin{array}{c}\text { Collector } \\
\text { Technology }\end{array}$ & $\begin{array}{c}\text { Solar Field } \\
\text { Size }\end{array}$ \\
\hline III & 1987 & $30 \mathrm{MW}$ & $349^{\circ} \mathrm{C}$ & LS-2 & $230,300 \mathrm{~m}^{2}$ \\
\hline IV & 1987 & $30 \mathrm{MW}$ & $349^{\circ} \mathrm{C}$ & LS-2 & $230,300 \mathrm{~m}^{2}$ \\
\hline V & 1988 & $30 \mathrm{MW}$ & $349^{\circ} \mathrm{C}$ & LS-2/LS-3 & $250,560 \mathrm{~m}^{2}$ \\
\hline VI & 1988 & $30 \mathrm{MW}$ & $390^{\circ} \mathrm{C}$ & LS-2 & $188,000 \mathrm{~m}^{2}$ \\
\hline VII & 1989 & $30 \mathrm{MW}$ & $390^{\circ} \mathrm{C}$ & LS-2/LS-3 & $194,280 \mathrm{~m}^{2}$ \\
\hline
\end{tabular}




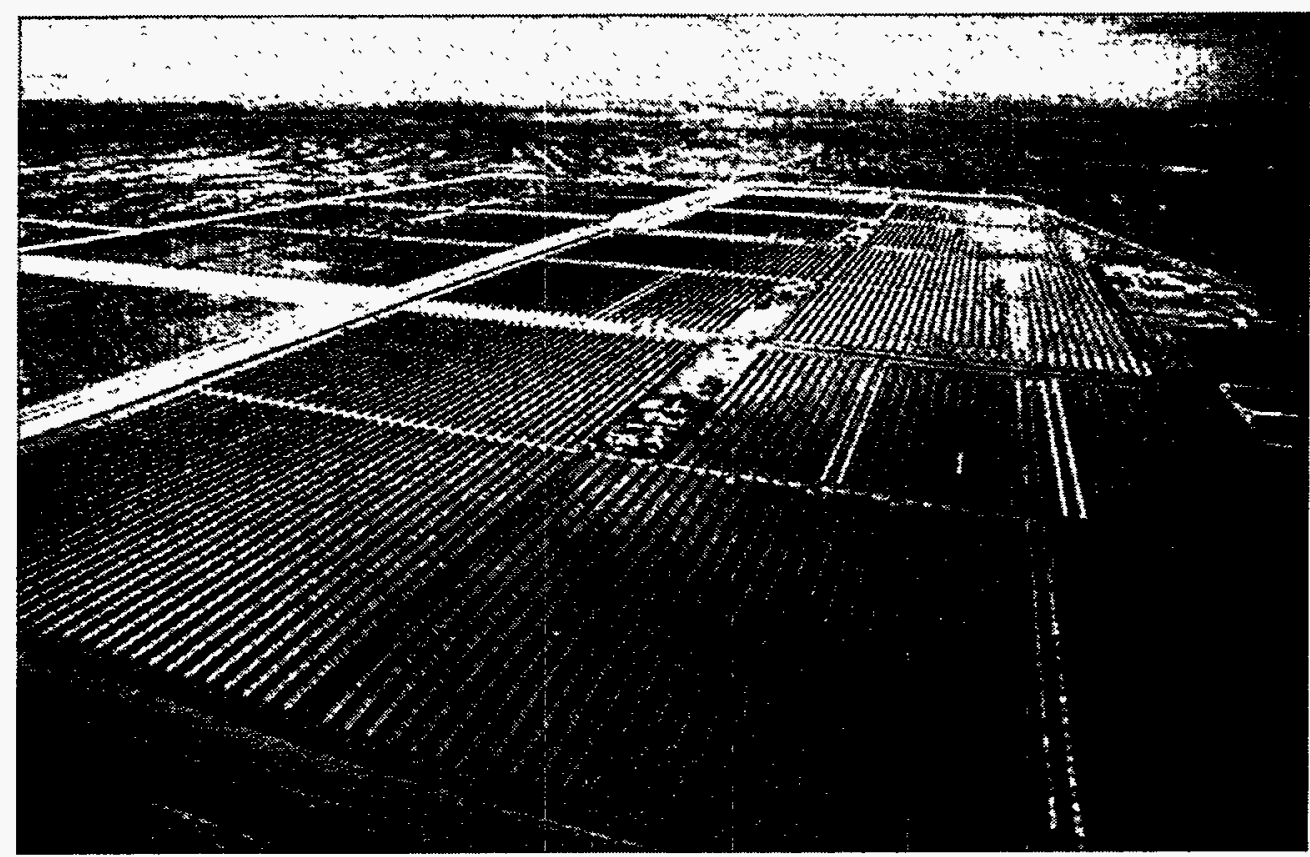

Figure 1. The Kramer Junction power park is comprised of five 30-MW plants.

The specific areas targeted for O\&M cost reduction were selected in a quasi-scientific manner. An initial list was created by examining historical O\&M cost and performance data at Kramer Junction. For example, this identified components that were exhibiting much higher than expected failure rates and replacement costs. KJCOC's "lost-energy" analysis was also examined, which helped identify plant components that were not meeting their performance goals. In addition, for ideas not backed by historical data, expert judgment was used to estimate the costreduction potential. With all ideas on the table, we then rank-ordered them by payback potential.

The work in this program covered only development and prototype costs in selected plants, not full implementation on all the Kramer Junction plants. The full value of the technology developed in the O\&MIP will be realized in new plants only where new methods, design improvements, and component upgrades can be implemented from the beginning. However, in some, though not all, cases, the plant owners made the decision to fully implement new improvements. In addition, other nonprogram improvements in plant condition and O\&M practices were being implemented in parallel during the course of the program work. As a result of these influences, we were able to achieve close to a $40 \%$ reduction in O\&M costs for the Kramer Junction power park. This reduction is equivalent to a $\$ 4$ million annual savings, which means that funds expended in the program will be paid back after approximately $1 \frac{1}{2}$ years of power park operation.

In this report, we will present the highlights of the O\&M technology and methods we implemented during the program and quantify the overall cost reduction we achieved at Kramer Junction. For purposes of discussion, the topics are grouped in the following manner:

- Improving the efficiency of solar energy collection

- Improving O\&M information management 
- Improving the reliability of solar field flow-loop hardware

- Developing an optimum plant operating strategy

- Reducing costs associated with environmental issues

- Developing an optimum O\&M plan for future plants

- Comparing costs before and after the O\&MIP

To learn more about a particular item, refer to an expanded discussion in the identified appendices.

While most tasks led to improvements, not everything we tried was successful. We will describe our successes and failures and suggest areas for future development.

It is important to note that even though parabolic trough CSP technology is used at Kramer Junction, many of the O\&M improvements described herein are also applicable to CSP plants based on solar power tower or dish/engine concepts.

\section{Improving the Efficiency of Solar Energy Collection}

The five 30-MW plants at Kramer Junction use large fields of parabolic trough solar collector assemblies (SCAs) to supply thermal energy to produce superheated steam to drive a Rankine steam turbine/generator cycle. The design and configuration of the power block and balance-ofplant systems are routine. A typical 30-MW system schematic is shown in Figure 2.

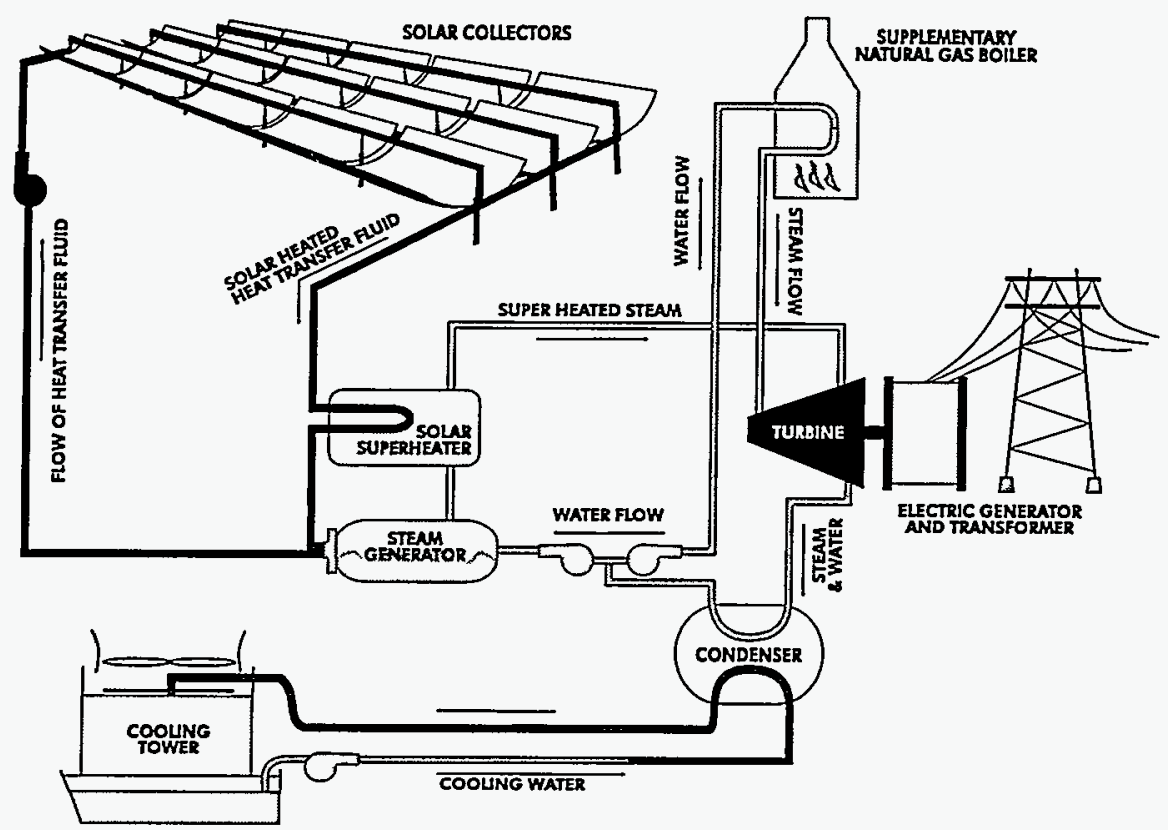

Figure 2. Flow schematic for a typical 30-MW plant. 
Each of the approximately 1,000 SCAs per 30-MW plant has silvered low-iron glass reflectors that focus direct solar radiation on an efficient evacuated receiver, or heat collection element (HCE). Each SCA has the capability to track the sun independently from the remainder of the SCAs in the solar field. Two generations of the SCAs (LS-2 and LS-3) are installed at Kramer Junction. Table 2 highlights the main characteristics of the two generations of collectors. Good performance of these solar fields is necessary to maximize electricity production at the plants.

Many factors affect the performance of the solar field. To gain a clear understanding of these factors, extensive testing was performed on specially prepared test loops at SNL and at Kramer Junction. The factors that were targeted for improvement within the O\&MIP are those discussed in the paragraphs that follow.

Table 2. Specifications for Parabolic Trough Solar Collector Assemblies

\begin{tabular}{|l|c|c|}
\hline & LS-2 & LS-3 \\
\hline \multicolumn{2}{|c|}{ Solar Collector Assembly } \\
\hline Aperture Area (m ${ }^{2}$ ) & 235 & 545 \\
Aperture (m) & 5.0 & 5.76 \\
Length (m) & 47.1 & 95.2 \\
Solar Concentration Ratio & 71 & 82 \\
Mirror Reflectance (clean) & 0.94 & 0.94 \\
Optical Efficiency* & 0.737 & 0.80 \\
\hline \multicolumn{2}{|c|}{ Heat Collection Element } \\
\hline Pipe Diameter (m) & 0.070 & 0.070 \\
Length (m) & 4 & 4 \\
Glass Annulus Diameter (m) & 0.115 & 0.115 \\
HCEs per SCA & 12 & 24 \\
Selective Surface & $10^{-4}$ & Cermet \\
Annulus Vacuum (torr) & Black Chrome and Cermet & $10^{-4}$ \\
\hline \multicolumn{2}{|c|}{ Heat Collection Element Optical Properties } \\
\hline Annulus Transmittance & 0.95 & 0.95 \\
HCE Absorptance & 0.95 & 0.96 \\
HCE Emittance & $0.24 / 0.19$ \\
at Temperature ( $\left.{ }^{\circ} \mathrm{C}\right)$ & 300 & 0.19 \\
\hline
\end{tabular}

*SNL validated the optical efficiency for the LS-2. See Appendix A. 


\section{Reducing Receiver Heat Losses}

To keep thermal losses in the solar field at a minimum and the plant electrical output at a maximum, the vacuum within the HCE glass annulus should ideally be maintained at the design value of $10^{-4}$ torr. However, HCEs can lose vacuum because of a crack or leak in the annulus. The glass can break because of poor alignment during installation, O\&M accidents, or physical interference from other components, such as a mirror breaking in the wind and falling on the HCE.

At the beginning of the O\&MIP, approximately $15 \%$ of the 50,000 HCEs at Kramer Junction had lost their vacuum, and a significant portion of these had completely lost the glass envelope. HCE replacement was estimated to be expensive and $\mathrm{KJCOC}$ wanted to be sure the replacement cost was justified. Consequently, SNL performed a detailed measurement of collector efficiency and HCE thermal losses at its rotating platform test facility in Albuquerque, New Mexico (see Figures 3 and 4). The efficiency of an LS-2 collector segment containing two HCEs was measured given a variety of conditions: vacuum intact, vacuum lost, glass envelope missing, high winds, and so forth (see Appendix A). Although test results confirmed that maintaining vacuum is important, the results showed reasonable performance with the vacuum lost but with the glass envelope intact. However, if the glass envelope is missing, heat loss becomes excessive, especially during windy weather.

Using the results of the SNL tests, KJCOC performed an economic analysis and determined that breakage of the glass envelopes was the most important cause of energy and revenue loss in the solar fields at the power plant. KJCOC therefore decided to replace all HCEs that had broken glass envelopes. From a maintenance logistics point of view, it makes sense to replace HCEs only when several adjacent ones are bad. Thus, the O\&MIP developed a method of in situ repair of broken glass envelopes. The repair method involves installation of a split-glass sleeve (see Figure 5). Glass tubes are cut along the length of the cylinder and the two halves are held together with high-temperature adhesives and springs (see Appendix B). Upon replacement of a repaired HCE with a new one, the temporary sleeve is removed and relocated elsewhere in the solar field.

Besides maintaining good vacuum, another method of reducing heat losses from HCEs is to reduce the emittance of the selective coating on the receiver tube. During the O\&MIP, a new cermet coating was developed independently by SOLEL Solar Systems with an emittance that is approximately $50 \%$ below the value listed in Table 2 (see also Appendix C). In late 1997, HCEs with the new coating were beginning to be installed in the Kramer Junction solar fields. This advancement significantly reduces heat losses and is estimated to lower electricity costs in future trough plants by approximately $4 \%$. This new coating also opens the door to the possibility of a new low-cost HCE. Because thermal losses are lowered, a new HCE might be constructed that does not employ the vacuum feature, yet still maintains acceptable heat losses. This "vacuumless HCE" is estimated to cost approximately one-half of the previous model because the complex evacuation procedure and seals are avoided. The O\&MIP built several vacuumless prototypes (see Appendix D) and compared their thermal performance with the evacuated HCEs. While the thermal performance of the vacuumless prototypes was approximately $11 \%$ below the evacuated $\mathrm{HCE}$, there are several individuals at Kramer Junction who are strong believers in the vacuumless approach and believe that research and development should continue in this area. 


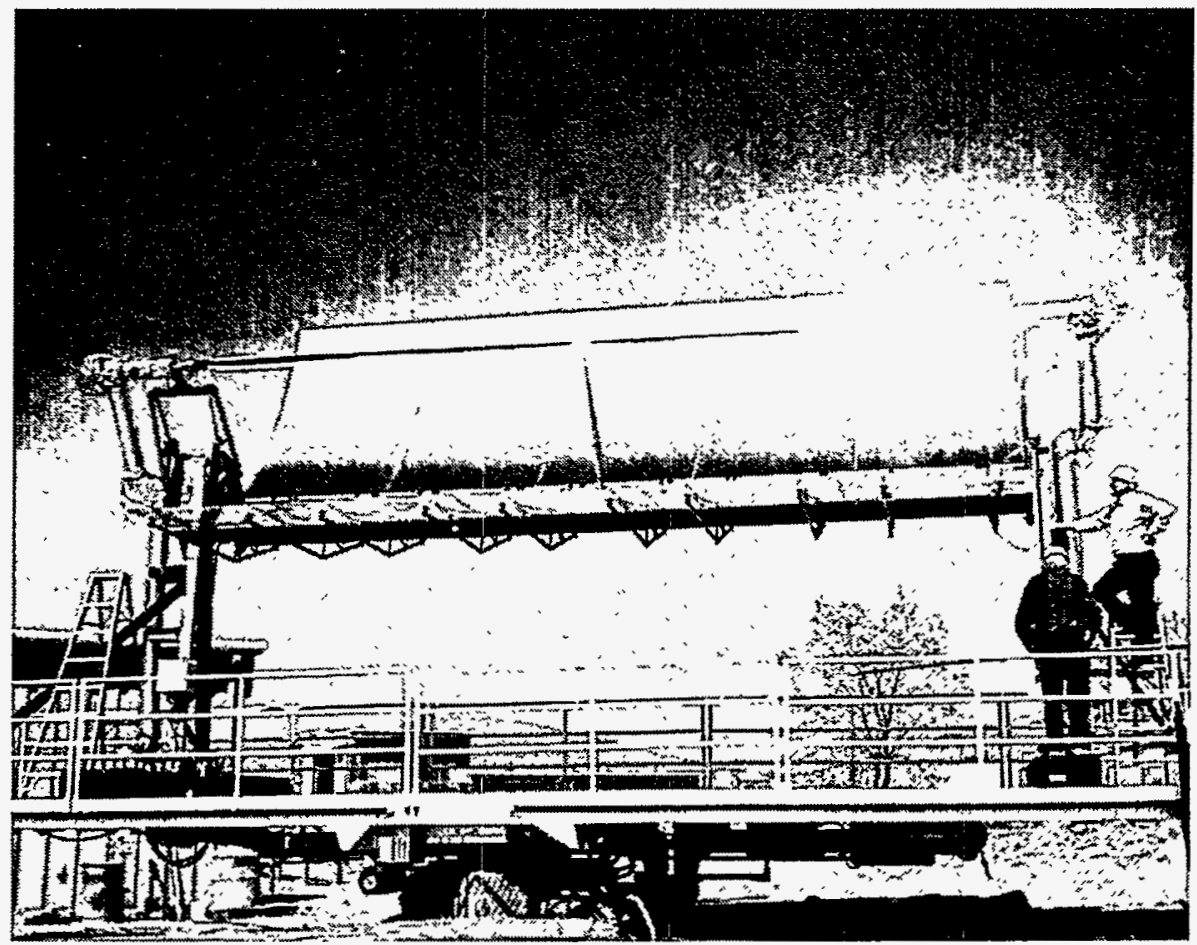

Figure 3. An LS-2 collector was tested on SNL's rotating platform. The platform allows the collector to be pointed directly at the sun at all times of the day.

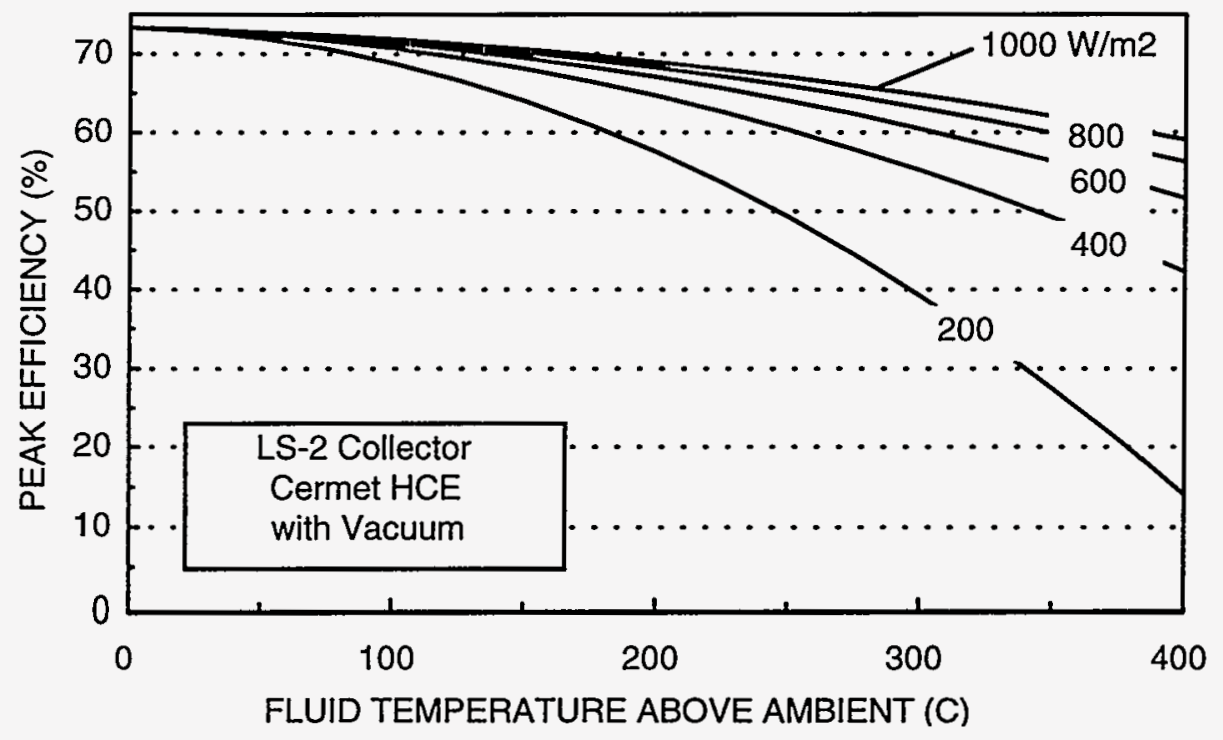

Figure 4. Test results for cermet-coated HCEs. Heat transfer fluid temperatures vary over a wide range within a collector field; thus, collection efficiency is a function of operating temperature. 


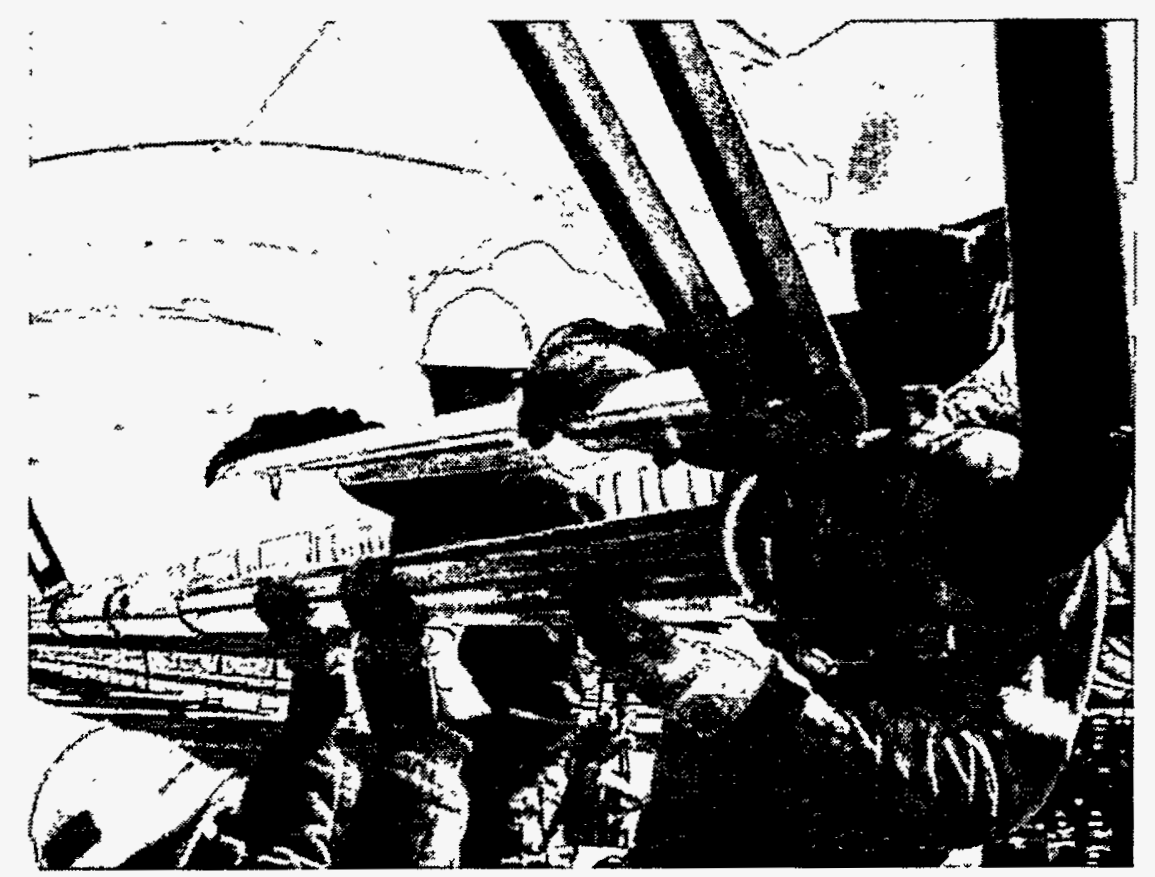

Figure 5. Split-glass envelopes were developed as a temporary method of reducing HCE heat losses.

\section{Improving Mirror Reflectance}

Solar field performance and power-sales revenue are directly dependent on mirror reflectivity. Economic analyses performed by $\mathrm{KJCOC}$ indicate it is cost-effective to maintain the average field reflectivity above $90 \%$. To accomplish this, frequent mirror washing is required. There are more than 500,000 mirror segments at Kramer Junction. Washing effectiveness varies according to mirror location (e.g., proximity to high soiling areas, such as roads or cooling towers) and time of year.

A large number of reflectivity readings are required to gather the necessary information for rational decisions on washing frequency. Before the O\&MIP, the traditional method of taking readings employed a laboratory-type instrument that was cumbersome to use and proved to be impractical for O\&M management of large solar fields. Consequently, we developed a more practical and user-friendly reflectometer (Figure 6) by modifying a device built by $\mu$ Scan Incorporated, which was previously used to measure the luster of automotive paints (see Appendix E). Using the new device, the number of data points that can be collected within a unit of time has increased by a factor of six, allowing for a much broader field survey than previously possible.

With a good knowledge of the field reflectivity, $\mathrm{KJCOC}$ was able to perform a detailed comparison of the three different washing methods. Figure 7 shows the traditional high-pressure/ low-water-volume cleaning method and Figure 8 shows the new high-pressure method developed by the O\&MIP. Figure 9 shows the traditional deluge method (low-pressure/high-water-volume). Of the three methods, the new rotating head or "Twister" approach was preferred because only one person is needed to operate the rig, and it is the most effective method of cleaning the mirrors (see Appendix E). 
The cleaning methods described above are effective in cleaning mirrors subjected to conventional desert soiling. However, mirror soiling rates near the cooling towers are particularly high because of cooling tower drift. To clean these mirrors, we applied an acid gel, followed by high pressure spraying with demineralized water (see Appendix E). While the method was effective in restoring reflectivity ( $10 \%$ to $40 \%$ before, $88 \%$ after), the method is used infrequently on only a very small portion of the field because of environmental concern over the use of acid. It is worth noting that commercially available anti-soiling agents were also applied to the mirrors near the cooling towers, but their use was discontinued because they were not deemed cost-effective.

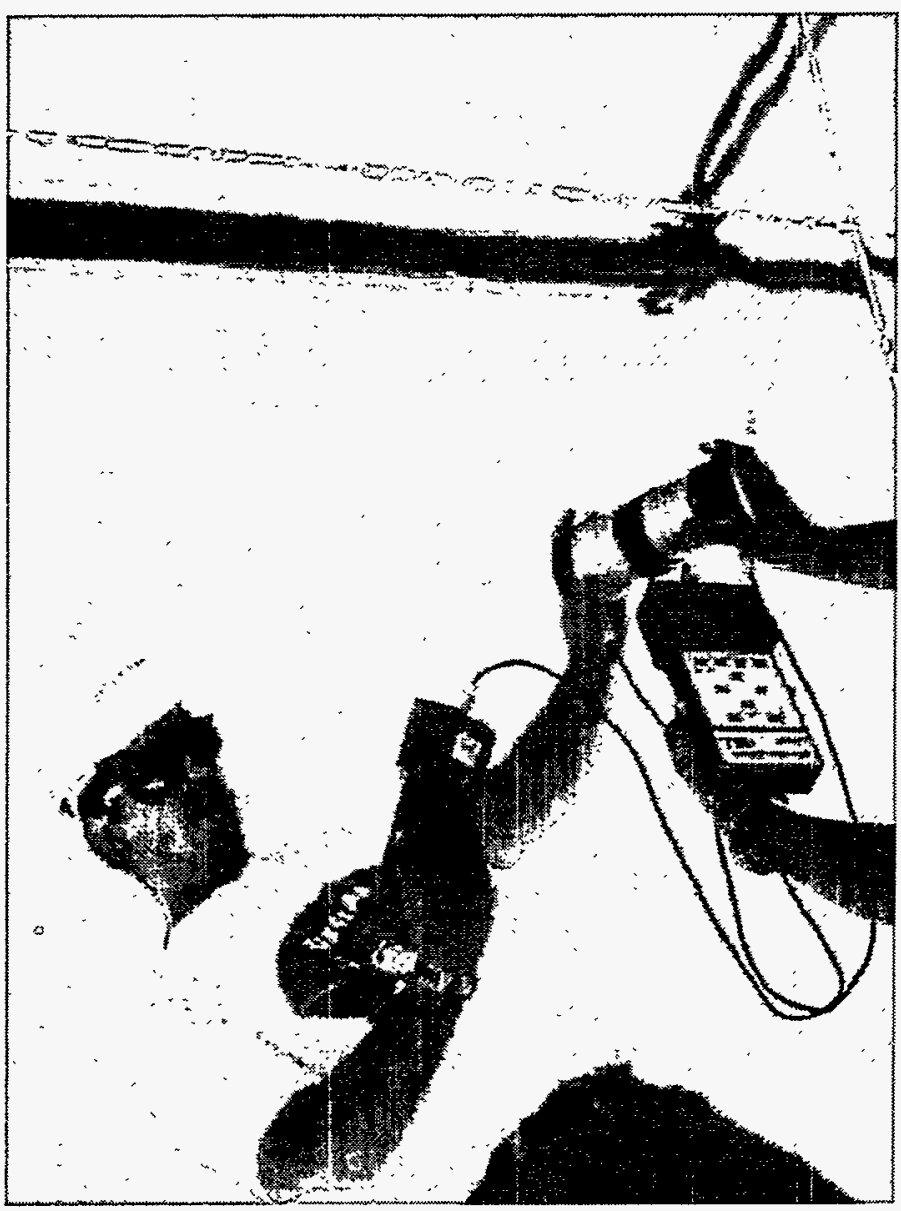

Figure 6. Reflectivity measurements are now taken with the $\mu$ Scan unit rather than the previous laboratory instrument from D\&S, Inc. 


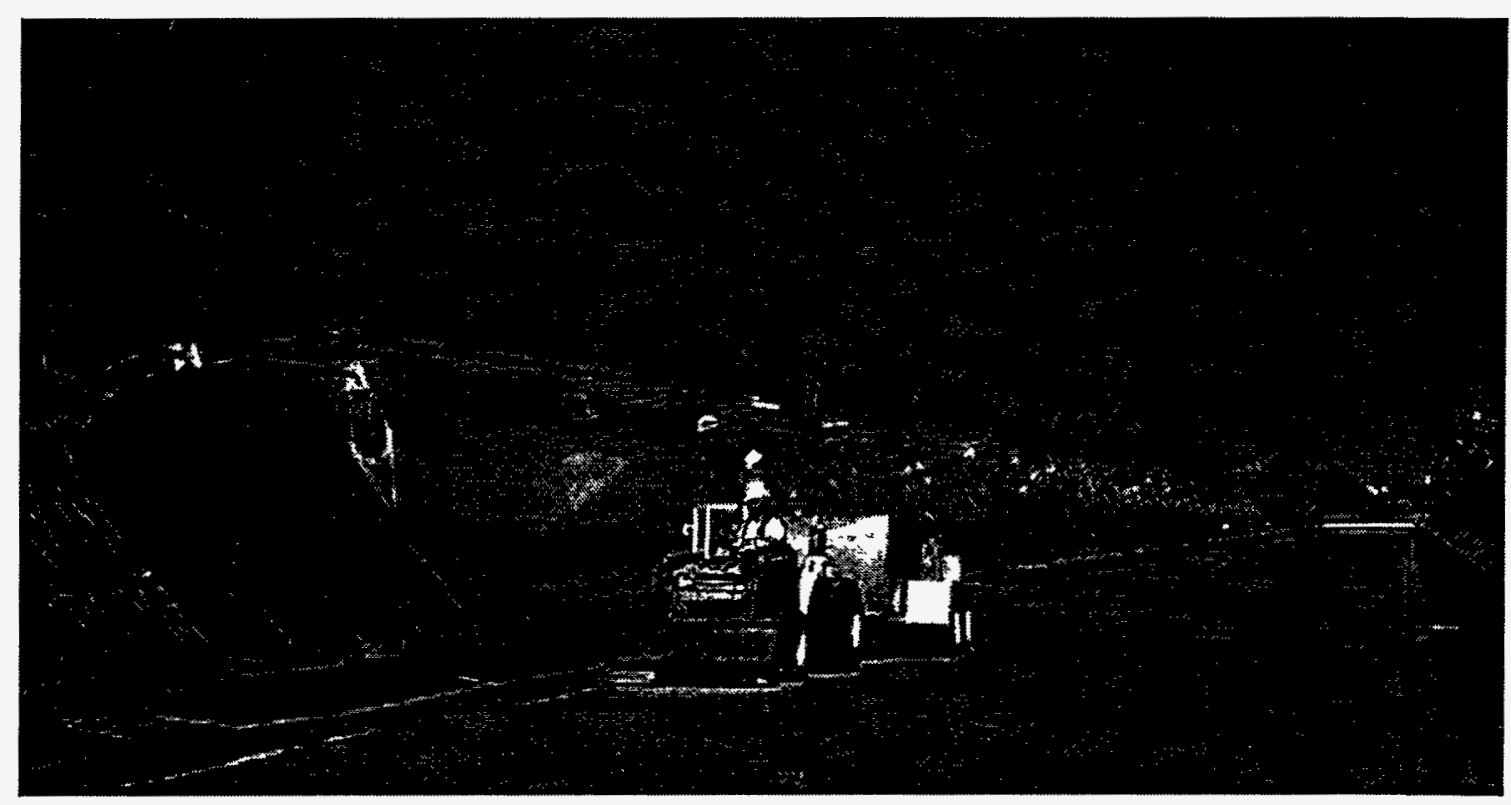

Figure 7. In the traditional method, high-pressure demineralized water is sprayed on the mirrors with hand-held nozzles.

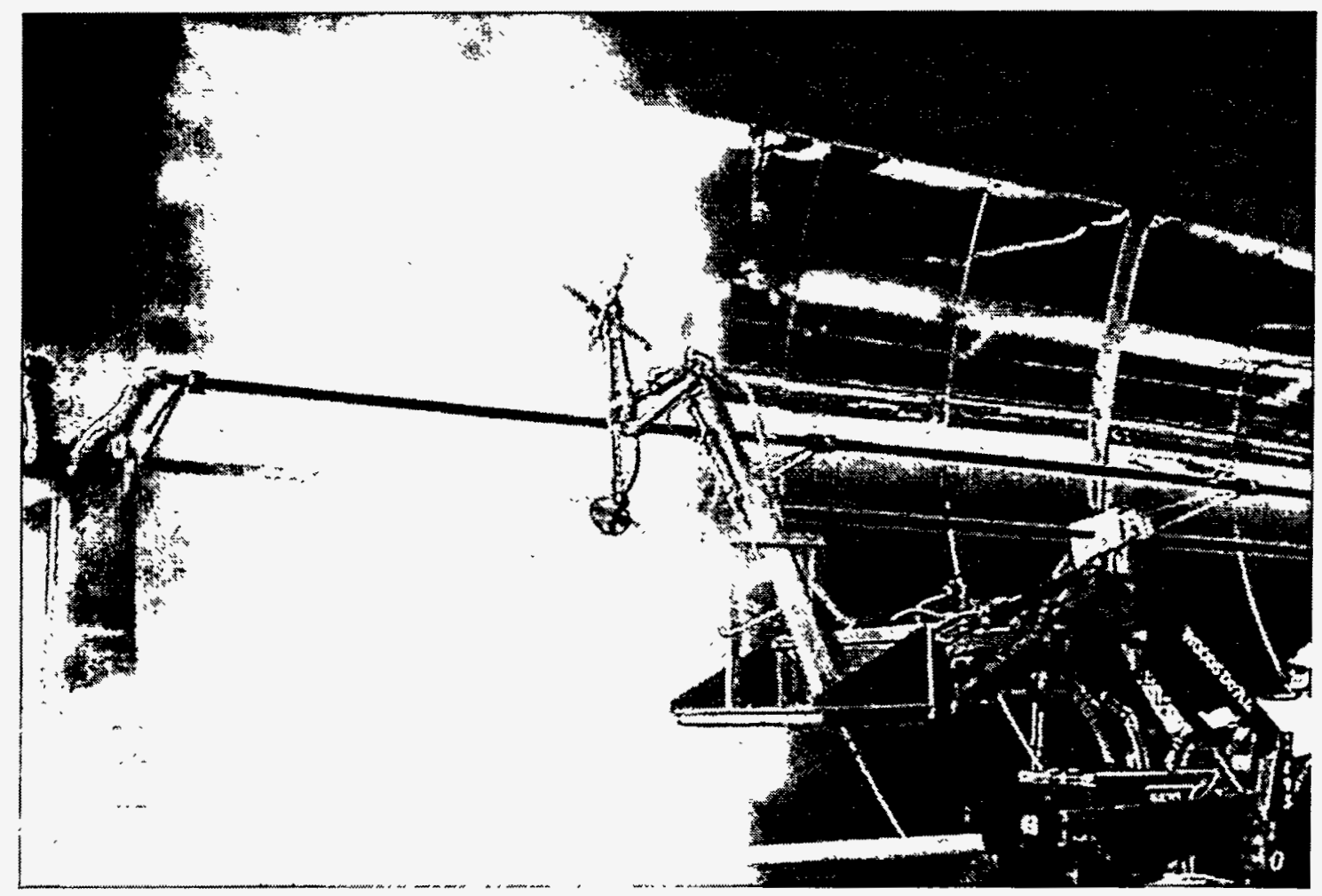

Figure 8. High-pressure demineralized water can also be sprayed on the mirrors with a new "rotating-head" rig (a.k.a. Twister) developed by the O\&MIP. 


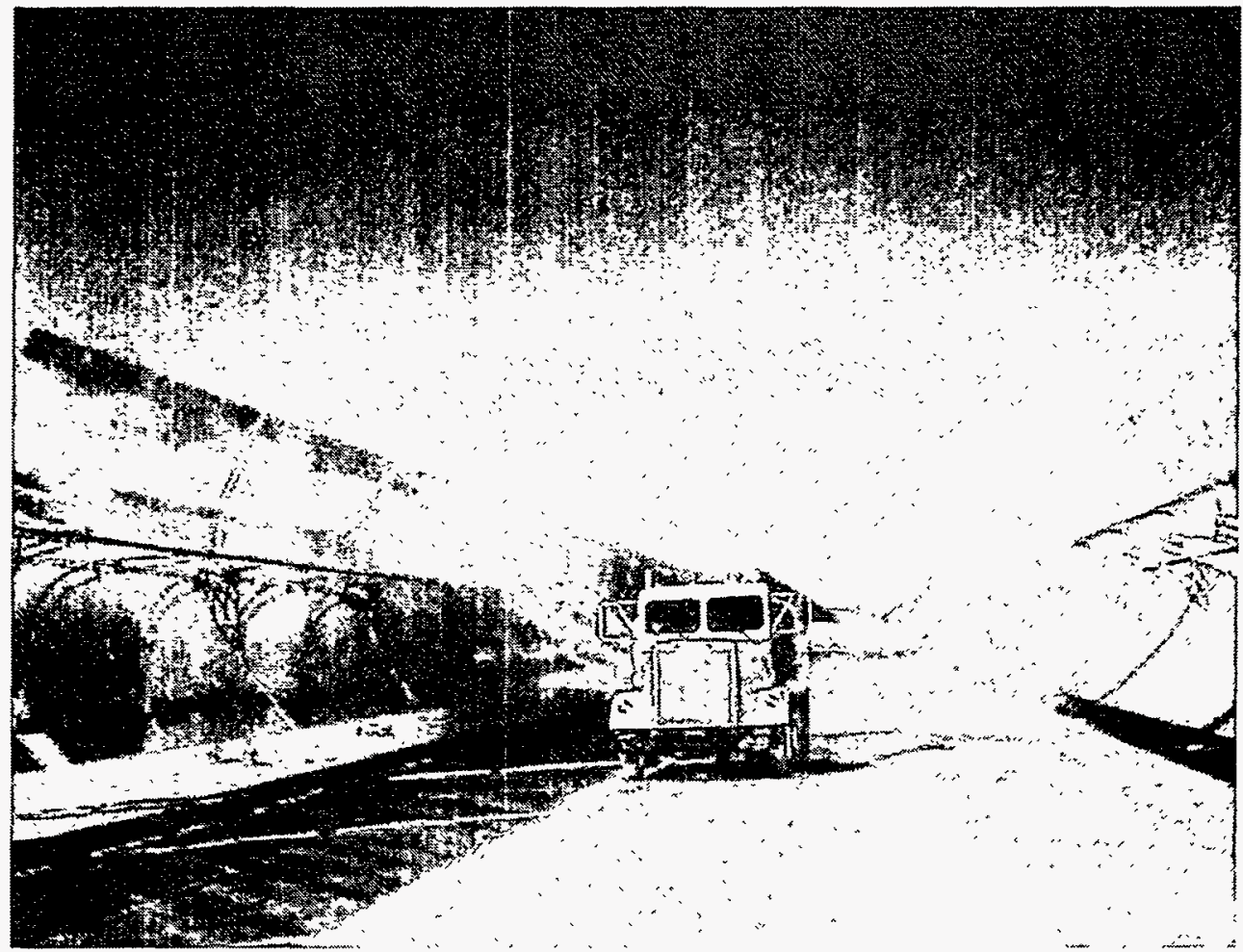

Figure 9. A truck can be used to apply a "deluge-type" stream of water to parallel rows of mirrors.

\section{Improving Beam Interception}

The performance of the solar field is strongly influenced by the ability of the collectors to focus the reflected solar beam on the receiver (HCE). An accurate focus requires excellent alignment between the tracking system, the collector structure, and the HCE. The LS-2 and LS-3 collectors at Kramer Junction were designed for a high optical accuracy, but realization of the design specifications depends on proper fabrication and installation. After installation, alignment of the collector components can deteriorate over time because of normal movement and the effects of expected structural forces (e.g., gravity, wind, and sonic booms).

Observations of the focusing accuracy of the solar field indicated an unacceptable deterioration in alignment in some of the LS-2s at SEGS IV and most of the LS-3s at SEGS VII. The fix identified for the LS-2s was to realign the torque tube portion of the mirror support structure; a new alignment procedure was implemented as part of the O\&MIP (see Appendix F). The problems with the LS-3s were deemed to be more serious and required the installation of counterweights and gearbox brakes as well as correcting the structure and sun-sensor alignment. ${ }^{1}$ To study the complex interactions of the LS-3 problems, the O\&MIP installed special test instrumentation on two parallel collector loops at SEGS VII. One of these loops, the reference loop, was left unmodified as according to the initial installation. The other loop (the test loop) was modified with combinations of proposed fixes and compared to the unmodified reference loop. In some

\footnotetext{
${ }^{1}$ Most of the problems with the LS-3 SCAs at Kramer Junction were corrected in the design and installation of the LS-3 SCAs in SEGS VIII and IX at Harper Lake, California.
} 
cases, these experiments were able to improve the collection performance of the LS-3s by as much as a factor of two (see Appendix $G$ ). This comparative technique has proved to be a very valuable and practical method of identifying the most effective fixes to collector problems. It is highly recommended that future trough plants set up similar test loops within their solar fields.

\section{Reducing Mirror Breakage}

The glass-mirror panels at Kramer Junction have exhibited excellent lifetime with excellent durability and no degradation of the silvered surface. One weak point, however, has been excess mirror breakage in high winds near the edges of the solar fields, leading to excess O\&M expenses and a reduction in the amount of solar energy collected (see Figure 10). The impact of broken mirrors can "cascade" through the breakage of HCE glass envelopes and/or other mirrors by falling pieces of a broken mirror panel. It has been observed that glass breakage from high winds is seldom experienced on "internal" collectors where wind loads are reduced.

To correct this problem, the O\&MIP attempted to develop a new strengthened mirror panel that could be installed near the edges of the field. Three separate solutions were attempted (see Appendix $\mathrm{H}$ ). In the first attempt, we installed several hundred non-glass panels composed of layered aluminum/plastic composite that used a silvered Teflon film (ECP-305+) as the reflector. While these panels showed excellent mechanical integrity during high winds, this approach was abandoned because the film became severely degraded. In the second and third attempts, we attempted to strengthen the existing glass mirrors by applying foam strips or fiberglass resins/ fabrics on the backside of the mirror. To date, it appears that the fiberglass resin/fabric approach is preferred; future plants should consider installing this type of reinforced mirror on the edges of the solar field.

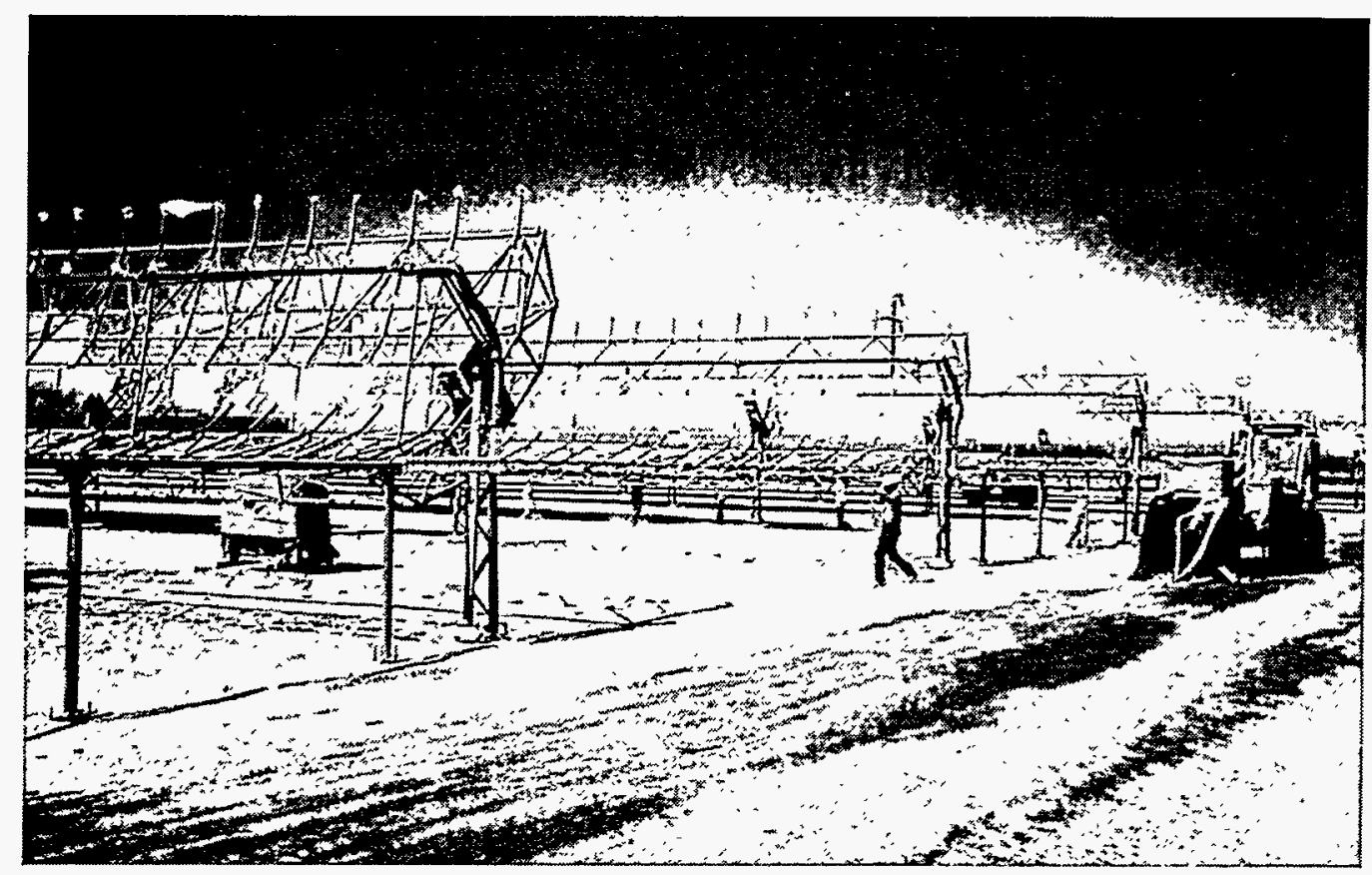

Figure 10. Mirror damage typically occurs along the edge of a solar field. In the worst-case event shown above, a tornado hit Kramer Junction on July 29, 1995. 


\section{Improving O\&M Information Management within a Solar Power Park}

The five 30-MW SEGS plants at Kramer Junction comprise a solar power park. Power parks (multiple plants at the same site $^{2}$ ) are the expected method of deploying large-scale solar power plants during the 21 st century. One of the advantages of a power park is that the O\&M crew can be shared by several plants. This sharing increases the efficiency of the O\&M organization and reduces the O\&M cost per plant. In order for this improved efficiency to be fully realized, site O\&M planners must have rapid access to maintenance and performance databases at each plant within the park and a method of quickly ordering the required work and replacement parts.

At the start of the O\&MIP, information gathering and transfer to O\&M planners was lethargic because a largely paper-based system was used and the planners had to travel long distances to communicate with the O\&M crew stationed at several control rooms, warehouses, and shops within the 700-acre site. Thus, one of the first tasks of the O\&MIP was to link the remote locations with the planning office via a fiber-optic network. Linking involved the installation of optical cable, PC workstations (see Appendix I), and replacement of some control system hardware and software (see Appendix J). The latter was required to allow communication of plant status with other devices on the data highway. With the network in place (see Figure 11), KJCOC then installed specialized software that replaced the old paper method of O\&M planning and monitoring with more efficient electronic methods, as discussed in the paragraphs that follow.

\section{Maintenance}

With the advent of the PC age, the electric power industry is relying more heavily upon a multitude of Computer Maintenance Management System (CMMS) products to streamline its O\&M planning activities. These software products bring together such activities as the master equipment list, equipment reliability histories, work order system, purchase orders, stock issue requests, workforce planning, and spare parts management. After a review of several available products, KJCOC selected the MPAC software product (Maintenance Planning and Control, vintage 1993, Systems Works, Inc.) to perform the functions listed above (see Appendix K). While this powerful tool greatly improved the efficiency of O\&M management, the older version implemented by KJCOC was not as user friendly (i.e., not Windows-based) as current versions of MPAC or other competing CMMS software, and was too costly to purchase and maintain relative to a CMMS that KJCOC would recommend today. Consequently, KJCOC may replace MPAC with an alternate CMMS product in the near future.

None of the CMMS products reviewed by KJCOC afforded adequate maintenance management of the large solar fields (total collector surface is approximately $1 \mathrm{~km}^{2}$ ), which consist of thousands of components of the same type. KJCOC therefore developed specialized software,

\footnotetext{
${ }^{2}$ LUZ International, Ltd. devised a larger power park at Harper Lake, California, consisting of up to six 80-MW plants with a common control room, raw water treatment, and electrical switch gear. Two of these plants were implemented but the rest were never built.
} 


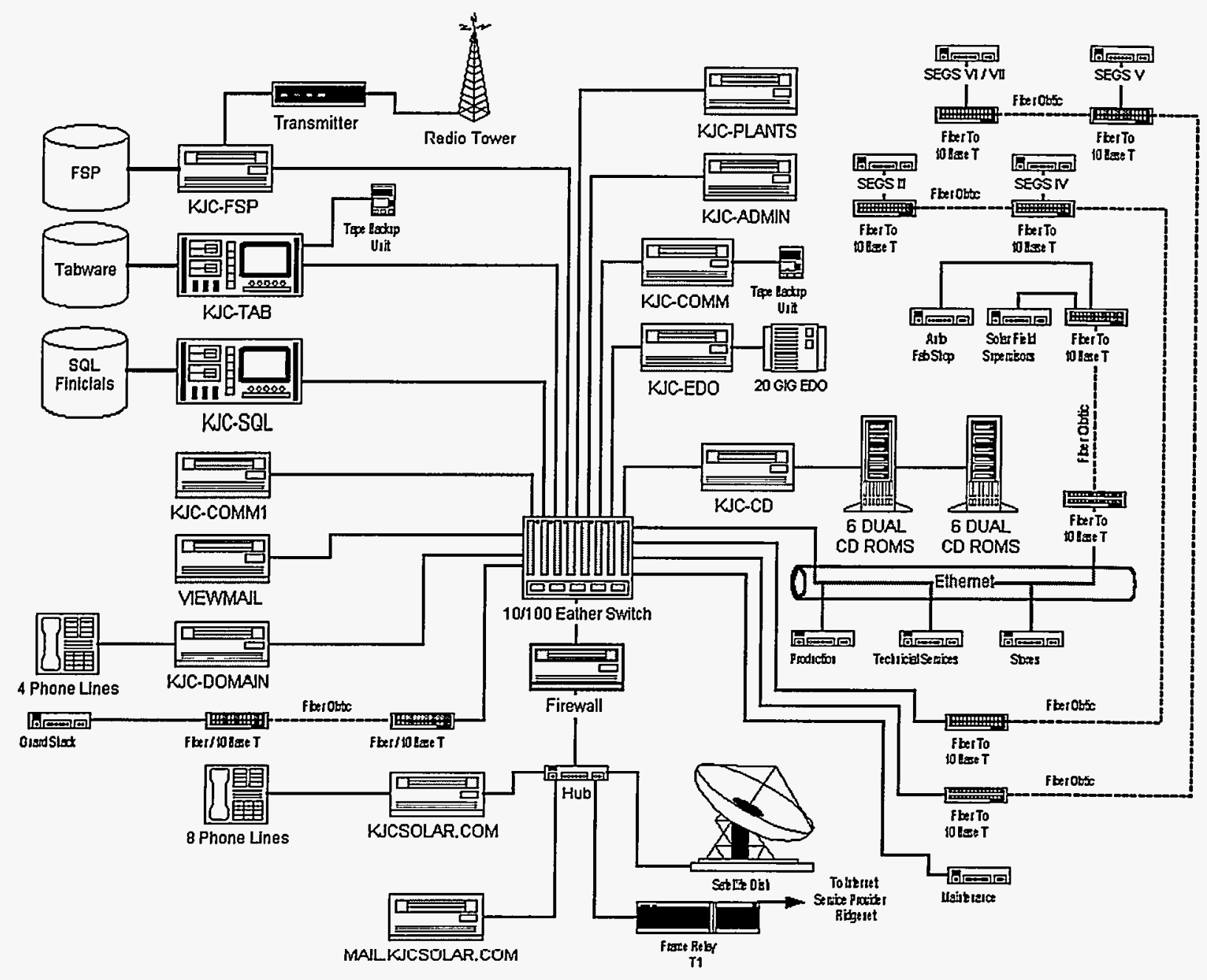

Figure 11. Fiber-optic network at Kramer Junction.

known as FSP (Field Status Program), to manage the solar fields (see Appendix L). As shown in Figure 12, data are entered through a user-friendly interface that graphically shows the status of each subcomponent within an SCA. The FSP was interfaced with the MPAC software to facilitate the generation of work orders to fix or replace failed components in the solar field. Furthermore, KJCOC installed a radio network that allows technicians working in the field to remotely enter FSP data; the user interface is a computer screen that is attached to the windshield visors of the solar-field trucks (see Appendix M). The radio system is shown in Figure 13. The FSP software has become an integral part of the solar field maintenance management by KJCOC, and similar software is recommended for future projects. The radio-data-entry feature is also recommended for projects with very large solar fields, like those at Kramer Junction.

Before the O\&MIP, only paper-based maintenance procedures and plant drawings were available to the power park maintenance crew. A systematic review of the teardown and rebuild procedures for various components found them lacking in detail, and it was decided that the content of most procedures should be greatly expanded. During the rewrite, it was decided that electronic versions of the procedures and associated piping and instrument drawings should be placed on the network to facilitate future updates to these documents and to allow maintenance crew anywhere within the power park to download copies for their use (see Appendix N). The O\&MIP 
provided only a small amount of funding to test this new method of managing maintenance documents. KJCOC was very pleased with the results and used its own funds to develop many more electronic documents than were funded by the O\&MIP (by a factor of close to 10).

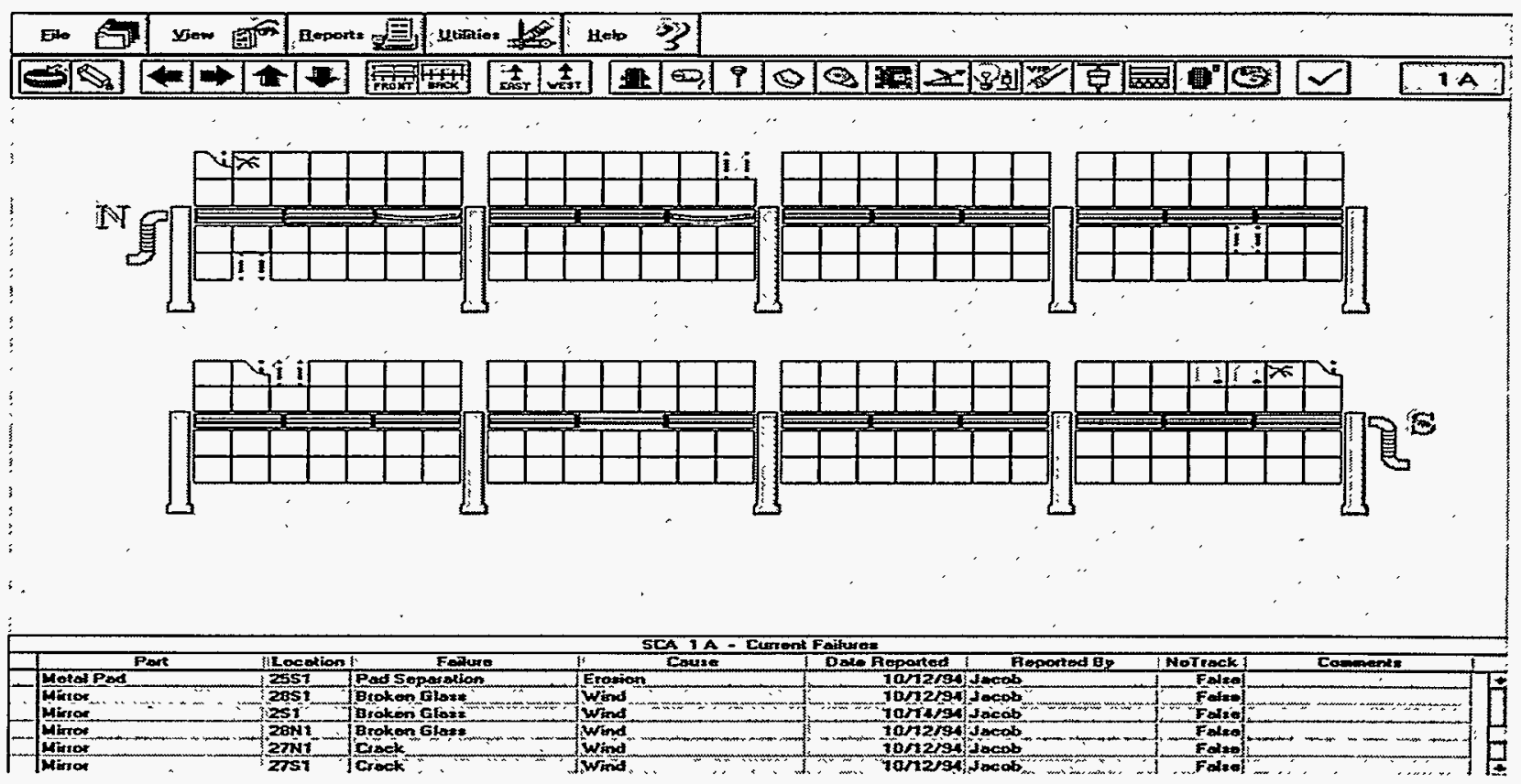

Figure 12. Graphic-user interface for the Field Status Program.

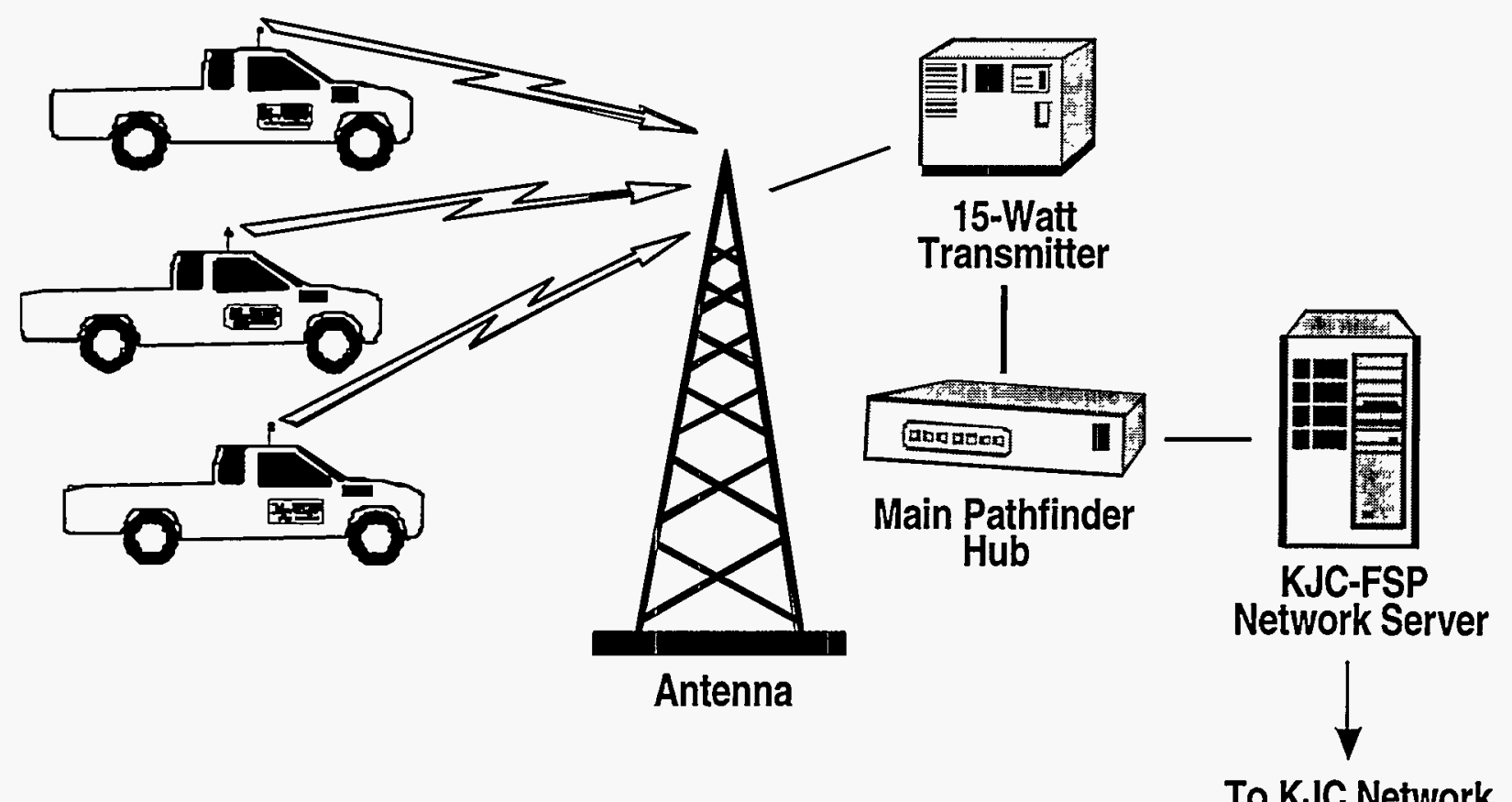

Figure 13. Maintenance data are entered from the solar field via radio transmitters. 


\section{Operations}

Traditionally at Kramer Junction and other power plants, control-room operators have manually recorded operations data into logbooks and databases. Operations data include such items as turbine on-line and off-line times; a list of equipment that is out of service; operation events; and gas, electricity, and water consumption. Besides acting as a log for legal purposes, performanceengineering staff review this data to help them with studies aimed at improving reliability and increasing the performance of the plant. Because the old system at Kramer Junction was largely paper based, data retrieval was inefficient, which encumbered detailed understanding of the factors that impact plant reliability and performance. Consequently, a much-improved "computer-based operator log" was developed as part of the O\&MIP that allowed easy access and analysis of historical operations data via the site network (see Appendix O). The software has also proven to be very useful in preparing monthly summary reports that are provided to the plant owners. One of the data entry modules from this OpLog@ software is shown in Figure 14. Use of the OpLog@ system is now an integral part of the daily activities at Kramer Junction.

In addition to providing easy access to O\&M databases, interconnection of all control rooms via the site network has also allowed the exciting possibility of controlling all five power plants from a remote location such as the administration building. If successful, the number of operators on site might be reduced by a factor of three to five. However, because of the perceived risk, interconnection was not attempted while the plants were on line during the O\&MIP. Rather, it was demonstrated that the operators at one SEGS unit could monitor the status and control

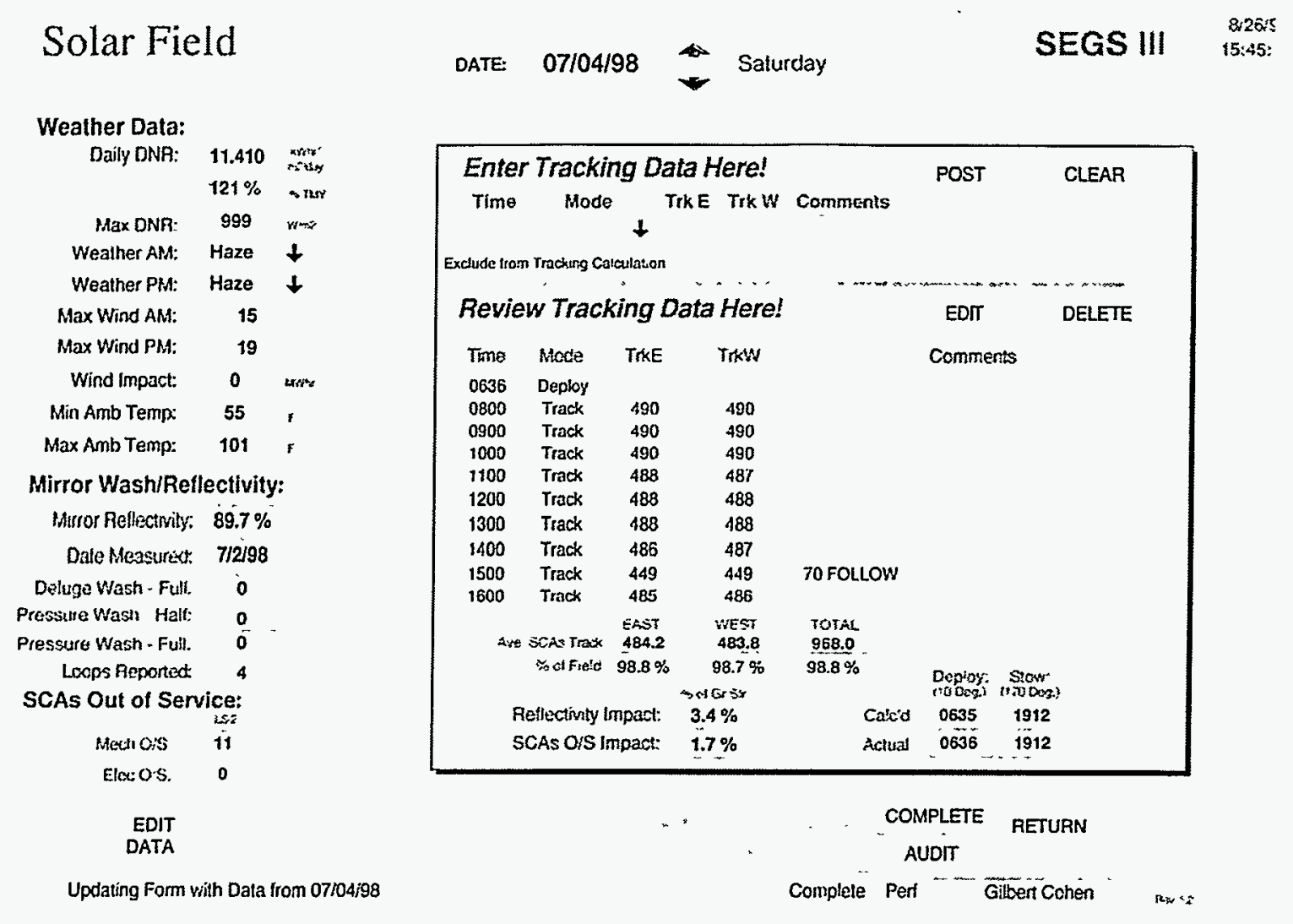

Figure 14. A data entry sample of a computerized operator log. 
another SEGS unit during the nightly shutdown period. This remote control will eventually allow a significant reduction in the number of nighttime operators required at the Kramer Junction power park.

\section{Improving the Reliability of Solar Field Flow-Loop Hardware}

Solar energy is collected and transferred to the electric power generation system by circulating synthetic oil heat transfer fluid (HTF) between the collector field and the steam generator. Before the O\&MIP, significant reliability problems were an issue with several of the components within the HTF flow loop. These problems led to unnecessary plant outages and high costs because of frequent maintenance and replacement of worn-out parts.

\section{Pumps}

In the early years of operation at Kramer Junction, the pumps that circulate the HTF to the solar field experienced many seal failures at SEGS III through V and were a significant contributor to maintenance costs. For example, the seal for one pump at SEGS $V$ failed 42 times within 12 months at a materials and labor cost of $\$ 250,000$, and when accounting for the loss of electricity production, the cost was even higher. In an attempt to remedy the problem, the O\&MIP first installed a graphite-fiber seal that proved unsuccessful. Next we tried a double-bellows-type mechanical seal, offering expectations of better sealing and increased durability. In addition to the installation of the new seal, a heat exchanger was added to the pump coolant loop to maintain the seal at a lower operating temperature, and the pump suction pressure was raised to increase the net positive suction head to the pump. Finally, the pressure was rebalanced between this pump and another operating in parallel. As a result of all these changes, the pump ran flawlessly for a period of one year during 1993. After that time, other similar pumps at Kramer Junction were retrofitted with the same fix (see Appendix P).

\section{Flexible Connections}

Each of the approximately 1,000 SCAs within a 30-MW solar field can be rotated independently of adjacent SCAs. This independent rotation allows one SCA to track the sun while the adjacent one is rotated off sun to perform maintenance, such as control system repair. Thus, the flow-loop connection between SCAs must be flexible. When the SEGS plants were built, flexible hoses were used to establish the connection. They are made up of a corrugated steel inner tube that is enclosed in stainless steel mesh, covered with insulation, and further enclosed in an outer corrugated tube. Before the O\&MIP, reliability of the flex hoses was marginal, and on occasion, catastrophic failure would lead to HTF spills and fires. It was also known that pressure drops were high because of the inner corrugations within the hose.

In an attempt to reduce the problems associated with flex hoses, KJCOC worked first with BARCO USA and finally with Advanced Thermal Systems to develop a rotating-ball-joint-type 
connection suitable for application within a solar field. These joints offered the potential of higher reliability, lower investment costs, and lower parasitic power requirements because of reduced pressure loss (Figure 15 shows ball joints and flex hoses).
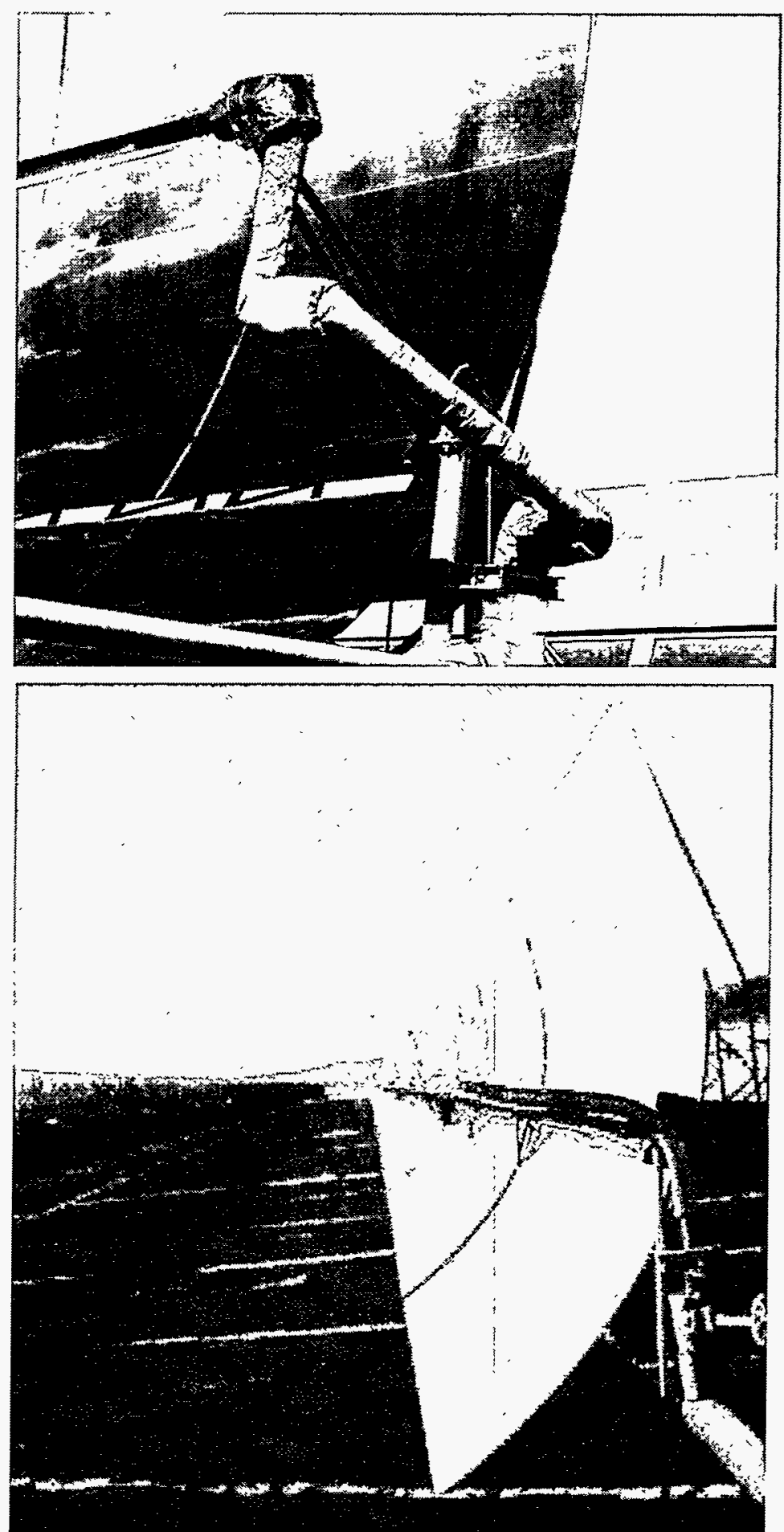

Figure 15. Ball joints (top) rather than flex hoses (bottom) are now the preferred method of interconnecting SCAs. 
The O\&MIP installed special test rigs in order to subject them to 30 years of cycles. Pressure loss measurements verified that pressure drop within a loop of eight SCAs was $45 \%$ less for rotating joints than for flex hoses. After extensive testing, only minor vapor emissions from the joints were noted and no catastrophic failures occurred. ${ }^{3}$ Vapor emissions can be easily corrected by repacking the joint. These tests have led to a high confidence in the use of ball joints and hundreds of them have now been installed at Kramer Junction and Harper Lake SEGS plants (see Appendix Q). Future solar trough projects will also use ball joints instead of flex hoses.

\section{Flow Meters}

To run the plant at maximum efficiency, control-room operators and plant-performance engineers need to have an accurate knowledge of HTF flow rates through the solar fields. The reliability of the flow meters is also important because failures could lead to plant outages. In addition, the meter must be easily maintained to keep maintenance cost to a minimum. Before the O\&MIP, four types of flow meters were used at Kramer Junction with varying degrees of success: annubar, insertion turbine, orifice, and low-loss flow nozzles. Each type of flow meter had advantages over the others, but none had proven to be sufficiently accurate and/or reliable.

During the O\&MIP, new flow meters based on vortex-shedding and acoustic measurement methods were extensively tested and compared with the previous baseline set of meters (see Appendix R). Initial results suggested the vortex method was preferred because it was more accurate than the baseline methods and the acoustic method. However, from a maintainability point of view, the acoustic meter was highly desirable because it was easily attached to the outside of the pipe and was readily accessible. KJCOC continued to work with the acoustic manufacturer (Panametrics Inc.) to improve the accuracy of the meter. After a few years of trial and error, an acceptable acoustic meter was finally developed. KJCOC subsequently replaced all prime HTF flow meters at the five Kramer Junction plants with the new acoustic flow meters.

\section{Developing an Optimum Plant Operating Strategy}

How to best operate a solar plant during ever-changing weather conditions and plant equipment states is a challenging problem. In this section, we will discuss methods and technology the O\&MIP developed to help the O\&M crew and plant engineers decide how to best operate the plant to achieve maximum electricity output.

\section{Weather Prediction}

Accurate foreknowledge of the weather would be of great value to both operations and maintenance. With respect to maintenance, a few days' warning on weather can permit the planning of mini-shutdowns during a weekend or weekdays when poor weather would otherwise limit solar production, or it can prevent taking a plant off-line for maintenance when solar conditions are good. With respect to operations, the hybrid gas-use strategy would be better defined. For

\footnotetext{
${ }^{3}$ A severe failure of a ball-joint connection recently occurred at the Harper Lake SEGS plants. Improper mounting was determined to be the root cause of the failure, suggesting that extra care must be taken during installation.
} 
example, if several days of bad weather are forecast, the fossil-boiler would be prepared for operation.

Historically, KJCOC operators have relied on "look-at-the-sky" observations and normal weather forecasts to make weather decisions. In an attempt to make a significant improvement over this baseline approach, the O\&MIP hired the services of a scientist who specializes in the prediction of insolation and weather. A three-month trial was conducted in which the scientist provided hourly predictions of direct-normal insolation, one and two days in advance. While the predictions proved to be more accurate than historical methods, the cost of the service was high ( $\$ 100,000$ per year) and was hard to justify. KJCOC ultimately decided to use weather predictions from the DTN Weather Center and the Internet. While these services do not predict insolation levels, operators can view area-specific satellite maps, current weather conditions, and shortand long-range forecasts. These services cost less than $\$ 1,000$ per year and have proven to be useful in developing weather-influenced O\&M strategies (see Appendix S).

\section{High-Wind Operating Strategy}

Solar field operation becomes quite challenging during times of high wind velocities at Kramer Junction. Mirrors can be broken by wind forces and subsequently initiate a limited cascade effect, breaking glass envelopes of the HCEs and/or more mirrors. To protect the plant, the operators rotate the mirrors in the field to a wind-safe position when winds exceed 35 miles per hour. Plant data examined at the beginning of the O\&MIP suggested that energy losses caused by high-wind stow of the fields were significant and was about $40 \%$ as important as the degraded HCE problem described previously. Longer operation hours without a total stow of the collector field may be possible if a high-wind operating strategy could be devised that simultaneously protected the field from damage and allowed most of the field to continue to operate.

The key to developing an optimized high-wind operating strategy was a thorough understanding of the wind loads on the collectors. Special load-cell instrumentation was applied to a collector in the windward area of the solar field and a series of experiments evaluated 10 different windprotection schemes (see Appendix T). The scheme that appeared to work best was to stow every fourth row of collectors. However, because of several uncertainties regarding wind forces and wind patterns, the chosen strategy must still incorporate a significant margin of conservatism.

Besides stowing the minimum number of collectors during high winds, another way to improve the performance of the solar field is to reduce the HTF flow in rows that are stowed. This would reduce the mixing of large quantities of cold HTF emanating from stowed rows with hot HTF in rows that are tracking the sun. In the current design, the operators cannot change the flow in a particular row. To allow this possibility, remotely controlled motor-operated valves (MOVs) need to be installed in the rows that are to be stowed. To test this and other ideas regarding more precise flow control, the O\&MIP installed remotely controlled MOVs and automatic flow-loop control on two loops at SEGS V. Tests conducted at the end of the O\&MIP demonstrated a clear performance benefit of automatic control over manual control during windy weather and other operating conditions (see Appendix U). However, full implementation of automatic control adds design complexity (i.e., 50 MOVs and associated controls per 30-MW SEGS) and an unknown effect on O\&M costs. Because of lack of long-term O\&M cost data, we are unable to recommend whether it is prudent to implement automatic flow control in future trough plants. 


\section{Plant Performance Modeling}

The O\&MIP developed two different types of analytical models to gain a better understanding of the factors that influence plant performance. Using actual insolation and weather data collected on site, the models predict what the electric and thermal performance of the plants should be. Both plant models contain subroutines for each of the plant subsystems that are analytically and/or empirically derived. For example, the thermal performance of the collector field is based on validated models (see Appendix V) developed during the testing of the LS-2 collector on the SNL rotating platform, described previously. Intelligent use of the models allows plant engineering and the operators to identify systems that are operating below design levels, and consequently to plan corrective measures.

In the first model, predictions of performance are compared with the actuals on an hourly basis. This model allows the engineering staff to identify loss mechanisms that in turn are used to prioritize corrective O\&M activities (see Appendix W). Because the SEGS plants are hybrid, the model is also used to develop the proper gas-use strategy to maximize electricity-sales revenues. This hourly model is typically exercised by the engineering staff once per month. Results from this analysis constitute a major portion of the monthly performance report provided to the site managers and plant owners.

Use of the hourly performance model suffers from the fact that results are not generated in real time, i.e., the operators and engineers cannot study the performance of the plant at a given time on a given day. Thus, the O\&MIP worked with Black \& Veatch to develop a second model called the On-line Performance Monitoring (OPM) system (see Appendix X). This model automatically and continuously updates to reflect the actual state of the plant equipment and operates on a separate computer terminal within the SEGS VI and VII control room (see Figure 16).

The predictive "what if" capability of the OPM allows the operators and plant engineers to determine the probable results of proposed changes in key operating parameters. For example, the operator may want to understand how plant electricity output will change if the solar field operating temperature is lowered. The proposed operating temperature change is input and the OPM system will show the results of the actions. At the date of this writing, the control-room operators are not fully conversant with OPM and it has only been used on a limited basis by the engineering staff. Thus, we are unable to report on the usefulness of this tool in day-to-day operation.

The accuracy of each of the two models is only as good as the weather data feeding it. Thus, to ensure accurate weather data, the O\&MIP developed a quality-assured centralized weather station to measure insolation, wind, and temperature (see Appendix Y). The key to achieving quality data was the implementation of the recently developed Data Quality Management System software, shown in Figure 17. Before the O\&MIP, each of the five SEGS plants relied on their own weather station, which often suffered from data quality problems. 


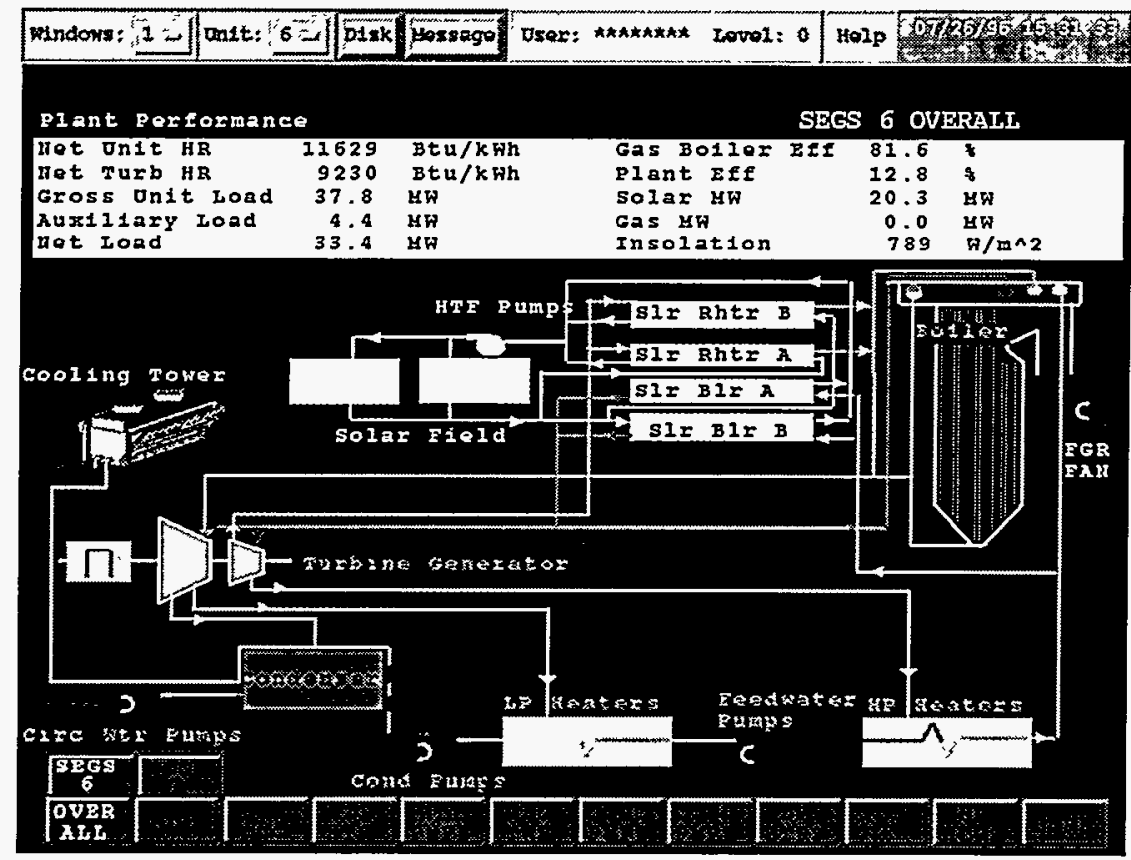

Figure 16. This OPM view screen operates continuously in the SEGS VI control room.

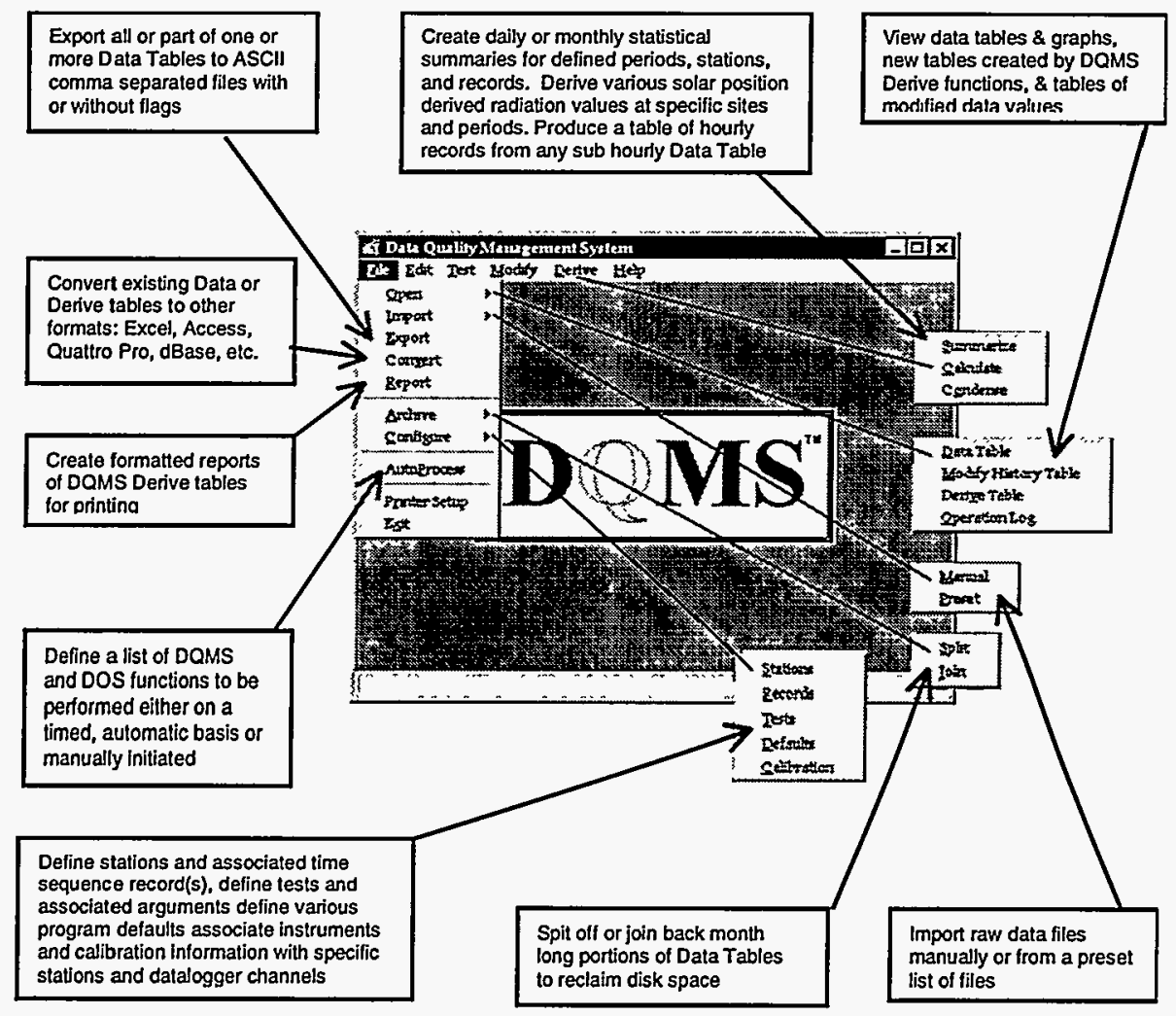

Figure 17. The Data Quality Management System, commercially available from Augustyn + Co., processes the daily data using qualitycontrol methods developed by the National Renewable Energy Laboratory. 


\section{Reducing Costs Associated with Environmental Issues}

Concentrating solar power plants are an environmentally friendly technology. To maintain that apt image, it is important they have a minimal impact on the local desert surroundings. Air quality and water use are significant environmental issues that are also important to O\&M costs. However, as will be shown in this section, reducing environmental impact can actually lead to lower O\&M costs, a win-win situation that does not happen often in real life.

\section{Volatile Organic Compounds Emissions and Oil Spills}

Each 30-MW solar field contains approximately 100,000 gallons of synthetic heat transfer oil. During operation of the solar field loop and during the shutdown period each evening, a small portion of oil is lost through volatilization. These "fugitive emissions" are classified as volatile organic compounds (VOCs), and the Environmental Protection Agency (EPA) is concerned with them. KJCOC is also interested in reducing losses because this fluid is relatively expensive (\$13 per gallon). Consequently, the O\&MIP identified and quantified heat transfer oil losses at each of the five plants (see Appendix Z). This work showed that in the early days of the program, up to $1 \%$ of the total inventory could be lost in a year. However, most of the loss was from oil spills rather than through volatilization $(0.08 \%)$. Improved HTF handling methods, better O\&M on flexible hoses, and the extensive use of replacement ball joint assemblies have greatly reduced inventory loss so that currently the total plant loss is less than $0.5 \%$ per year. When spills that contaminate soil do occur, the soil is rendered harmless through use of on-site bioremediation methods that KJCOC developed outside the O\&MIP. Volatile emissions from the solar loops were found to be much lower than estimates based on EPA emission factors. Thus, O\&M crews at trough plants should devote much more attention trying to prevent and mitigate spills rather than worrying about VOC emissions.

\section{Water Use}

Water is not plentiful in desert environments and is therefore a relatively expensive commodity. This is certainly true at Kramer Junction, where the cost of raw water and water-treatment chemicals is a significant portion of the annual O\&M budget (approximately $\$ 1$ million per year). To reduce consumption, the O\&MIP installed water instrumentation and performed a study to assess water use and quality at the power park (see Appendix AA). Results of the study indicated only $1.4 \%$ of the total water is used for mirror washing, and more than $90 \%$ is associated with operation of the Rankine power-cycle equipment. The assessment of water quality suggested that blowdown of the Rankine cycle equipment was occurring too frequently, leading to higher-than-needed water use. This greatly improved understanding of water use and quality led to water-conservation measures that resulted in a 33\% reduction in water use per megawatt hour of electricity produced, as indicated in Figure 18. 


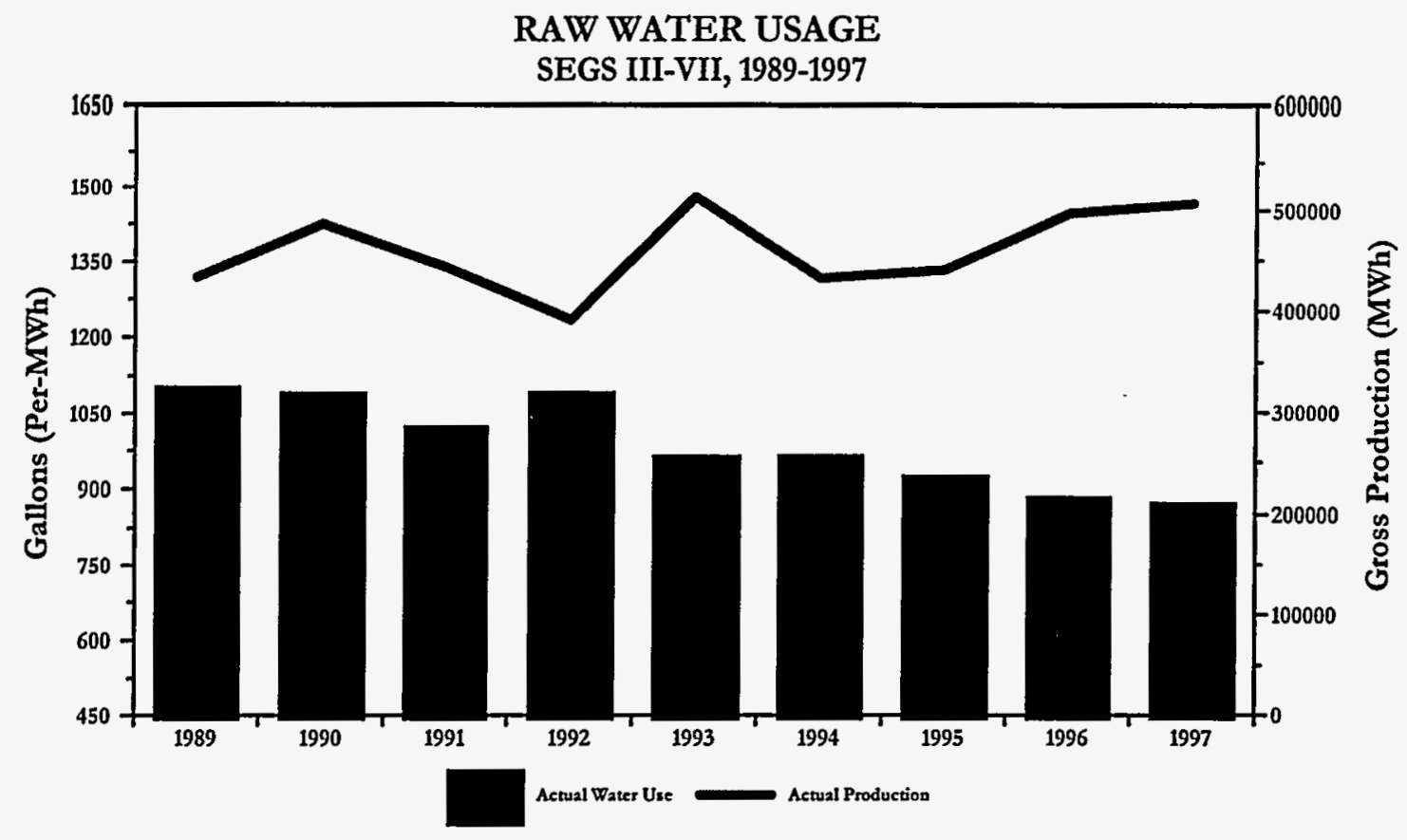

Figure 18. Raw water usage versus gross electricity produced for SEGS III through VII.

\section{Developing an Optimum O\&M Plan}

The recommended O\&M plan for the solar field (see Appendix $A B$ ) is based on the experience and knowledge gained at Kramer Junction but differs somewhat from the exact staffing structure at that site. Rather, this plan is oriented toward a new facility that would fully benefit in the design and construction phases from the lessons learned at the SEGS plants. It also attempts to accommodate varying O\&M staffing philosophies by formulating staffing from nominal and reduced staffing perspectives. It is very difficult, if not impossible, to relate specific O\&M structures and approaches to long-term O\&M costs. For example, an O\&M group with full predictive and preventive O\&M programs may save equipment costs in the end but is characterized by higher near-term labor costs. In an attempt to accommodate different organizational perspectives, nominal values reflect what we believe is the staffing plan that provides the most cost-effective trade-off between labor costs and equipment reliability. The reduced staffing, on the other hand, represents the minimum staffing level that we believe to be prudent, though "bare bones," for adequate solar field maintenance.

Several plausible O\&M scenarios are proposed for developed and developing countries that include the costs for staffing, materials, and specialized equipment. Estimates are given only for the solar field portion of the plant because numerous O\&M estimates are already available in the literature for the power block, i.e., Rankine steam cycle or gas-turbine combined cycle. Depending on the specific circumstance, an incremental staff of between 13 and 31 people would be necessary to maintain the solar-specific portion of a plant about one-half the size of Kramer Junction (approximately $500,000 \mathrm{~m}^{2}$ ). Combining labor costs with materials and special equipment costs results in an annual $O \& M$ cost of between $0.8 \notin$ and $1.2 \phi / \mathrm{kWh}$, depending on the specific O\&M scenario. For larger plants, O\&M costs are projected to drop as low as $0.5 \notin / \mathrm{kWh}$. 


\section{Comparing O\&M Costs Before and After the O\&MIP}

Over the course of the O\&MIP, the total O\&M costs at Kramer Junction have reduced significantly, and overall plant output has increased dramatically. Figure 19 shows these gains, which illustrate a 37\% reduction in annual O\&M costs and a $31 \%$ increase in annual gross electrical output. Costs include on-site and contractor labor, spare parts, and consumables for the entire site, but exclude natural gas purchases. Costs do not include items categorized as major maintenance. These items correct construction deficiencies or newly planned modifications and are not considered standard O\&M. Plant output includes a 25\% annual heat input to the power block from the natural gas boilers.

Several other factors besides the O\&MIP have had major impacts on these achievements, including the parallel growth in effectiveness of the O\&M organization, major power block maintenance programs approved by the owners, and the replacement of some critical spare parts whose supply was interrupted by the demise of LUZ. In addition, while the O\&MIP provided the engineering and prototype implementation of many improvements, it was an owner decision to separately fund plant-wide implementations. The O\&M group became more streamlined over this period, and training programs were aggressively pursued in parallel with the improvements in O\&M practices that have been described above. Preventive and predictive maintenance programs were initiated and carried out, reducing reactive maintenance costs and greatly increasing cost-effectiveness. By any measure, however, the gains embodied in these two yardsticks are an impressive demonstration of both the benefits of the O\&MIP and the work done by KJCOC.

\section{O\&M Cost Reduction Program}

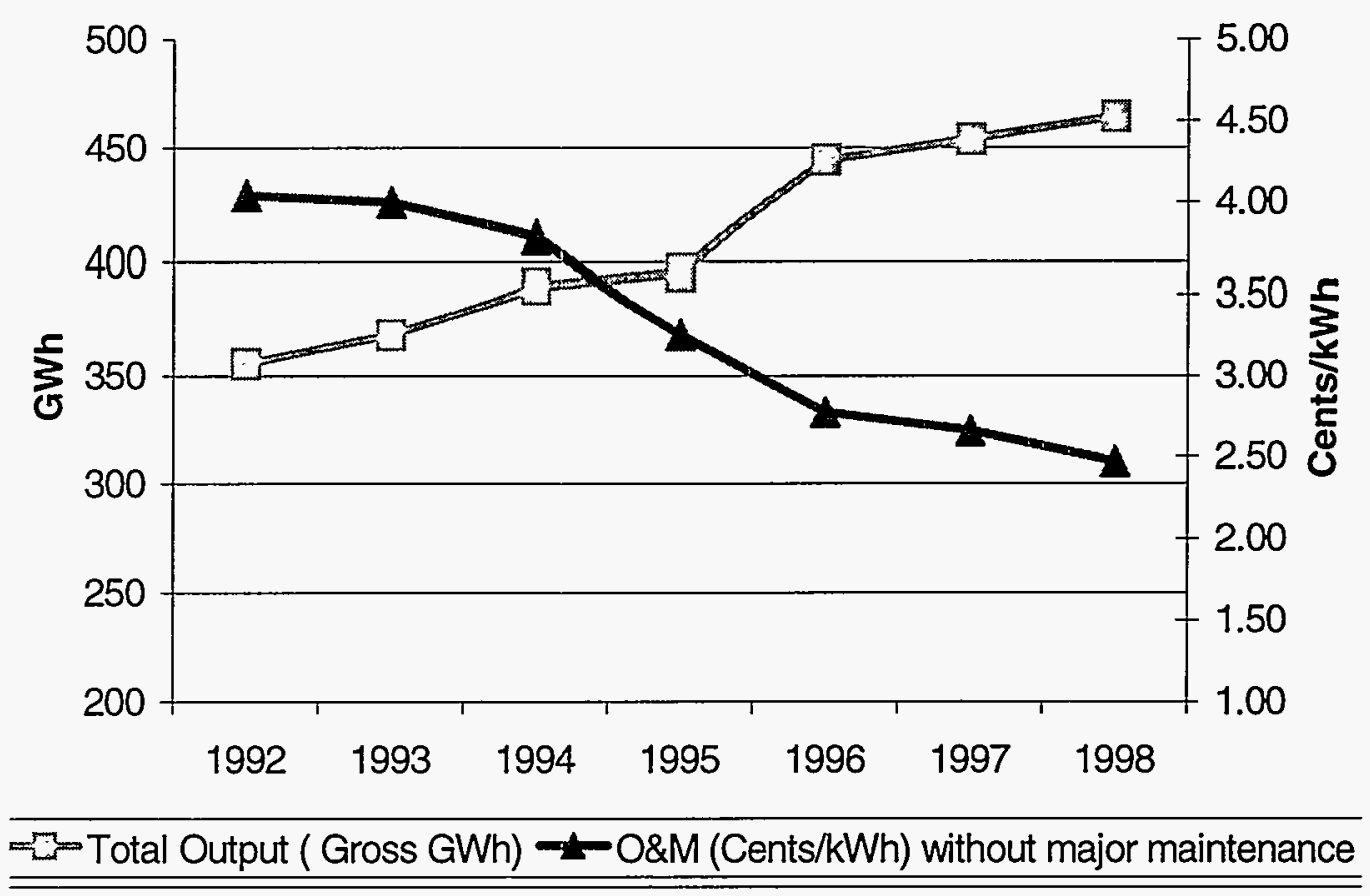

Figure 19. O\&M costs and electrical output at Kramer Junction during the years 1992 to 1998. 


\section{Bibliography}

Elements of this program have been presented in a series of conference papers and talks since the inception of the work. These papers and presentations are listed below in chronological order, and have been reproduced in a project document that is available upon request ${ }^{4}$ from Greg Kolb at Sandia National Laboratories.

G. Cohen, R. Cable, H. Price, R. Mahoney, and V. Dudley, Efficiency Testing of SEGS Parabolic Trough Collector, Proceedings of the 1993 Annual Conference of the American Solar Energy Society (Solar 93), Washington, D.C., April 1993.

D. Kearney, O\&M Cost Reduction for Solar Thermal Electric Plants, 1993 Annual Conference of the Solar Energy Industries Association (Soltech 93), Washington, D.C., April 1993.

V. Dudley, et al., Test Results: SEGS LS-2 Solar Collector, SAND94-1884, Sandia National Laboratories, Albuquerque, New Mexico, 1994.

G. Cohen, D. Kearney, and G. Kolb, Comparison of SEGS Solar Field Performance from Laboratory and Operating Plant Testing, Proceedings of the SOLCOM I Conference, Jerusalem, Israel, February 1994.

G. Cohen, Concepts for Technology Advancements and Plant Configurations, 1994 Annual Conference of the Solar Energy Industries Association (Soltech 94), Ponte Vedra Beach, Florida, March 1994.

D. Kearney, Current Results of KJC OC/Sandia O\&M Cost Reduction Program, 1994 Annual Conference of the Solar Energy Industries Association (Soltech 94), Ponte Vedra Beach, Florida, March 1994.

G. Cohen and D. Kearney, Improved Parabolic Trough Solar Electric Systems Based on the SEGS Experience, Proceedings of the 1994 Annual Conference of the American Solar Energy Society (Solar 94), San Jose, California, June 1994.

S. Frier and G. Cohen, O\&M Planning and Implementation for Solar Thermal Electric Plants, Proceedings of the 1994 Annual Conference of the American Solar Energy Society (Solar 94), San Jose, California, June 1994.

G. Cohen, Achievements in Solar Parabolic Trough Technology, Proceedings of the 7th International Symposium on Solar Thermal Concentrating Technologies, Moscow, Russia, September 1994.

G. Cohen, Hybrid Operation in SEGS Plants, Hybrid Solar Thermal Electric Workshop, National Renewal Energy Laboratory, Golden, Colorado, November 1994.

\footnotetext{
${ }^{4}$ O\&M Cost Reduction in Solar Thermal Electric Plants: Compendium of Conference Presentations, Sandia National Laboratories Contract No. AB-0227, KJC Operating Company, 1993-1997.
} 
F. Lippke, Simulation of the Part-Load Behavior of a 30-MW SEGS Plant, SAND95-1293, Sandia National Laboratories, Albuquerque, New Mexico, 1995.

S. Frier, SEGS Operational Experience, Proceedings of the Workshop on Solar Thermal Trough Power Plant Technology and its Transferability to the Mediterranean Region, Sevilla, Spain, January 1995.

G. Kolb, Evaluation of Power Production from the Solar Electric Generating Systems at Kramer Junction: 1988 to 1993, Proceedings of the ASME/JSME/JSES International Solar Energy Conference, Maui, Hawaii, March 1995.

H. Price, P. Svoboda, and D. Kearney, Validation of the FLAGSOL Parabolic Trough Solar Power Plant Performance Model, Proceedings of the ASME/JSME/JSES International Solar Energy Conference, Maui, Hawaii, March 1995.

G. Cohen, The Solar Industry Perspective, Optical Materials Industry Advisory Panel, National Renewal Energy Laboratory, Golden, Colorado, June 1995.

G. Cohen, D. Kearney, and G. Kolb, Recent Accomplishments on Cost Reductions in O\&M for Solar Thermal Electric Plants, Proceedings of the 1995 Annual Conference of the American Solar Energy Society (Solar 95), Minneapolis, Minnesota, July 1995.

D. Rib, Environmental Aspects of SEGS Solar Power Plants, Proceedings of the 1995 Annual Conference of the American Solar Energy Society (Solar 95), Minneapolis, Minnesota, July 1995.

G. Cohen, O\&M Cost Reduction, IEA SolarPACES-Task I: Electric Power Systems Meeting, Stuttgart, Germany, October 1995.

G. Cohen, D. Kearney, and R. Cable, Recent Improvements and Performance Experience at the Kramer Junction SEGS Plants, Verein Deutscher Ingenieure (VDI) Conference on Solar Thermal Technology, Stuttgart, Germany, October 1995.

R. Cable, G. Cohen, and D. Kearney, Experience with Mirror Reflector Soiling and Washing Methods at the Kramer Junction SEGS Plants, Proceedings of the 1996 Annual Conference of the American Solar Energy Society (Solar 96), Asheville, North Carolina, April 1996.

G. Cohen, D. Kearney, and R. Cable, Recent Improvements and Performance Experience at the Kramer Junction SEGS Plants, ASME Solar Energy Division Meeting, San Antonio, Texas, April 1996.

T. Jones, N. Craig, J. K. Shouse, R. Leonard, and R. Cable, On-Line Performance Monitoring of SEGS VI and VII, ASME Solar Energy Division Meeting, San Antonio, Texas, April 1996.

F. Lippke, Operating Strategy and Its Impact on the Performance of a 30-MW $W_{e}$ SEGS Plant, ASME Solar Energy Division Meeting, San Antonio, Texas, April 1996.

G. Cohen, O\&M Cost Reduction Program, Solar Thermal Technology Power Program SemiAnnual Review, National Solar Thermal Test Facility, Albuquerque, New Mexico, June 1996. 
N. Amarnani, R. Cable, and G. Cohen, Optimized Solar Field Operation During High Wind Velocities at the Kramer Junction SEGS by Determining Transient Forces, Solar Energy Forum (ASME session), Washington, D.C., April 1997.

G. Cohen and S. Frier, Ten Years of Solar Power Plant Operation in the Mojave Desert, Solar Energy Forum (ASES session), Washington, D.C., April 1997.

G. Cohen, $O \& M$ Cost Reduction, IEA/SolarPACES Working Group Meeting, Albuquerque, New Mexico, September 1997.

G. Cohen, D. Kearney, and G. Kolb, Overview of the Kramer Junction/Sandia O\&M Cost Reduction Program for Solar Thermal Electric Plants, Proceedings of the 9th International Symposium on Solar Thermal Concentrating Technologies, Font Romeu, France, June 21-26, 1998.

R. J. Nelson and R. G. Cable, The KJC Plant Performance Model - An Improved SEGS Plant Simulation, Proceedings of the ASME/JSME/JSES/KSME International Conference, Maui, Hawaii, April 11-14, 1999. 
This page intentionally left blank. 


\section{Appendix A \\ TESTING OF THE LS-2 TROUGH COLLECTOR AT SANDIA NATIONAL LABORATORIES ${ }^{1}$}

In this appendix we present (1) optical property measurements taken on a number of the original heat collection element (HCE) tubes manufactured by LUZ International Ltd. with black chrome or cermet selective surfaces and (2) thermal efficiency measurements of a segment of an LS-2 collector that was tested on the Sandia National Laboratories (SNL) rotating platform, given a variety of $\mathrm{HCE}$ conditions.

\section{Background}

The HCEs consist of 70-mm diameter steel tubes, four meters in length, with a black chrome or cermet selective surface, surrounded by an evacuated glass tube. A glass-to-metal seal and a bellows at each end of the tube provide the vacuum-tight enclosure while also allowing for differential thermal expansion between the glass and metal tube. The vacuum enclosure serves to protect the selective surface and to reduce convective heat losses, and the selective surface helps improve the absorption of solar radiation while minimizing radiation heat losses. The LS-2 HCEs used a black chrome selective surface, developed by LUZ International Ltd., which could be used at temperatures up to $350^{\circ} \mathrm{C}$. However, later solar electric generating system (SEGS) plants required outlet temperatures of up to $400^{\circ} \mathrm{C}$ to achieve higher power cycle efficiencies. Thus, LUZ developed the cermet (graded ceramic/metal applied by a sputtering process) selective surface coating that could be operated at temperatures much higher than the black chrome. In addition, the cermet surface has reduced radiation losses because of a lower emittance and does not oxidize or otherwise degrade if air enters the annulus. The surrounding glass tube has an anti-reflective coating on both surfaces. Getters (metallic substances that are designed to absorb gas molecules) and special hydrogen removal devices are installed in the vacuum space to absorb hydrogen and other gases that migrate into the vacuum annulus over time.

The condition of the HCEs has a major impact on collector thermal performance. The HCEs can lose vacuum because of hydrogen migration from the heat transfer fluid through the steel receiver tube into the vacuum annulus, or as a result of a crack or leak in the glass or bellows that allows air into the vacuum annulus. There are numerous causes of HCE glass breakage. The glass can break because of poor alignment during installation, operational incidents, or physical interference from other components such as a mirror breaking in the wind and falling on the HCE.

\section{HCE Selective Surface Property Testing}

The optical and thermal characteristics of the HCE play a significant role in the overall efficiency of the LUZ collectors. Therefore, a good understanding of the actual HCE selective surface properties is needed to fully understand the performance of the solar fields. Twelve

\footnotetext{
1 This paper is an excerpt from V. Dudley, G. Cohen, R. Cable, H. Price, and R. Mahoney, Efficiency Testing of SEGS Parabolic Trough Collector, Proceedings of the 1993 American Solar Energy Society Annual Conference, Washington, D.C., April 22-28, 1993.
} 
HCE samples (four cermet, eight black chrome) were sent to SNL to test the selective surface absorptance and emittance. The samples included both new HCEs and HCEs removed from the plants. The HCE samples included new and used HCEs with good vacuum, used HCEs that had lost vacuum (i.e., air was present between the glass envelope and receiver tube), and used HCEs with the glass broken. SNL used the ASTM E903-82 procedure for testing the HCE samples. ${ }^{2}$

Figure A-1 shows the results of the HCE property test measurements performed by SNL. The figure shows the normal emittance plotted against the solar absorptance for each sample tested. The emittance of the black chrome samples are shown at $300^{\circ} \mathrm{C}$ and the cermet samples at $350^{\circ} \mathrm{C}$. These temperatures correspond to the average operating temperatures in which these surfaces operate. Also shown are the design points used in the original performance projections.

The goal is to achieve an absorber surface with low emittance and high solar absorptance, with the two properties optimized for the desired operating temperature range and absorber design. Although solar absorptance does not vary temperature, emittance is temperature dependent. Figure A-2 shows the emittance of the black chrome and cermet selective surfaces. Note that the emittance of black chrome can be calculated for temperatures above its maximum operating temperature of $350^{\circ} \mathrm{C}$.

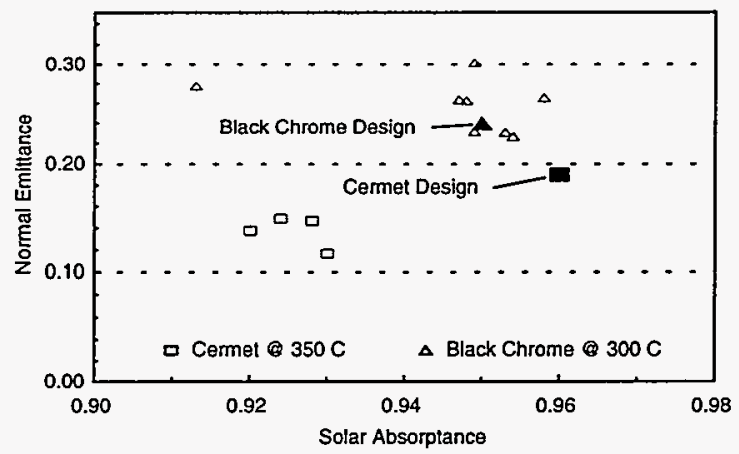

Figure A-1. Black chrome and cermet HCE property test results (emittance versus solar absorptance).

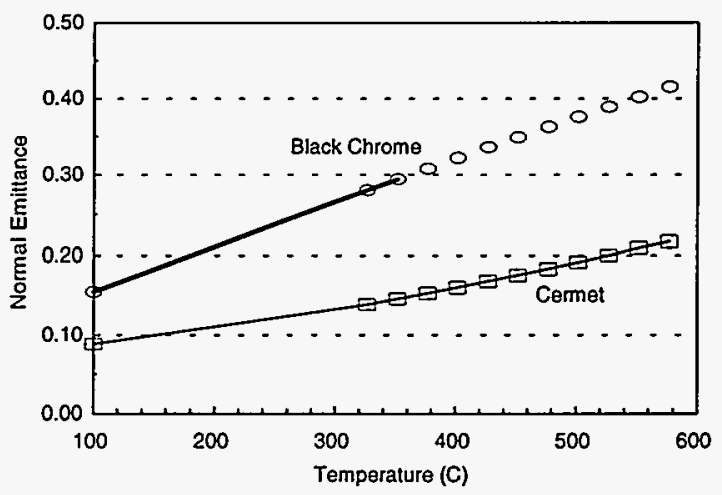

Figure A-2. Black chrome and cermet HCE emittance as a function of temperature.

${ }^{2}$ ASTM E903-82, "Standard test method for solar absorptance, reflectance, and transmittance of materials using integrating spheres," pp. 711-721. 


\section{LS-2 Collector Efficiency Testing}

The National Solar Thermal Test Facility at SNL includes a special collector test platform for testing single-axis-tracking collectors. The test platform uses a rotating table that allows parabolic trough collectors to be tested at normal incidence to the sun, and incorporates a heat transfer fluid loop that can use water or Syltherm 800 as the heat-exchange medium in the collector. The loop uses a heat exchanger and a heater that allow any operating condition necessary for testing to be obtained. Instrumentation is installed to measure fluid inlet and outlet temperature, fluid flow rate, ambient temperature, wind velocity, and direct-normal insolation.

A multistep process is required to determine the thermal efficiency of a parabolic trough collector. First, the optical efficiency of the collector is determined by measuring the collector efficiency while operating at ambient temperature and tracking at normal incidence to the sun. Second, the incident angle modifier (IAM), the impact of off-normal incident beam radiation on optical efficiency, is determined. The IAM is measured by running optical efficiency tests over a range of off-normal incidence angles. In addition, collector thermal losses must be calculated for tracking and non-tracking conditions over the range of fluid operating temperatures. The nontracking (out-of-focus) thermal losses are determined by directly measuring the thermal losses while circulating hot fluid through the collector. The tracking (in-focus) thermal losses are determined by calculating collector efficiency at temperature while tracking at normal incidence to the sun and comparing with the optical efficiency. A detailed empirical model of collector efficiency as a function of fluid temperature, insolation, and solar incidence angle can then be developed based on the test results.

\section{LS-2 Collector Efficiency Test (with Cermet HCE)}

An 8-meter section of an LS-2 collector (one-sixth of a solar collector assembly) and the drive mechanism and control electronics were installed on the SNL rotating collector test platform. The initial tests were conducted using cermet HCEs with good vacuum. The optical efficiency calculated using the above procedure was $73.1 \%$. This optical efficiency compares closely with the LS-2 design. Figure A-3 shows the calculated IAM for angles of $0^{\circ}$ to $90^{\circ}$. The cosine of the incidence angle is also shown in Figure A-3. The cosine effect shows the expected drop in radiation intensity as a function of incidence angle. The IAM includes the cosine losses, changes in collector optical properties at high incidence angles, and losses inherent in the design of the collector. The impacts on optical properties include reduced selective surface absorptance, increased HCE glass reflection, and increased absorption in the HCE and mirror glass. Collector-design-related impacts include HCE shadowing and collector end losses. The IAM data in Figure A-3 have been corrected to exclude test section end losses. The maximum angle experienced by the SEGS projects is approximately $58^{\circ}$ from surface normal, which occurs at solar noon on the winter solstice and corresponds to an IAM of 0.40 .

The collector thermal losses as measured on the test platform are shown in Figure A-4. Note that the in-focus thermal losses are greater than the out-of-focus losses. This is because the thermal losses are a function of the $\mathrm{HCE}$ temperature. Because the in-focus collector is gaining heat, the HCE temperature is greater than that of the fluid. The non-focused collector is losing heat and thus the HCE temperature is lower than the fluid temperature. Thus, the thermal losses of the in-focus collector are greater for a given fluid temperature. 
If the collector thermal losses vary linearly with insolation between the in-focus and out-of-focus tests, the collector efficiency can be calculated for any solar intensity. Figure A-5 shows the resulting collector efficiency at various solar intensities.

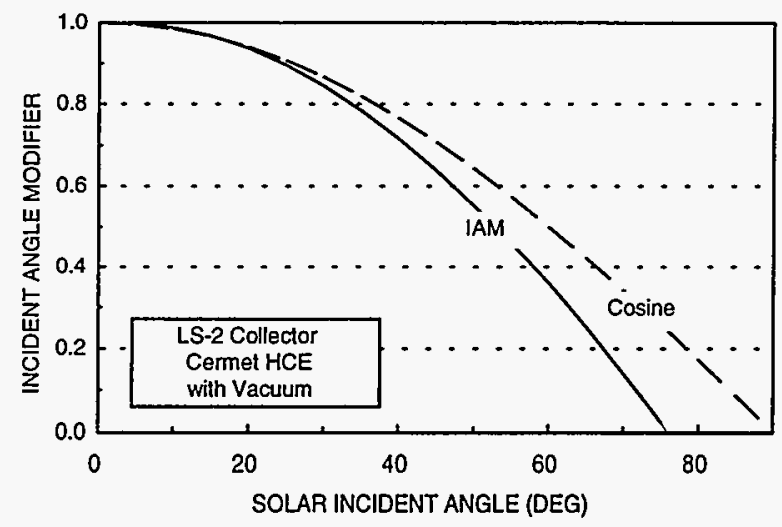

Figure A-3. Incident angle modifier results.

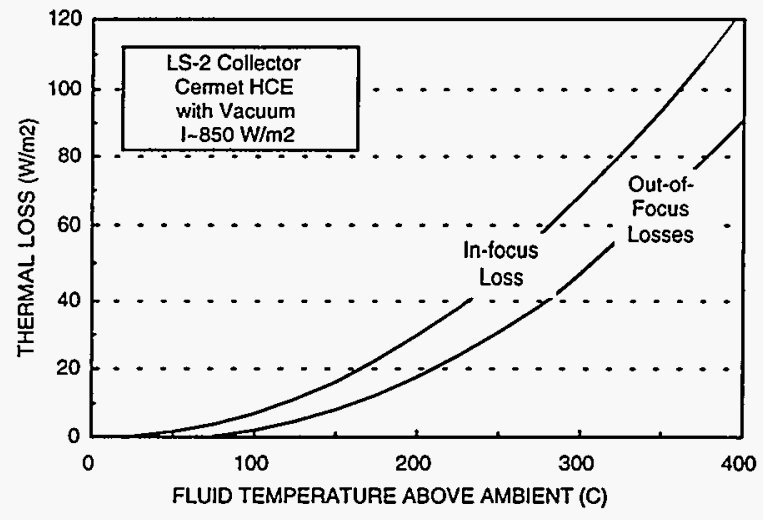

Figure A-4. Collector thermal losses.

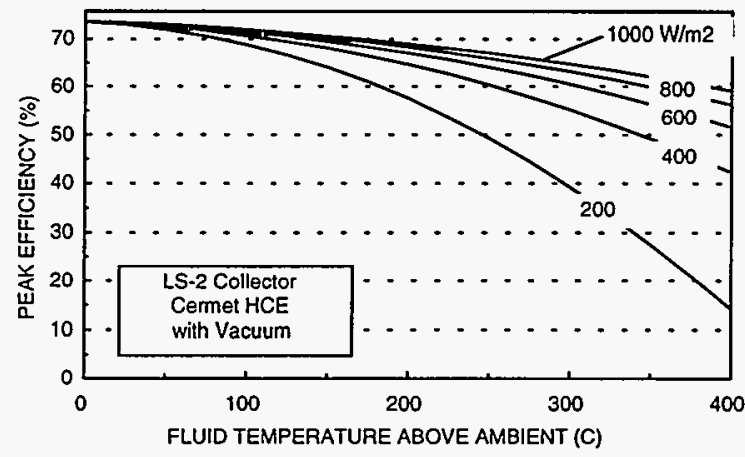

Figure A-5. Collector thermal efficiency. 


\section{Impact of Lost Vacuum and Broken Glass}

The above test procedure was repeated with HCEs with lost vacuum and again with HCEs with broken glass. The lost vacuum condition was achieved by drilling a hole in the bellows that allowed air into the vacuum annulus of the HCEs already installed on the test platform. Likewise, the broken glass condition was achieved by removing the glass from the HCEs following the lost vacuum test. The lost vacuum results mirrored those of the vacuum base case with the exception that the thermal losses were approximately $40 \%$ higher. Wind had very little effect on the efficiency of HCEs with the glass intact; however, once the glass is removed, wind velocity had a significant impact on collector thermal losses. Figure A-6 shows the collector thermal losses for HCEs with vacuum, HCEs that have lost vacuum (air), and broken glass HCEs (bare) for wind speeds of 0,5 , and 10 meters per second.

The heat losses of a bare HCE with no wind were double those of an HCE with vacuum. In a 10-meter-per-second wind, the heat losses from the bare HCE were four or five times that of an $\mathrm{HCE}$ with vacuum. The collector thermal efficiency can be calculated for each HCE condition, as shown in Figure A-7.

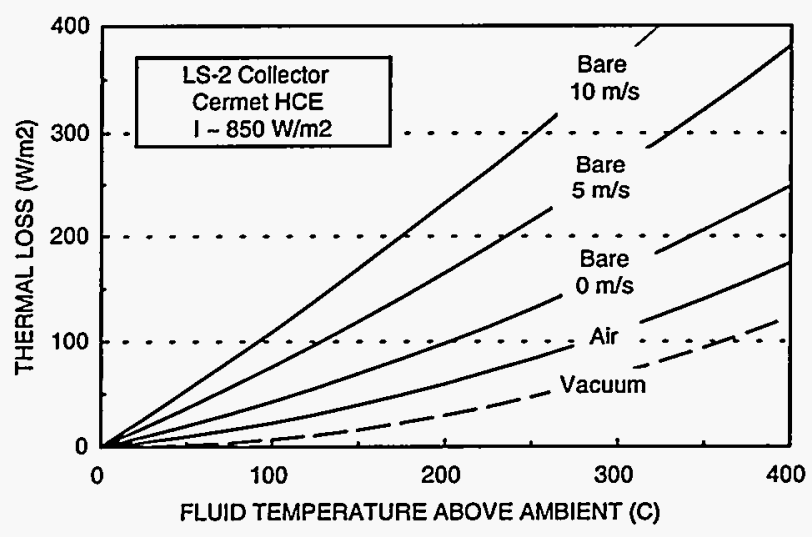

Figure A-6. Collector thermal losses for vacuum, lost vacuum (air), and broken glass (bare) HCEs.

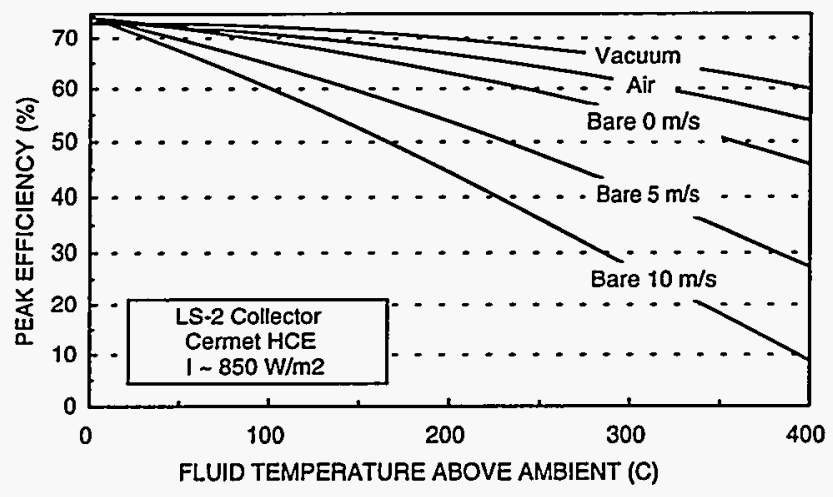

Figure A-7. Collector efficiency for vacuum, lost vacuum (air), and broken glass (bare) HCEs. 


\section{Cermet versus Black Chrome HCEs}

Following the testing of the cermet HCEs, the LS-2 collector was refit with HCEs with a black chrome selective surface. Figure A-8 shows a comparison of thermal efficiency for the collector with black chrome HCEs and with cermet HCEs. The test results show the black chrome to have a higher efficiency at lower temperatures, and the cermet to be better at higher temperatures. These results support the HCE optical property tests results presented in Appendix B.

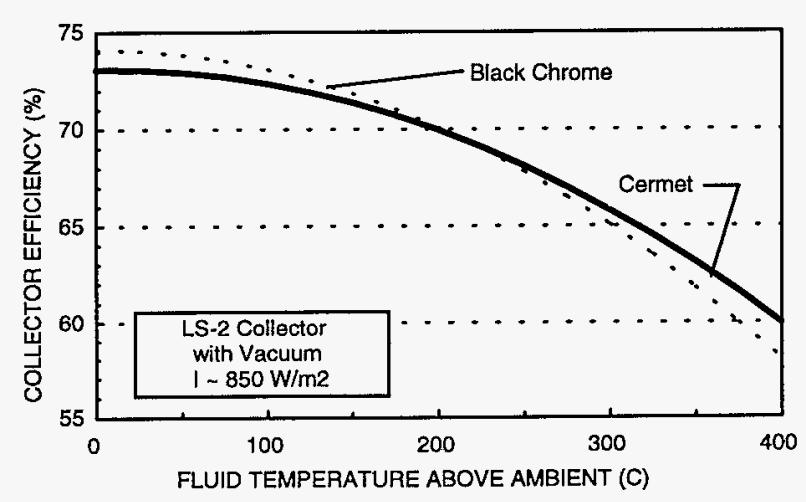

Figure A-8. Collector thermal efficiency for cermet and black chrome HCEs.

\section{Analytical Model}

To gain a detailed understanding of the heat-transfer phenomena, a one-dimensional HCE thermal loss model was developed. The predictions of the model were compared to the test results, and they generally agreed within the uncertainty bands of the experiment. The model and the comparison with the experimental results are fully described in Test Results: SEGS LS-2 Solar Collector, V. Dudley, et al., SAND94-1884, Sandia National Laboratories, Albuquerque, New Mexico, 1994.

\section{Conclusions}

The optical properties of the black chrome selective surface compare closely with the LUZ design data. The cermet selective surface results indicated lower-than-design solar absorptance, but a better-than-design emittance. However, the design emittance is probably within the error tolerances given the size of the sample and the test procedure used.

The LS-2 collector test results agreed closely with the LUZ design optical and collector efficiencies. The test also measured the effects of no vacuum and no glass on collector thermal efficiency. Although loss of vacuum has a significant impact on collector efficiency (approximately a $10 \%$ reduction), it is not clear whether the increased performance justifies the additional cost of a vacuum-insulated HCE tube. Wind was found to have a very strong impact on the performance of HCEs when the glass tube is broken. Reglazing tubes where the glass has broken would likely have an attractive payback (see Appendix B). 


\section{Appendix B \\ Heat Collection Element Refurbishment}

The glass envelope on heat collection elements (HCEs) periodically breaks because of a number of causes that have been discussed in detail elsewhere. Tests described in Appendix A have shown that heat losses from HCEs in this condition can be markedly reduced by reglazing the HCEs with a new glass envelope. In this case, the annular space between the glass and metal tube will not be evacuated, but external convection heat transfer losses, which can be very high in windy conditions, will be essentially eliminated. While the improvement would only be approximately $5 \%$ to $10 \%$ in calm conditions, the effect in windy conditions would be very high. A simple reglazing procedure was developed for this purpose by Industrial Solar Technologies of Golden, Colorado (see Figure B-1). Widespread implementation in the Kramer Junction, California, solar fields was planned to test the durability and usefulness of this approach.

Initially, six prototypes were installed at the Solar Electric Generating Systems VI plant in early 1995. This installation was done in the solar field test loop in that plant with the hope that the effects of reduced heat losses could be measured. The test-loop data proved insensitive to the effects of such a small change on performance because of the small number of HCEs that were affected and the relatively small improvement compared to full-loop performance.

Observations on the first prototypes resulted in minor design changes by Industrial Solar Technologies to improve field installation and durability. An additional 80 units were delivered to the plants by mid-summer for installation. Broken HCEs are replaced at the plants only when there are enough failures in a loop to warrant shutting it down for this procedure. The reglazing units are used before HCEs in a loop are replaced. Once this takes place, the reglazing unit can be used elsewhere in the solar field on a regular basis.

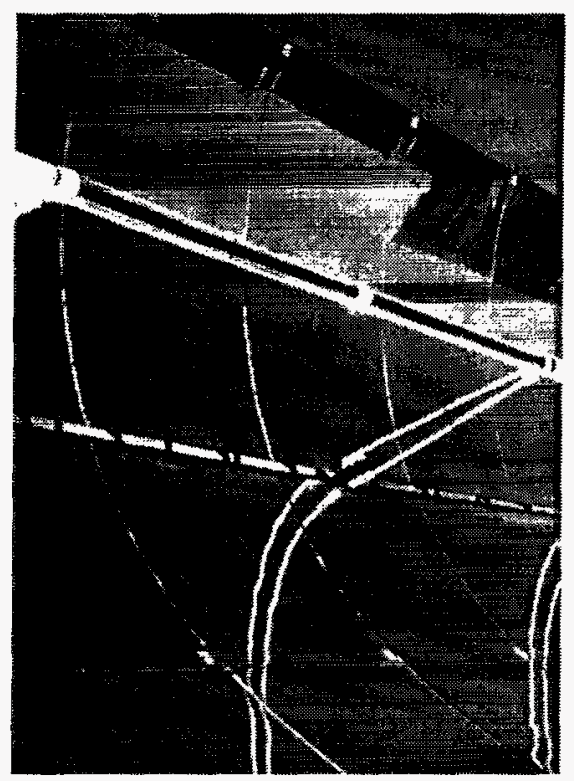

Figure B-1. The reglazing kit consists of four glass sections that are held together with adhesives and springs. 
This page intentionally left blank. 


\section{Appendix C . \\ Heat Collection Element Coating Development}

The heat collection elements (HCEs) are the receivers of the solar energy, i.e., the units to which the direct-normal insolation is focused by the mirrors. A standard HCE (shown in Figure C-1) consists of the stainless steel tube, selective cermet surface, and glass outer envelope. The annular space between the tube and glass envelope is evacuated in the standard design, virtually eliminating heat loss across the space by conduction or convection. Radiation losses are greatly reduced by the very low emissivity of the selective surface that, conversely, has a very high incident radiation absorptivity.

In 1996, SOLEL Solar Systems of Israel, the manufacturers of the HCE, modified the cermet selective surface, achieving a lower emissivity. Table $\mathrm{C}-1$ below compares the old and new characteristics.

Sandia National Laboratories (SNL) performed a detailed evaluation of this surface, as described below.

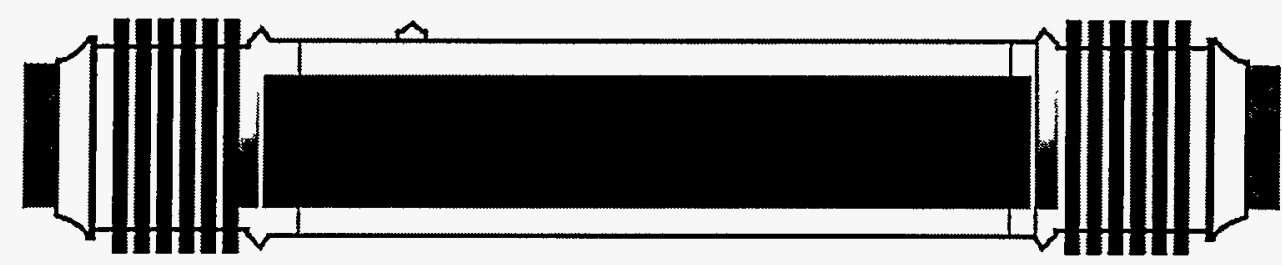

Figure C-1. Heat collection element.

Table C-1. HCE Characteristics

\begin{tabular}{|l|c|c|}
\hline \multicolumn{1}{|c|}{ Property } & Nominal (1995) & Improved (1996) \\
\hline Absorptivity & 0.965 & 0.965 \\
\hline Emissivity at $350^{\circ} \mathrm{C}$ & 0.15 & $<0.10$ \\
\hline
\end{tabular}

\section{SNL Testing of Cermet Provided by SOLEL Solar Systems Limited}

The results of the optical properties characterization and a limited accelerated thermal aging (in air) study of three cermet coatings produced by SOLEL Solar Systems Limited of Israel are presented in this document. The goal of this limited study was to assess the relative optical properties and thermal stability of these coatings. Thermal exposure temperatures (in air) of $325^{\circ} \mathrm{C}, 375^{\circ} \mathrm{C}, 400^{\circ} \mathrm{C}$, and $435^{\circ} \mathrm{C}$ were used to assess the coatings durability.

The three cermet coatings consisted of (1) an unexposed (new) tube section from the most recent KJC Operating Company (KJCOC) heat collector element purchase, obtained from Gilbert Cohen; this material served as the control samples, (2) three samples from SOLEL lot M13596B, and (3) three samples from SOLEL lot M9596A. The samples provided by SOLEL were prepared on polished stainless steel sheet substrates. 


\section{Measurement Methods}

The hemispherical reflectance properties, covering the wavelength range from 265 nanometers $(\mathrm{nm})$ to 2,500 nm, were measured using a Cary Model 5E spectrophotometer equipped with a Labsphere integrating sphere accessory. All reflectance data obtained using this instrument were calibrated according to the National Institute of Standards and Technology standards and should have a measurement uncertainty of \pm 0.005 reflectance units $(1.000$ reflectance units $=100 \%$ reflectance).

A second instrument, a portable infrared reflectometer, Model DB-100 manufactured by GierDunkle Instrument Company, was used to measure the infrared characteristics. This particular instrument uses a rotating heated cavity and a detector with a sensitivity range from $3000 \mathrm{~nm}$ to $30000 \mathrm{~nm}$ to measure the total normal infrared reflectance of samples placed over the measurement port. As designed, the instrument measures the total normal infrared reflectance, $\rho_{n}\left(100^{\circ} \mathrm{C}\right)$, for a $100^{\circ} \mathrm{C}$ blackbody with a peak wavelength, $\lambda_{\text {peak }}^{100 \mathrm{C}}$, of $7769 \mathrm{~nm}$. A filter is placed in the optical path to allow an additional infrared reflectance measurement, $\rho_{n}\left(300^{\circ} \mathrm{C}\right)$, approximating a $300^{\circ} \mathrm{C}$ blackbody distribution with a peak wavelength, $\lambda_{\text {pcak }}^{300 \mathrm{C}}$, of $5058 \mathrm{~nm}$. For opaque materials, the total normal DB-100 measured emittance, $\varepsilon_{n}\left(100^{\circ} \mathrm{C}\right.$ or $\left.300^{\circ} \mathrm{C}\right)$ is then:

$$
\varepsilon_{n}^{\prime}\left(100^{\circ} \mathrm{C} \text { or } 300^{\circ} \mathrm{C}\right)=1.000-\rho_{\mathbf{n}}\left(100^{\circ} \mathrm{C} \text { or } 300^{\circ} \mathrm{C}\right)
$$

Merging of the reflectance data from these two instruments enables optical characterization of the material over the combined wavelength range, $265 \mathrm{~nm}$ to $30,000 \mathrm{~nm}$ (the $30,000 \mathrm{~nm}$ value was assumed equal to the $\lambda_{\text {peak }}^{100 c}$ value for these materials) with an estimated measurement uncertainty of \pm 0.05 units. Specific techniques regarding the measurement of optical properties using these instruments are outlined by the American Society of Testing and Materials in the documents Solar Absorptance, Reflectance, and Transmittance Using Integrating Spheres, ASTM E903-82, and Total Normal Emittance of Surfaces Using Inspection-Meter Techniques, ASTM E408-71. Note that the reflectance or absorptance values listed were measured under near-normal incident angle conditions.

Using the measured reflectance data, the solar averaged hemispherical absorptance, $\alpha_{s}(2 \pi)$, value was calculated using expression (2):

$$
\alpha_{s}(2 \pi)=\frac{\int_{\lambda_{1}}^{\lambda_{2}} \operatorname{SE}_{\lambda}\left[1-\rho_{\lambda}(2 \pi)\right] \mathrm{d} \lambda}{\int_{\lambda_{1}}^{\lambda_{2}} \mathrm{SE}_{\lambda} \mathrm{d} \lambda},
$$

where $\mathrm{SE}_{\lambda}$ is the irradiance value at wavelength $\lambda$ for the standard air mass 1.5 total normal solar energy distribution, ASTM E892-87, and $\rho_{\lambda}(2 \pi)$ is the measured reflectance at wavelength $\lambda$. The integration interval ranged from $\lambda_{1}=300 \mathrm{~nm}$ to $\lambda_{2}=2,500 \mathrm{~nm}$.

The blackbody averaged reflectance, $\mathrm{R}_{\mathrm{BB}}(T)$, value, and was calculated using the measured data over the entire wavelength region by combining the data from both the Cary and the Gier-Dunkle instruments and expression (3): 


$$
\mathrm{R}_{\mathrm{BB}}(T)=\frac{\int_{\lambda_{3}}^{\lambda_{4}} \mathrm{BB}_{\lambda}(T)\left[1-\rho_{\lambda}(2 \pi)\right] \mathrm{d} \lambda}{\int_{\lambda_{3}}^{\lambda_{4}} \mathrm{BB}_{\lambda}(T) \mathrm{d} \lambda},
$$

where temperature $T$ is in degrees Kelvin, $\mathrm{K}$. The variable $\mathrm{BB}_{\lambda}(T)$ represents the Planck distribution value for temperature $T(\mathrm{~K})$ and wavelength $\lambda$ calculated using equation (4):

$$
\mathrm{BB}_{\lambda}(T)=\left(2 \pi \frac{\mathrm{c}^{2} \mathrm{~h}}{\lambda^{5}}\right)\left[\exp \left\{\frac{\mathrm{ch}}{\lambda \mathrm{k}_{\mathrm{B}} T}\right\}^{-1}\right]^{-1},
$$

where $\mathrm{c}=$ speed of light, $\mathrm{h}=$ Planck's constant, and $\mathrm{k}_{\mathrm{B}}=$ Boltzman's constant. The integration interval for equation (3) was from $\lambda_{3}=265 \mathrm{~nm}$ and $\lambda_{4}=30,000 \mathrm{~nm}$. The normal emittance, $\varepsilon_{\mathrm{n}}(T)$, at temperature $T$ was calculated using equation (5):

$$
\varepsilon_{\mathrm{n}}(T)=1.000-\mathrm{R}_{\mathrm{BB}}(T) .
$$

\section{Measured Data}

Table $\mathrm{C}-1$ lists the calculated $\alpha_{\mathrm{s}}(2 \pi)$ and $\varepsilon_{\mathrm{n}}(T)$ values typical for the three cermet materials. Note that the "new" cermet coatings exhibited significantly lower emittance values $(0.04$ compared to 0.08 ) than the current HCE cermet; the solar absorptance values ranged from 0.949 to 0.966 .

\section{Results for Samples Aged at Various Temperatures Up to $435^{\circ} \mathrm{C}$}

To assess the relative durability of the coatings, accelerated thermal exposure testing, in air, was done at four different temperatures: $325^{\circ} \mathrm{C}, 375^{\circ} \mathrm{C}, 400^{\circ} \mathrm{C}$, and $435^{\circ} \mathrm{C}$. Initially, a set of samples was aged for various times at $325^{\circ} \mathrm{C}, 375^{\circ} \mathrm{C}$, and $435^{\circ} \mathrm{C}$ to determine an exposure temperature that resulted in some observable coating degradation. The optical properties where measured before and after each exposure time and condition and are listed in Table C-2. Notice that only the emittance values measured using the Gier-Dunkle DB-100 instrument are included in the table (the total normal emittance values calculated using equations (3), (4), and (5) are not listed, but can be provide upon request). These data indicate that no optical properties changes occurred, within measurement uncertainties, for the samples tested. However, when the sample surfaces were examined using optical microscopy, all samples exhibited significant spallation of the outer dielectric layer. Micrographs of these three samples are included in Figures C-2 through C-4. 
Table C-1. Calculated Solar Absorptance and Total Normal Emittance Values, as a Function of Temperature for Typical SOLEL Cermet Materials, as Received

\begin{tabular}{|c|c|c|c|c|}
\hline ID & Condition & $\alpha_{\mathbf{S}}(2 \pi)$ & Temperature $\left({ }^{\circ} \mathbf{C}\right)$ & $\boldsymbol{\varepsilon}_{\mathbf{n}}(\mathbf{T})^{*}$ \\
\hline KJCOC & As Received & 0.966 & 250 & 0.072 \\
Control & & & 275 & 0.074 \\
$96-164-1 C$ & & & 300 & 0.076 \\
& & & 325 & 0.078 \\
& & & 350 & 0.081 \\
& & & 375 & 0.083 \\
& & 400 & 0.086 \\
\hline SOLEL & As Received & 0.960 & 250 & 0.038 \\
Lot M13596B & & & 275 & 0.039 \\
Sample \#1 & & & 300 & 0.040 \\
& & & 325 & 0.041 \\
& & & 350 & 0.042 \\
& & & 375 & 0.044 \\
SOLEL & As Received & 0.949 & 250 & 0.046 \\
\hline Lot M9596A & & & 275 & 0.038 \\
Sample \#4 & & & 300 & 0.039 \\
& & & 325 & 0.040 \\
& & & 375 & 0.041 \\
& & & 400 & 0.042 \\
& & & & 0.044 \\
& & & 30 & 0.046 \\
\hline
\end{tabular}

*Normal emittance as a function of temperature was calculated using equations (3), (4), and (5).

Table C-2. SOLEL Cermet Samples Thermal Aging (In Air) at $325^{\circ} \mathrm{C}, 375^{\circ} \mathrm{C}$, and $435^{\circ} \mathrm{C}$ Measured Solar Absorptance and DB-100 Measured Thermal Emittance

\begin{tabular}{|c|c|c|c|c|c|c|}
\hline ID & Time/Temp $\left({ }^{\circ} \mathrm{C}\right)$ & $\alpha_{\mathrm{s}}(2 \pi)$ & $\varepsilon_{\mathrm{n}}^{\prime}\left(100^{\circ} \mathrm{C}\right)$ & $\Delta \varepsilon_{\mathrm{n}}^{\prime}\left(100^{\circ} \mathrm{C}\right)$ & $\varepsilon_{\mathrm{n}}^{\prime}\left(300^{\circ} \mathrm{C}\right)$ & $\Delta \varepsilon_{\mathrm{n}}^{\prime}\left(300^{\circ} \mathrm{C}\right)$ \\
\hline KJCOC & As Received & 0.966 & 0.062 & --- & 0.101 & --- \\
Control & 96 hours $/ 435^{\circ} \mathrm{C}$ & 0.966 & 0.083 & 0.021 & 0.118 & 0.017 \\
$96-164-1 \mathrm{C}$ & & & & & & \\
\hline SOLEL & As Received & 0.960 & 0.036 & --- & 0.047 & --- \\
Lot M13596B & 139 hours $325^{\circ} \mathrm{C}$ & 0.960 & 0.033 & -0.003 & 0.046 & -0.001 \\
Sample \#1 & +50 hours $/ 375^{\circ} \mathrm{C}$ & 0.960 & 0.034 & -0.002 & 0.043 & -0.004 \\
& +139 hours $375^{\circ} \mathrm{C}$ & 0.960 & 0.033 & -0.003 & 0.043 & -0.004 \\
& +96 hours $/ 435^{\circ} \mathrm{C}$ & 0.960 & 0.043 & 0.009 & 0.045 & +0.002 \\
\hline SOLEL & As Received & 0.949 & 0.037 & --- & 0.046 & --- \\
Lot M9596A & 139 hours $325^{\circ} \mathrm{C}$ & 0.949 & 0.034 & -0.003 & 0.042 & -0.004 \\
Sample \#4 & +50 hours $375^{\circ} \mathrm{C}$ & 0.949 & 0.036 & -0.001 & 0.039 & -0.007 \\
& +139 hours $375^{\circ} \mathrm{C}$ & 0.949 & 0.035 & -0.002 & 0.042 & -0.004 \\
& +96 hours $/ 435^{\circ} \mathrm{C}$ & 0.949 & 0.040 & 0.004 & 0.050 & 0.004 \\
\hline
\end{tabular}


In the micrographs below, notice that the $\mathrm{KJCOC}$ control sample, shown in Figure C-2, exhibits a much rougher surface finish than the two SOLEL samples prepared on the stainless steel sheet substrates. SOLEL sample lot M13596B-\#1, shown in Figure C-3, appears to have significantly more spallation sites (bright areas where the dielectric layer is removed exposing the underlying metallic layer) than sample lot M9596A-\#4, shown in Figure C-4. For cermet coatings that use molybdenum for the metal reflectance layer, any spallation is considered a potentially serious problem. In the presence of oxygen, which would occur if the vacuum in the HCE annulus was breached to the ambient environment, molybdenum forms a volatile oxide that would condense on the inside surface of the glass envelope eventually resulting in a "fluorescent" HCE."

The large number of spallation sites observed on the KJCOC control sample were located in areas either at the peak or in the valley of the residual polishing features. The spallation sites on the SOLEL samples were observed in locations along residual surface scratches or in locations of probable surface particulate contamination. Typically, the nucleation site for the spallation area correlates to surface defects were stress concentrations occur in the film. Other cermet materials that have been thermally aged at temperatures above the coating design temperature have shown similar spallation of the dielectric layer. For these reasons and after discussing some of these results with A. Brenmiller at SOLEL, another set of samples was thermal aged in air at $400^{\circ} \mathrm{C}$ and is discussed below.

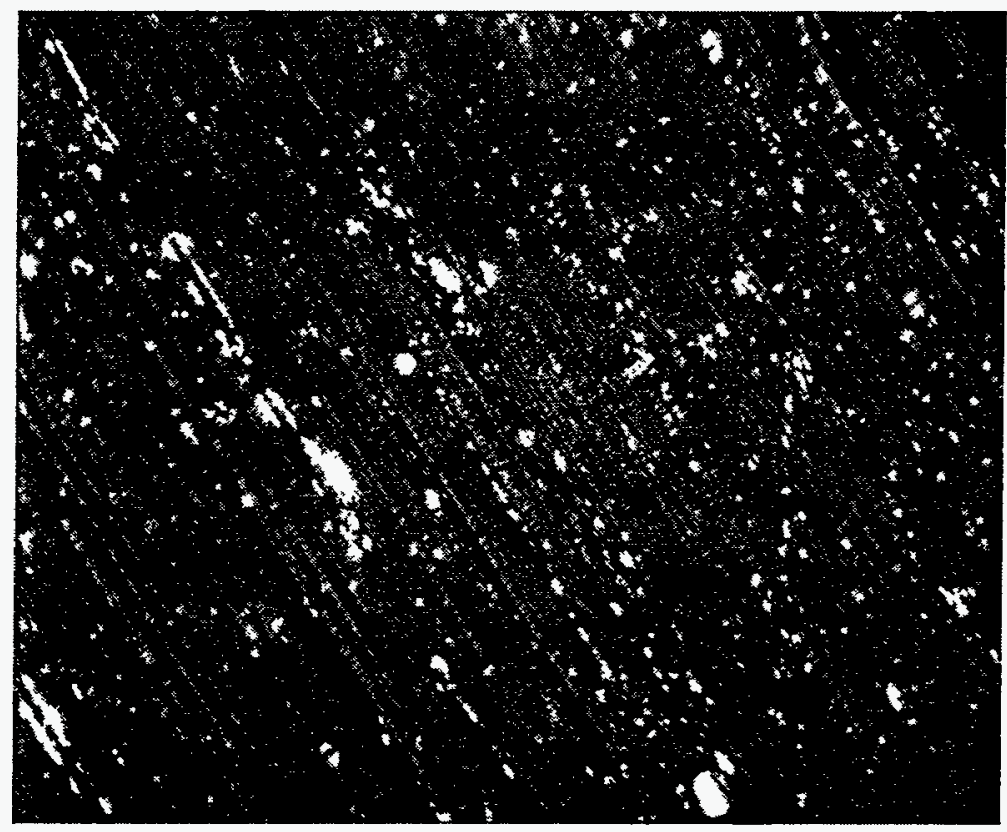

Figure C-2. Photomicrograph (250X) of KJCOC control cermet sample 96-164-1C after thermal exposure in air for 139 hours at $325^{\circ} \mathrm{C}+189$ hours at $375^{\circ} \mathrm{C}+96$ hours at $435^{\circ} \mathrm{C}$.

\footnotetext{
- Fluorescent HCEs can be found in the California solar fields at Kramer Junction and Harper Lake.
} 


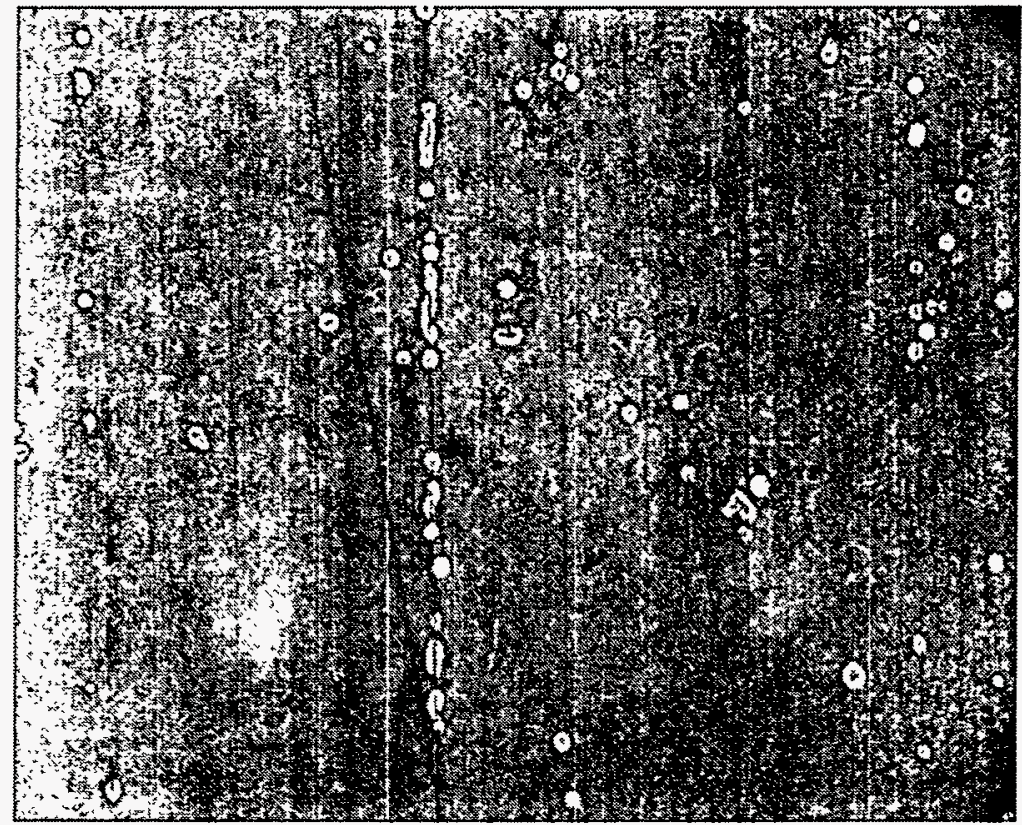

Figure C-3. Photomicrograph (80X) of SOLEL cermet lot M13596B sample \#1 after thermal exposure in air for 139 hours at $325^{\circ} \mathrm{C}+189$ hours at $375^{\circ} \mathrm{C}+96$ hours at $435^{\circ} \mathrm{C}$.

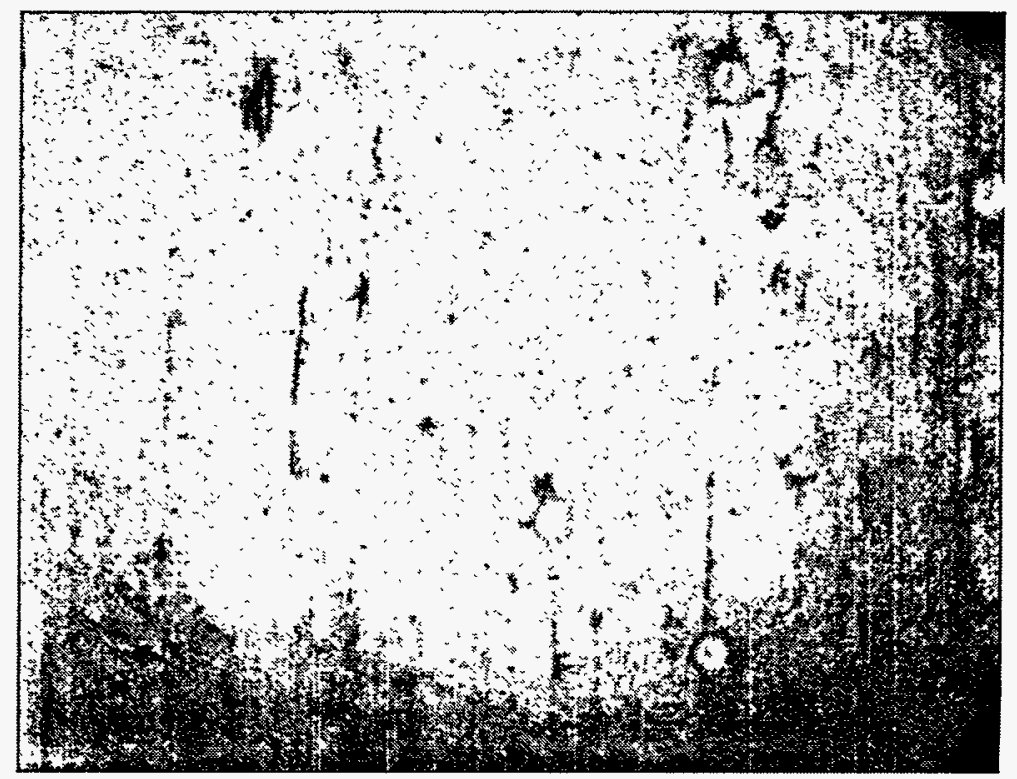

Figure C-4. Photomicrograph (250X) of SOLEL cermet lot M9596A sample \#4 after thermal exposure in air for 139 hours at $325^{\circ} \mathrm{C}+189$ hours at $375^{\circ} \mathrm{C}+96$ hours at $435^{\circ} \mathrm{C}$. 


\section{Results for Samples Aged at $400^{\circ} \mathrm{C}$}

Table C-3 lists the measured optical properties results for the cermet sample set thermally aged at $400^{\circ} \mathrm{C}$. Only the emittance values measured using the Gier-Dunkle DB-100 instrument are included in the table. These data also indicate that no optical properties changes occurred, within measurement uncertainties, for the samples tested.

In the micrographs that follow, the KJCOC control sample (shown in Figure C-5) exhibits a much rougher surface finish than the two SOLEL samples prepared on the stainless steel sheet substrates. SOLEL sample lot M13596B-\#3, shown in Figure C-6, again appears to have significantly more spallation sites (bright areas where the dielectric layer is removed exposing the underlying metal layer) than sample lot M9596A-\#5, shown in Figure C-7.

Table C-3. SOLEL Cermet Samples Thermal Aging (In Air) at $400^{\circ} \mathrm{C}$ Measured Solar Absorptance and DB-100 Measured Thermal Emittance

\begin{tabular}{|c|c|c|c|c|c|c|}
\hline ID & $\begin{array}{c}\text { Time/Temp } \\
\left({ }^{\circ} \mathrm{C}\right)\end{array}$ & $\alpha_{\mathrm{s}}(2 \pi)$ & $\varepsilon_{\mathrm{n}}^{\circ}\left(100^{\circ} \mathrm{C}\right)$ & $\Delta \varepsilon_{\mathrm{n}}^{\prime}\left(100^{\circ} \mathrm{C}\right)$ & $\varepsilon_{\mathrm{n}}^{\prime}\left(300^{\circ} \mathrm{C}\right)$ & $\Delta \varepsilon_{\mathrm{n}}\left(300^{\circ} \mathrm{C}\right)$ \\
\hline KJCOC & As Received & 0.965 & 0.060 & --- & 0.100 & -- \\
Control & 140 hours $/ 400^{\circ} \mathrm{C}$ & 0.965 & 0.076 & 0.016 & 0.122 & 0.022 \\
96-164-1A & 290 hours $/ 400^{\circ} \mathrm{C}$ & 0.965 & 0.086 & 0.026 & 0.135 & 0.035 \\
\hline SOLEL & As Received & 0.959 & 0.036 & --- & 0.047 & -- \\
Lot M13596B & 140 hours $/ 400^{\circ} \mathrm{C}$ & 0.959 & 0.035 & -0.001 & 0.040 & -0.007 \\
Sample \#3 & 290 hours $/ 400^{\circ} \mathrm{C}$ & 0.959 & 0.036 & 0.000 & 0.042 & -0.005 \\
\hline SOLEL & As Received & 0.950 & 0.037 & -- & 0.046 & --- \\
Lot M9596A & 140 hours $/ 400^{\circ} \mathrm{C}$ & 0.950 & 0.034 & -0.003 & 0.040 & -0.006 \\
Sample \#5 & 290 hours $/ 400^{\circ} \mathrm{C}$ & 0.950 & 0.036 & -0.001 & 0.043 & -0.003 \\
\hline
\end{tabular}

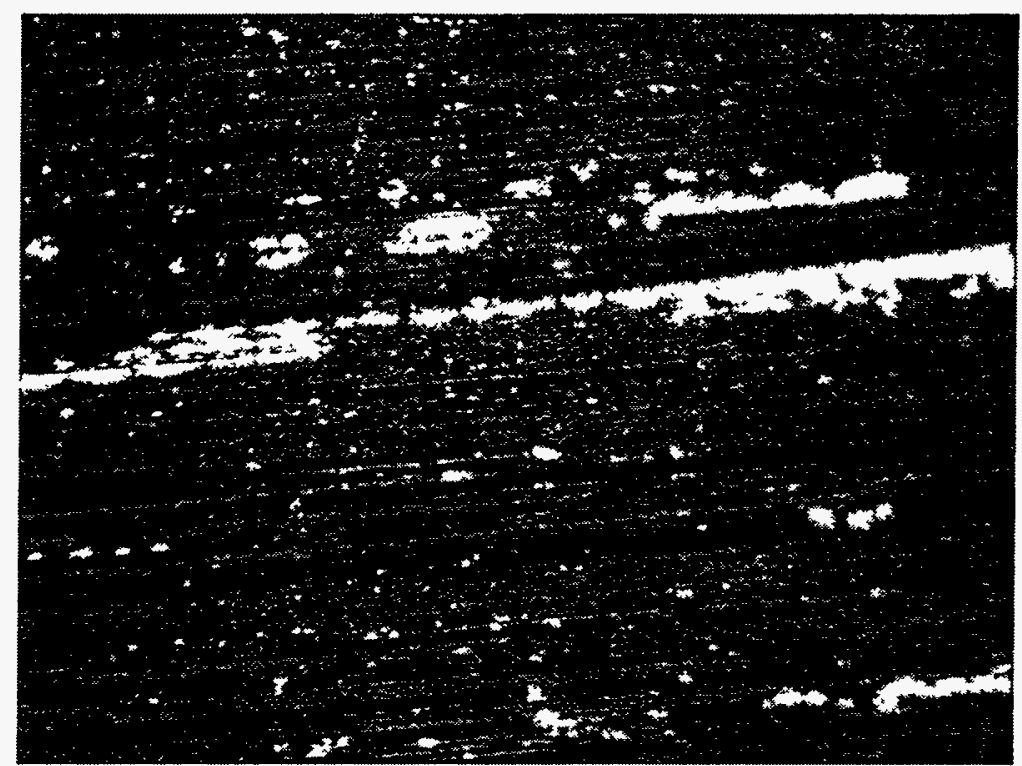

Figure C-5. Photomicrograph (250X) of KJCOC control cermet sample 96-164-1A after thermal exposure in air for 290 hours at $400^{\circ} \mathrm{C}$. 


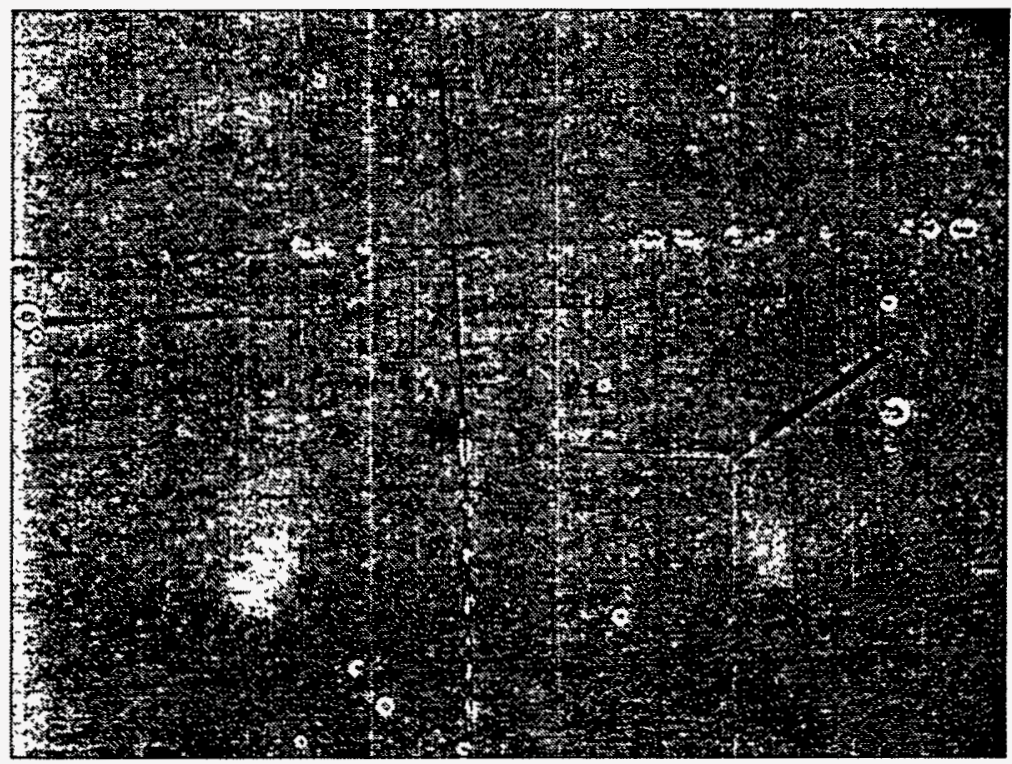

Figure C-6. Photomicrograph (80X) of SOLEL cermet lot M13596B sample \#3 after thermal exposure in air for 290 hours at $400^{\circ} \mathrm{C}$.

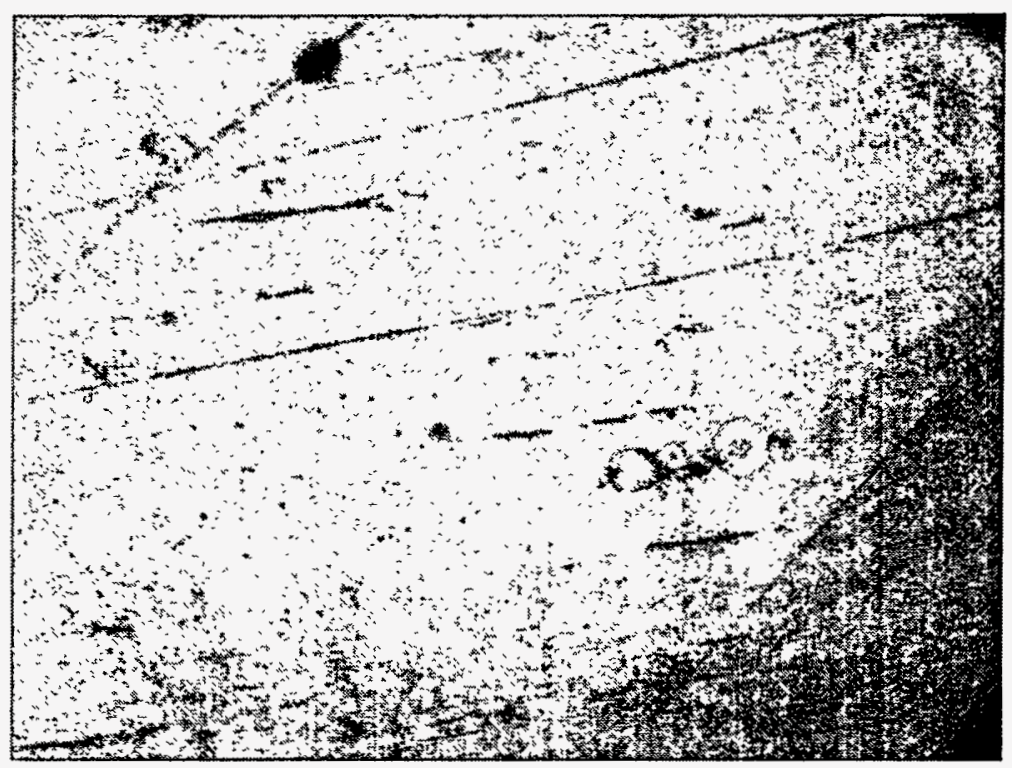

Figure C-7. Photomicrograph (250X) of SOLEL cermet lot M9596A sample \#5 after thermal exposure in air for 290 hours at $400^{\circ} \mathrm{C}$.

\section{Conclusions}

The measured optical properties for the three cermet coatings tested, both before and after thermal exposure (in air), indicated no significant changes for the various aging temperatures up to $435^{\circ} \mathrm{C}$. The $\mathrm{KJCOC}$ control sample exhibited an $\alpha_{s}(2 \pi)$ value of 0.966 with an $\varepsilon_{\mathrm{n}}\left(350^{\circ} \mathrm{C}\right)$ value of 0.08 . The "new" cermet coatings exhibited $\alpha_{s}(2 \pi)$ values of 0.96 and 0.95 and $\varepsilon_{\mathrm{n}}\left(350^{\circ} \mathrm{C}\right)$ values of 0.04 and 0.04 , for sample lots M13596B and M9596A, respectively. 
Using optical microscopy to observe the sample surfaces after thermal aging, there appears to be a subtle difference in thermal stability between the samples from lot M9596A and lot M13596B. For both the $400^{\circ} \mathrm{C}$ and $435^{\circ} \mathrm{C}$ exposure conditions, lot M9596A exhibited fewer locations of coating spallation than lot M13596B. The KJCOC cermet samples exhibited the worst spallation under all conditions. Reasons for this behavior are currently unknown, but could be related to surface roughness, substrate preparation, surface particulate contamination, coating deposition temperature, and cermet layer composition and/or stoichiometry.

A direct comparison between the three coatings studied, deposited on substrates with such differing surface roughness, is not possible; however, spallation has been previously observed on other cermet materials exposed to temperatures greater than the design temperature. As previously observed and stated, any spallation of the dielectric layer on cermet coatings that use molybdenum for the metal reflectance layer is considered a potentially serious long-term problem in a vacuumless environment at elevated temperatures because of the formation of a volatile oxide in the presence of oxygen. 
This page intentionally left blank. 


\section{Appendix D \\ Vacuumless Heat Collection Element}

Development of the improved selective surface has led KJC Operating Company (KJCOC) to postulate the advantages of a design modification to eliminate the loss-of-vacuum failure mechanism by producing a vacuumless heat collection element ( $\mathrm{HCE})$. That is, the reduction in radiation losses gained from the lower emissivity could partially offset the increase in heat losses incurred by elimination of the vacuum, and the lower cost and increased reliability of the modified HCE might justify a change that would also result in reduced solar field maintenance costs.

Work was conducted at $\mathrm{KJCOC}$ to conceive the configuration of a vacuumless $\mathrm{HCE}$, simplifying the design as much as possible. The selected vacuumless design is illustrated in Figures D-1, D-2, and D-3. Note that the outer glass envelope is fixed on one end with a tight seal on the other end that permits axial movement during thermal expansion or contraction (called a "slide support"). The potential pros and cons of this approach include the following:

- Reduced emissivity, increasing performance by $3 \%$ to $4 \%$.

- Elimination of expansion bellows, increasing the effective absorber length and improving performance by $3 \%$.

- Elimination of vacuum, decreasing performance by up to $15 \%$.

- Lower initial unit cost by approximately $40 \%$ because of simpler configuration and manufacturing (e.g., no bakeout is required).

- No need for replacement over the lifetime of the plant because if the glass breaks, it can be replaced in the field.

- Success of this approach is based on long-term durability of the selective surface properties in air rather than in a vacuum, a characteristic that has not yet been demonstrated.

SOLEL Solar Systems was chosen to supply the vacuumless HCE. Two hundred units were delivered in March 1997 for use in special test loops established to test their effectiveness.

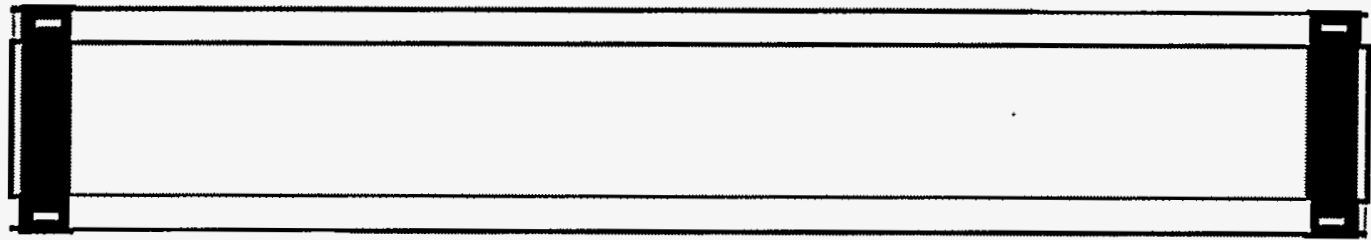

Figure D-1. Drawing of a vacuumless HCE.

\footnotetext{
- In the conventional evacuated $\mathrm{HCE}$, the glass is welded to expansion bellows with a glass-to-metal seal.
} 


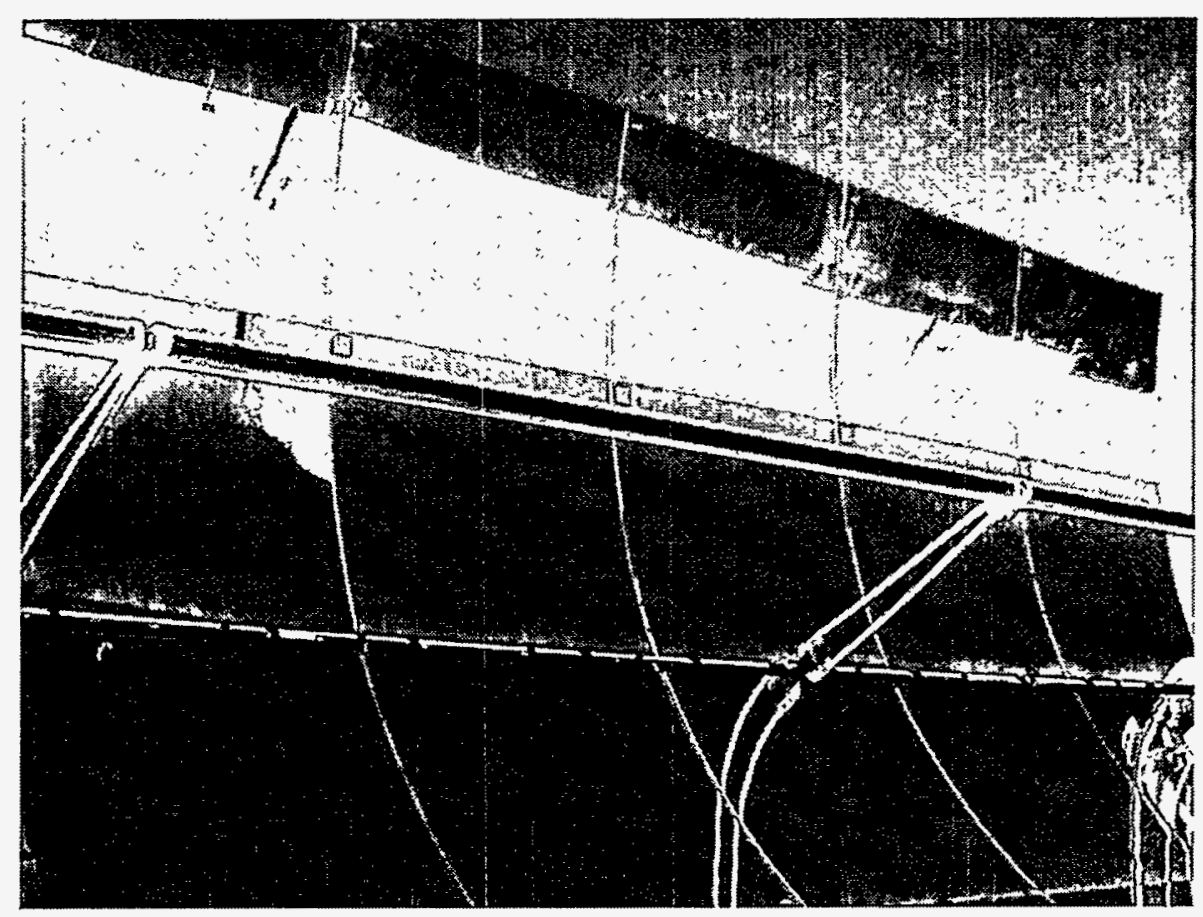

Figure D-2. Vacuumless HCE installed on collector.

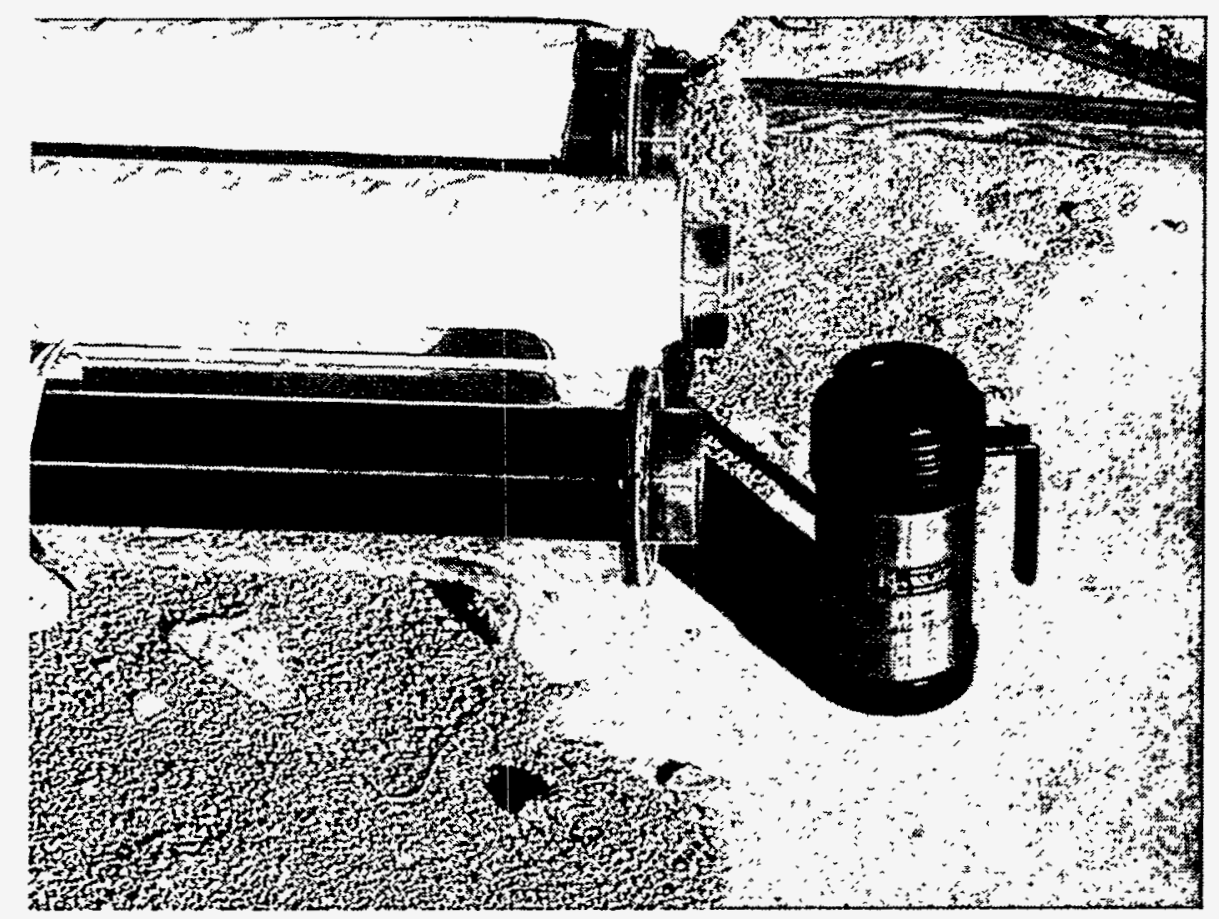

Figure D-3. Close-up of vacuumless HCE. 


\section{Test Description}

Three test loops in the southeast quadrant of the Solar Electric Generating Systems III plant were established. All solar collector assemblies in the loops were brought to good operating condition as required, including all mirrors and HCEs. Flow and temperature instrumentation were installed to monitor performance data and logged using a Campbell 21X data logger.

The three loops consist of the following:

- Reference loop (rows 5 and 6) - standard cermet coating; evacuated

- Vacuumless loop (rows 7 and 8) - new cermet coating; no vacuum

- High-performance loop (rows 9 and 10) - new cermet coating; evacuated

\section{Test Results}

The thermal deliveries of the three test loops are compared in Figure D-4. The figure shows that evacuated tubes with new cermet ("Super Loop") have an improved thermal delivery, relative to evacuated tubes with standard cermet ("Cermet Loop"), of more than $4 \%$. The figure also shows that the thermal delivery of the vacuumless tubes employing new cermet ("VL Adj") is approximately $11 \%$ below the evacuated tubes with new cermet.

The vacuumless loop performed near expected levels and could be incorporated into a future plant once more research and development occurs to perfect the design. The main problem is to prevent binding of the slide support. At first, the slide supports were not adjusted correctly and some glass envelopes broke because the supports were not sliding. Even adjusted correctly, they occasionally "hang up." Still to be determined is whether the cermet coating will degrade after extended exposure to air.

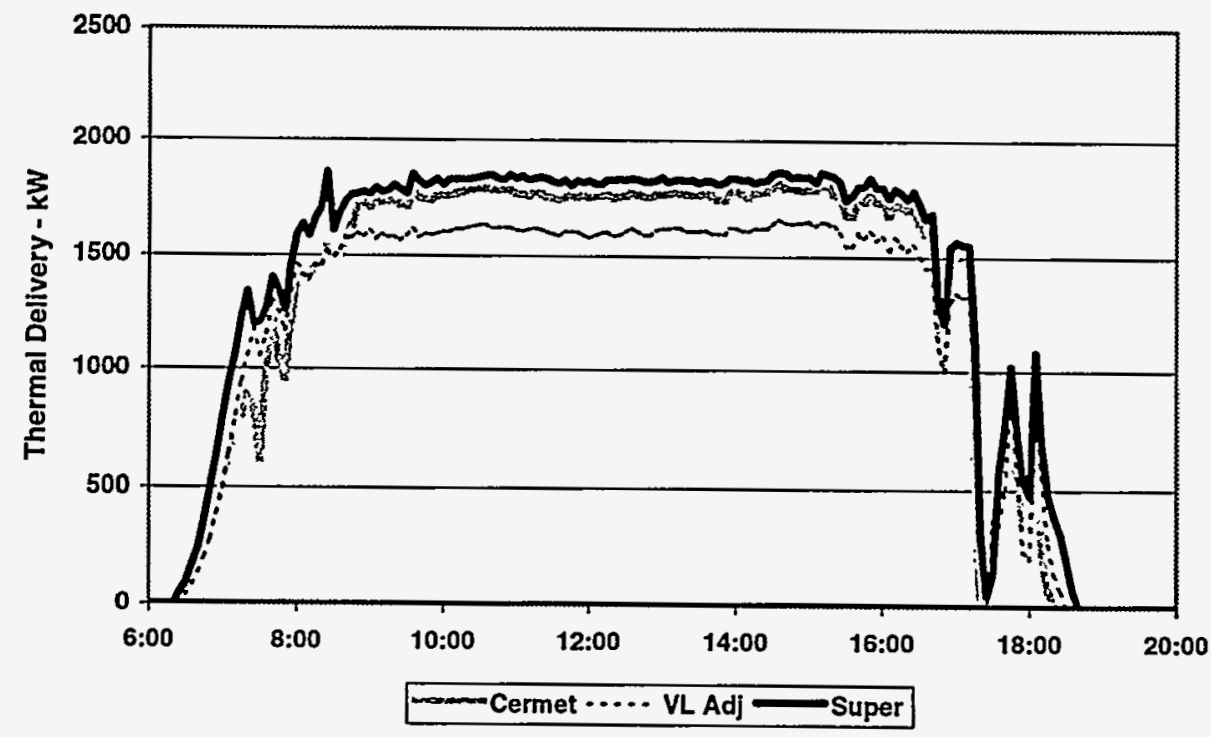

Figure D-4. Comparison of thermal delivery of three test loops. The curve labeled "Cermet" is the reference loop, "VL Adj" is the vacuumless loop, and "Super" is the high-performance loop. 
This page intentionally left blank. 


\section{Appendix E MirROR CLEANLINESS}

\section{Background}

Solar field performance is directly dependent on mirror reflectivity level. Solar field mirrors are washed regularly, benefiting from the increased average reflectivity but adding to maintenance costs for the required labor, equipment, and demineralized water. KJC Operating Company (KJCOC) is constantly reevaluating the trade-offs among these factors and devising better mirror-washing techniques. A key to such evaluation, however, is knowledge of the average reflectivities before and after washing and the running level of reflectivity of the fields, especially during the summer peak period.

There are 546,624 mirror segments in the Kramer Junction, California, solar fields. Washing effectiveness varies according to mirror location (e.g., proximity to high-soiling areas, such as roads or cooling towers) and time of year. A large number of readings are required to gather the necessary information for rational decisions on washing frequency. The traditional method of taking readings-with a Devices and Services reflectometer-is time consuming and therefore expensive and ineffective.

Mirror soiling rates near the cooling towers are particularly high because of cooling tower drift. Extensive measurements are required to determine the true extent and rates involved. Furthermore, techniques to reduce soiling rates by treatment of the mirror surfaces could significantly reduce the washing problems in those locations.

This task addressed the practice at the Kramer Junction solar electric generating system (SEGS) plants with respect to methods and frequency of mirror washing, issues related to the assessment of the "average" reflectivity over a large collector field, and experiences relevant to mirror soiling in the Mojave desert.

\section{Experience on Mirror Reflectivity}

Many factors affect soiling rates of mirrors at the SEGS plants. The most critical factors include time of year, frequency of rainfall, proximity of mirrors to roads or other sources of airborne particulates, method and frequency of washing, and proximity to power plant equipment. At commercial plants such as the SEGS plants, the essential maintenance issue is straightforward: How often and how well should mirrors be washed to maximize net profits? The answer is found primarily by comparing the cost of washing against the gains in performance.

By the nature of the electricity power purchase agreement with the utility, the highest revenue gains are in summer, coinciding with the poorest conditions for mirror cleanliness, i.e., a dry, dusty environment. In winter, the electricity sales rates are lower, the solar field output is lower, and infrequent though periodic rains provide some natural washing. In general, the criterion at Kramer Junction is that the average mirror reflectivity be maintained in the $90 \%$ to $91 \%$ range, contrasted to $94 \%$ reflectivity for a new mirror. This value was arrived at by experience as a realistic, achievable, and cost-effective target. It is the current "rule of thumb" rather than the result of an in-depth cost/benefit analysis. 


\section{Measurement of Reflectivity}

For a number of years, reflectivity measurements at Kramer Junction were taken with a portable specular reflectometer built by Devices and Services. While accurate, it hindered rapid measurements of a large solar field because a source mirror is necessary to field calibrate the unit after each set (a single solar collector assembly [SCA]) of readings. Adjustment of the instrument on the mirror was slow, and log entry of the raw readings was done by hand. More recently, a new scatterometer " $\mu$ Scan" has been used to increase the cost-effectiveness of measuring solar field reflectivity. The unit can take a quick reading without careful adjustment, and the data are stored in local memory for later file transfer to a designated device. Accuracy of an individual reading is about $\pm 1 \%$. Because of these advantages, the number of data points per unit of time has increased by a factor of six, allowing for a broader survey of a solar field as a basis to calculate average reflectivity. The current method requires that four readings be taken on each of 20 SCAs to estimate the average reflectivity of the solar field of a $30-\mathrm{MW}$ plant (about $200,000 \mathrm{~m}^{2}$ ). While the specific SCAs are chosen at random, there is a pattern regarding the area of the solar field from which they are selected. That is, 4 SCAs are selected from the exterior zone near the edges of the field where degradation rates are higher, 8 SCAs are selected from the interior zone, and the final 8 SCAs are selected from the intermediate zone characterized by medium degradation. As of yet, no statistical analysis has been performed in an attempt to refine the sampling rate, though a recent Sandia National Laboratories document addresses a method to examine this issue.

\section{Soiling Rate}

Figure E-1 shows the results of a test on the degradation of mirror reflectivity with time for SEGS mirrors during the summer period-in this case on a test loop consisting of 16 SCAs. For purposes of this test, the mirrors in the test loop were not washed for three months. Reflectivity measurements were taken about twice per week with approximately 150 total readings taken each time. The value given is the reflectivity at 660 nanometers, which tends to correspond to the spectral reflectivity for mirrored glass. The drop-off is essentially linear, corresponding to a soiling rate of about 0.45 percentage points per day. This high rate does not usually occur during other periods of the year when airborne dust is less and natural washing is more frequent.

An exception to this rule, however, is the rare but real condition in which minimal rainfall occurs simultaneously with a high-dust or dirt level in the air. Such an event took place at Kramer Junction in late July 1995 when a light rain accompanied very high, gusty winds up to 35.8 meters per second ( 80 miles per hour). All of the solar fields were significantly affected, with average reflectivities dropping 12 to 19 percentage points to a level in the $72 \%$ to $79 \%$ range. Mechanical scrubbing with brushes was necessary to bring the mirrors back to a normal condition.

\footnotetext{
- Letter report from R. Prairie to M. Prairie dated November 25, 1995, on the statistical analysis of heliostat reflectivity data at Solar Two and a suggested sampling rate to determine mean cleanliness.
} 


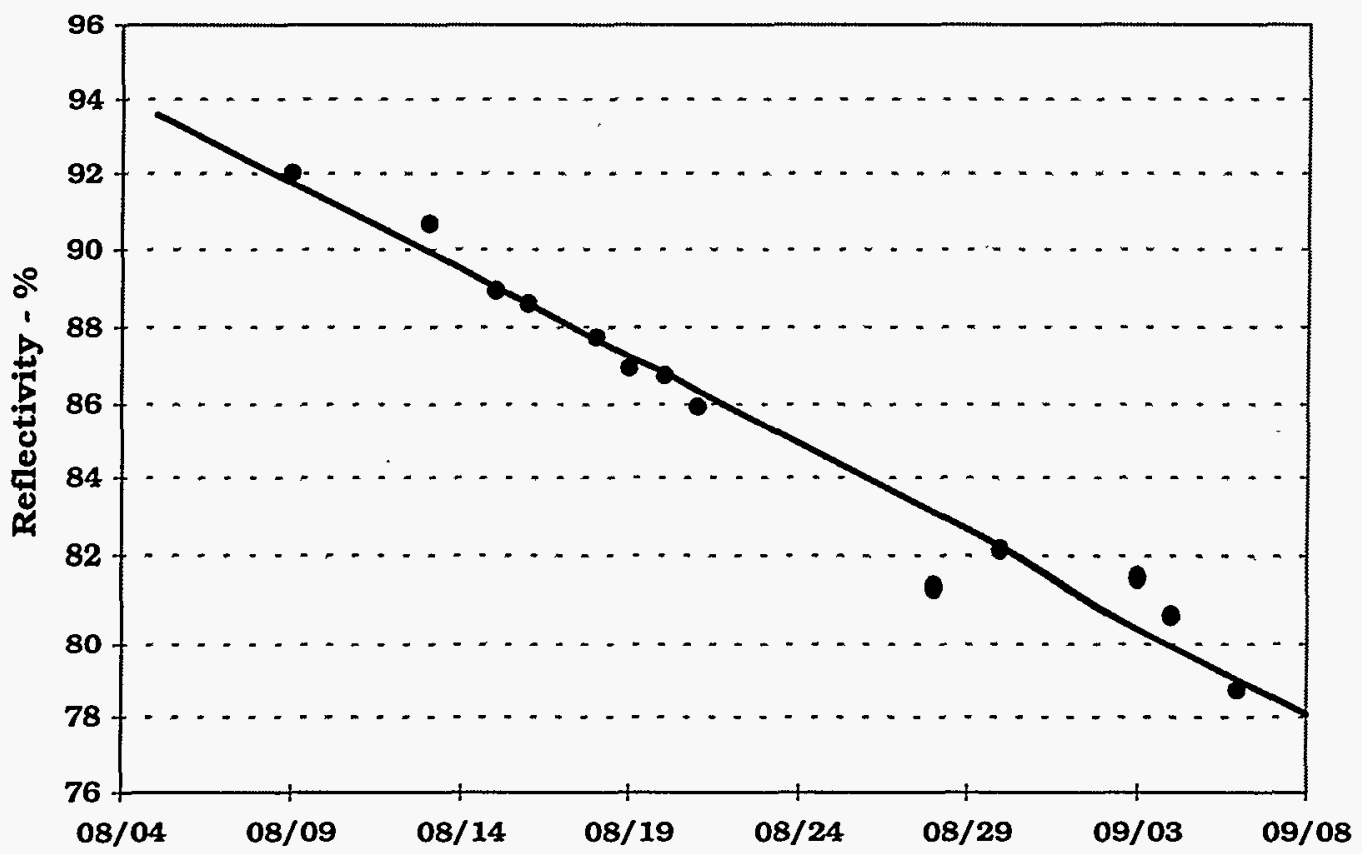

Figure E-1. Degradation of mirror reflectivity during summer months.

\section{Effect of Soiling on Thermal Output of the Solar Field}

Thermal output of the collector loop was measured in the mirror degradation test described above. The results were that a change in reflectivity of $1 \%$ resulted in a performance change of about $1.2 \%$ in solar field thermal delivery. This leverage effect occurs because solar field heat losses, which are dependent on operating temperature, remain constant while the radiation input to the receiver is directly proportional to the change in reflectivity. The revenues of the plant react proportionally. For the five Kramer Junction SEGS plants, a 1\% drop in reflectivity has an impact of approximately $\$ 185,000$ in annual revenues. This value would be much greater were it not for the hybrid design of these plants, which supplements the solar field with natural gas in the summer months when the revenues are highest.

\section{Other Effects on Soiling}

Soiling of mirrors is accelerated near roadways and the power block areas. Road effects have been reduced at the SEGS plants by paving the major roads between plants and within each plant. In addition, perimeter roads (although not paved) are treated annually with a soil stabilizer. Mechanical draft evaporative cooling towers discharge moisture, called drift, along with cooling air. This moisture combines with dust in the air and gently falls onto the mirrors in the proximity of the cooling tower, causing a much-higher-than-normal soiling rate. The extent and direction of the soiling depends on the amount of drift and the prevailing wind direction. Drift eliminators in the cooling tower can do much to reduce this problem. Figure E-2 illustrates the extent of the problem at the Kramer Junction plants by showing a typical pattern along with an indication of the level of degradation over a period of about one year. Note that the reflectivity can drop to near $40 \%$ in the worst SCAs. In these $30-\mathrm{MW}$ plants, approximately $2 \%$ to $3 \%$ of the solar field is affected by cooling tower drift. 


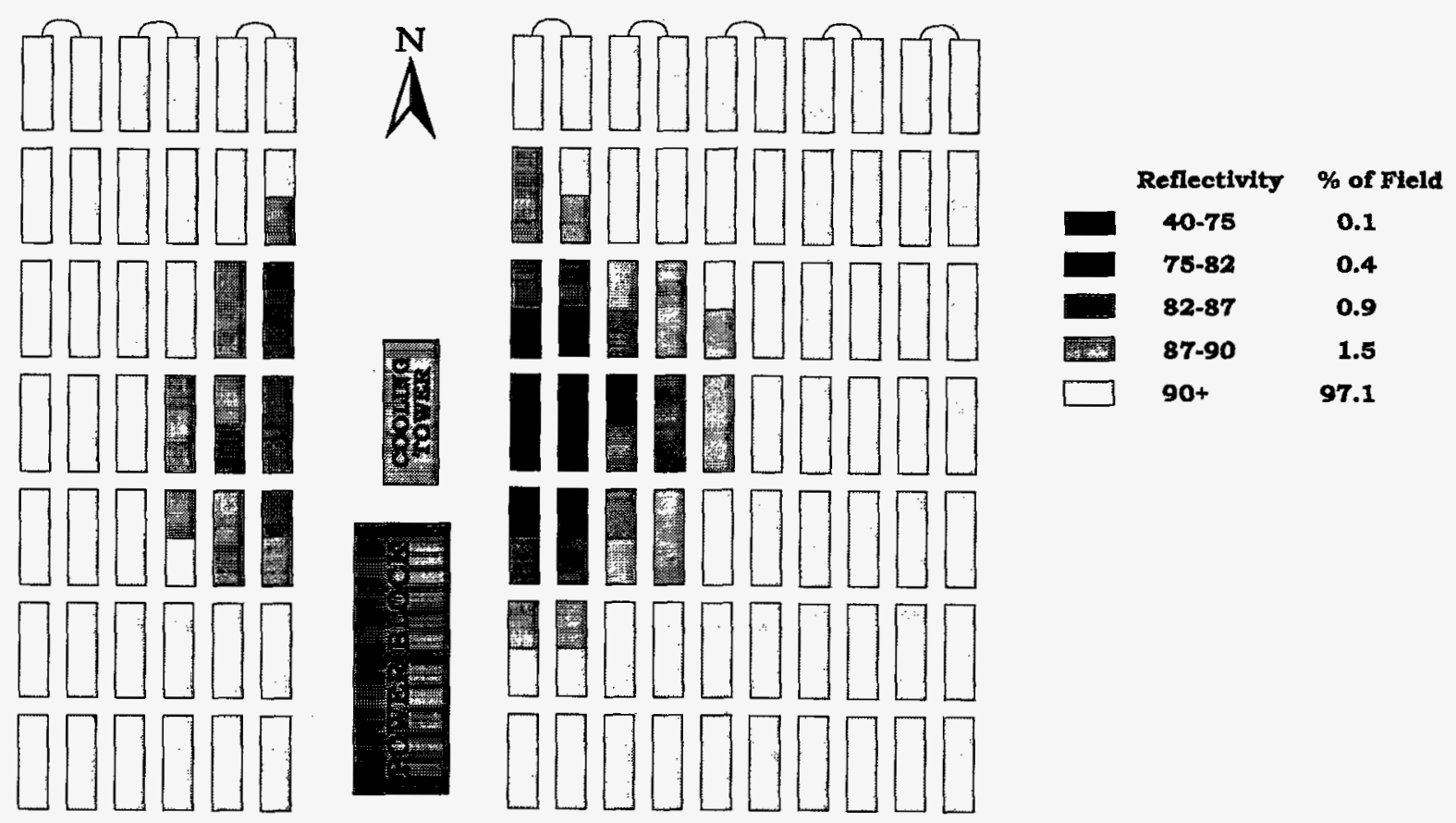

Figure E-2. Influence of cooling tower drift on mirror soiling.

\section{Mirror-Washing Methods}

\section{Routine Cleaning}

Three methods are used for mirror washing at the Kramer Junction SEGS plants. For all methods, the wash crew uses the solar field control system to place adjacent rows of collectors in a position where the two rows face each other, much like this: ( ). Demineralized water is always used. Figures E-3 and E-4 show pictures of the methods used before the Operation and Maintenance Improvement Program (O\&MIP) began. If rain or snow is likely, the SCAs are tilted upward to take full advantage of the weather. Snow provides a scrubbing effect that is unparalleled by any of the maintenance methods, invariably bringing all the solar fields up to a like-new reflectivity of about $94.7 \%$.

The high-pressure rig (Figure E-3) consists of a tractor-pulled trailer that contains a water tank, positive displacement water pumps that deliver water at 207 bars (3,000 psi), and two spray nozzles. A three-person crew is used: one person to drive the truck and the others to spray the mirrors using the hand-held nozzles. The rig moves down one row and returns cleaning the adjacent row.

The high-volume method (Figure E-4) uses a large-capacity water truck driven by a single driver and employs fixed nozzles on each side of the truck to spray the rows of mirrors simultaneously with a "deluge-type" stream of water. Various nozzle designs have been tested to arrive at the most effective design. The truck holds $15.1 \mathrm{~m}^{3}$ (4,000 gallons) of demineralized water. On-off control of the nozzles is accomplished from the cab. 


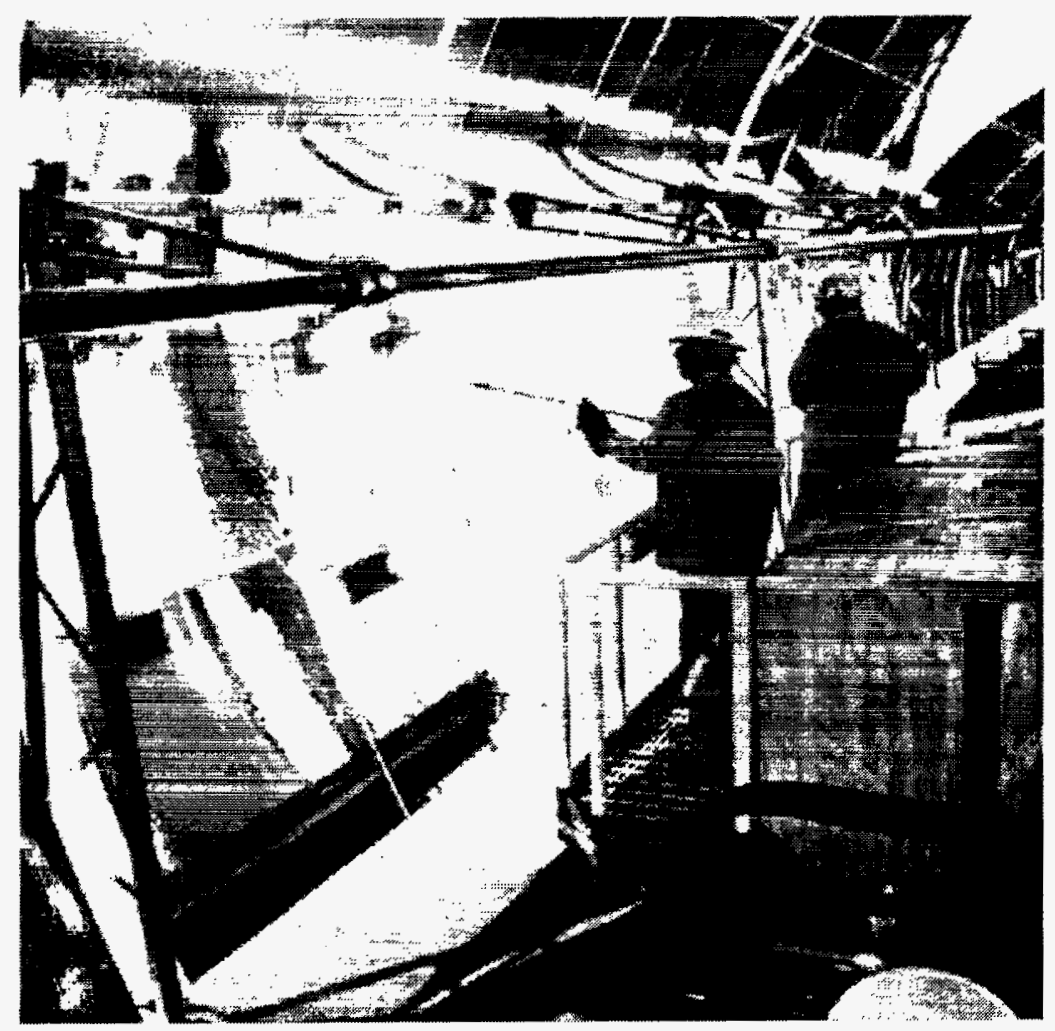

Figure E-3. Illustration of high-pressure cleaning.

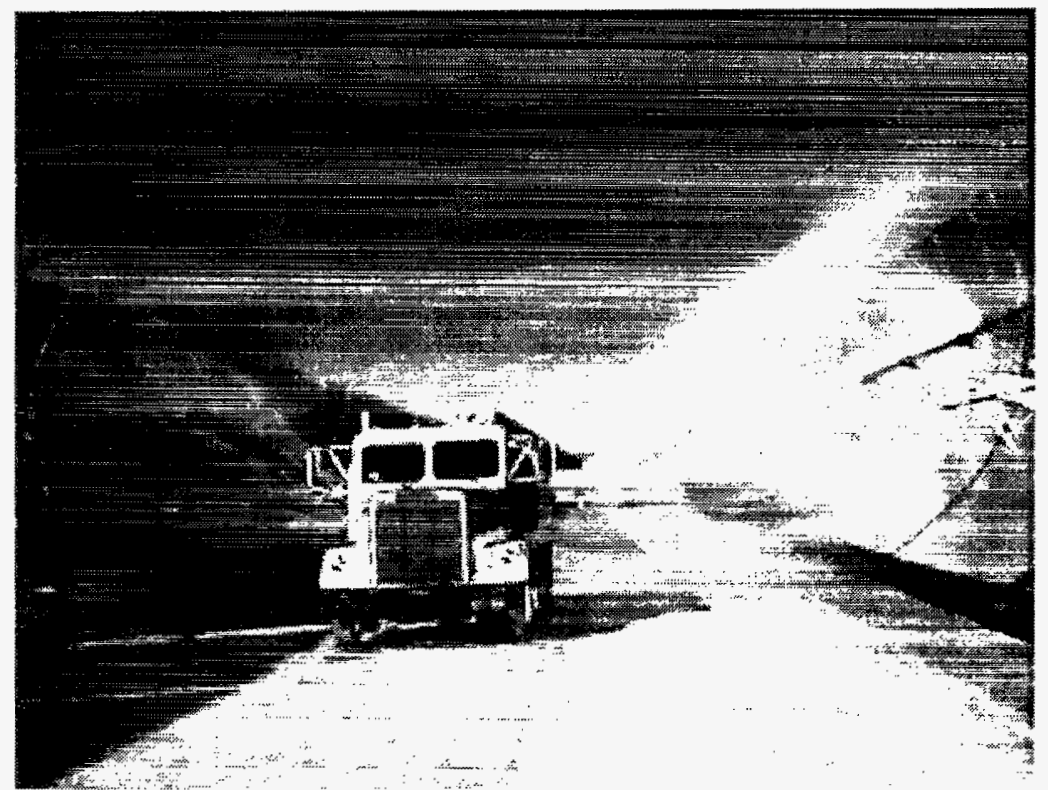

Figure E-4. Illustration of deluge cleaning. 
The deluge method uses 55 gallons of water per LS-2 SCA (approximately 0.23 gallons per square meter of aperture area) while the high-pressure spray method uses 45 gallons of water per LS-2 SCA (approximately 0.19 gallons per square meter of aperture area). Though the deluge method uses $20 \%$ more water per unit area of mirror than the high-pressure spray method, it is four times as fast, and the work-hours per collector are consequently less by a factor of 12 . The high-pressure spray will raise the reflectivity about 3 percentage points, while the deluge spraying will result in a 1 percentage point increase. As illustrated in Figure E-5, a typical pattern is to alternate a high pressure spray with two deluge washings, usually attempting to maintain an average mirror reflectivity of about $90 \%$. Note that the high-pressure spray cleaning is done on a three-week cycle with two weekly deluge washes in between. This aggressive washing schedule takes place only during the summer on-peak period. Average cost of the highpressure spray method is about $\$ 0.66$ per square meter per year, compared to $\$ 0.14$ for the deluge spray method. The two methods cost $\$ 0.013$ and $\$ 0.003$ per square meter per wash, respectively.

Mirror washing took another step forward at Kramer Junction during the O\&MIP with the development and use of the new "Mr. Twister" mirror-washing rig, which reduces work force needs and improves the quality of washing. The Mr. Twister rig (see Figure E-6) consists of a tractor pulling a wheeled tank-and-pump unit. A single person drives the rig and controls the washing from the cab.

The tractor is a Ford Model 4430 specialty vehicle, characterized by its small frame and high horsepower to pull the 25,000-pound water/pump unit. The tank holds 1,500 gallons of demineralized water, which is delivered by a positive displacement General pump driven by a John Deere 150-hp diesel engine. The pump can supply up to 45 gallons per minute at a pressure of 3,500 psi.

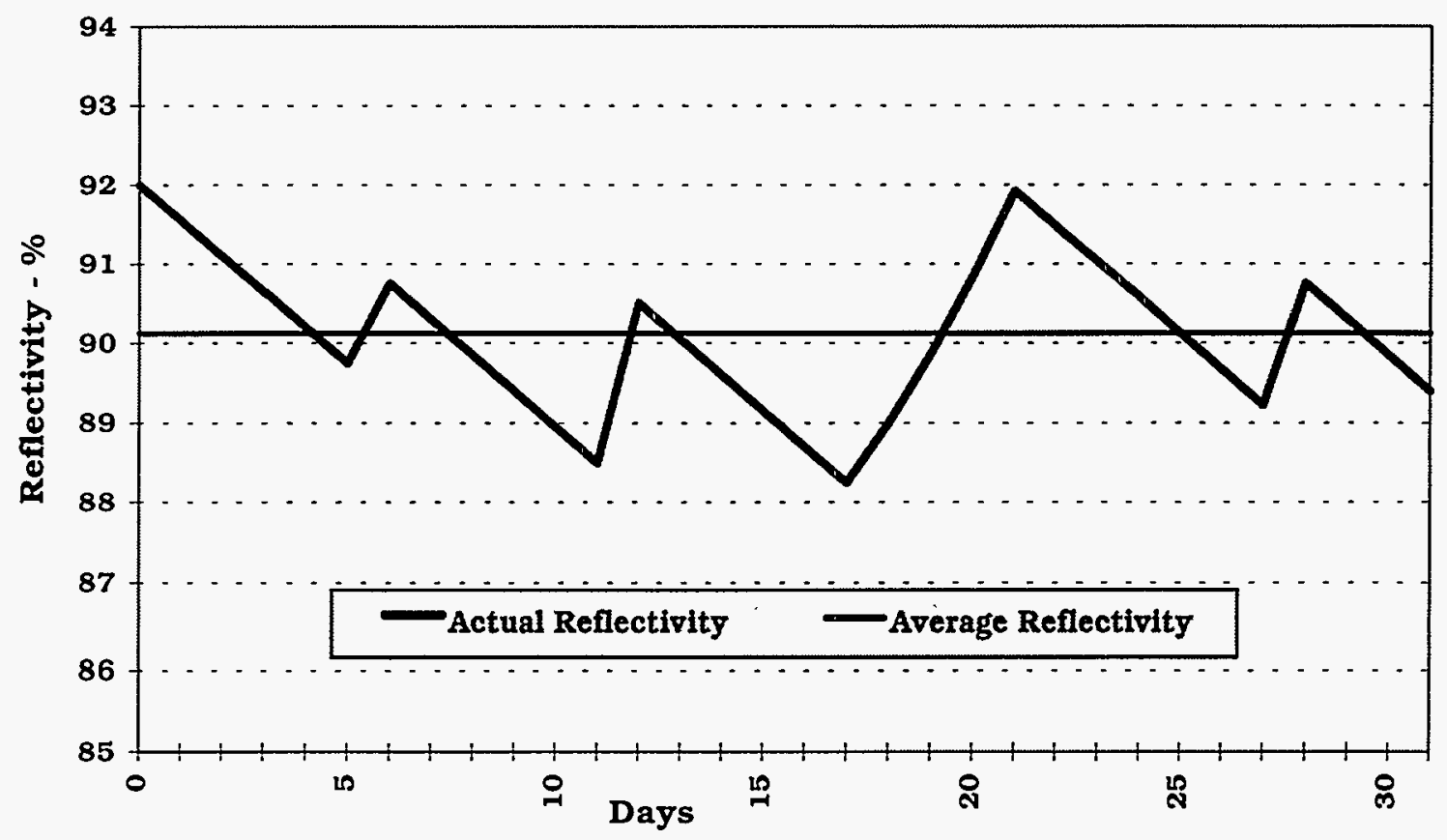

Figure E-5. Typical wash cycle for SEGS mirrors. 


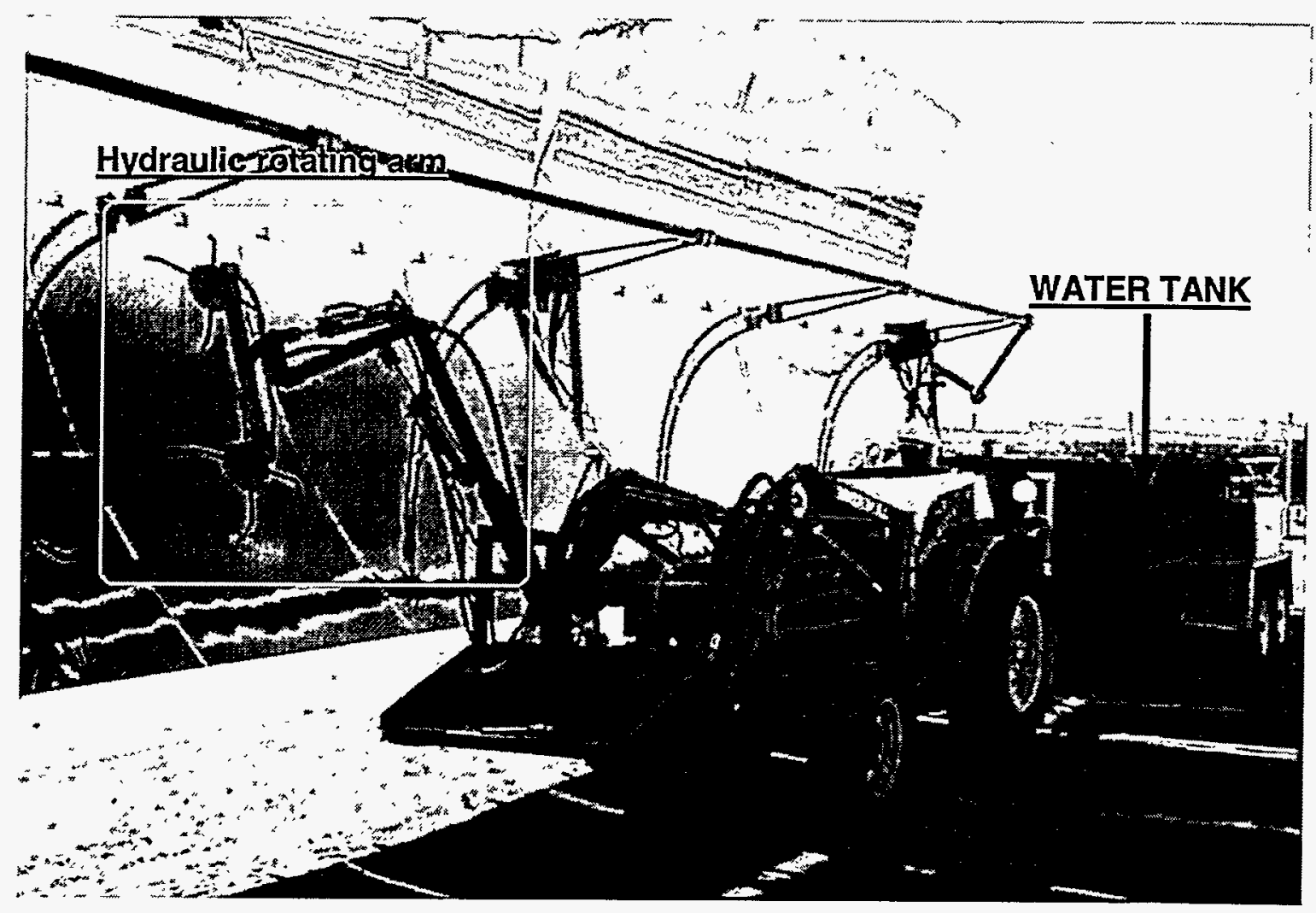

Figure E-6. "Mr. Twister."

The tractor is mounted with a controllable arm mounted in the front. The arm, with fivemovement articulated control from within the tractor cab, supports a configuration of spray arms that are fed by high-pressure water from the tank unit. The current configuration supports two swivel assemblies, each with four diffuser arms. Each arm contains a \#6 carbide nozzle (0.062-inch inside diameter) fed by a forcing cone with flow straightener to minimize spiraling in the water stream leaving the nozzle.

Many different variables affecting cleaning effectiveness are possible and have been evaluated in this program:

- Design of rotating arm and number of nozzles

- Nozzle type and dimensions

- Water pressure

- Water flow (determined by the above parameters)

- Standoff distance

- Rotation speed

- Frequency of cleaning 
After a series of tests, which are continuing, the present conclusion is that the following arrangement provides maximum effectiveness:

- Water pressure at $3000 \mathrm{psi}$

- Double rotating arm with two sets of swivel assemblies with four nozzles, able to spray both top and bottom halves of an SCA at the same time

- Arm articulated to a position within a foot of the HCE in order to be as close to the mirror as possible

With one person driving and controlling the rig, it takes only three nights to fully wash the solar field of a 30-MW plant, achieving an average reflectivity of more than $92 \%$. Water use is about the same as with the previous high-pressure method, i.e., less than 45 gallons per SCA. Glass breakage, never a large problem, has been further reduced in comparison to previous methods.

The sources of water are the demineralizing systems at each plant. To speed the mirror-washing process, a "nursing" tank truck containing 4,000 gallons of demineralized water is filled and driven to the location of the Mr. Twister. This takes half of one person's time. This truck can fill the 1,500-gallon Mr. Twister tank in less than five minutes. After three fills, the nurse vehicle returns to the demineralizer unit, is refilled, and returns to the field.

The development of the Mr. Twister is the latest manifestation of the evolution of mirrorcleaning tools at Kramer Junction. The Mr. Twister is replacing the three-person high-pressure rig. Both provide "deep" cleaning of the mirrors to reflectivities in the $93 \%$ to $94 \%$ range. However, operation of the Mr. Twister requires one and one-half persons rather than three and permits complete cleaning of a 30-MW solar field in three nights instead of four. The deluge truck complements the deep cleaning with alternate washings, which are faster, but less effective.

\section{Special Cleaning}

Over the years, there has been a degradation of the reflectivity on a narrow band of about 6 to 12 inches on the lower edge of the mirrors. Consequently, more rigorous cleaning is required every two to three years. This cleaning consists of hand cleaning with demineralized water and a hard brush.

Similarly, a labor-intensive method is required to clean heavily soiled mirrors near the cooling towers. At the Kramer Junction plants, a very dilute solution of $3 \%$ hydrofluoric acid wash was recently applied on a one-time basis. The process consisted of the application of an acid gel in mist form, which was allowed to cling to the mirrors for about 10 minutes. A neutralizer was then applied, followed by high-pressure spraying with demineralized water. Cleaning took about 45 minutes per SCA. The results generally showed a final reflectivity averaging $88 \%$ starting from initial reflectivities as low as $40 \%$, with some exceptions ranging from $10 \%$ to $15 \%$. Because of the acid use, albeit dilute, this method is not recommended on a regular basis and will not be used again at Kramer Junction. Rather, the mirrors near the cooling towers will be closely monitored, and periodic scrubbing will be used with water only or a more benign cleaning solution. 


\section{Appendix F \\ TORQUE TUBE ALIGNMENT}

\section{Background}

The thermal performance of the solar field in any solar thermal electric plant is strongly influenced by the ability of the collectors to focus on the receiver. This focus requires excellent alignment between the tracking system, the collector structure, and the reflective surface. In the solar electric generating system (SEGS) collectors, this translates to alignment of the sun sensor, torque tube, and mirror supporting arms. In a parabolic trough plant, the receiver (heat collection element) is also attached to the structure, and thus, the alignment of this component is another critical factor.

SEGS IV was constructed in 1986. The LS-2 collectors in SEGS IV were designed for a high optical accuracy, but realization of the design specifications depends on proper fabrication and installation. After installation, alignment of the collector components will deteriorate over time, at some unknown rate because of normal movement and the effects of expected structural forces (e.g., gravity, wind, and sonic booms).

Observations of the focusing accuracy and measurements on the performance of the solar field indicated an unacceptable deterioration in alignment. It was not known, however, to what extent realignment in the field would improve this situation and bring the optical accuracy back to design specifications. A decision was made to conduct an extensive realignment of the torque tubes in SEGS IV as an important and initial step in the evaluation and implementation of field realignment techniques.

The scope of this task was to develop and conduct new alignment procedures on the torque tubes in the SEGS IV solar field, measuring alignment deviations between adjacent torque tubes before and after the procedure.

\section{Objectives}

The objectives were to take random but statistically significant measurements of the torque tube alignment in SEGS IV, take corrective actions for realignment of the torque tube, and quantify the new alignment. There are six torque tubes in an LS-2 collector, designated $1 \mathrm{~S}, 2 \mathrm{~S}, 3 \mathrm{~S}, 1 \mathrm{~N}$, $2 \mathrm{~N}$, and $3 \mathrm{~N}$. The $\mathrm{N}$ and $\mathrm{S}$ designate the north and south directions relative to the drive pylon, while the numbers designate the position from the drive pylon ( 1 being closest). In a different procedure, the sun sensor and the $1 \mathrm{~S}$ torque tube are aligned for tracking accuracy. The objective of the torque tube alignment is to align all the torque tubes as closely as possible to the is tube.

The plan of action consisted of the following steps:

1. Welding the torque transfer to the axle of the gear to eliminate the backlash.

2. Before alignment, measuring and recording the position of each torque tube relative to the reference points (in the first moment of transfer). 
3. Calculating the deviation of each adjacent torque tube and determining the correction needed.

4. Conducting realignment.

5. Conducting a quality inspection and analyzing the results.

The measurement of the torque tube alignments in a solar collector assembly (SCA) is conducted by attaching a customized alignment device to each torque tube and measuring the angular deviation of tube relative to the $1 \mathrm{~S}$ tube; this is the absolute deviation. This measurement is done with the collector in the $90^{\circ}$ position. Using this measurement, a calculation is made to determine how far the tube must be rotated to achieve alignment, and this is marked on the tube. Following this step, a separate work crew conducts the necessary adjustments.

Once the realignment adjustments are made, the measurement crew remeasures the alignment of the torque tubes in the SCA. The improvement in alignment is evaluated by noting the deviations before and after the realignment procedure.

The anticipated benefit was an improvement of the alignment and, consequently, the focusing accuracy of the LS-2 collectors in SEGS IV. Earlier test results on LS-3 collectors suggest that alignment of the primary support structure may have a $5 \%$ to $10 \%$ impact on thermal efficiency of the collector, which would have a major impact on plant performance.

\section{Results}

The results in the SEGS IV solar field obtained by realignment of the torque tubes has been evaluated by taking measurements on selected SCAs in every quadrant. While "before" measurements are taken on each SCA as part of the alignment procedure, the "after" measurements were taken on about $12 \%$ of the torque tubes to check the effectiveness of the procedure.

The results are presented as standard deviations as a function of torque location in the SCA for different quadrants of the solar field (note that, by definition, the deviation of the $1 \mathrm{~S}$ torque tube is zero). Figure F-1 graphically illustrates the standard deviations before and after alignment. An ideal alignment, as represented on such a graph, would be a horizontal band at the zero position. The results indicate that the southeast quadrant had the poorest alignment before the procedure and, while improved considerably, still represents the poorest alignment. 


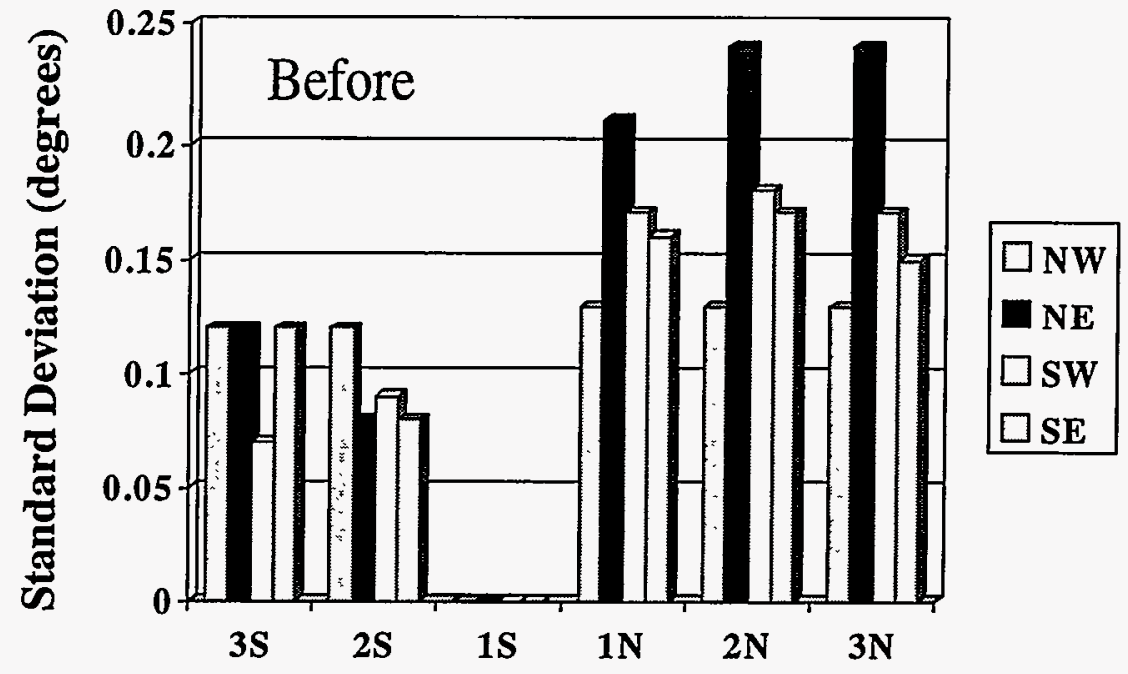

Torque Tube (location in the SCA)

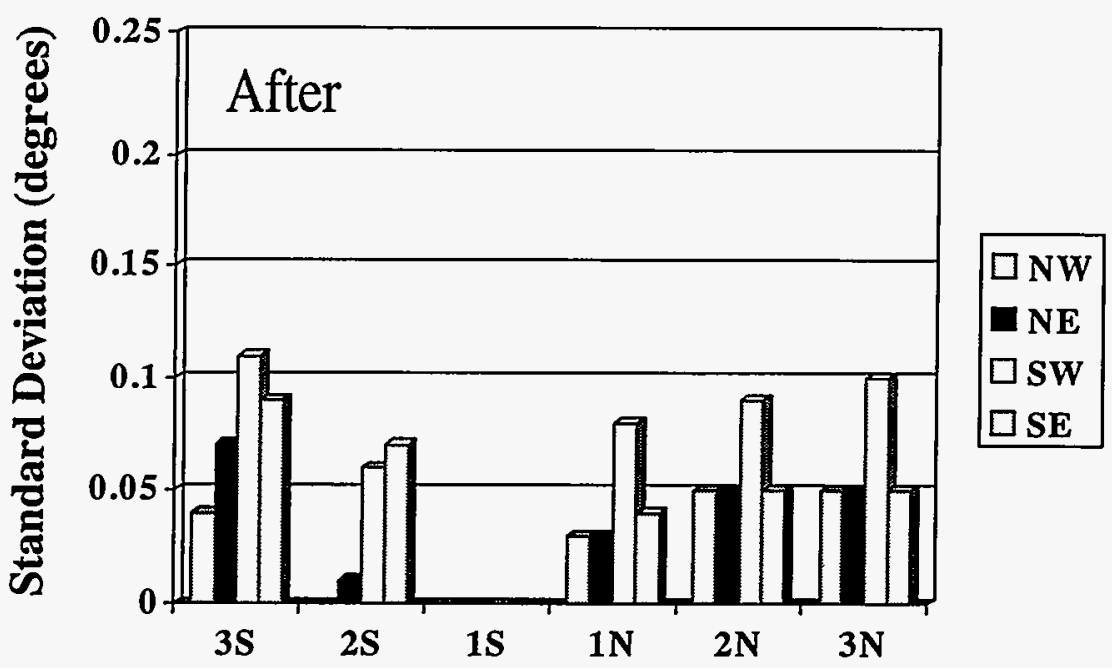

Torque Tube (location in the SCA)

Figure F-1. Torque tube alignment of SEGS IV before (top) and after (bottom) realignment. 
This page intentionally left blank. 


\section{Appendix G \\ LS-3 Collector Loop Testing}

\section{Background}

LS-3 collectors were first installed at Solar Electric Generating System (SEGS) VII, constituting approximately one-half of the solar field (184 solar collector assemblies [SCAs]). During the initial years of operation, it was clear that these LS-3 collectors were performing well below design efficiency. ${ }^{*}$ In-depth analysis of the problems focused attention in the areas discussed below.

- General alignment of collector components (truss, sun sensor, heat collection elements [HCEs]). The truss design of the LS-3 collector requires careful alignment of all components during construction. Inadequate construction practice and quality control at installation necessitated extensive post-construction realignment.

- Center of gravity. In SEGS VII, the metal used for the truss was heavier than specifications, shifting the center of gravity away from the HCE tube closer to the mirrors. To correct this problem, counterweights are required to shift the center of gravity back to its design position.

- Excess friction in the bearings. Friction in the SCA bearings exceeded design, causing a twisting effect on the trusses (each SCA is made of eight trusses and eight bearings). Special corrective alignment is required to compensate for this twisting effect.

- Tracking error. A tracking error occurs because of a slight backward rotation of the SCAs after each forward tracking increment, apparently because of play in the gearbox. As a test, a brake has been installed on the gear shaft of one SCA to stop this backward rotation; however, more testing is necessary to find the best solution.

Correction of these problems can be costly, and data were needed on the effectiveness and benefits of possible solutions in order to devise the most cost-effective operation and maintenance strategy for the SEGS VII solar field. Consequently, two phases of in situ field tests were conducted to quantify the impact of these problems and to measure the effectiveness of corrective actions. This task was conducted after previous corrective actions had been conducted on the solar field, except for the two loops that were left unaltered for future testing. In this way, the effect of each modification can be better understood and quantified.

\section{Phase 1 - Test Description}

The two unmodified test loops were instrumented for testing. The loops are located in the northeast quadrant of SEGS VII, at rows 31 through 34. One loop was left unmodified, according to the initial installation (reference loop); the other loop (test loop) was to be modified and

\footnotetext{
* Most of these problems were corrected in the design and installation of the LS-3 SCAs used in SEGS VIII and IX; furthermore, these problems were specific to the LS-3s at Kramer Junction, and not to the LS-2s that constitute most of the solar fields at that site. Testing was required at this time to understand the relative importance of each improvement.
} 
compared to the unmodified reference loop. Figure G-1 presents a simple schematic of the test loop configuration.

Each loop consists of eight LS-3 SCAs. Turbine flow meters were placed on the inlet of each loop, with high-precision RTDs for temperature measurement at the inlet and outlet. All instrumentation was tied into a centrally located data logger. Data were measured every second and recorded as two-minute averages. Before each day of testing, both loops had a complete mirror wash to maintain an equivalent reflectivity on each loop. Broken glass HCE tubes, lost vacuum HCE tubes, and missing mirrors were initially noted and kept at equivalent levels for the duration of the testing. This test was designed to be a comparison test, primarily seeking to evaluate the percentage improvement because of correction of the modifications discussed below.

The first objective was to establish a comparison of both loops as originally installed or unmodified. The second objective was to modify the test loop in a series of incremental steps dealing with counterweights, sun sensor alignment, truss alignment, and HCE alignment. In this way, the relative effect of each modification could be carefully measured.

Measurement of the performance improvements provides the information necessary to make cost/benefit trade-offs on solar field maintenance on the Kramer Junction LS-3 solar collector assemblies. The cost of each modification is based on the number of workers, skill level, and time required for the procedure. The test results give the basis to estimate the expected improvement and revenue increase from each modification. While these results are specific to the condition of the LS-3s in SEGS VII, the insight gained here contributes toward a more general understanding of the benefits that might be anticipated from periodic alignments in similar solar fields.

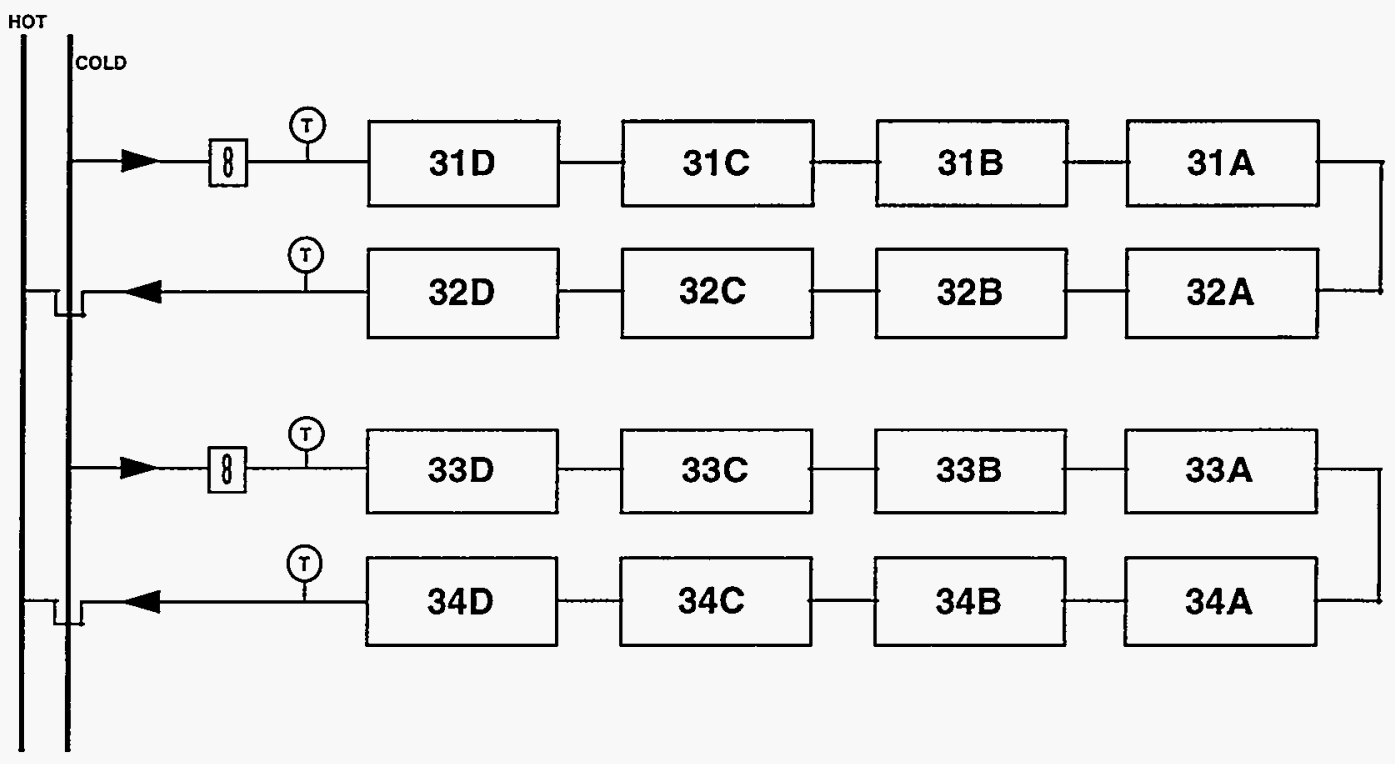

Figure G-1. SEGS VII test loop schematic. 


\section{Summary Test Results}

The first set of data, shown in Figure G-2, is a comparison of both loops as originally installed or unmodified. It can be noted that the performance of both test loops is quite similar and that both are significantly below design level.

The test loop was then modified in a series of incremental steps to measure the effect of adjustments to the counterweights, sun sensor alignment, truss alignment, and HCE alignment.

Summary results of the combined effects of the modifications compared to the reference loop are shown in Figure G-3.

Figure G-2 verifies that the performance of each loop was equivalent at the beginning of the test. Each LS-3 collector contains $24 \mathrm{HCE}$ tubes and 224 mirrors. At the start of testing, the reference loop had 0 broken glass HCE tubes, 8 lost vacuum tubes, and 0 missing mirrors, while the test loop had 0 broken glass HCE tubes, 6 lost vacuum tubes, and 4 missing mirrors. Performance of the unmodified collectors peaks in the late morning and steadily decreases during the remainder of the day. This performance can largely be attributed to the interaction between the effects of the off-design center of gravity and the excessive bearing friction, which tend to cancel each other in the morning but are additive in the afternoon.

After the modifications, however, the daily performance of the test loop, as shown in Figure G-3, improved by $39.5 \%$. This improvement, while significant, still leaves the performance at about 6 percentage points below design level. Several factors contribute to this continuing shortfall. One factor is the integrity of the components-at this stage of the test, the reference loop had 1 broken glass HCE tube, 9 lost vacuum tubes, and 6 missing mirrors, while the test loop had 1 broken glass HCE tube, 8 lost vacuum tubes, and 9 missing mirrors. Another factor is the tracking problem, which is indicated in the plot by the jagged lines and is most prominent in the afternoon. Additional factors include soiling of the HCE envelope glass, inadequate treatment of HCE shadowing in the previous design model, and incomplete realignment of the HCEs (realignment was conducted only on the vertical axis while at the solar noon position). The primary objectives of the present testing, however, were to measure the effects of the modifications; a future test will be devoted to a more careful comparison to design performance.

\section{Incremental Effects of Modifications}

The changes and adjustments to the test loop used for modifications were conducted incrementally in the following order:

- Addition of counterweights

- Alignment of sun sensor

- Alignment of truss structure

- Alignment of HCEs 


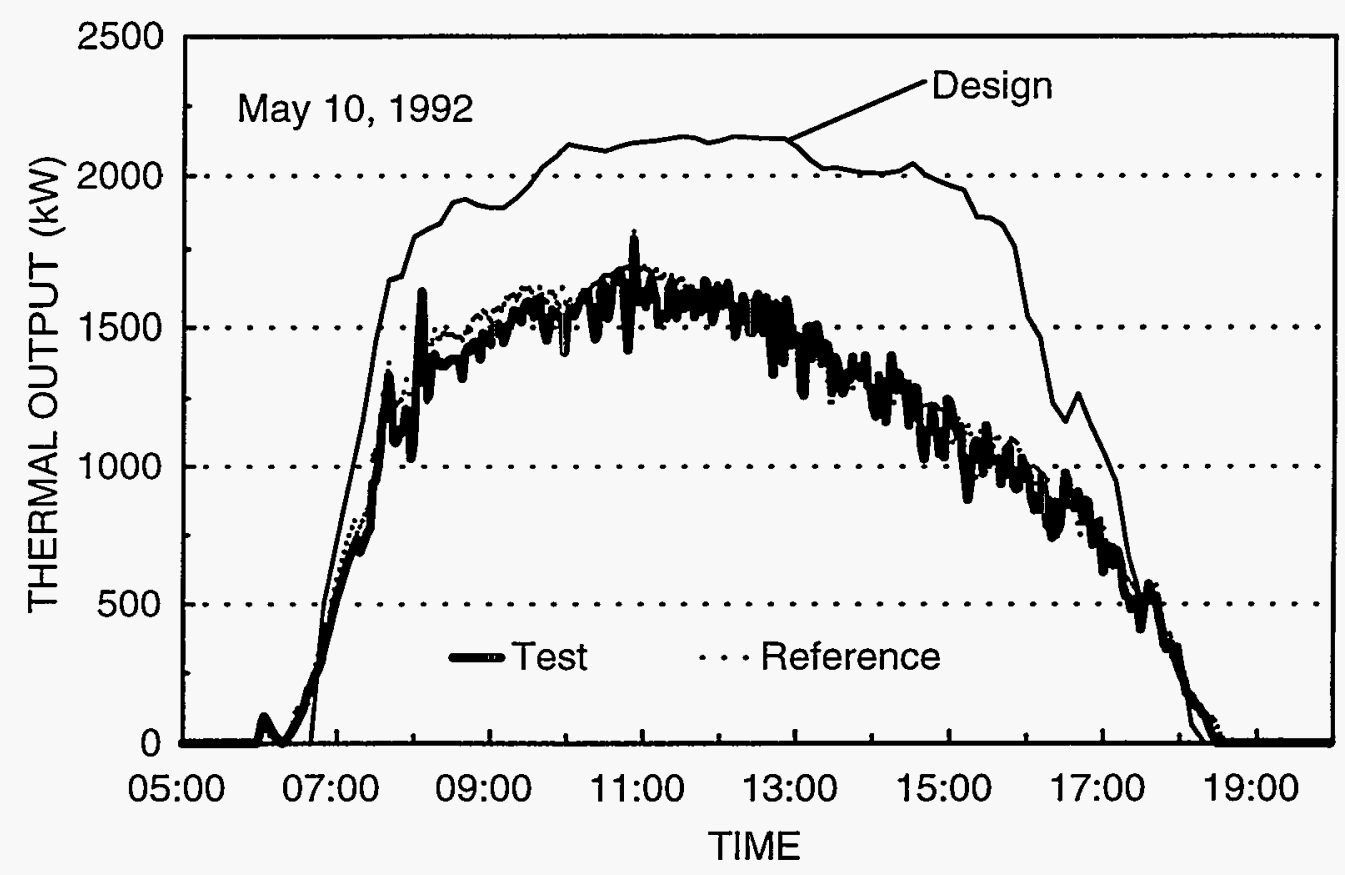

Figure G-2. Comparison of test loop performance before modifications.

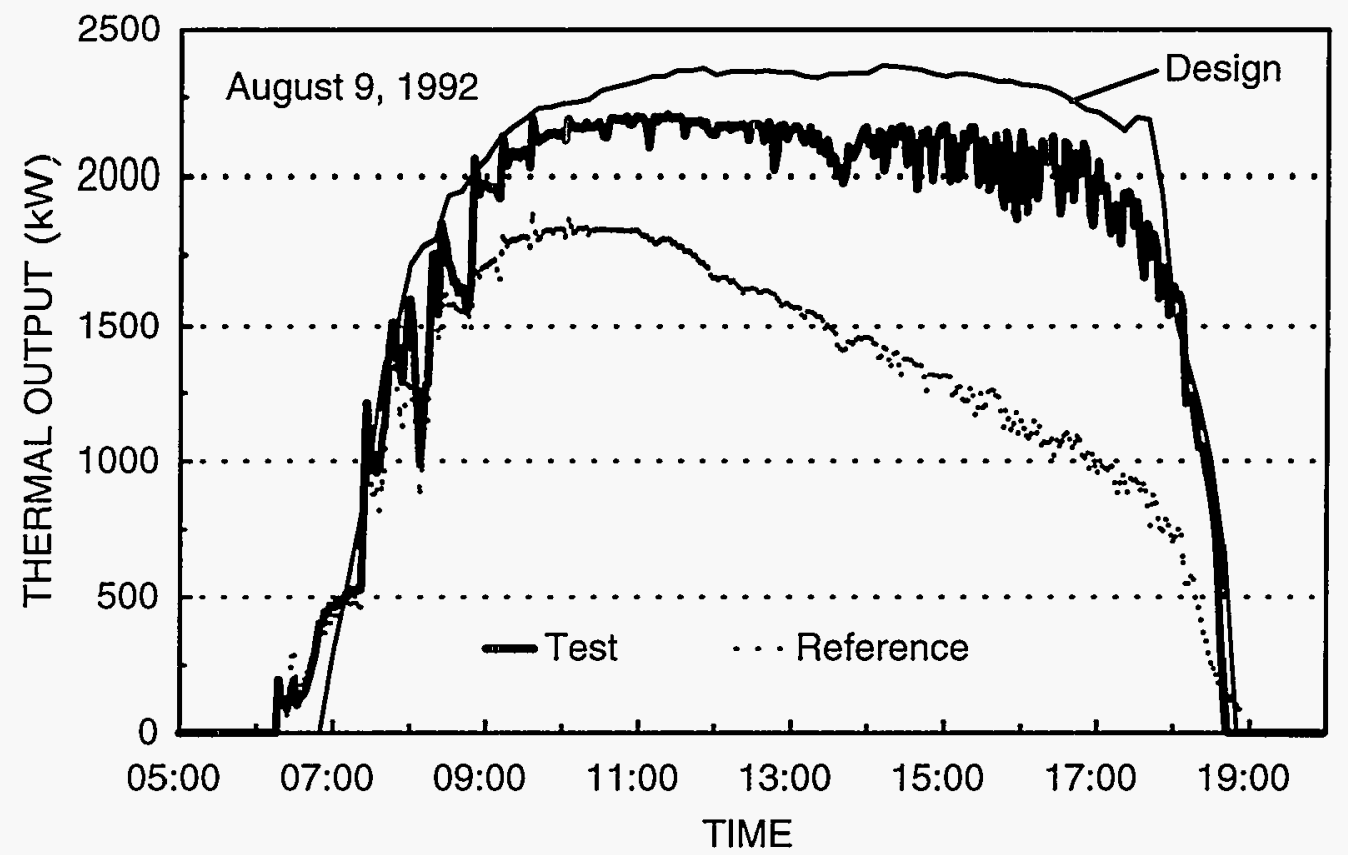

Figure G-3. Comparison of test loop performance after modifications.

The impacts of the modifications, then, were not measured independently (i.e., the impact of each modification on an unmodified SCA), and there is no assurance that the relative impact of each change would have been the same if conducted in a different order. Nevertheless, it is believed that the indications of relative improvement are reasonably accurate, and the result following all modifications would be identical with any order of the individual changes. 
Figures G-4 through G-7 show the sequence of impacts of each modification on the instantaneous thermal delivery of test loop. Figure G-8, on the other hand, presents the results in a different format, showing the energy delivery of the modified test loop relative to the reference or unmodified test loop after each incremental modification was made. The effect of any single modification is influenced by the previous modifications; thus, it is the result (top curve) that has the most significance. In all cases, the improvement ratio rises in the afternoon because of the poor performance of the unmodified test loop during those hours.

A final check on the results of this task consisted of moving the accurate flow and temperature instrumentation to an arbitrary loop in the solar field to compare the performance of a modified test loop to a typical solar field loop that had previously been realigned and adjusted. The comparison of thermal delivery provides some measure of the effectiveness of the previous work conducted on the LS-3 collectors in SEGS VII. Data were collected for this purpose in late September and early October 1993. While further evaluation is required, the tentative conclusion is that a recent test loop may out-perform the "typical" loop by a few percentage points.

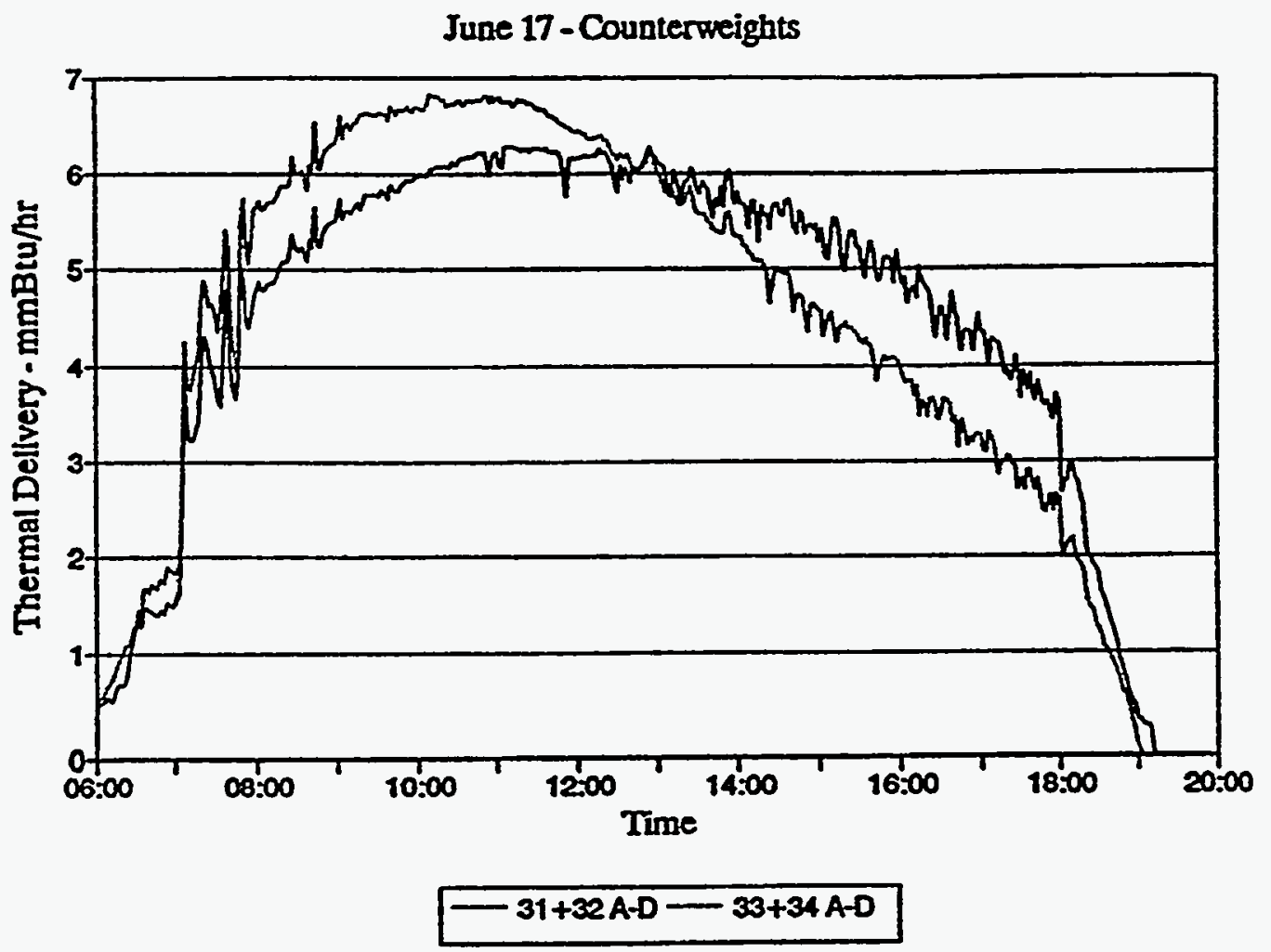

Figure G-4. Effect of counterweights on test rows 33 and 34. 
July 2 - Sun Sensors, CW

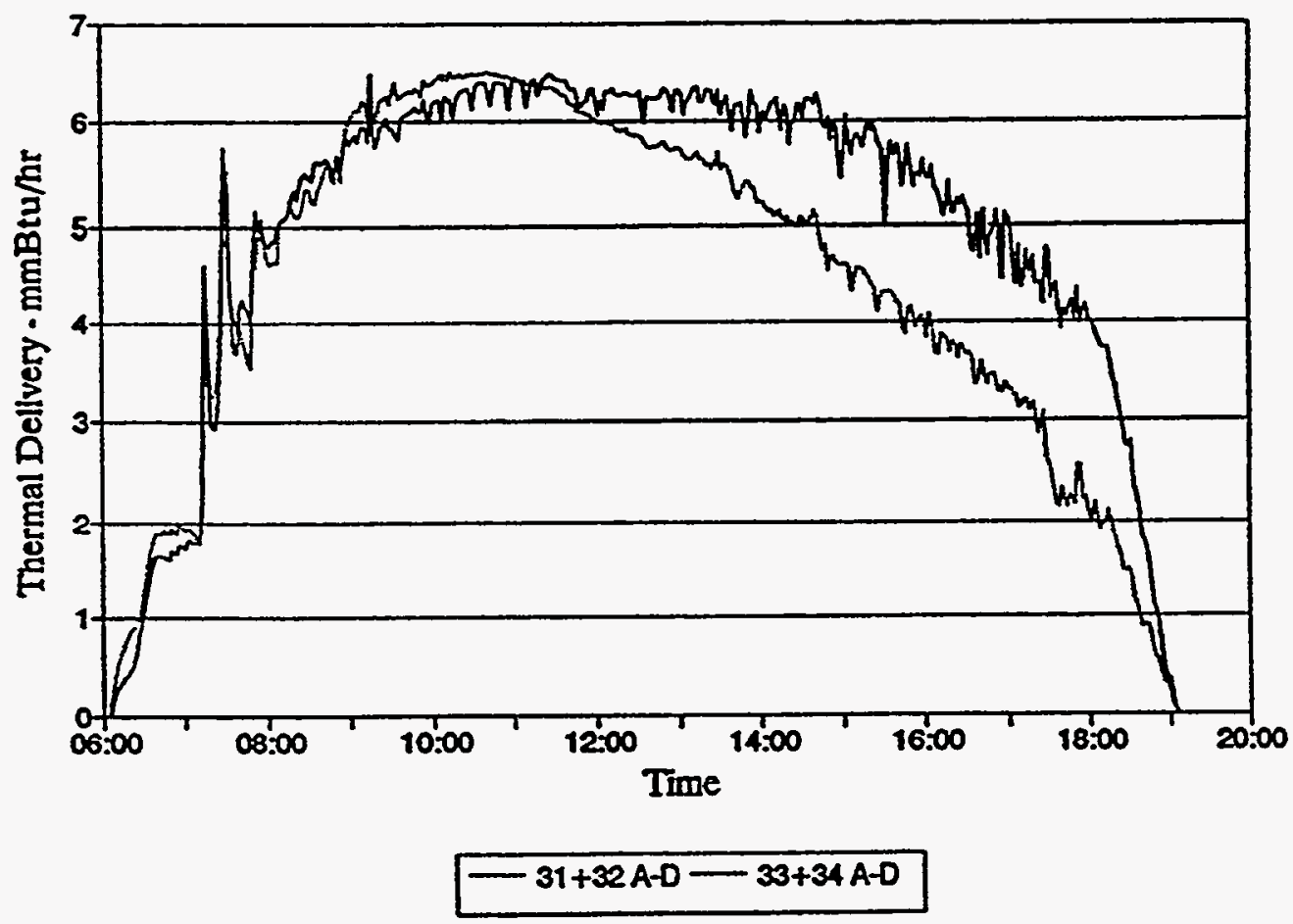

Figure G-5. Addition of sun sensor alignment to test rows 33 and 34 .

July 23 - Truss Alignment, SS, CW

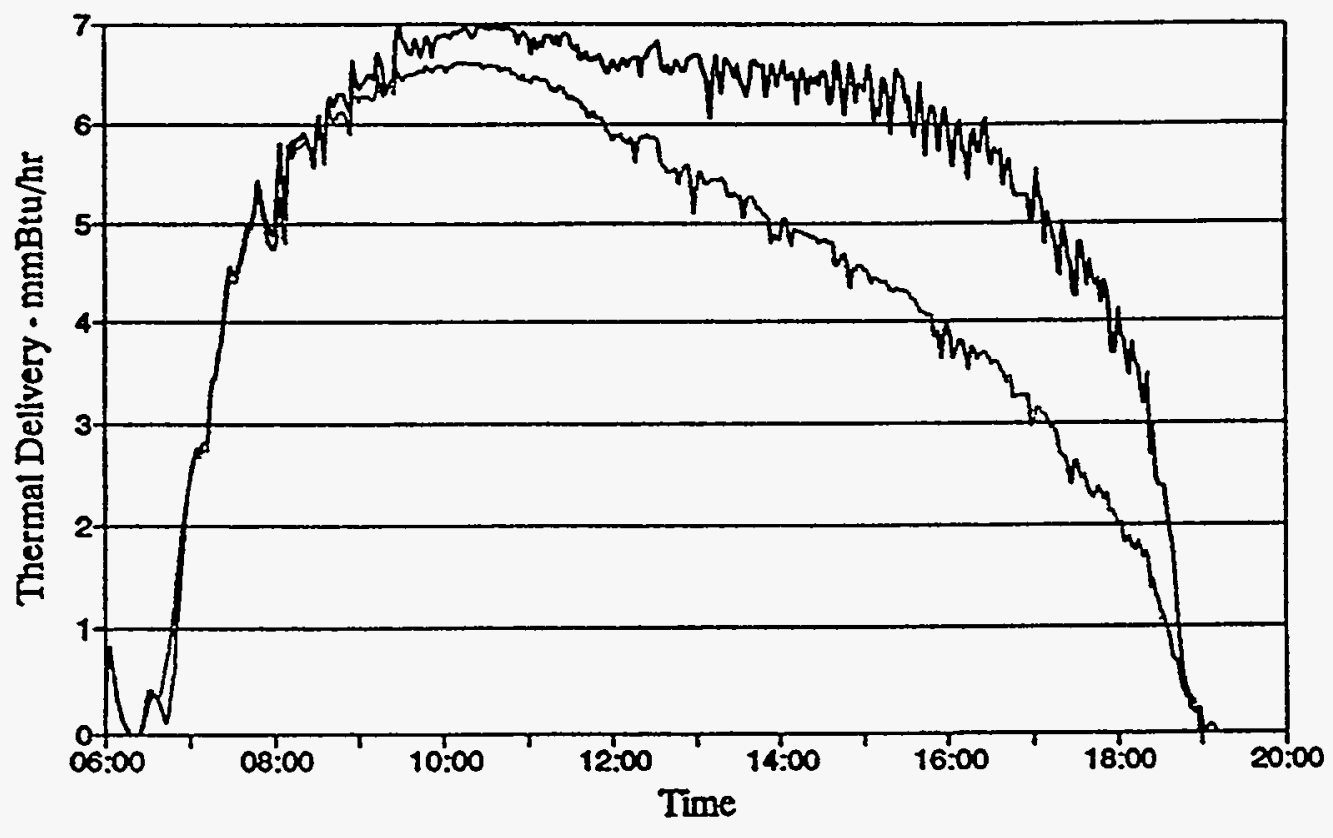

$-31+32 A-D-33+34 A-D$

Figure G-6. Addition of truss alignment to test rows 33 and 34 . 


\section{Aug 9 - HCE Alignment, TA, SS, CW}

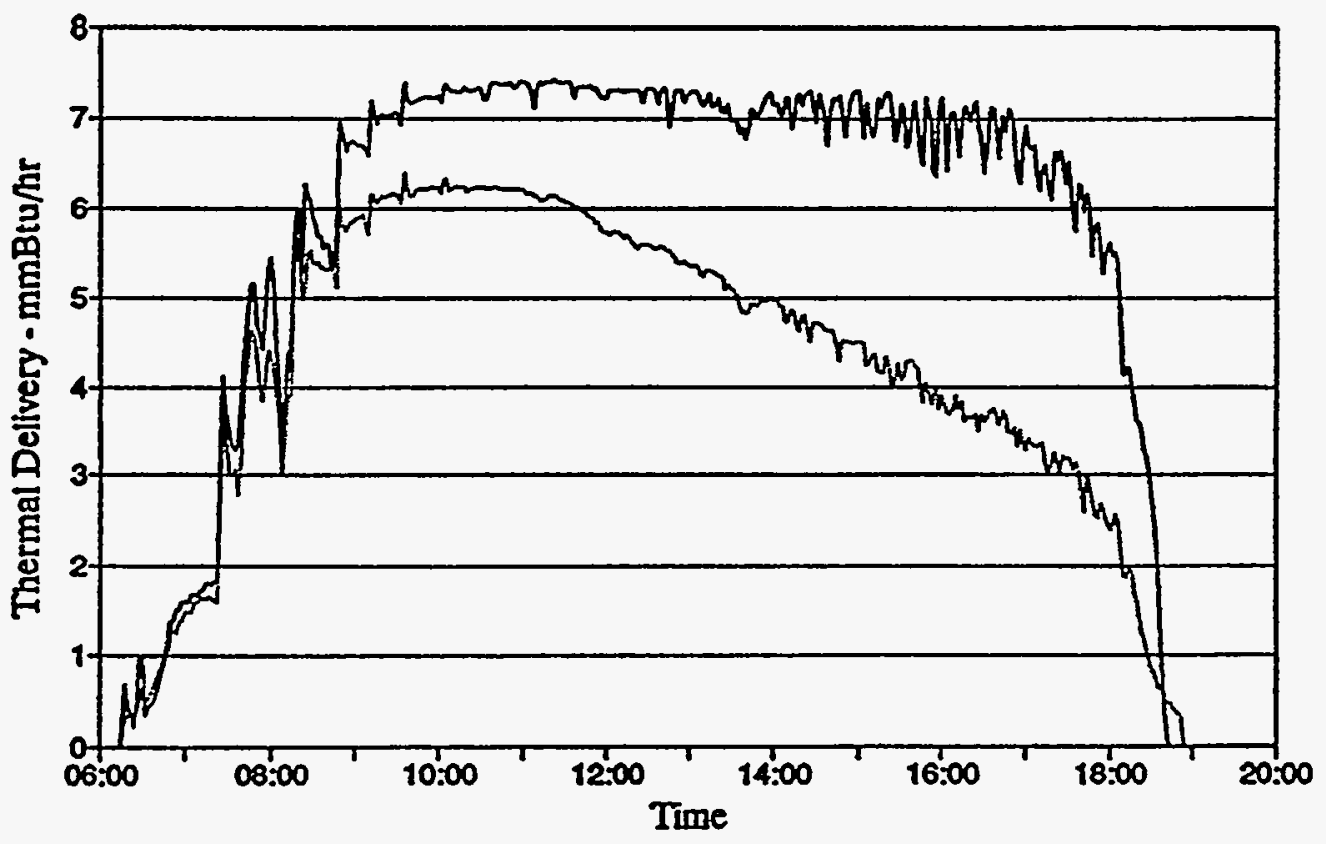

$$
-31+32 \text { A-D }-33+34 \text { A-D }
$$

Figure G-7. Addition of HCE alignment to test rows 33 and 34.

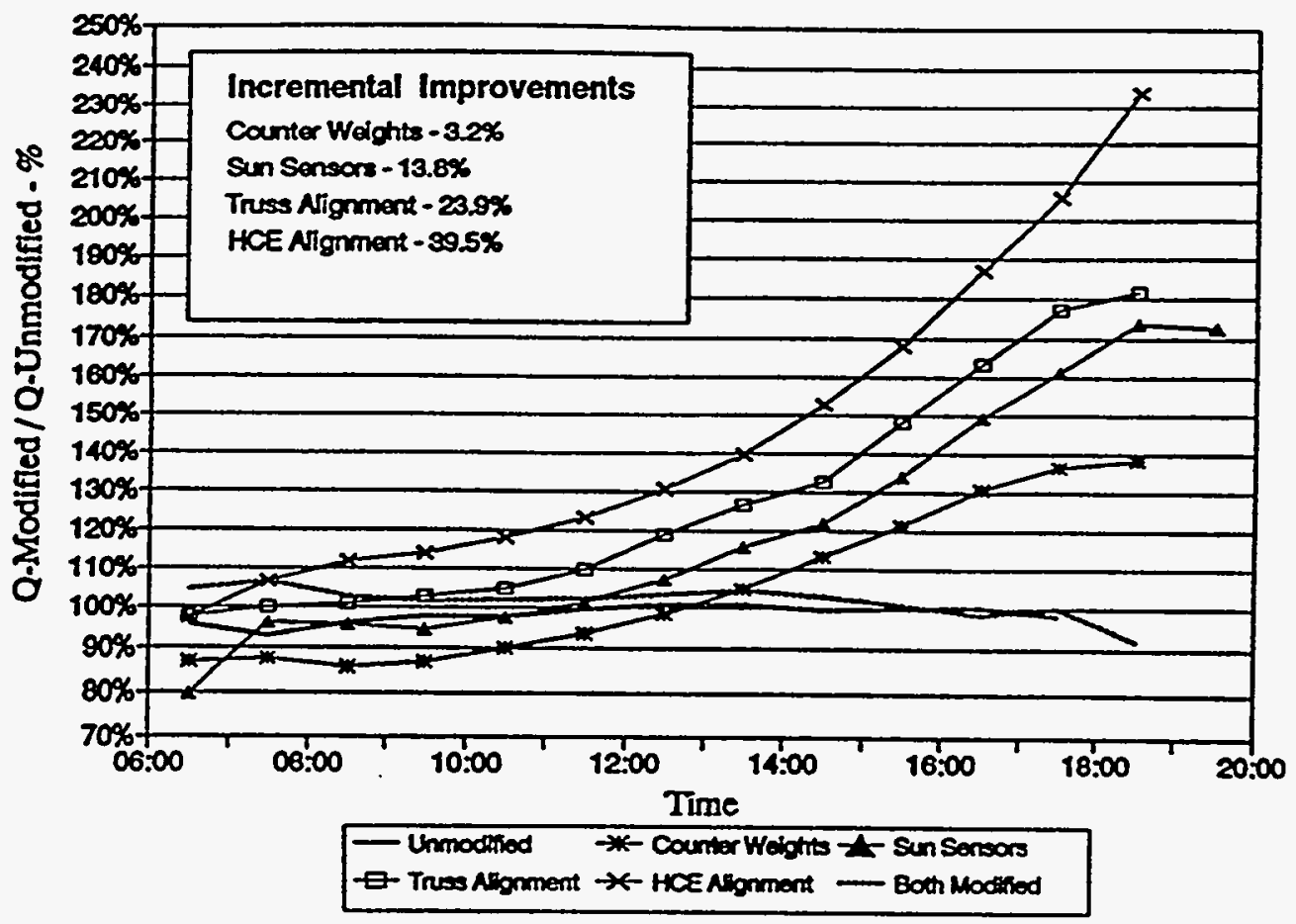

Figure G-8. Comparison of modified and unmodified test loops. 


\section{Phase 1 - Conclusions and Recommendations}

This series of tests has highlighted and quantified the importance of several maintenance actions that are possible with the LS-3 collectors in SEGS VII. With this information, decisions can be made on the cost/benefit trade-offs associated with specific maintenance activities. More generally, however, other conclusions can be drawn from this work:

- Several of the problems addressed by this effort were a result of faulty construction practice, which highlights the critical importance of demanding thorough and accurate quality assurance procedures during construction.

- Periodic checks and tune-ups appear to be warranted for the solar field collectors. A functioning test loop in a solar field permits periodic checks to determine the status of component alignments. With such information, an economic decision on maintenance actions to retune the solar collectors can be made. With current knowledge, it is our estimate that spot checks should be conducted annually, and realignment may be required every several years.

\section{Phase 2 - Test Description}

In the Phase 2 tests, we explored the tracking error that occurs on some SCAs because of a slight backward rotation of the SCAs after each forward tracking increment, apparently because of play in the gearbox. As a test, a grab brake was installed on the gear shaft of an SCA to stop this backward rotation, and testing was conducted to measure the effectiveness of this solution.

To conduct the testing, the two LS-3 test loops used in Phase 1 tests were used. One of the existing test loops, which exhibited good tracking characteristics, was chosen as the "base" loop. A second loop for the SEGS VII solar field, which exhibited poor afternoon tracking, was designated the "variable" loop; accurate flow and temperature instrumentation was moved to this loop to allow careful monitoring of the thermal delivery from the loop. Testing was then conducted to compare the performance of the two loops before and after modification of the variable loop with the brake mechanisms.

Improvement of the tracking accuracy will have a direct benefit on the thermal performance of the collector. An improvement of a few percentage points was anticipated.

\section{Phase 2 - Test Results}

Testing was conducted over a number of days in late summer to check repeatability of the results. Figure G-9 shows the comparison of thermal energy delivery between the base and variable loops for the original condition and after the modification of the variable loop with the brakes.

The use of the brakes improved the tracking. Before installation of the brakes, the tracking was uneven and erratic. After installation, tracking was much smoother and the thermal energy delivery improved. Quantitatively, the cumulative thermal delivery of the variable loop compared to the base loop increased 5.6\% during the hours from 10 a.m. to 4 p.m. 


\section{Phase 2 - Conclusions and Recommendations}

The brake mechanism is a viable solution to correct this particular tracking problem on the LS-3 collectors that exhibit variable tracking. Other solutions, such as a modification to the tracking software, may offer an alternative to correcting this problem.
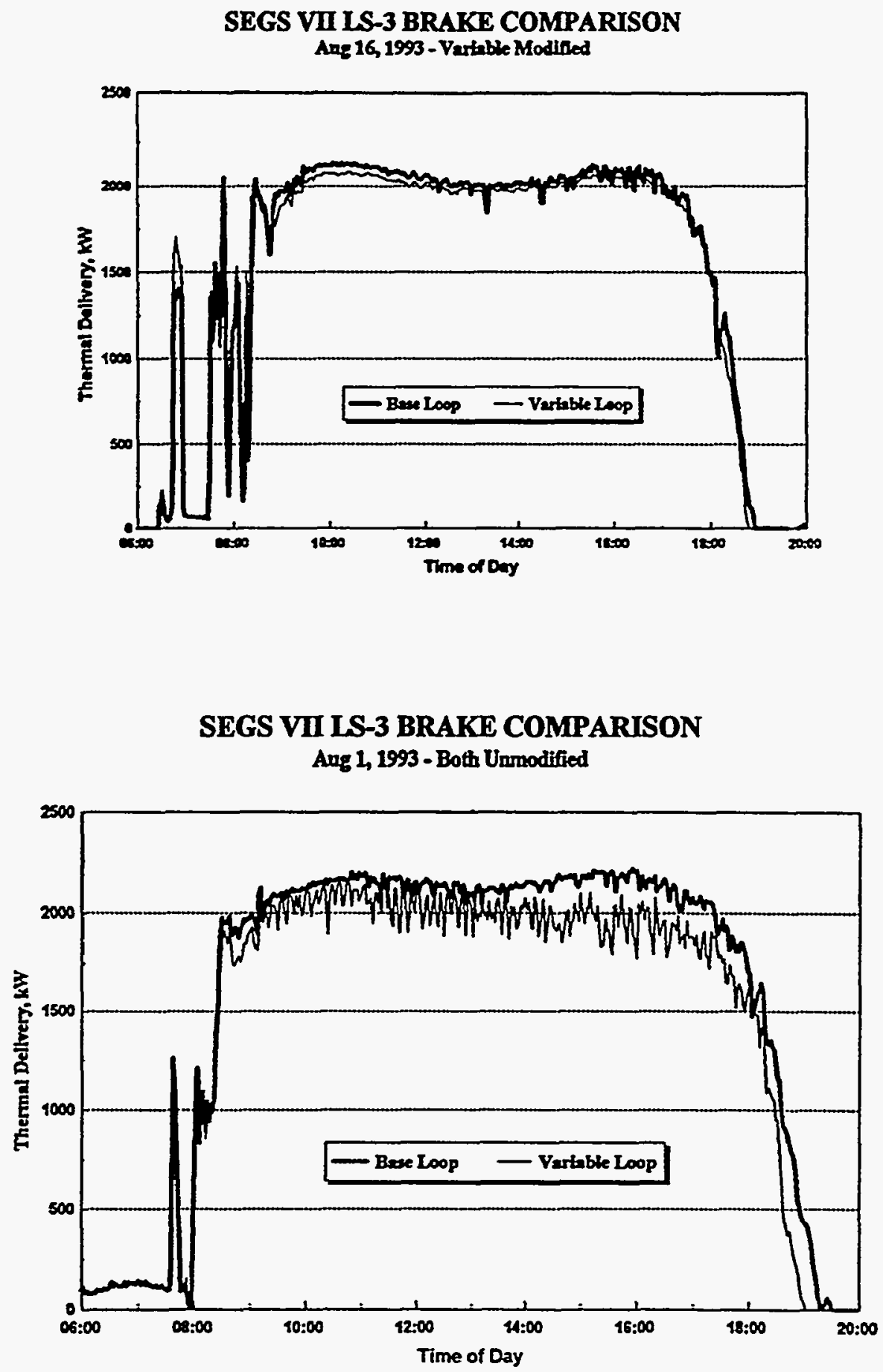

Figure G-9. Effect of brakes on the thermal performance of an LS-3 loop. 
This page intentionally left blank. 


\section{Appendix $\mathrm{H}$ \\ STRENGTHENED MIRRORS}

\section{Background}

Mirror panels at the solar electric generating system (SEGS) plants in California have exhibited excellent lifetime, without degradation of the silvered surface and excellent durability with proper design of the panels. One weak point, however, has been excess mirror breakage in high winds near edges of the solar fields, leading to excess operation and maintenance (O\&M) expenses. These expenses may be reduced with a modified design.

Mirror panels are attached to mirror support arms by pads adhered with an adhesive (the initial LUZ International Ltd. design used metallic pads and later designs used ceramic pads). Separation of the pads from the mirrors does occur, though it is not a serious issue with the ceramic design. The metallic pads have caused more problems because of the different coefficients of expansion between the glass and metal. Failures of weakened bonds, or even strong bonds, are aggravated under high-wind conditions when forces on the bond are high. The worst high-wind conditions are found at the edges of the solar fields, and mirror failures have a much higher frequency in these locations. It has been observed that glass breakage from high winds is seldom experienced on internal collectors where wind loads are reduced. It has also been observed that the impact of broken mirrors can "cascade" through the breakage of the heat collection element (HCE) glass envelope and/or other mirrors by falling pieces of broken mirror panel.

Two approaches were taken in the development of a strengthened mirror panel:

1. Build an entirely new mirror panel out of composite materials and a polymer reflector, or

2. Modify the design of the existing glass mirror reflector to improve its strength and prevent it from splintering if it breaks.

\section{Polymer/Composite Reflector}

Non-metallic mirror panels were ordered from Industrial Solar Technologies consisting of a silvered Teflon film from 3M Company (ECP-305+) and a composite layered aluminum and plastic structure. Between the cost-shared program and the O\&M budget, 1,500 panels were ordered. Final deliveries were made in the fall of 1995. While these panels showed excellent mechanical integrity during high winds, a bubbling of the silvered reflective film began to develop. The difficulty was traced to inadequate curing of the adhesive, and Industrial Solar Technologies undertook a program of refurbishment to correct the problem. Complete reinstallation of the refurbished panels was achieved in the spring of 1996. However, the durability of the ECP-305+ film is not acceptable and it has significantly degraded since reinstallation (see Figure $\mathrm{H}-1$ ). Use of this film is not recommended in future plants. 


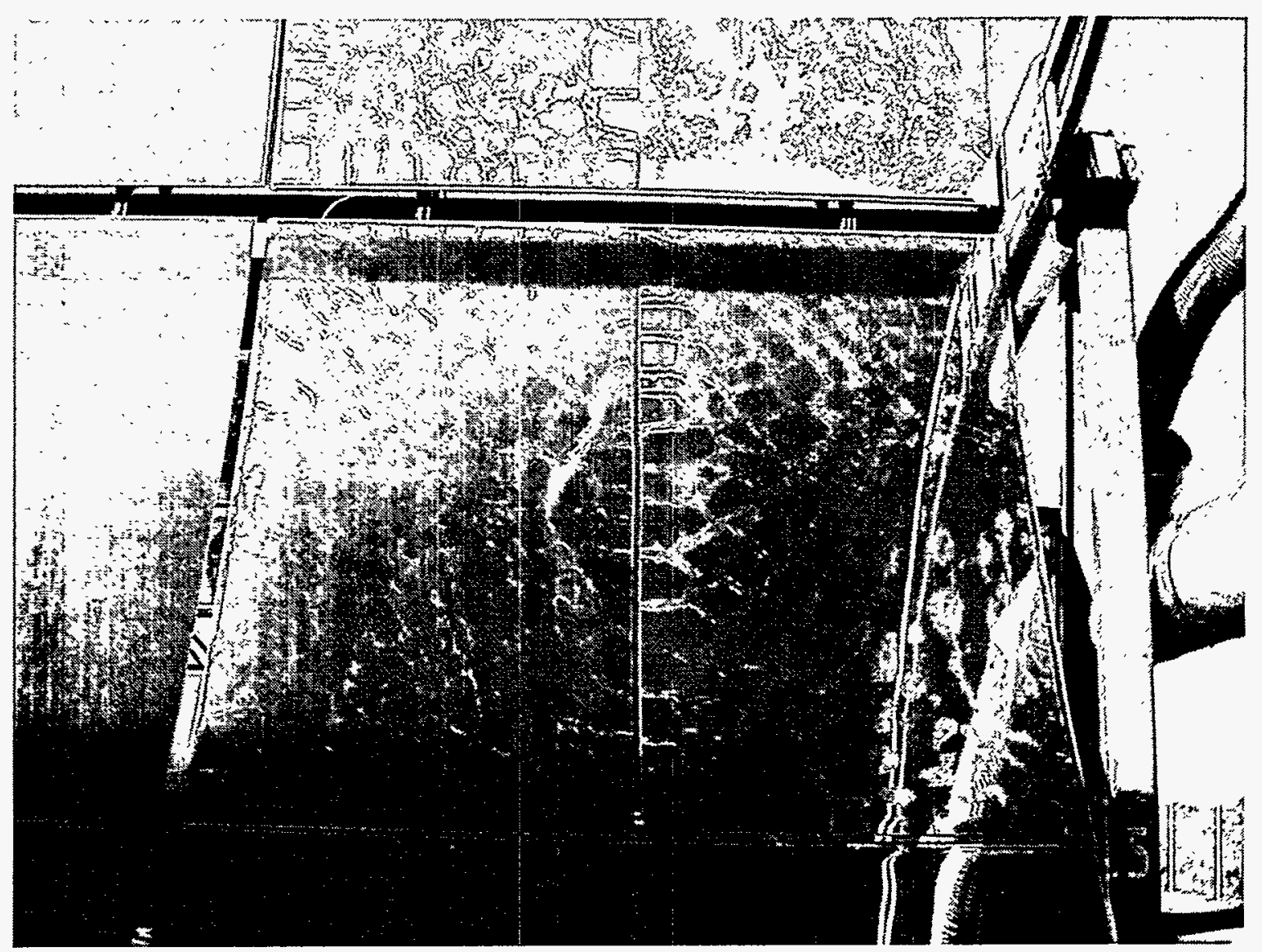

Figure H-1. Degradation of ECP-305+ reflector surface.

\section{Modifications to Existing Glass Mirror Panels}

In this task, we examined two methods of improving the strength of the existing glass mirrors. The design, effectiveness, and manufacturing cost were evaluated for each. The primary objective was to develop cost-effective designs with acceptably high benefit-to-cost ratios. The main steps in this task were the following:

1. Develop a concept for splinter-proof mirror panels.

2. Develop a concept for strengthened mirror panels.

3. Manufacture a prototype for splinter-proof and strengthened mirror panels.

4. Test the mirrors in the solar fields at the KJC Operating Company (KJCOC) site.

5. Test the materials at the Pilkington Solar International laboratories.

6. Evaluate the results from the site and laboratory tests.

\section{Description of Mirror Panels}

A total of 270 mirror panels is planned for on-site testing; the mirror panels are described in Table $\mathrm{H}-1$. The basic data for all mirror panels are listed in Table $\mathrm{H}-2$. 
Table H-1. Mirror Panel Description

\begin{tabular}{|c|l|l|}
\hline Number & \multicolumn{1}{|c|}{ Type } & \multicolumn{1}{c|}{ Enhancement } \\
\hline 90 & LS-3 inner segments & Splinter-proof \\
\hline 90 & LS-3 outer segments & Splinter-proof \\
\hline 25 & LS-2 inner segments & Splinter-proof \\
\hline 25 & LS-2 outer segments & Splinter-proof \\
\hline 10 & LS-2 inner segments & Fiberglass fabric/resin reinforcement \\
\hline 10 & LS-2 outer segments & Fiberglass fabric/resin reinforcement \\
\hline 10 & LS-2 inner segments & Fiberglass mat/fiberglass fabric/resin reinforcement \\
\hline 10 & LS-2 outer segments & Fiberglass mat/fiberglass fabric/resin reinforcement \\
\hline
\end{tabular}

Table H-2. Mirror Panel Data

\begin{tabular}{|l|l|l|}
\hline \multicolumn{1}{|c|}{ Component } & \multicolumn{1}{c|}{ Type } & \multicolumn{1}{c|}{ Specifications } \\
\hline Glass & Low-iron glass & $4 \mathrm{~mm}$ thick \\
\hline Reflective layer & Silver & $\mathrm{min} .0 .7 \mathrm{~g} / \mathrm{m}^{2}$ \\
\hline Protective layer & Copper & $\mathrm{min} .0 .3 \mathrm{~g} / \mathrm{m}^{2}$ \\
& Base lacquer & $40 \pm 10 \mu \mathrm{m}$ \\
& Intermediate lacquer & $40 \pm 10 \mu \mathrm{m}$ \\
& Finish lacquer & $40 \pm 10 \mu \mathrm{m}$ \\
\hline Reflectivity & Moon air mass 2 & $>92 \%$ \\
\hline Mounting element & Material & Ceramic \\
& Adhesive & Silicon \\
& Tensile strength & $>0.62 \mathrm{~N} / \mathrm{m}^{2}$ or $1.95 \mathrm{~N} / \mathrm{pad}$ \\
\hline
\end{tabular}

\section{Splinter-proof Mirror Panels}

A material for the splinter-proof layer was selected. Mirrors with this layer were produced and shipped to KJCOC in February 1997. The layer consists of adhesive foam strips covered with aluminum. The selected highly durable double-sided adhesive polyethylene foam is able to pass the automotive requirements for interior and exterior use. The pure acrylate adhesive has excellent adhesion and a high final strength as well as good temperature, aging, and plasticizer resistance. The combination of the adhesive with the compressible, permanent elastic foam ' results in a high application safety based on a large effective adhesion surface. The adhesive foam dampens vibration and has an excellent resistance to ultraviolet light as well as a good weathering resistance. Because of the aluminum layer on the topside, the material has additional ultraviolet protection.

The foam strips are applied as shown in Figure $\mathrm{H}-2$. 


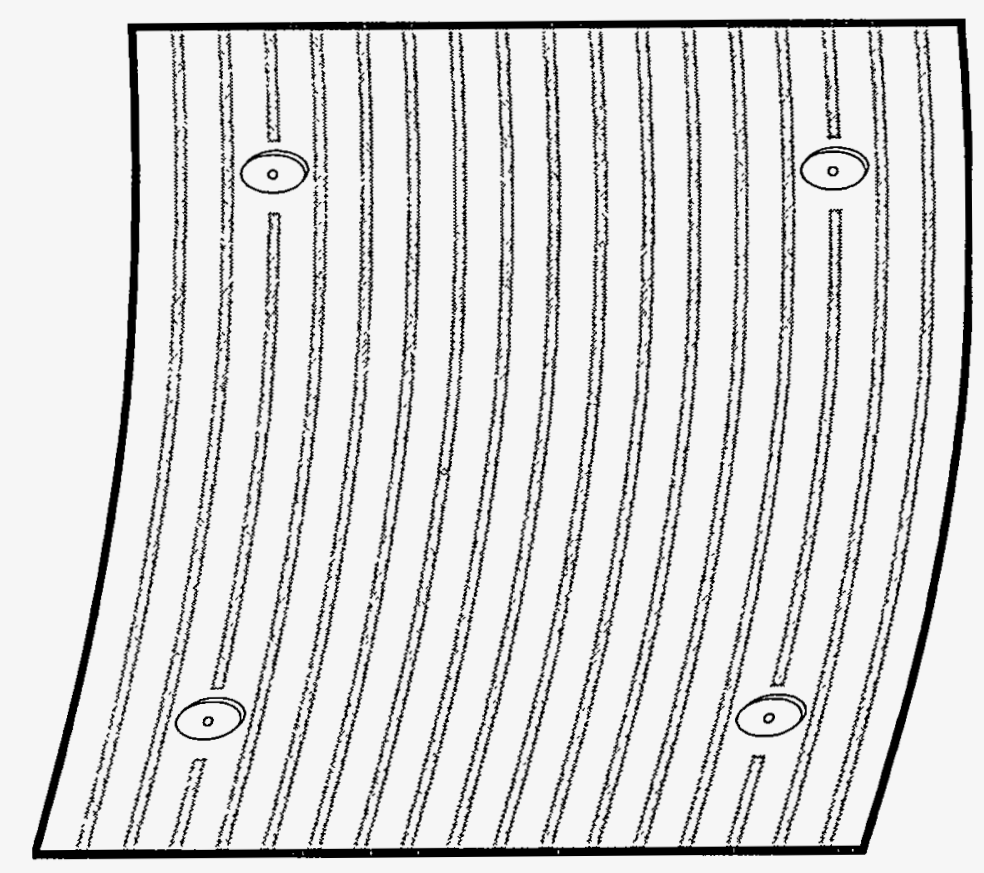

Figure H-2. Splinter-proof panes with foam strip reinforcement.

\section{Strengthened Mirror Panels}

The materials for the reinforcement layers were selected, and mirrors with this layer were produced and shipped to KJCOC in March 1997. The reinforcement is achieved by a fiberglasstype layer with an ultraviolet-proof coating. The reinforcement laminate is then applied instead of the finish lacquer. Two different types of laminate are being tested. The "one-layer reinforcement" is designed to provide both splinter-proof and strengthening properties (see Figure $\mathrm{H}-3$ ). The second fiberglass layer in the "two-layer reinforcement" should further reduce the risk of breakage.

The one-layer reinforcement consists of

- A $270-\mathrm{g} / \mathrm{m}^{2}$ fiberglass fabric/resin layer

- A $10-\mathrm{cm}$ wide strip of $300-\mathrm{g} / \mathrm{m}^{2}$ fiberglass mat/resin layer along the edges

- An ultraviolet resistant topcoat layer

The two-layer reinforcement consists of

- A $270-\mathrm{g} / \mathrm{m}^{2}$ fiberglass fabric/resin layer

- A $450-\mathrm{g} / \mathrm{m}^{2}$ fiberglass mat/resin layer

- An ultraviolet resistant topcoat layer 


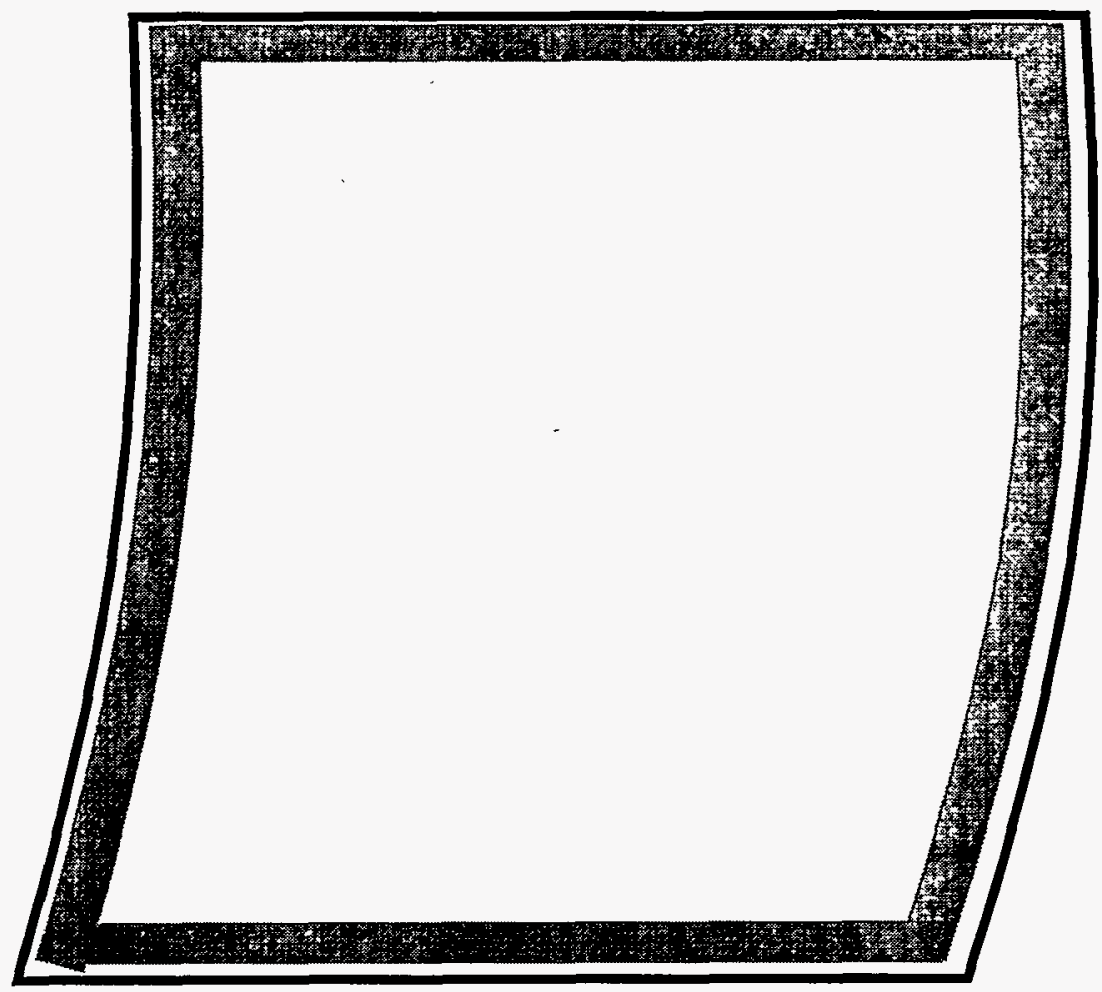

Figure H-3. Strengthened panels with one-layer reinforcement. 
This page intentionally left blank. 


\section{Appendix I \\ POWER PARK DATA NETWORK}

\section{Background}

Because the complexity of solar plant operation has increased, rapid information access, information processing, and data transfer are essential to maximize operating objectives. Historically, these functions were hindered because data collection was done manually, requiring a large work force and resulting in significant delays in information transfer. While the solar electric generating system (SEGS) projects used various computerized and manual systems to monitor and control the various functions of plant operation, many of these systems provided limited or no capability for recording, transfer, or analysis of plant data, making it impossible to implement a consistent performance monitoring program. The lack of an integrated data collection system also resulted in limited access to historical plant operating data. Consequently, operators or plant performance engineers could not, for example, easily compare current data to similar data of the previous year.

Access to a centralized database allows more rapid identification of potential problems, more informed operating strategy decisions because of improved data availability, and the ability to quickly alter plant operating plans because of changes in weather or equipment problems.

All of these reasons pointed to the need to develop automated systems to provide operation and maintenance personnel with the best possible information in a timely manner to enable optimum plant performance at a minimum cost.

While these observations and arguments pointed to the value of a network for the Kramer Junction, California, SEGS plants, they become even more powerful for a solar power park consisting of a number of solar power plants with integrated control functions in a single or limited number of control rooms. Thus, the concepts and methods developed in this task should have important applicability to a power park configuration in which operational and control system information transfer are not only more critical, but whose volume and scope are also more complex.

\section{Scope}

This task provided a site-wide PC-based computer network that integrated the various process and plant interface computers. The following applications, consisting of both Sandia National Laboratories and $\mathrm{KJC}$ Operating Company (KJCOC) program activities and normal KJCOCfunded activities, were developed to operate over the network to improve electronic plant information management:

- Operator log database (see Appendix O)

- A computerized maintenance management system (see Appendix K)

- Solar field maintenance management (see Appendix L)

- Accounting and Payroll (KJCOC-only task) 
- Company forms (KJCOC-only task)

- Employee, safety, environmental, and other plant databases (KJCOC-only task)

- Electronic mail (KJCOC-only task)

\section{Specific Objectives}

Our specific objects are as follows:

1. Install a PC network that connects each of the control rooms with the main administration and maintenance office complexes.

2. Install a file server on the network to allow centralized storage of data and software.

3. Make the network expandable to allow all PC users to be connected.

4. Provide operator workstations in each control room.

5. Make the network capable of interconnecting with the various plant process computer systems to allow automatic downloading of process data over the network.

\section{Network Design}

\section{Site Layout}

The Kramer Junction site is unique for a power plant in that large distances separate the control buildings, maintenance shops, and administrative complex. A single run of 4,600 feet is required to connect two of the control buildings, with a total run of 13,400 feet to connect all control buildings with the administrative complex.

\section{Network Hardware}

Ethernet protocol hardware was selected for the network because of its low cost, flexibility, expandability, and high communication speed. An Ethernet network card is installed in each computer connected to the network. Printers connected to computers tied into the network can be set up to be used by any users on the network, provided the printer host-computer is turned on. Some printers can also be connected directly to the Ethernet network by inserting a special network printer card. Both types of printer access will be used in this network.

Because of the large distances that the network must span, a fiber-optic highway is used to connect each of the control buildings and administrative complex. A direct burial fiber-optic cable was used to make all runs. The fiber-optic highway connects to Ethernet hubs in each of the control buildings and the administration building. These hubs allow up to 24 devices (computers and printers) to be connected to the network in each control room and 48 in the administration building. Additional devices can be added to the network by adding additional standard Ethernet hubs. Computers and printers are connected to the hubs using standard $10 \mathrm{base} T$ (telephone) connections. The fiber-optic highway is set up in a loop to allow redundancy in case any of the fibers are damaged. Special monitoring software is used to 
monitor the fiber-optic highway and hubs should any problems arise. Figure I-1 schematically shows the network plan.

\section{Network Software}

Novell software was selected for the network operating system. Most software and hardware can be made to operate with Novell. Novell has built-in security to allow the network administrator to give individual users different levels of access.

\section{File Server}

The network file server runs the Novell operating system. It is also used to store data and software for all users to access. The file server computer has an ESIA bus (32 bit) and three ESIA network cards to ensure rapid data transfer. The large amount of RAM is used as a disk buffer to minimize disk reads and writes to increase data throughput.

\section{User Workstations}

All existing PC computers can be connected to the network. Users can run either DOS or Windows.

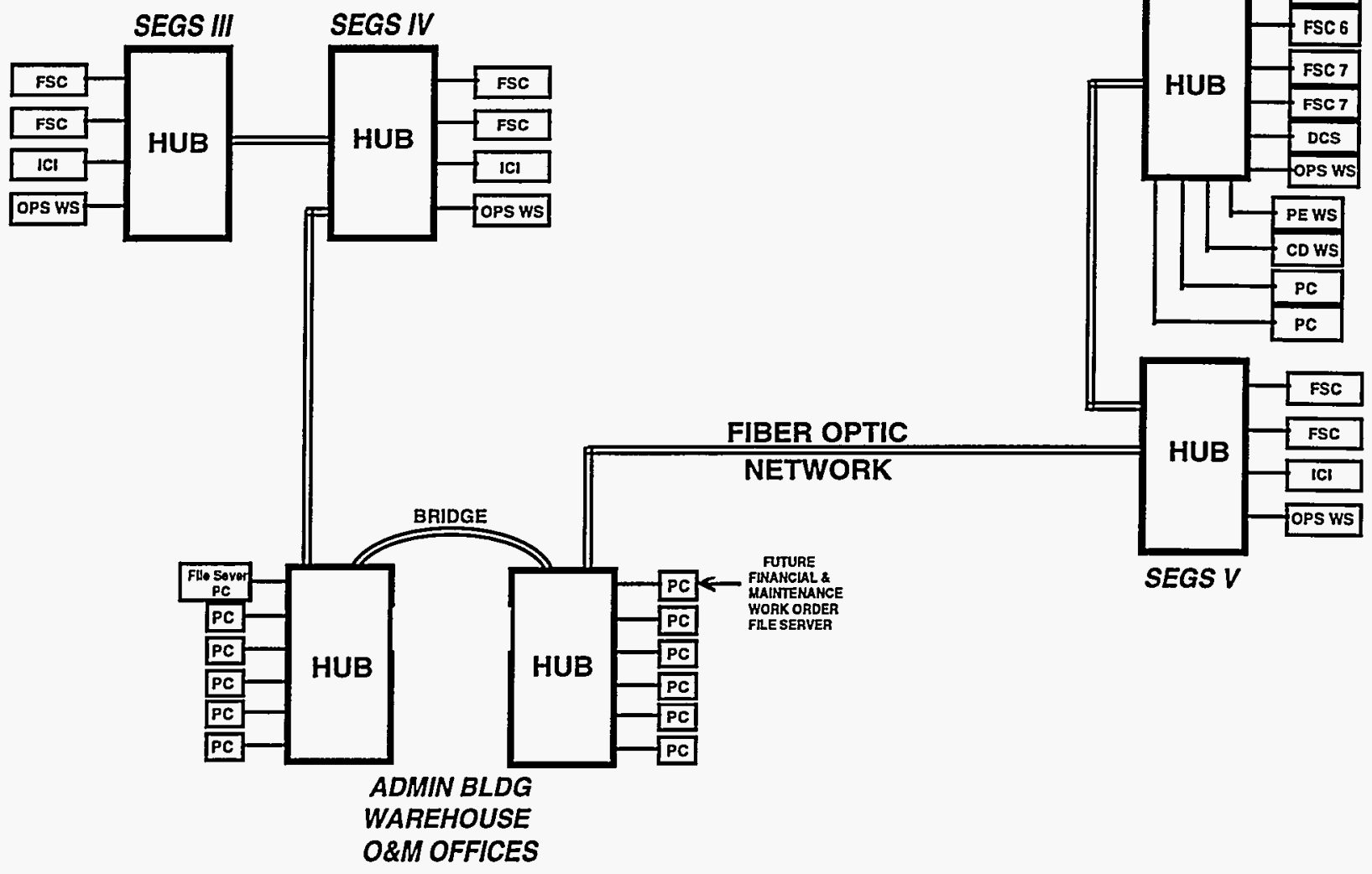

Figure 1-1. The Kramer Junction data network. 


\section{Benefits}

\section{General}

The PC network provides the backbone for the Plant Information Management System. The network allows for improved information transfer, improved on-line analysis (when other subtasks are completed), and automatic data transfer. Network installation will increase plant performance and reduce the work force required for data collection and data entry.

In addition to the above reasons, the network will pay for itself by reducing software costs, hardware costs, and improving data recovery. There were some increased costs in training individuals to work on a network; however, these were offset by improved productivity resulting from standardized interfaces. When running software over a network, the software is physically located on the network server and is loaded over the network to the individual's workstation when the program is accessed. The software is generally run on the individual's computer, but some software can be run on the server as well.

\section{Reduced Software/Hardware Costs}

The number of copies of software that must be purchased can be reduced because a site only requires as many licenses as the maximum number of people using that software at any time rather than a separate copy for each user.

Network versions of software are cheaper per user than are individual packages. Furthermore, upgrades to software are cheaper when purchased in volume and can be accomplished more quickly on a server. The network allows certain hardware to be shared, such as printers, modems, and faxes.

Windows and other new software require a large amount of hard-disk space. Workstations on the network load the software off a file server; the workstations do not require as large a hard disk.

\section{Improved Office Efficiency}

The network allows data files or documents to be shared so that individuals or groups can work on the same document. In addition, each user can have daily backup of data if it is stored on the server. An individual can use any PC on the network to perform work if his/her own computer fails to function properly.

The ability to send and retrieve data to and from the file server provides another important efficiency. An example of this is the distribution of reports. Reports are generated and then placed onto the file server for all authorized users to view at their leisure. If an individual wants a hard copy of the report, it can be printed on the nearest printer. This saves work-hours in printing, photocopying, and distributing reports.

\section{Standardization of Software}

The file server is loaded with the appropriate software that the company wishes to use, which will allow all employees to have access to the same software. The program software is loaded 
only on the file server and not on every PC; therefore, fewer work-hours are required to load and upgrade software.

\section{Power Park}

A network will be an essential element of a solar power park. The factors cited here-physical distance, volume of information, need for rapid access to data, data acquisition, data logging, and data analysis-will be magnified in a power park setting.

\section{Disadvantages}

To reap the full advantage of the network, KJCOC had to upgrade its "outdated" computers; however, current PC systems will still be able to connect to the network. As old systems fail, they are replaced with the more advanced models.

Training on effective network use was required for most users because of the added complexity of a network system versus a stand-alone PC system. This complexity also leads to the need for a part-time system administrator on-staff.

Fiber-optic cabling is fragile and expensive to repair. If too many repairs are made, the signal through the cable will be too weak and therefore must be replaced. Additional equipment making up the network system must also be maintained.

\section{Conclusions and Recommendations}

The network at Kramer Junction was successfully implemented and is continually being improved. Network design was assisted by the early involvement of consultants and other independent reviews.

Networks, although they are not particularly complicated, are not completely understood by the average microcomputer user. There are a significant number of network hardware and software options available on the market today, and there are no obvious right or wrong choices when designing and setting up a network. Thus, it is essential that someone with significant knowledge of networks be involved in the design of a new network installation. This will help to ensure that the network is designed to meet the requirements of a particular installation. In our view, it is better to have access to an independent consultant in contrast to relying on vendors or a self-educated employee.

While it is straightforward to discuss the qualitative benefits of a network, it is difficult to quantify the payback resulting from the installation of the network. The network itself is more of a part of the plant infrastructure than a process component. Its value will depend more on how it is used and the quality of the applications that are used on it. However, the intangible benefits, such as improved communication, data sharing, and access to plant information, are significant. Savings that are more tangible can be calculated from reduced hardware and software costs. The productivity of individuals will decline at first during the familiarization phase and then improve as skills improve and network capabilities are implemented. 
The following observations seem appropriate:

- A network should be considered an integral and necessary part of a solar power plant and should be part of the initial plant design.

- The Ethernet architecture appears to be a good choice from cost, performance, and interconnectivity standpoints.

- Fiber-optic highways are a good choice for long runs.

- Plant process-control computer systems should be designed to link and communicate with the network.

- During project engineering, efforts should include the development of plant information systems that can be accessed over the network, such as:

- A computerized maintenance management system;

- Financial tracking packages;

- Documentation (drawings and specifications);

- Operating procedures;

- Other information databases.

- Radio modem or wireless local area networks (LANs) for mobile access to the network should be investigated.

- The file server is the heart of the network. The following characteristics help optimize the performance of the file server:

- Buy a machine designed to be a server.

- Maximize the file server RAM.

- Use multiple 32-bit network cards in the file server.

- Assuming that there is never too much disk space, install the capability to expand to 4 or 5 GB of hard disk capacity.

- Install a system for daily backup or use disk mirroring to protect data on missioncritical applications. 


\section{Appendix $\mathbf{J}$ \\ INTERTIE OF PLANT CONTROLS TO POWER PARK DATA NETWORK}

\section{Background}

After the power park data network was installed (see Appendix I), some plant control systems required upgrading to allow interconnection with the network. The Operation and Maintenance Improvement Program (O\&MIP) funded the upgrade of control systems in two phases, as described in the following sections.

\section{Phase 1 - Upgrade of Instrument Controls Interface}

The instrument controls interface (ICI) systems at Solar Electric Generating Systems (SEGS) III through V were obsolete systems for monitoring and control of the heat transfer fluid (HTF) system and various power block subsystems. Plant operators had access to only limited data and had no ability to examine trends, pull up historical information, or scrutinize major components for performance characteristics. The old control system did not allow intertie with the new site network described in Appendix I. Plant performance suffered because of this lack of information and control.

The objective of this task is to upgrade the ICI system at SEGS III through V to significantly improve operator data and control capabilities and to allow interface with the site data network.

\section{Phase 1 - Scope and Objectives}

This task replaced the old ICI with a modern distributed control system (DCS) to upgrade operator control and information. In selecting the new system, desirable secondary objectives were to standardize the spare parts, training, and service with the SEGS VI through VII DCS.

Specific objectives included the following:

1. Develop specifications for the new DCS at SEGS III through V.

2. Competitively select a vendor for the new DCS.

3. Procure, install, and implement the system at SEGS IV on the Sandia National Laboratories/KJC Operating Company (KJCOC) cost-shared program, and on SEGS III and $\mathrm{V}$ with owner funds.

\section{Phase 1 - Results}

Equipment designed and manufactured by the Westinghouse Process Control Division (PCD) was chosen for this project, partially for conformity with the existing DCS at SEGS VI through VII. Each new DCS was a Westinghouse PCD 002 WDPF system, consisting of a distributed processing unit (DPU), a data highway, a data highway controller, an operator workstation, an engineer's work station, and a historian station. The DPU has a 32-bit functional processor with floating point and gate array co-processors to perform data acquisition, modulating, and 
sequential control functions. The operator workstation displays custom and standard graphics and controls; the engineer's station allows changes in configuration and maintenance of the system; and the historian station collects and stores process data. Each plant has an operator's station and the plants share one engineer's station and one historian. A schematic diagram of the system and a typical control room layout are shown in Figure $\mathrm{J}-1$.

The Westinghouse PCD equipment was accepted in December 1993 by KJCOC, and operation on the new DCS was fully implemented at SEGS III in 1994.

The number of control/monitoring points was increased from 137 to 430 . Data screens now available to the operators include the HTF system, the steam/water system, the raw water system, the HTF pumps, the cooling water system, subscreens for various control valves, electricity and gas meter readings, and solar field thermal calculations.

\section{Phase 2 - Upgrade of Field Supervisory Controller}

The field supervisory controller (FSC) is the hardware and software that supervises the operation of the solar field. Each SEGS plant has a solar field comprised of approximately 1,000 solar collector assemblies (SCAs); each SCA is an independent solar collector with its own microprocessor or localized controller (LOC). The LOC controls the normal tracking operation of the SCA. Each LOC in turn communicates with the FSC in the power plant control room. The FSC supervises the operation of the LOCs; in fact, the LOCs are slaves to the FSC and will stow a collector if communication is lost to the FSC.

During normal operation, the FSC tracks the theoretical position of the sun. When the sun gets to 10 or 11 degrees above the eastern horizon, the FSC sends a deploy (or wake up) command to each SCA in the solar field. At this point, the LOC takes over the operation of the SCA and will continue to track the sun for the entire day or until it receives another command from the FSC. The LOC communicates with the FSC throughout the day, updating the FSC on its status (temperature, mode of operation, or alarms). Some safety protections are built into the LOC, such as overheat protection or loss of communication with the FSC. Other safety protections built into the FSC are checks for high wind speed conditions, low or loss of HTF flow, and mirror burn protection.

\section{Phase 2 - Scope and Objectives}

A task was completed in 1994 with the following objectives:

1. Upgrade and standardize the FSC computer hardware and software."

2. Eliminate existing program bugs and improve the operator interface.

3. Improve reporting and information reduction.

4. Integrate with site network for data transfer.

5. Allow for remote monitoring of multiple plants from a single network station, e.g., another control room or by the plant manager.

\footnotetext{
* The new FSC software is protected by a KJCOC copyright.
} 


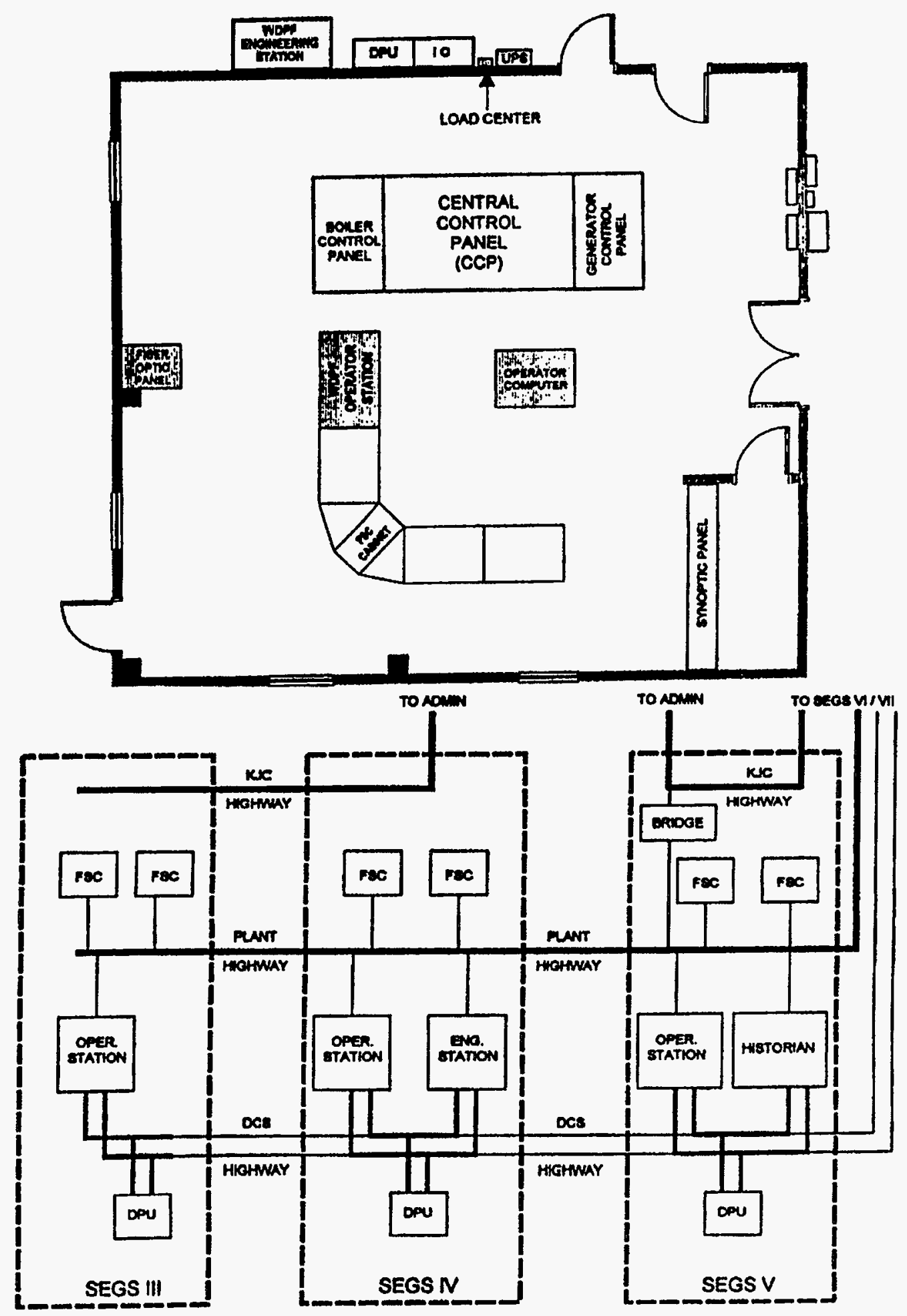

Figure J-1. System schematic and typical control room layout. 
The new FSC is an IBM PC-compatible computer written in Microsoft Visual Basic. Some of its many new features include automatic switching to a backup computer with loss of capability; improved operator interface; and improved data collection, analysis, and reporting capabilities.

The purpose of this task was to further expand the capabilities of the FSC by

- Linking this unit directly with the DCS and the solar field maintenance program, and

- Developing the ability to view the FSC for any plant on-line via the plant data network, allowing managers, engineers, and supervisors the ability to view solar field conditions from outside the control room.

\section{Phase 2 - Results}

The FSC was successfully linked with the DCS in mid-1996. Remote viewing of the solar field was also satisfactorily completed with the development of the "SEGS-VIEW" program. This program allows authorized users to monitor and analyze on-line status of the solar fields, but does not allow remote control. The main screen of the program is displayed in Figure J-2.

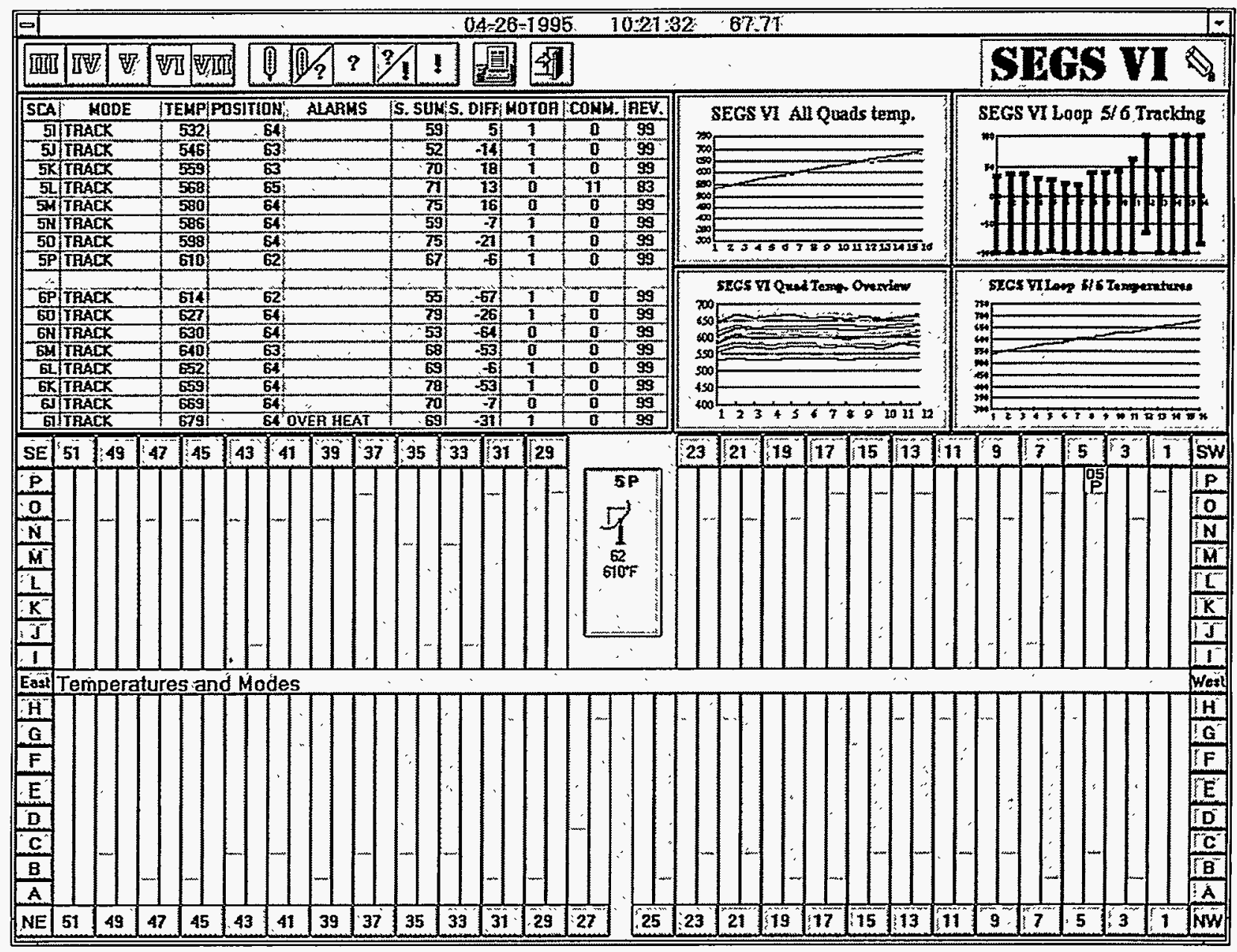

Figure J-2. Main screen of the SEGS-VIEW program. 
The backup FSC at each plant sends the current field information to the network every 20 seconds, which is about the time it takes each primary FSC to refresh the display of the entire field. Therefore, the data on the network is virtually immediate. The program lets the user select the plant to monitor, using the toolbar at the top of the screen. It also allows selection of the type of information to display graphically. Five options are available, similar to the actual FSC display. The user can select the following:

- Temperature/Mode

- Temperatures only

- Modes only

- Modes and alarms

- Alarms only (this is actually a display of the nontracking SCAs with their current mode) 
This page intentionally left blank. 


\section{Appendix K COMPUTER MAINTENANCE MANAGEMENT SyStem}

Successful implementation of Computer Maintenance Management System (CMMS) methodology required that the plants and administrative functions be integrated on a comprehensive computer network. Consequently, completion of the data network (see Appendix I) was a necessary prerequisite to completion of this task.

Maintenance planning has taken various forms at the Kramer Junction, California, plants over the years of their operation, depending on current plant management. Though this is a generalization, the previous process can best be characterized as planning without effective integration of the important functions, e.g., maintenance, operations, purchasing, and stores. Such integration is necessary for reasonable planning, and the CMMS approach ensures-even forces-a close coordination between functions. Past difficulties include specific incidents where parts were not available, information from previous repairs was not readily available, or the maintenance and operations groups were at odds over what needed to be done, and when. With a functioning CMMS, such issues are almost nonexistent. In addition, the approach at Kramer Junction is that Operations sets the priorities for maintenance activities, and Maintenance carries out the work. Evaluation of this process led to the recognition that an integrated process that starts with work order preparation, e.g., from the Operations staff, and proceeds through execution via a controlled and traceable system could have a significant influence on savings of operation and maintenance (O\&M) cost and reduction of work-force needs.

To achieve this goal, a systematic evaluation was made of existing CMMS. A commercial product called Maintenance Planning and Control (MPAC) ${ }^{1}$ was chosen from four finalists. Separate from the O\&M Improvement Program, MPAC was also integrated with a new accounting package called COGENT. The total system consists of four highly integrated modules for maintenance, warehousing, purchasing, and accounting. These modules incorporate functions such as a master equipment list, work-order system (both corrective and preventive), purchase orders, stock issue requests, work-force planning and scheduling, inventory accounting, warehouse management, and all tracking for accounting purposes. Users can access important data from any point in the system. A simplified illustration of the system interactions is given in Figure $\mathrm{K}-1$. MPAC is also a powerful tool for tracking projects with multiple tasks (such as the O\&M Improvement Program).

\section{Selection of MPAC as the CMMS Package}

To select a CMMS, maintenance literature was searched and several power plants were visited to learn firsthand about available tools and packages. In addition, two consulting firms were engaged to help in the process. One firm reviewed the requirements that KJC Operating Company (KJCOC) should consider for new software for both accounting/financial and maintenance management. The second firm provided some additional information ${ }^{2}$ on comparative

\footnotetext{
1 "MPAC Overview," The System Works, Atlanta, Georgia, August 1992.

${ }^{2}$ For the comparative table used, see Maintenance Technology, February 1991. See also D. B. LeMaster, Improved Maintenance Management Practices, APPA Engineering \& Operations Workshop, Orlando, Florida, February 27, 1991.
} 


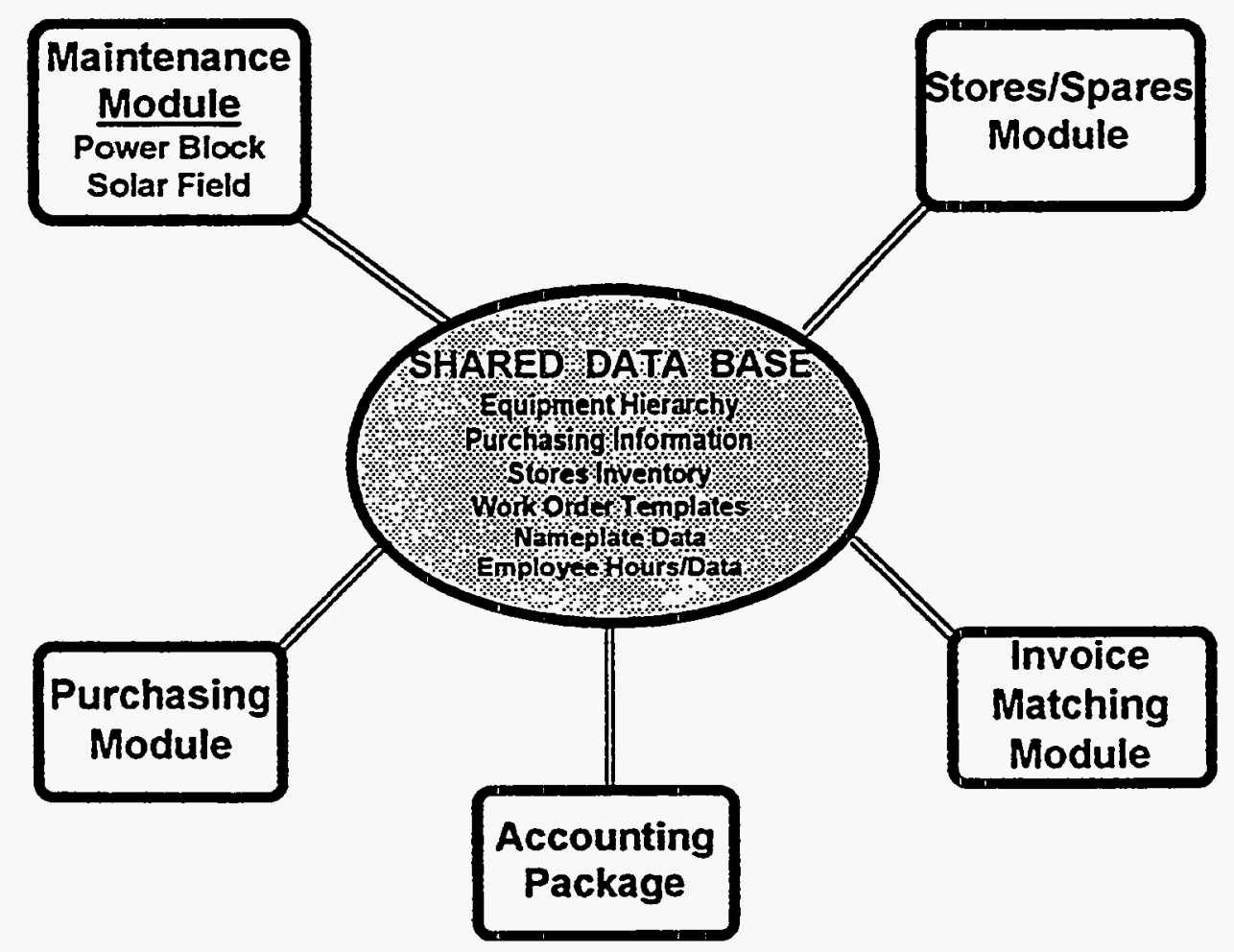

Figure $\mathrm{K}-1$. O\&M maintenance planning functions and interrelationships.

data on CMMS software. A yes/no-type comparison looked at characteristics on functions, software features, database source, hardware, operating system, and training and support. Using this input, $\mathrm{KJCOC}$ selected four systems for vendor presentations to key staff at the site: Maximo Series 3, MPAC, EASE, and Mapcon. All are PC-based, reasonably flexible, provided by a vendor with at least a ten-year track record, and use an ASCII format to export data to an accounting package. Costs of such systems vary considerably depending on complexity and configuration, approximately ranging from $\$ 50,000$ to $\$ 300,000$. After the presentations, MPAC was clearly favored by the staff because of the depth, flexibility, and power of its features.

\section{Results}

The MPAC system is complex and it took approximately two years to fully implement the system at the Kramer Junction site.

It is difficult to assess the full impact of the new methods at this time. While procurement, maintenance, and tracking procedures are much improved and the annual O\&M budget reduced, there are factors other than the O\&M Improvement Program alone (e.g., owner's policies, internal management policies, and functioning) that have strongly influenced the current status of O\&M planning and cost. However, a few remarks regarding progress are in order.

Maintenance procedures and implementation - from recognition of a problem to final resolution-are recognized at the plants to be more efficient in terms of time, effectiveness and, inherently, cost. The procedures and data flow are considerably improved from planning to parts or services procurement to maintenance implementation. The procurement process, which includes purchasing, stores and inventory, and accounts payable, now requires approximately $30 \%$ less staff. Purchasing procedures are faster and approval requirements tighter than before, 
though the computerized system allows the process to move smoothly and quickly (from request to purchase order) in one-half day or less, if required. Parts tracking and inventory planning are considerably better: the maintenance staff can check availability of parts; fill rate (availability on site) is improved; control of physical inventory and the tracking and identification of highvolume or critical parts are more effective and comprehensive; and the procedure by which maintenance staff gains access to parts in stores is much better. The process from request of a part or service to final payment is seamless and much less subject to error. With these systems in place, the maintenance planners have much more information at hand and are able to plan maintenance activities more efficiently.

It must be emphasized that it is as much the people as the methods that have brought about these improvements; but without implementation of the new system, progress would have been much more difficult and it would have been impossible to attain the present level of O\&M planning and control.

While the MPAC tool has greatly improved the efficiency of O\&M management, the older version software implemented by KJCOC was not as user-friendly (i.e., not Windows-based) as current versions of MPAC or other competing CMMS software, and was too costly to purchase and maintain relative to a CMMS that $\mathrm{KJCOC}$ would recommend today. Consequently, $\mathrm{KJCOC}$ may replace MPAC with an alternate CMMS product in the near future. 
This page intentionally left blank. 


\section{Appendix L SOLAR FieLd MAINTENANCE PLANNING}

KJC Operating Company (KJCOC) has continued to improve its solar field maintenance program to maximize solar field availability and minimize work force and material costs. This improvement is demonstrated by

- Increased recognition of the cost-effectiveness of gathering high-quality and consistent historical data.

- Emphasis on experienced and dedicated maintenance crews.

- Exploration of new remote data transmission techniques and computer software for gathering solar field data (see Appendix M).

Solar field maintenance planning uses a group of field operators from the Operations Department that visually inspects the solar field components on a continuous cycle on day shifts. Any collector malfunction or discrepancy is noted and put into the monitoring system and computerized database. The database includes codes for fluid system components, electrical and electronic components, grounds, and mechanical/structural/reflector components. Codes have been established to describe, at the subcomponent level, the overall status, repair status, and priority for solar field component maintenance items.

All solar field maintenance activities use this database to set work priorities. Generally, this system ensures that a solar collector assembly (SCA) would get checked about three times a month, while critical components such as flexible hoses, heat collection elements (HCEs), and mirrors are subject to additional targeted inspection. The solar field maintenance planning tool is used by Technical Services and Operations to identify and prioritize solar field maintenance requirements for Planning, and to analyze the failure modes and failure frequency of solar field components.

Within this task, the system of codes for equipment status, repair, priorities, and component identification numbers was redefined and a new software system - the Field Status Program (FSP) - was developed and implemented.

The FSP allows the solar field data to be easily entered into a system by component, status, problem, priority, and corrective action taken. The software is image-oriented such that the solar field operators select a component from a visual image and option list, rather than enter the codes directly. Consequently, solar field operators inspect the field with a mobile computer described in Appendix $\mathrm{M}$ and enter data that are subsequently loaded into the plant data network. Once entered, these data are immediately available for analysis and for use in the maintenance planning function. Defects noted as immediate priority are dealt with immediately by Maintenance.

Figures L-1 and L-2 illustrate two of the screens in the FSP-the front and back sides of an LS-3 SCA. Close inspection of this sample reveals that the following types of problems are illustrated: broken glass, missing glass panel, cracked glass, pad separation, and HCEs with bowing fluorescent tubes and loss of vacuum. Because the field operator has an image of the current 
condition, he/she will enter only new problems. If he/she tries in error to enter a previously logged problem, the software will detect the double entry.

Maintenance, Planning, Operations, and Engineering use the system to examine trends in failures or repair, to check the current status of the fields, and to use a key tool in the biweekly planning meeting on solar field maintenance.

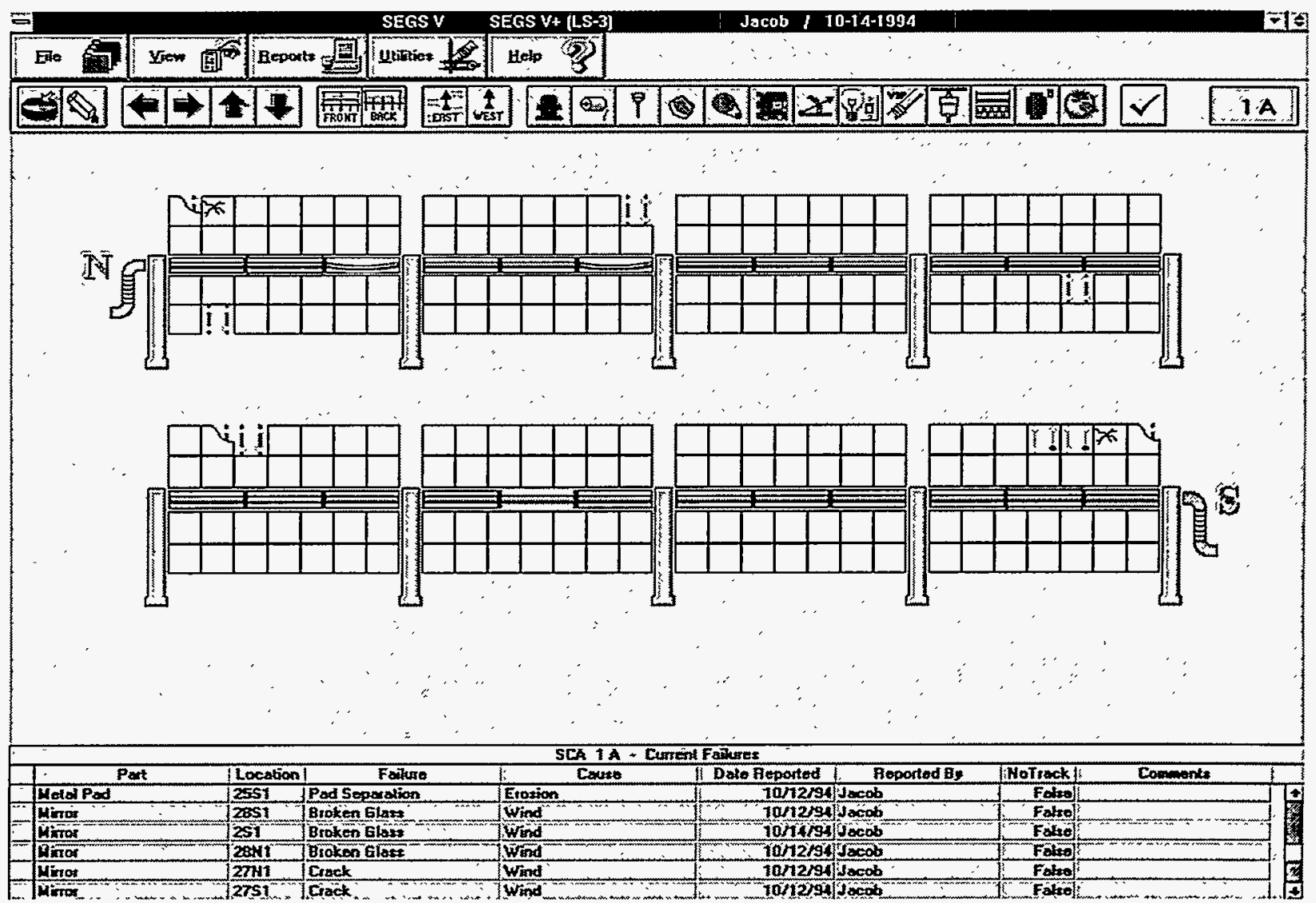

Figure L-1. Front side of the SCA. 


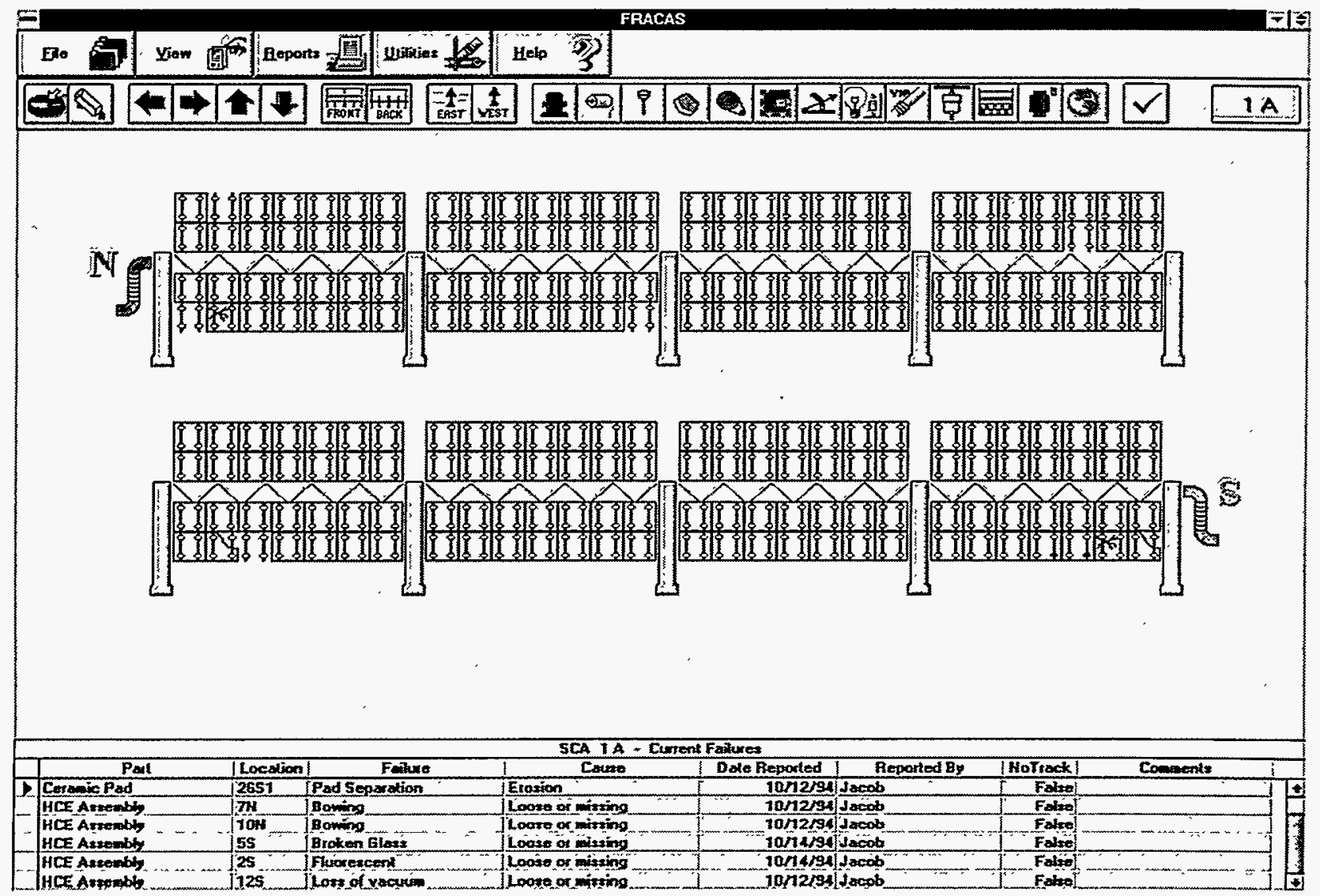

Figure L-2. Back side of the SCA. 
This page intentionally left blank. 


\section{Appendix M \\ REMOTE DATA ENTRY TO \\ SOLAR Field MAINTENANCE DATABase}

Realizing that using the Field Status Program (FSP), described in Appendix L, would be greatly improved by remote entry of data into the database, methods to transmit data from the field operator directly to the network were explored. The selected method consists of a wireless truckmounted unit communicating to a network transport system using a tailored software system.

The FSP remote data entry service is driven by the need of the field technician. Its primary goal is to allow the technician to access database resources while in the field. This goal is achieved through a simple implementation of a wireless network combined with a portable PC-based workstation that is located in the technician's service vehicle. The service vehicle, PC, and technician are collectively known as the "FSP unit." The FSP unit can then monitor, track, and $\log$ the status of all the solar collector assembly (SCA) units in each solar electric generating system (SEGS) while on location. This service is of great value to the operation of each SEGS, because it gives the power plant operator an immediate status of all of the solar field components and allows for planned maintenance without affecting production.

The FSP remote access system itself is implemented using several different technologies that are tightly bundled together. These technologies can be broken down into three separate layers, namely hardware, transport, and software. Each of these layers is described below.

\section{Hardware}

The hardware used for the FSP remote data system ranges from a variety of PC architectures and "cutting-edge" radio technology. The PCs are primarily used as the interface between the technician and the data systems that are housed by the local area network (LAN). The requirements served by the hardware are as follows:

1. Some form of a computer or terminal must be located in the service vehicle to function as an entry point so that the technician can access data resources located on the LAN.

2. Some means of "transport" must exist to allow data to be passed to and from the remote PCs and the database systems. This "medium" can incorporate physical connections via cables or wireless technologies.

All of these requirements must be fulfilled for successful transmission of data to and from the remote $\mathrm{PC}$ and server. Installing a truck-mounted remote computer was not a trivial task. Certain aspects had to be taken into consideration when choosing the equipment and "style" of the mount. The system had to be durable, reliable, compact, and have a long range of communication. The solutions to these needs are described below in detail:

- The client systems located in the service vehicle are designed for durability and compactness. They consist of a 100-MHz Pentium-based PC that is enclosed in a case no larger than 6 inches $\times 4$ inches $\times 2$ inches. The units are to be located behind the driver's seat. 
This is the ideal place for mounting because it will eliminate cab clutter and minimize exposure to direct sunlight.

- The type of display was important, resulting in an 11.3-inch laptop-based LCD display that could be mounted on the driver-side visor. Because the display is less than 2 inches thick and weighs less than a pound, it can be easily folded up and out of the way, yet it is close enough for the driver to get a good view without excessive eye or neck strain. This again should keep the display unit from direct exposure to sunlight when not in use.

- Input devices for the system consist of an industrial-strength membrane keyboard and mouse. These two components are designed for just this type of environment. There are no mounting points for these two devices, because it would severely limit the mobility of the operator. Interface connections for the units will be mounted on the dash to allow a quick connect/disconnect point for the input devices. A rack will be located on the dash so that the mouse and keyboard can be stored in an appropriate place while the truck is in motion and/or the system not in use.

- The transport medium is provided via a wireless radio network known as MavNet. The radio network consists of "Maverick" cells that use radio technology to communicate with a centralized Maverick hub, known as a "Pathfinder." Each vehicle that houses a client PC must also house a Maverick cell. The Pathfinder itself is connected to the LAN that contains the plant's database systems. This makes a data path from each of the client PCs to the server that contains the database systems. Figure M-1 gives a visual perspective of this setup.

- The Maverick units operate from a 12-volt power supply (the truck's power system) and broadcast in the $400-\mathrm{MHz}$ range at 15 watts, which has a range of up to 30 unobstructed miles and 4 to 5 miles with heavy obstructions. Because of the large amount of obstructions located in the plant, frequency must be kept at a low enough level so that an omnidirectional antenna may be used. This is critical to the mobility of the service vehicle. The bandwidth between the radio cells and the pathfinder are subsequently limited to 19.2 KBPS. However, the range of the radio network is as high as 30 miles unobstructed. For applications requiring greater range, a repeater would be required (adding an additional directional 30 miles). In fact, with repeater technology, there is no limit to distance.

- The speed of the system is not altogether limited to 19.2 KBPS. Multiple radios can be bundled together in each station to receive more bandwidth. The bandwidth would be gained in steps of 19.2 KBPS. This would also increase the reliability of the radio connection. If one radio were to lose signal, the other(s) would still keep the connection up and running. Two radios in each unit would give a little more performance than a standard dial-up network connection over conventional telephone lines. This option has been left open for possible future development when the need arises. 


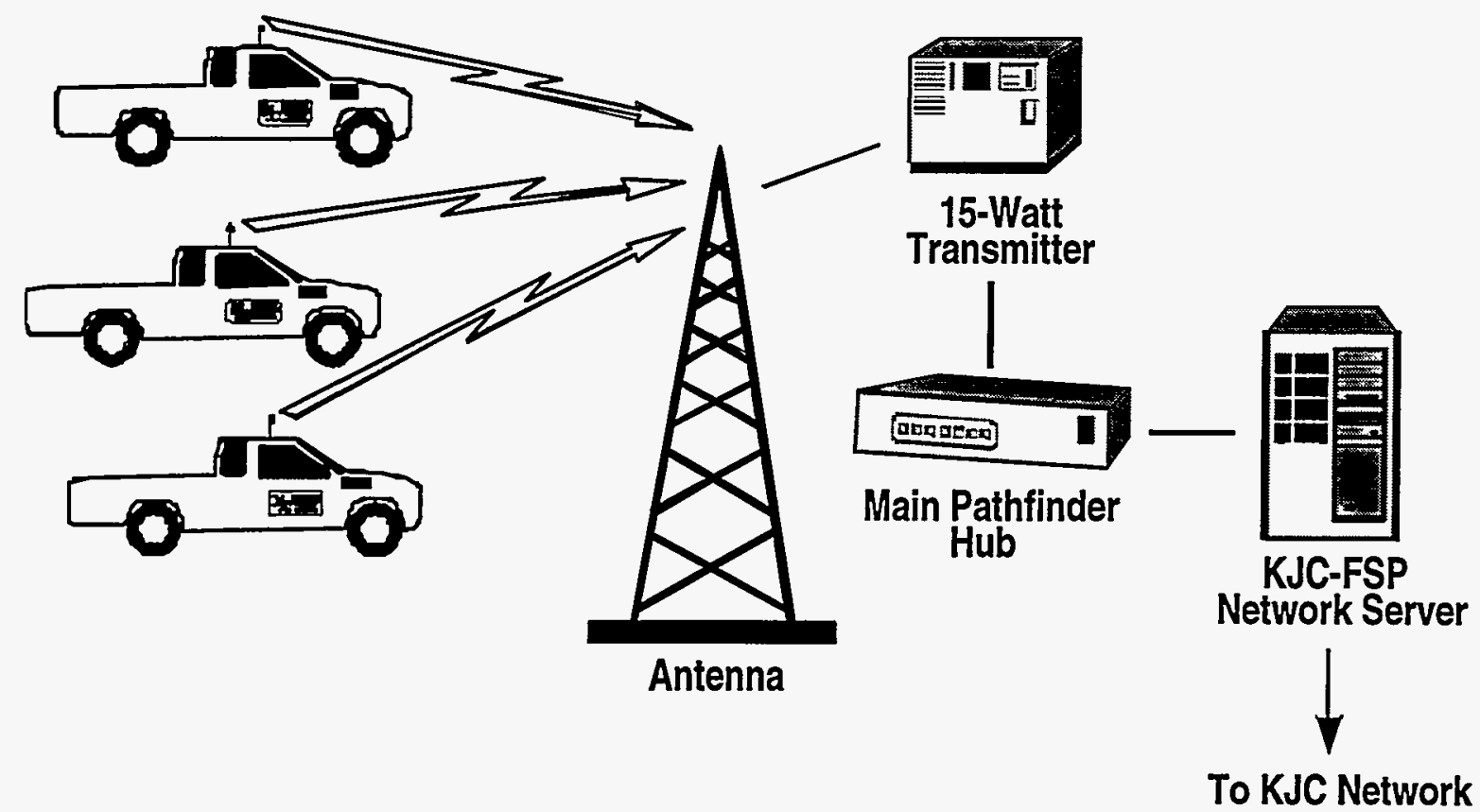

Figure M-1. Remote solar-field data entry at Kramer Junction.

\section{Transport}

In order for the remote systems to communicate, a transport mechanism must be implemented. This is achieved by using the Maverick technology. The Maverick units are the core of the connectivity between the LAN and truck units. MavNet itself uses a particular communication protocol called SLIP (Serial Line IP) to emulate a "wired" connection over a common serial interface. The MavNet collective is a modular component of the system that makes up the "medium" on which network communication will flow. Located on each Maverick unit and the Pathfinder are one or more standard serial ports. The MavNet's configuration allows redirection of data from the serial port of one Maverick unit to another. This is done using standard IP transport and simple routing mechanisms that are inherent with the MavNet units. This configuration has been modified slightly to allow multiple Maverick cells to interface their serial connections to one serial port only on the back of the Pathfinder. Use of SLIP, a SLIP gateway, and custom routes achieve this interface.

The SLIP gateway holds true to its name. A standard PC acts as a "bridge" for network traffic from one medium type to another. Mainly, it interfaces the MavNet with the LAN, thereby completing the data path from client to server. The gateway itself is implemented using the Linux Operating System and SLIP software. This configuration choice was chosen because Linux can be interfaced with almost all standard network topologies and protocols that exist today. The SLIP gateway is then configured to route data between the LAN and the MavNet. From this standpoint, network connectivity is considered stable and complete. 


\section{Software}

Using the above-mentioned network topology, we can treat each remote client as if it were attached directly to the LAN, subject to performance issues. The speed at which MavNet operates is much slower than that of a direct LAN connection. This is the reasoning behind our choice of software.

For efficiency and low-bandwidth reasons, we opted to use Cytrix WinFrame. This client/server package gives multiple clients the capability of using NT server resources to their full potential. The client treats your computer as a virtual terminal for the server. In a sense, all operations are performed on the server, while the client PC is used strictly for input and output of data. This means a minimal amount of data traverses the network medium.

The client software will then be loaded on the PCs that are located in the trucks, while all of the database applications will be loaded onto the WinFrame server. This ensures that the least amount of data will have to traverse the LAN and MavNet. The software also allows the client to restore a "broken" connection. If the host PC loses radio communication, its session will still run on the server. In essence, the server will place the client's session, and all programs that are executing, on hold until the client reconnects. After reconnection, the server will place the client back into the same environment that the client was in before the connection loss. The server logs and tracks all clients that are attached for just this purpose. Furthermore, because of the nature of Cytrix WinFrame, client administration will be kept to a bare minimum, as all remote user programs, data, and interfaces are stored in one central location.

On top of the Cytrix WinFrame, we will use a custom database application that was designed specifically to report, log, and track SCA failures. This program, known as FSP, is the software package that has been used for some time to handle the overwhelming task of keeping failure reports organized and accessible. Of course, the system is not limited to just this program. We can use virtually any software application that will run on an NT 3.51 server.

\section{Closing Comment}

The entire system is modular. Therefore, this approach can be used with virtually any network configuration and still provide network access to a remote PC. This technology could be integrated into several other environments that have remote computing needs. Because of the long range of this system, and its upgrade and enhancement possibilities, it is flexible enough to integrate with most preexisting systems that require an excessive amount of data logging and tracking. Future steps will include the Cytrix WinFrame from the NT 3.51 version to the 4.0 version; Cytrix claims a $20 \%$ increase in application speed per connection. 


\section{Appendix N \\ COMPUTERIZED DRAWING AND DOCUMENT CONTROL}

Upon evaluation of the technical documentation methods at the Kramer Junction, California, power park, including drawings, system and equipment specifications, operating and maintenance procedures, and technical manuals, it was clear that computerized cataloguing, control, and accessibility were necessary to adequately deal with five separate plants and a dispersed maintenance crew. The clear solution was to implement a computer-based system to allow for easy access and retrieval of the most critical information. In such a system, documentation such as drawings can be viewed plant-wide via the site computer network.

\section{Document Control}

Commercial documentation database systems were evaluated for scope, flexibility, and costeffectiveness. The package AutoManager Workflow 3.1 was selected for implementation, and it has since been augmented with View Base so that scanned images (raster images) and AutoCad images (vector images) can be viewed simultaneously. This package has the capability to catalog documentation and to allow remote station retrieval and on-screen review. Suggested changes can be made from the remote station, with final control for documentation changes remaining with a few designated individuals. Implementation has taken place systematically in 1995 , including the preparation of essential documentation for entry into the system. Targeted for entry are major piping and instrument drawings (P\&IDs), overall site plans, specifications, operating procedures, and technical manuals. Approximately 20 P\&IDs were selected from Solar Electric Generating Systems (SEGS) VI through VII, and a total of 160 P\&IDs for all the plants. As with many tasks, the development of the process and implementation on selected plants was carried out under the Sandia National Laboratories/KJC Operating Company ( $\mathrm{SNL} / \mathrm{KJCOC}$ ) cost-shared program, and then full implementation is accomplished under the normal KJCOC operation and maintenance budget.

For drawings, the identification variable with examples includes the following:

- Plant - SEGS III or V

- System - turbine, cooling water, solar field

- Document Type - piping, mechanical, or electrical drawing

- Drawing Number - assigned by KJCOC

- Original Drawing Number - assigned in design phase, e.g., by LUZ International Ltd.

- Vendor $-\mathrm{ABB}$ or Westinghouse

Figure $\mathrm{N}-1$ shows the customized screen that was prepared for control of document information access. 


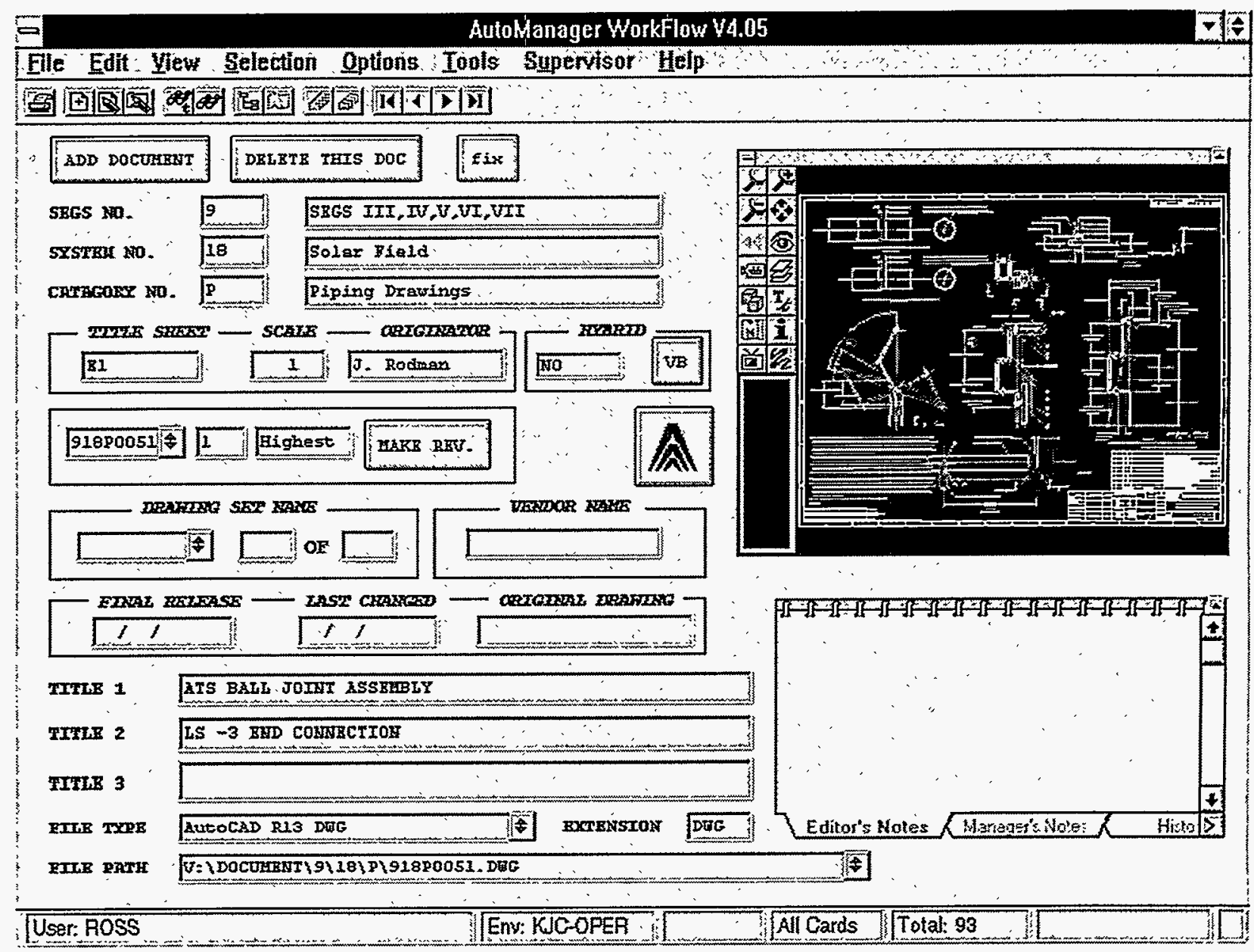

Figure N-1. Customized screen for control of documentation information access.

\section{Computerization of Improved Solar Field Maintenance Procedures}

A preliminary manual has been prepared containing 19 improved routine maintenance procedures for the solar field. Previous procedures lacked detail and specificity, a section of safety concerns, and illustrated instructions. Furthermore, new techniques and maintenance methods necessitated revisions of many procedures.

Each new procedure includes a component identification; a list of the required tools, materials, and safety equipment; an illustrated explanation of the maintenance procedure; and a selfadministered examination on the procedure to test understanding. Figure N-2 shows a sample illustration of a heat transfer fluid (HTF) loop used in the procedure for evacuation of HTF (which is required, for example, when heat collection elements are replaced). At the bottom of the figure are more detailed illustrations of header valve assemblies at the end of each loop. Besides a printed version, users can access a read-only CD-ROM via the plant computer network. 


\section{HEAT TRANSFER FLUID LOOP EVACUATION (LEVEL 2) METHOD 1}

\section{Component Identification}

\section{General Overview}

1. HTF flow from power block (culd)

2. "Inlet" block valve

3. Main line for "inlet" flow into loop

4. Flow control valve

5. "Inlet" drain valve (evac/vent area)
6. Torque/truss assembly

7. Mirror (non-reffective side of SCA)

8. Center of loop

9. "Crossover" pipe assembly

10. "Crossover" drain valye (air/vent area)
11. "Outlet" drain yalve (evac/vent area)

12. "Outlet" block valve

13. Main line for "outlet" flow from loop

14. HIF now to power block (hot)
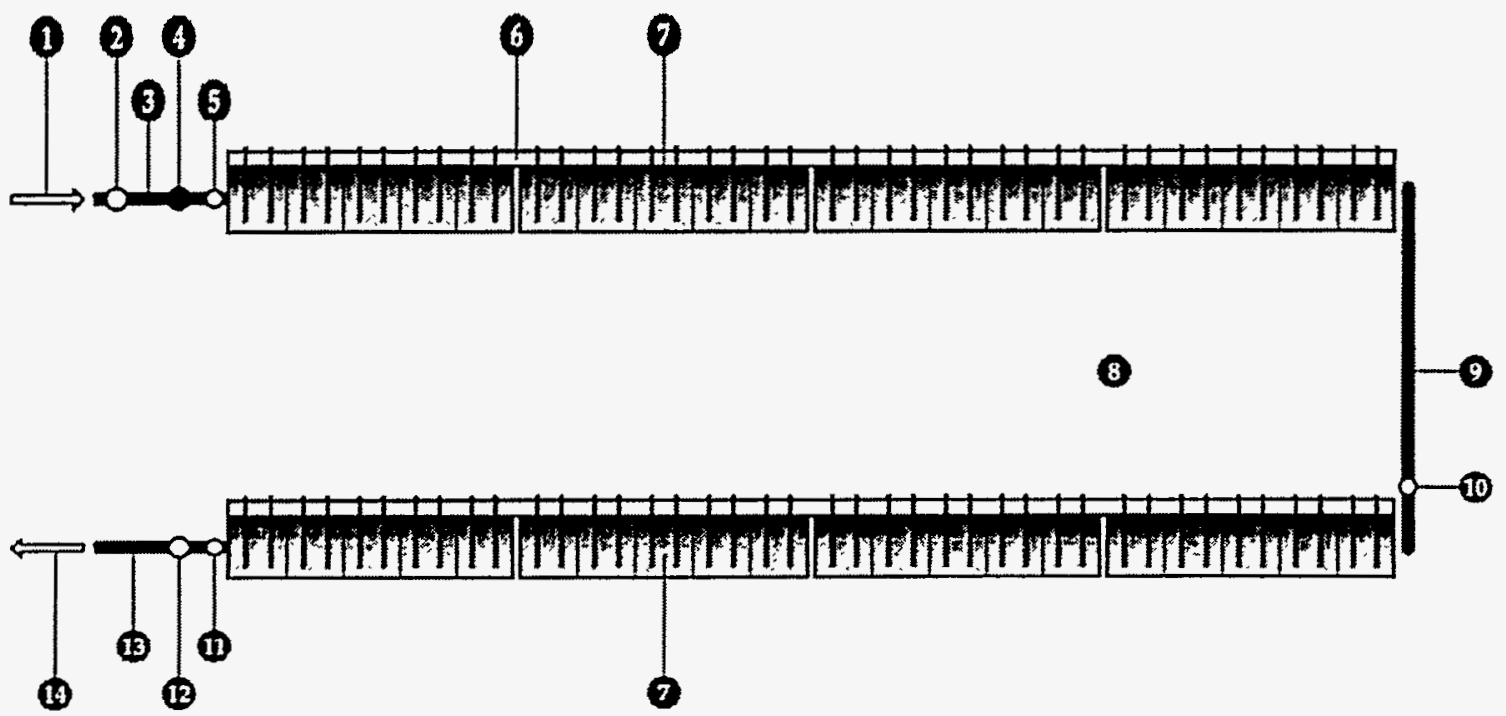

Detailed Header Valve Assemblies Overview

"Inlet" location

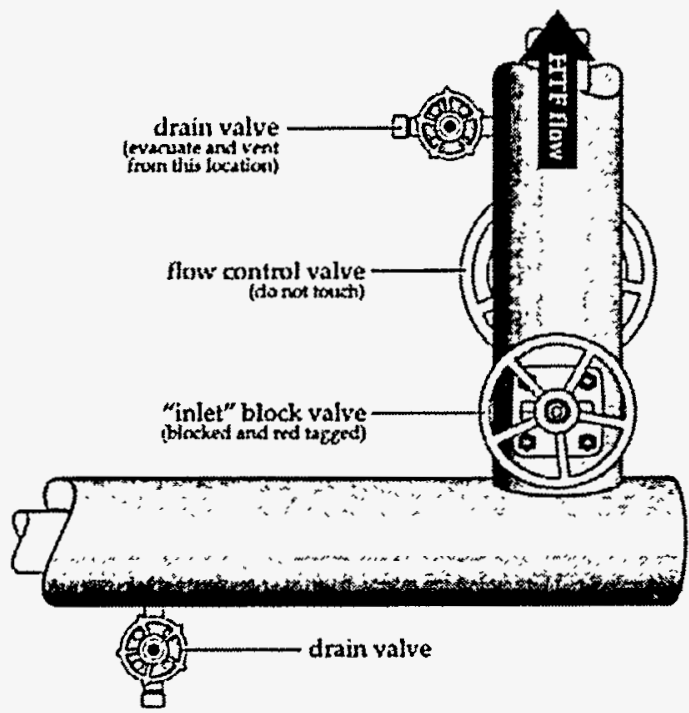

\section{"Outlet" location}

pressure relief valve

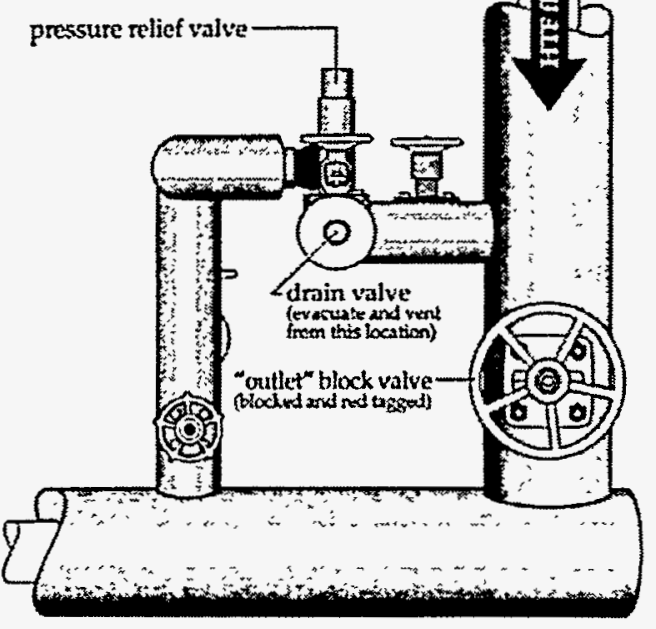

Figure N-2. An HTF loop used in the procedure for evacuation of HTF. 
Below is a list of the table of contents of the Maintenance Manual showing the procedures prepared to date. While the concept, plan, and initial procedures for the manual were developed under the cost-shared SNL/KJCOC program, many of the procedures shown were subsequently written as part of the normal operation and maintenance budget.

- LS-2 and LS-3 Heat Collection Element Replacement

- Heat Transfer Fluid Evacuation (Level 2) Method 1

- LS-2 and LS-3 Weld Leak Repairs

- LS-2 Double Flex Hose/Ball Joint Replacement

- LS-2 Single Flex Hose/Ball Joint Replacement

- LS-3 Gear Box Replacement

- LS-3 Truss Alignment

- LS-3 Drum Lube

- LS-2 and LS-3 Mirror Replacement

- LS-2 and LS-3 Pad Failure Repair

- LS-2 Pad Reinforcement Disc Installation

- LS-3 Motor and Brake Assembly Installation

- LS-2 Sun Sensor Alignment

- LS-3 Sun Sensor Alignment

- LS-2 Logic Board Replacement

- LS-3 Logic Board Replacement

- LS-3 Motor Board Replacement

- LS Motor Replacement

- SEGS Component Identification 


\section{Appendix 0 \\ COMPUTER-BASED OPERATOR LOG}

\section{Background}

As part of the routine monitoring of the solar electric generating system (SEGS) projects, data on electric production, gas and electric consumption, solar field thermal production, and solar field and plant availabilities are essential for normal day-to-day operation planning and long-term project tracking.

Historically, one of the primary responsibilities of the Performance Engineering department was to collect daily handwritten operator logs and enter this data into a computer database for historical tracking and general monitoring of the plants. The operator logs were collected daily from each of the SEGS projects and keyed into a database by clerical staff. Performance Engineering reviewed the data and added process data collected from the instrument controls interface (ICI) or distributed control system (DCS) plant process computer systems. Delays in data input were common because of the availability of clerical staff and the time required to collect and process plant data. Data entry errors were common because the data entry person did not have any particular feel for or understanding of the data being entered.

Initially, Performance Engineering generated daily, monthly, and annual reports from this data; however, because of staff reduction, daily reports were eliminated. As a result, Operations generated its own daily report to summarize the plant's performance for the daily morning meeting. The night operator often spent a substantial portion of time generating the handwritten daily operations summary report. Some of the desired calculations were too complex to be handgenerated by the operators; thus, Operations either estimated the values or did not provide them. In addition, the Operations supervisors arrived early each morning to collect the logs from each of their plants and review and correct the reports before the morning operation and maintenance (O\&M) meeting.

\section{Scope}

The purpose of this task was to develop a new KJC Operating Company (KJCOC) proprietary performance-database software to be implemented on the new site computer network (see Appendix 1). This software would allow Operations staff to enter the log information each night into the database from their workstation in the control room. Thus, each morning the database would be updated with the previous day's data, allowing the Operations supervisor to review and generate the previous day's report from his/her computer. Because the report is computergenerated, additional information will be available, which will allow better O\&M decisions to be made. In addition, the Operations Department will have direct access to historical data, which in the past was available only via contact with Performance Engineering. Upon considering these goals, it was decided to develop a proprietary software program, OpLog ${ }^{\circledR}$, to meet this need. 


\section{Development of OpLog ${ }^{\odot}$}

\section{Specifications}

A detailed program specification (listed below) was developed that identified the objectives of the computer program, the data to be stored in the database, calculations to be performed, reports to be generated, and expected future expansion requirements.

1. Develop a new performance database to reside on the site network.

2. Eliminate the need for separate clerical data entry by allowing Operations to enter data during the day and complete it during the night shift. This type of entry will save operators' time on manual hand calculations and will save the Operations supervisor's time collecting and reviewing reports. It will also improve timeliness of data because it will be entered on a daily basis, which will allow the data to be used for daily operational decisions.

3. Program will generate reports for Operations and Performance Engineering. The program will generate Daily Operations Summary Reports, Summer On-Peak/Mid-Peak Operation Reports, and Monthly Performance Reports.

4. Program will provide increased access to data via the network. The program can be accessed from any PC on the site network and will reduce the need for paper-generated reports, but will allow anyone to generate a paper report.

5. Improve automated error checking of data entry with provision for immediate on-line calculation as data is entered.

6. Program will have two built in audit levels to protect data integrity. Security access levels will be used to limit the ability of users to change data once it has been audited.

7. Simplify tracking of out-of-service equipment and operator comments.

8. Provide a user-friendly interface for program.

9. Future expansion will allow for additional points and automatic data transfer from plant process computers (field supervisory controller and ICIDCS).

\section{Select Database Software}

A large number of database software options are available. It was decided that a Windows-based system would probably allow for the quickest development time, best possible user interfaces, and the most flexibility and functionality. Three options were considered: Paradox for Windows, Fox Pro for Windows, and Visual Basic with add-in database tools. Visual Basic was excluded because it was thought that a database program would allow for easier access to the data in the future. Paradox for Windows was selected over Fox Pro for Windows because of its Windows implementation. Paradox allows data to be stored in either Paradox or dBase format. In addition, Paradox has close links to Quattro Pro for Windows, which is the Windows spreadsheet selected for use by the company. For future implementations, a software developer should also consider using Visual Basic, $\mathrm{C}++$, Java, or Delphi. 


\section{Identify Data to be Stored in the Database}

The data to be included in the database were identified. The data includes the data previously recorded in the Performance Engineering Database and on the Operator's Daily Operational Report.

\section{Develop Computer Program}

The OpLog $\left.{ }^{(}\right)$computer program was written by KJCOC. The program was set up to function as a stand-alone program that includes all the functionality of the end product except for multiuserrelated issues.

Following the completion of the initial program, it was installed at the site for review and testing. A list of problems and requested changes were developed. This activity was to ensure that the program met the needs of all intended end users. A final version was developed and installed on the network in 1994. Figures O-1 and O-2 show a version of the daily report and daily summary for SEGS III through VII.

\section{Results and Conclusions}

The networked-centralized $\mathrm{OpLog}^{\odot}$ database has proven to be very valuable for maximizing performance and optimizing operational flexibility of the SEGS plants at Kramer Junction, California. A reduction in support staff and improvements in the efficiency of operations personnel have resulted from the installation of this networked centralized database.

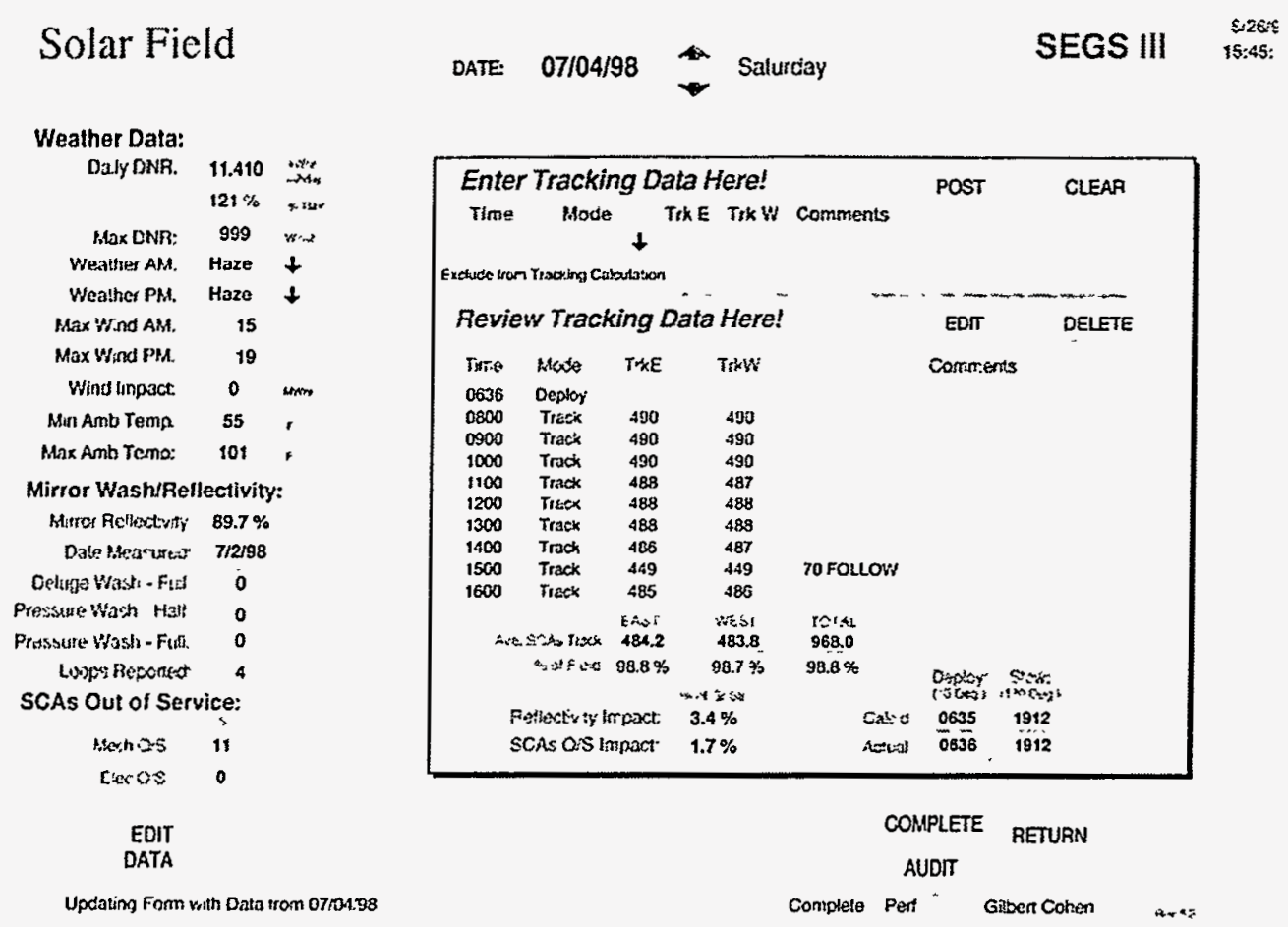

Figure 0-1. Sample daily report $\log ^{*}$ for SEGS III.

\footnotetext{
"Copyrighted by KJC Operating Company.
} 


\begin{tabular}{|c|c|c|c|c|c|c|}
\hline SEGS I & 111 & $\mathrm{Y}$ & M & RY & & nesday \\
\hline SEGS & III & IV & $V$ & $V_{1}$ & VII & III - VII \\
\hline $\begin{array}{l}\text { Solar Field } \\
\text { Tolal Dally DNR - kWh/m2/day } \\
\% \text { of TMY } \\
\text { Max DNR - Wimz }\end{array}$ & $\begin{array}{l}11971 \\
1270 \% \\
1.009\end{array}$ & $\begin{array}{l}11.971 \\
1270 \% \\
1.009\end{array}$ & $\begin{array}{l}11971 \\
1270 \% \\
1.009\end{array}$ & $\begin{array}{l}11971 \\
1270 \% \\
1,009\end{array}$ & $\begin{array}{l}11971 \\
1270 \% \\
1.009\end{array}$ & $\begin{array}{l}11971 \\
1270 \% \\
1.009\end{array}$ \\
\hline $\begin{array}{l}\text { Tracking - \% } \\
\text { SCAS Not Avatlatle - म̈ } \\
\text { SCAS Cleared - Full SCAS } \\
\text { Reflectwity - \% } \\
\text { Solar Field Thermal - MMBtu }\end{array}$ & $\begin{array}{c}999 \% \\
0 \\
238 \\
905 \% \\
5.071 \\
\end{array}$ & $\begin{array}{c}997 \% \\
3 \\
476 \\
90.9 \% \\
5,022 \\
\end{array}$ & $\begin{array}{c}99.8 \% \\
110 \\
0 \\
90.6 \% \\
5.972 \\
\end{array}$ & $\begin{array}{c}999 \% \\
0 \\
0 \\
906 \% \\
4,256 \\
\end{array}$ & $\begin{array}{l}989 \% \\
110 \\
768 \\
89.9 \% \\
4.383 \\
\end{array}$ & $\begin{array}{l}996 \% \\
1482 \\
905 \% \\
24,704 \\
\end{array}$ \\
\hline $\begin{array}{l}\text { Gross Electric Production } \\
\text { Mas Solar-MWe }\end{array}$ & 390 & 390 & 420 & 385 & 382 & 420 \\
\hline Max Boller - MWe & 00 & 00 & 300 & 00 & 00 & 300 \\
\hline Gross Solar - WWh & 408 & 406 & 435 & 411 & $\begin{array}{r}391 \\
0\end{array}$ & $\begin{array}{r}2.051 \\
101\end{array}$ \\
\hline $\begin{array}{l}\text { Gross Boller - MWh } \\
\text { Tolal Gross - MWh }\end{array}$ & $\begin{array}{r}0 \\
408\end{array}$ & $\begin{array}{r}0 \\
406\end{array}$ & $\begin{array}{l}101 \\
536\end{array}$ & $\begin{array}{r}0 \\
411\end{array}$ & $\begin{array}{r}0 \\
391\end{array}$ & $\begin{array}{r}101 \\
2,152\end{array}$ \\
\hline $\begin{array}{l}\text { Net Electricity Sold - A Chan } \\
\text { On-Pk - MWh } \\
\text { Mid-Pk - MWh } \\
\text { Of-Pk - MWh } \\
\text { Super Off - MWh } \\
\text { Total - MWh }\end{array}$ & $\begin{array}{r}204.1 \\
1571 \\
9.4 \\
370.7\end{array}$ & $\begin{array}{r}203.0 \\
1546 \\
95 \\
\\
367.1\end{array}$ & $\begin{array}{r}2138 \\
2544 \\
108 \\
479.0\end{array}$ & $\begin{array}{r}1954 \\
1561 \\
93 \\
360.8\end{array}$ & $\begin{array}{r}1836 \\
1502 \\
89 \\
\\
342.7\end{array}$ & $\begin{array}{r}9999 \\
872.5 \\
479 \\
00 \\
1920.3\end{array}$ \\
\hline $\begin{array}{l}\text { Net Purchase - B Chan } \\
\text { On-Pk - MWh } \\
\text { Mic-Pk - MWh } \\
\text { Off-Pk - MWh } \\
\text { Total - MWh } \\
\end{array}$ & $\begin{array}{l}00 \\
13 \\
30 \\
4.3 \\
\end{array}$ & $\begin{array}{l}0.0 \\
1.6 \\
3.2 \\
4.8\end{array}$ & 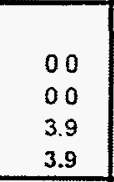 & $\begin{array}{l}00 \\
15 \\
41 \\
5.6\end{array}$ & $\begin{array}{l}00 \\
15 \\
40 \\
5.5\end{array}$ & $\begin{array}{r}00 \\
58 \\
182 \\
24.0 \\
\end{array}$ \\
\hline $\begin{array}{l}\text { Station Load } \\
\text { Station Internal - MWh } \\
\text { Station External (B Chan) - MWh } \\
\text { Total Station - MWh } \\
\text { Station Internal - } \% \text { of Gross } \\
\end{array}$ & $\begin{array}{r}373 \\
43 \\
41.6 \\
91 \% \\
\end{array}$ & $\begin{array}{r}38.9 \\
48 \\
43.7 \\
96 \% \\
\end{array}$ & $\begin{array}{r}57.0 \\
39 \\
60.9 \\
106 \% \\
\end{array}$ & $\begin{array}{c}502 \\
56 \\
55.8 \\
122 \% \\
\end{array}$ & $\begin{array}{r}483 \\
55 \\
53.8 \\
124 \% \\
\end{array}$ & $\begin{array}{r}231 ? \\
240 \\
255.7 \\
108 \% \\
\end{array}$ \\
\hline $\begin{array}{l}\text { SCE Capacity Factor } \\
\text { On-Pk }-\% \\
\text { Mid-Pk }-\% \\
\text { Off-Pk }-\% \\
\end{array}$ & $\begin{array}{r}134 \% \\
582 \% \\
35 \% \\
\end{array}$ & $\begin{array}{r}1128 \% \\
57.3 \% \\
3.5 \% \\
\end{array}$ & $\begin{array}{r}1188 \% \\
94.2 \% \\
4.0 \% \\
\end{array}$ & $\begin{array}{r}1086 \% \\
57.8 \% \\
3.5 \% \\
\end{array}$ & $\begin{array}{r}1020 \% \\
556 \% \\
3.3 \% \\
\end{array}$ & $\begin{array}{r}1111 \% \\
646 \% \\
35 \% \\
\end{array}$ \\
\hline $\begin{array}{l}\text { SCE Scheduled Mainten. } \\
\text { On-Pk - hrs } \\
\text { Mrd-Pk - hrs } \\
\text { Oif-Pk - hrs }\end{array}$ & & $\begin{array}{l}0 \\
0 \\
0 \\
\end{array}$ & $\begin{array}{l}0 \\
0 \\
0\end{array}$ & $\begin{array}{l}0 \\
0 \\
0 \\
\end{array}$ & $\begin{array}{l}0 \\
0 \\
0 \\
\end{array}$ & $\begin{array}{l}0 \\
0 \\
0 \\
\end{array}$ \\
\hline $\begin{array}{l}\text { Natural Gas Use } \\
\text { Botler - KSCF } \\
\text { Botler Stant-up - KSCF } \\
\text { Aux Heater - KSCF } \\
\text { Total - KSCF } \\
\end{array}$ & $\begin{array}{l}0 \\
0 \\
0 \\
0 \\
\end{array}$ & $\begin{array}{l}4 \\
0 \\
0 \\
4 \\
\end{array}$ & $\begin{array}{r}1,224 \\
38 \\
0 \\
1,262 \\
\end{array}$ & $\begin{array}{l}0 \\
0 \\
0 \\
0 \\
\end{array}$ & $\begin{array}{l}0 \\
0 \\
0 \\
0 \\
\end{array}$ & $\begin{array}{r}1,228 \\
38 \\
0 \\
1,266 \\
\end{array}$ \\
\hline $\begin{array}{l}\text { Solar Mode Heatrate - MMBtu/MWh } \\
\text { Boler Mode Gasrate - KSCF/MWh }\end{array}$ & $\begin{array}{l}124 \\
\cdots\end{array}$ & 12.4 & $\begin{array}{l}137 \\
121\end{array}$ & $\begin{array}{l}104 \\
--. \\
\end{array}$ & $\begin{array}{l}11.2 \\
-\cdots\end{array}$ & $\begin{array}{l}120 \\
121 \\
\end{array}$ \\
\hline
\end{tabular}

Figure 0-2. Sample daily report summary" for SEGS III-VII.

\footnotetext{
"* Copyrighted by KJC Operating Company.
} 


\section{Appendix P Heat Transfer Fluid Pump Upgrade}

\section{Background}

Heat transfer fluid (HTF) pump seal failures have been a continuing problem at the Kramer Junction, California, solar electric generating system (SEGS) plants, imposing excessive demands on labor and spare parts for this single component. Over a 12-month period in 1992 to 1993, the mechanical seal on the SEGS V B pump failed 42 times at a total cost of about $\$ 250,000$. The problem was exacerbated by failures in the summer period when the pumps are run at full rating, because this is the period when electricity revenues are peak and pump failures can result in high lost revenues.

The high failure rate was associated with a single-bellows mechanical seal, and was believed to be caused by many factors, e.g., casing stresses due to piping misalignment, high seal operating temperature, and excessive pump vibration due to a variety of causes, such as cavitation and vibrations transmitted from the motor via the motor-pump coupling.

\section{Scope and Objectives}

The overall objective of this task was to find a reliable and cost-effective solution to the seal problem on the HTF pumps. It was decided to attack the problem from several sides. Seal failures on this pump are primarily a result of the service (high temperature, moderate pressure) and pump vibration. Initial evaluation indicated that steps should be taken to reduce vibration along with the selection of an adequate seal.

\section{Results}

In 1992, an attempt was made to replace the original seal with a simple ceramic packing on a modified shaft. This solution failed, and new directions were explored. In addition to the seal, modifications to the piping, thrust bearing, pump-motor coupling and seal cooling system were investigated. As a result, the thrust bearing was upgraded from a $7310 \mathrm{MRC}$ bearing to a 8310 $\mathrm{MRC}$ bearing to improve reliability; the new bearing is designed for higher loading and has improved thermal stability. A flexible coupling was then installed to replace the original rigid coupling in order to reduce vibration on the pump from the motor.

In 1993, a new type of HTF seal was installed on the SEGS V B HTF pump. The seal is a single-coil spring Type D balanced mechanical seal, designed for higher pressure and temperature and offering expectations of better sealing and increased durability.

In parallel with the seal installation, a heat exchanger was added to the pump coolant loop to maintain the seal at a lower operating temperature, and the expansion tank pressure was raised from 125 psig to 145 psig to increase the pump net positive suction head. In addition, the pressure balance between the series $\mathrm{A}$ and $\mathrm{B}$ pumps was improved.

Because of these changes, pump cavitation was virtually eliminated and the new seal performed without failure for more than one year. It is believed that this excellent performance results from effects of all changes instituted within this task. This fix was implemented on other HTF pumps 
at KJC Operating Company in later years. There are two pumps each at SEGS III, IV, and V, and three pumps each at SEGS VI and VII. The dramatic effect these changes had on the seal failure rate for the entire KJC Operating Company site is shown in Table P-1.

Table P-1. Seal Failure Rate for the Entire KJC Operating Company Site

\begin{tabular}{|c|c|}
\hline Year & $\begin{array}{c}\text { Number of } \\
\text { Seal Failures }\end{array}$ \\
\hline 1990 & 87 \\
\hline 1991 & 104 \\
\hline 1992 & 67 \\
\hline 1993 & 61 \\
\hline 1994 & 39 \\
\hline 1995 & 13 \\
\hline 1996 & 5 \\
\hline 1997 & 1 \\
\hline 1998 & 5 \\
\hline
\end{tabular}




\section{Appendix Q \\ Comparative Testing of Flexible Hoses VERSUS ROTATING JOINTS}

\section{Background}

The heat collection elements (HCEs) in the solar collector assemblies (SCAs) carry the heat transfer fluid (HTF). Within an SCA, the HCEs are welded to each other. The connections of the HTF flow path between adjacent SCAs or between the header and the end SCA are normally made with flexible hoses that allow movement between fixed and rotating parts. The flexible hoses (abbreviated as "flex" hoses hereafter) are made up of a corrugated steel inner tube that is enclosed in stainless steel mesh, covered with insulation, and further enclosed in an outer corrugated tube.

Reliability of the flex hoses has been marginal in the Kramer Junction, California, plants, and it is known that the pressure drops are high because of the inner corrugations and the bends inherent in the flex hoses in different positions of the collector.

The Operation and Maintenance Improvement Program (O\&MIP) evaluated the replacement of the flex hoses with a series of rotating.joints, one at each point of movement. Rotating joints offer the potential of higher reliability, lower investment costs, and lower parasitic power requirements because of a reduced pressure loss. KJC Operating Company (KJCOC) developed a ball-joint assembly outside the O\&MIP. However, the O\&MIP was responsible for performing tests that quantified the reliability and pressure-loss reduction potential of the rotating-ball-joint assembly.

\section{Pressure Loss Test}

The objective of the test was to compare the pressure losses in a test loop with flex hoses compared to an adjacent test loop modified by the installation of rotating joints. The test loops were located in rows 3 through 6 in the northwest quadrant of Solar Electric Generating System (SEGS) VI. In concept, the test loops are similar to the schematic shown in Appendix G, Figure G-1. However, the SEGS VI test loops, which contain LS-2 collectors, have 8 SCAs per row (16 per loop). Flow was measured with vortex shedding meters. In addition to the flow and temperature instrumentation, transducers are located at the inlet and outlet to measure pressure levels at each point (not differential pressure). All instrumentation was tied into a centrally located Campbell data logger. Data were measured each second and recorded as two-minute averages.

\section{Pressure Loss Test Results}

Figure Q-1 compares the pressure drop across a single loop as a function of volumetric flow rate for rotating joints and flex hoses. This data indicate a consistent reduction in pressure drop of about $45 \%$ with the use of ball joints.

An additional comment can be made about the flow measurement in these test loops. The vortex shedding meters were consistent and reliable. Before their use, turbine meters were used but 
their reliability was poor because of the interference of hydrocarbon deposits with bearing operation (see Appendix R).

Ball joints offer a viable replacement for flexible hoses, with the potential for a significant reduction in the HTF pressure losses across the solar field. Some simple calculations will indicate the magnitude of the reduction. The annual capacity factor of a SEGS plant is about $25 \%$. Thus, for a $30-\mathrm{MW}$ turbine, the annual output is about $65,700 \mathrm{MWh}$ per year. The average daily operating hours are about 9 , for a total of 3,285 hours per year. By these estimates, the average turbine load is $20 \mathrm{MW}(65,700 / 3,285$; also obtainable from [ $24 \times 0.25] / 9 \times 30)$. The HTF flow at full capacity is 160 gallons per minute per loop, and for $20 \mathrm{MW}$ the flow is about 100 gallons per minute. The reduction in pressure drop at this flow is due to the use of rotating joints is about 75 psi. This corresponds to a reduction in plant parasitics of approximately $860,000 \mathrm{kWh}$ per year if all the flex hoses were replaced. At an average value of electricity of $10 \not / \mathrm{kWh}$, the annual savings will be about $\$ 86,000$ per plant, or about $\$ 430,000$ for the Kramer Junction site.

\section{Ball Joint vs. Flex Hose Pressure Drop Cumulative Results}

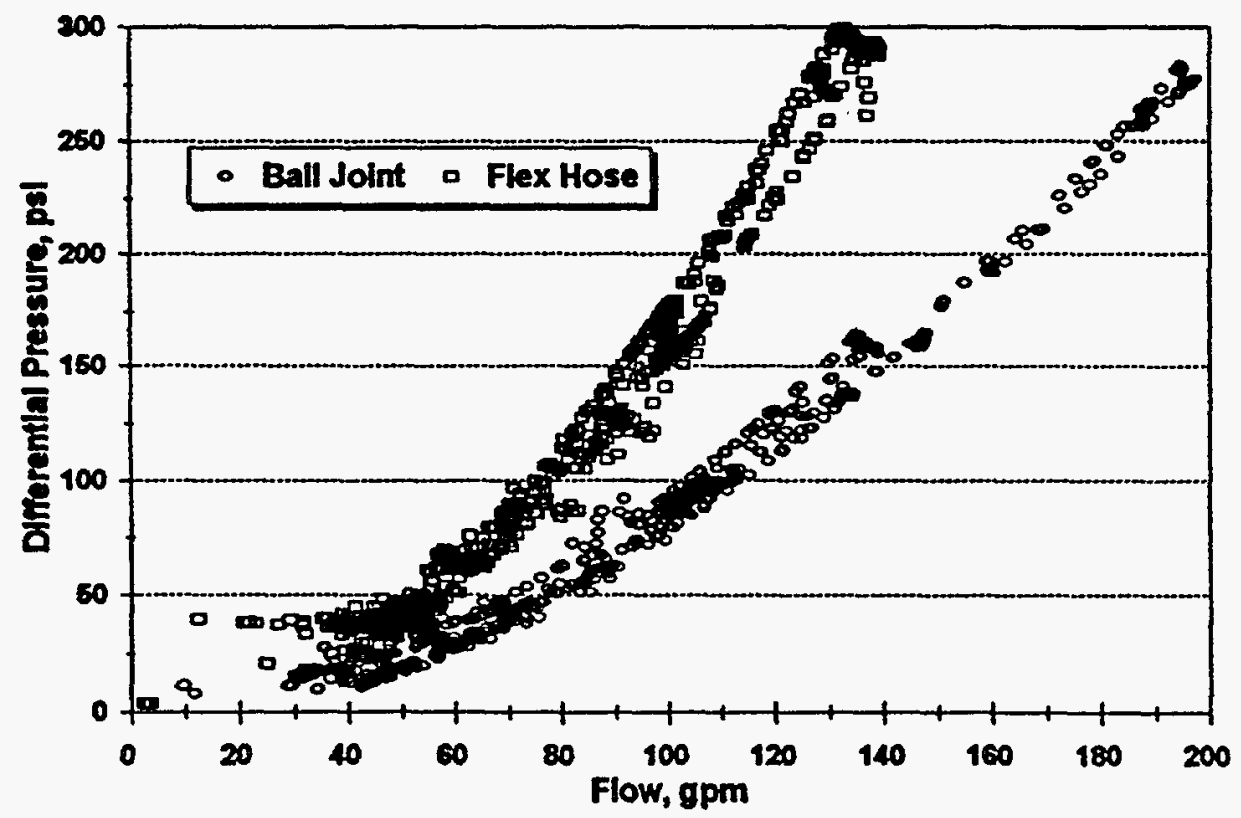

Figure Q-1. Pressure drop data for ball joints and flexible hoses at various flows.

\section{Reliability Test}

A test rig, shown in Figure Q-2, was designed to test fatigue life by providing continuous cycling of two ball-joint assemblies, each a single assembly (used between an SCA and a header). The test was conducted at the temperature and pressure conditions of ball joints in normal operation in a solar field. Located in the southwest quadrant of SEGS VII, HTF entered the test rig from the solar field cold header and was discharged to the hot header. Average fluid temperature was about $500^{\circ} \mathrm{F}$, and average pressure about $190 \mathrm{psig}$. Average pressure drop across the ball joint 
was 13 psig. The cycling rate was about 70 times normal cycling, with three rotating cycles completed each hour. Therefore, 30 years of operation would be simulated in approximately five months.

The test started in early February 1996. Observations were made on leakages of vapor and/or fluid, mechanical failure, and requirements for application of graphite grease for lubrication and sealing.

\section{Reliability Test Results}

Minor vapor emissions were observed in the first set of installed ball-joint assemblies, which otherwise operated satisfactorily for 11,500 cycles, equivalent to 31 years and 6 months. A second set of assemblies were installed in February 1997 and operated for 3,659 cycles, equivalent to about 10 years. Again, only minor vapor emissions occurred in these units.

At this point, operating problems developed in the test rig (not the ball-joint assemblies) and testing was halted because sufficient reliability information had been gathered.

In summary, no catastrophic failures occurred in either set, leading to a high confidence in this approach and a policy to use ball-joint assemblies as future replacements for failed flex hoses at the Kramer Junction site.

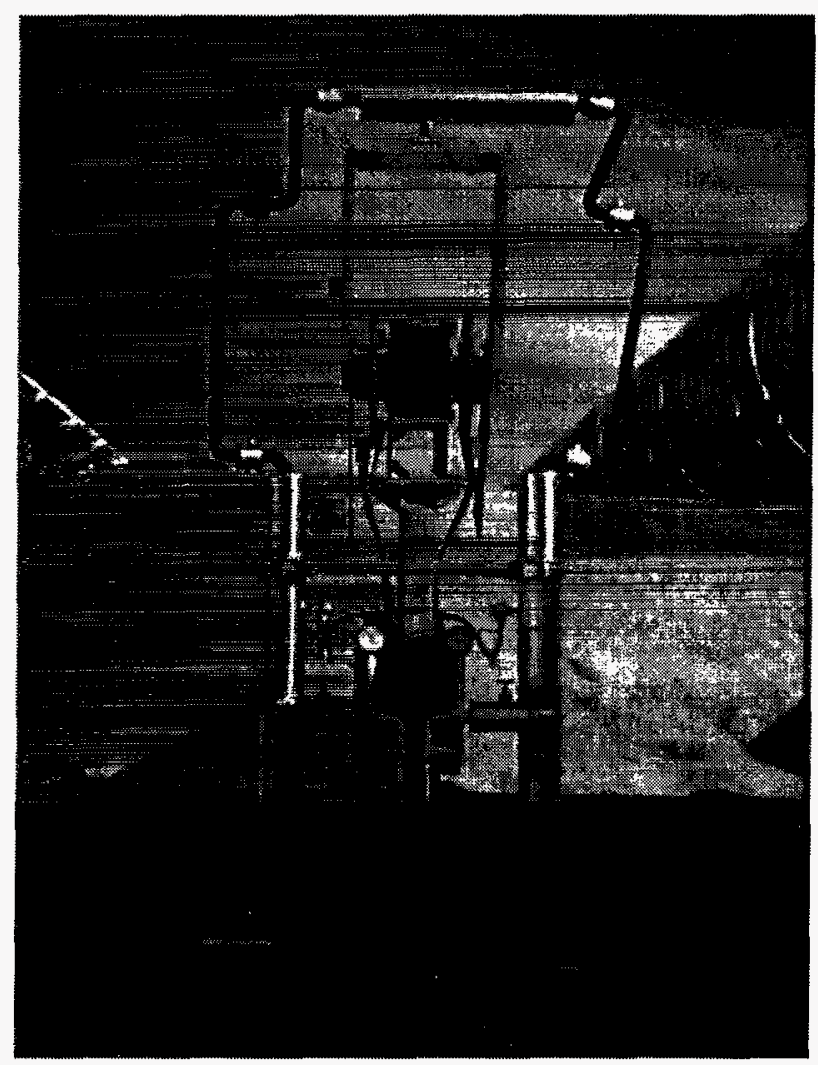

Figure Q-2. Test rig in SEGS VII for the mean time between failures test for ball-joint assemblies. 
This page intentionally left blank. 


\section{Appendix R Heat Transfer FLuid Flow MeAsurement}

\section{Background}

The solar electric generating system (SEGS) solar power plants use a heat transfer fluid (HTF) for collection of solar energy and conversion of the solar heat to steam through an oil/water heat exchange system. During normal solar mode operation, the HTF is pumped with high capacity pumps at the cold $\mathrm{HTF}$ temperature $\left(480^{\circ} \mathrm{F}\right.$ or $550^{\circ} \mathrm{F}$, depending on the plant) through a headerpiping network to the solar field. The HTF is heated to its hot temperature $\left(660^{\circ} \mathrm{F}\right.$ or $\left.735^{\circ} \mathrm{F}\right)$ as it passes through the solar field and is returned to the power block through a second header-piping network.

There are four main reasons that HTF flow measurement is important. First, the measurement directly affects the amount of gas that the plant is allowed to burn. Second, an accurate indication is necessary for operations to optimize solar mode electric production. Third, flow measurement is required to automate the control of HTF flow to the solar field. (Currently, flow is manually controlled by operators.) Finally, an accurate indication of HTF flow is essential for performance analysis on the solar fields and power plants. For example, it is virtually impossible to identify whether a performance shortfall of a few percent is in the solar field or the power block without knowing the HTF flow rate.

Previously, four types of flow meters were used at SEGS plants with varying levels of success: annubar type, insertion turbine, orifice, and low-loss flow nozzles. Each type of flow meter had advantages over the others, but none proved to provide accurate and reliable readings in a consistent manner over time. This may be because of a combination of many factors, such as impurities in the HTF, lack of appropriate preventive maintenance, physical damage to the flow meters, improper installation, improper flow calculation, improper temperature correction, the high temperature of normal operation, freezing of dead leg fluid on differential pressure flow meters, or lack of spare parts.

At the Kramer Junction, California, SEGS plants, the HTF flow rate is measured at three locations. The cold HTF inlet flow rate is measured separately to the east and west halves of the solar field and the hot HTF flow rate is measured after the oil from the two halves has been combined. Initially, insertion turbine flow meters were installed at each of these locations at each plant. In addition to the insertion turbine flow meters, SEGS III through V also had annubar meters at each location. SEGS VI through VII had an orifice plate installed on the combined inlet in addition to the insertion turbine flow meters. It is believed that low-loss flow nozzles are installed on SEGS VIII through IX at Harper Lake, California.

The previous flow meters appeared to have three main problems: reliability, availability, and maintainability. Since the initial startup of the plants, there has been concern about the reliability or accuracy of the HTF flow meters. Given the redundancy built into the plants, it is fairly easy to do a simple mass balance to check if the flow entering the solar field is the same as the exiting flow. However, when checks were made, it was common to see a $2 \%$ to $10 \%$ discrepancy between inlet and outlet mass flows. The previous HTF flow meters experienced poor availability since initial startup. On a number of occasions, only one or two of the flow meters were 
operational at a given plant. Finally, the flow meters required a significant amount of attention to keep them operational because of fouling from the HTF, mechanical damage to the flow elements, and transmitter failure or calibration drift.

\section{Scope}

This subtask reviewed the flow meters then used at the SEGS projects, evaluated the problems with each type to determine which flow meter or combination of flow meters provided the best results, and investigated other vendors and types of flow meters (volumetric or mass) that might yield better results.

The following list gives the desired flow meter characteristics:

1. Accuracy of $\pm 0.5 \%$ of flow rate.

2. Rangeability of 16:1 minimum.

3. Operate to $750^{\circ} \mathrm{F}$.

4. Flow meter's performance should be insensitive to the changes in physical properties of the HTF, such as density and viscosity.

5. The flow meter must be maintainable without requiring draining the HTF from the system.

6. Reliability in cold weather.

7. Simple retrofit to existing flow meters.

\section{Flow Meter Test}

A flow meter test installation was set up at SEGS IV in 1993. Flow meters tested included the existing insertion turbine and annubar-type flow meters, in-line vortex flow meter, insertion vortex flow meter (rebuilt from an existing turbine flow meter), a new sonic flow meter under development by a manufacturer, and a transit-time sonic flow meter. The flow meters were tested in series on a single header. A data recorder was set up to record the flow from all of the flow meters simultaneously.

\section{Results and Observations}

Comparative flow meter testing in the inlet to the east field of SEGS IV resulted in a better understanding of each type of flow meter.

Whereas earlier testing in this task supported adoption of the insertion vortex, later experience showed that the insertion turbine meters had several modest advantages with respect to cost, turndown ratio on the flow, and temperature capability (EMCO ${ }^{*}$ builds both types). Turndown ratio is important at the Kramer Junction SEGS plants because HTF is circulated 24 hours a day and flow indication must be shown. The vortex meters often cut out at these low flow rates, which was undesirable for the operator. Reliability and maintenance requirements appear to be

\footnotetext{
"EMCO, 600 Diagonal Hwy., Longmont, Colorado 80501, (303) 651-0550.
} 
good for each. Both types are acceptable - the final choice depends on the parameters of the particular application. Insertion meters are recommended in this application because they can be repaired while the process is on line.

In our view, flow nozzles and annubars suffer in measurement accuracy, and the latter are subject to plugging if the working fluid carries particulates or is subject to plating out (which can sometimes be a problem with the solar field HTF). Because these last two meters measure pressure differences across the meter, turndown ratio and accuracy are dependent on very good signal transmitters, which may have to be stacked to get a high turndown.

Table R-1 summarizes these observations on flow meter applicability to the Kramer Junction SEGS plants. The measurement accuracy given in the table is for the meter, and appropriate signal transmitters are required to achieve this accuracy in the final readout of the data acquisition system.

While the choice of the exact models and manufacturers of flow meters selected for another application is dependent on the exact parameters of that application as well as on commercial terms, we can state that the meters finally selected at the Kramer Junction SEGS plants were supplied by Panametrics ${ }^{\dagger}$ (sonic meter); from a maintainability point of view, the acoustic meter was highly desirable because it was easily attached to the outside of the pipe and was readily accessible. During the Operation and Maintenance Improvement Program, KJC Operating Company (KJCOC) continued to work with Panametrics Inc. to improve the accuracy of the meter. After a few years of trial and error, an acceptable acoustical meter was finally developed. KJCOC subsequently replaced all prime HTF flow meters at the five Kramer Junction plants with the new acoustical flow meters.

Table R-1. Summary of Flow Meter Attributes for SEGS Applications

\begin{tabular}{|c|c|c|c|c|c|c|}
\hline Type & $\begin{array}{l}\text { Min } \\
\text { Flow }\end{array}$ & $\begin{array}{c}\text { Typical } \\
\text { Accuracy } \\
( \pm \%)\end{array}$ & $\begin{array}{l}\text { Maintain- } \\
\text { ability }\end{array}$ & $\begin{array}{c}\text { Reli- } \\
\text { ability }\end{array}$ & Cost & Notes \\
\hline $\begin{array}{l}\text { Vortex } \\
\text { insertion } \\
\text { inline }\end{array}$ & $\begin{array}{l}\text { mid } \\
\text { mid }\end{array}$ & $\begin{array}{c}1 \\
0.7\end{array}$ & $\begin{array}{l}\text { good } \\
\text { good }\end{array}$ & $\begin{array}{l}\text { good } \\
\text { good }\end{array}$ & $\begin{array}{l}\text { low } \\
\text { high }\end{array}$ & $\begin{array}{c}\text { see (a) } \\
\text { see (a) and (b) }\end{array}$ \\
\hline Sonic & low-mid & 1 & very good & good & mid & $\begin{array}{l}\text { ultimately } \\
\text { selected }\end{array}$ \\
\hline $\begin{array}{l}\text { Turbine } \\
\text { insertion } \\
\text { inline } \\
\end{array}$ & $\begin{array}{l}\text { low } \\
\text { low }\end{array}$ & $\begin{array}{c}1 \\
0.5 \\
\end{array}$ & $\begin{array}{c}\text { good } \\
\text { ok }\end{array}$ & $\begin{array}{c}\text { good } \\
\text { ok }\end{array}$ & $\begin{array}{l}\text { low } \\
\text { mid }\end{array}$ & $\begin{array}{l}\text { see (a) } \\
\text { see (b) }\end{array}$ \\
\hline $\begin{array}{l}\text { Flow } \\
\text { Nozzles }\end{array}$ & mid & $1-2$ & good & good & mid & $\operatorname{see}(c)$ \\
\hline Annubar & high & $1-2$ & ok & ok & low & see (c) \\
\hline
\end{tabular}

(a) Can repair while the process is on-line via a hot tap valve or removable sensor.

(b) Repair requires removing meter from the line by diverting flow through bypass line.

(c) Good transmitters, sometimes stacked, are very important to turndown and accuracy.

\footnotetext{
${ }^{\dagger}$ Panametrics, 221 Crescent St., Waltham, Massachusetts 02154, (617) 899-2746.
} 
This page intentionally left blank. 


\section{Appendix S \\ WeATHER PREDiction to SUPPORT OPERATION AND MAINTENANCE PLANNING}

\section{Background}

Accurate foreknowledge of the weather would be of great value to both operation and maintenance (O\&M). Typically, KJC Operating Company (KJCOC) has relied on "out-the-window" observations and normal weather forecasts to make weather decisions. With respect to operations, the near-term gas-use strategy would be better defined, allowing operational decisions on boiler operation during the next several days (Should it be fired, or not? If so, when?). Good decisions will increase the effectiveness of gas use, thus increasing total electrical output. With respect to maintenance, a few days' warning on weather can permit the planning of minishutdowns during a weekend or weekdays when poor weather would otherwise limit solar production, or it can prevent taking a plant off-line for maintenance when solar conditions are good.

\section{Direct-Normal Insolation Forecasting Trial}

This trial was carried out to evaluate a quantitative direct-normal insolation (DNI) forecasting methodology in development by Dr. Todd A. Cerni under contract to the National Renewable Energy Laboratory. The prediction of DNI had not been attempted before, and it is difficult mainly because of the large day-to-day variations. The trial was conducted during May and June 1996.

Observations of local sky conditions can typically provide only a 0 - to 1-hour solar forecast because of visibility limitations alone. Furthermore, comparison of hourly surface reports from nearby Edwards Air Force Base against satellite data demonstrate that local cloud observations often suffer from systematic errors. Cerni concluded that climatology and persistence were unreliable forecasting tools because they are fundamentally incapable of predicting large day-today variations in solar DNI. (This conclusion is not necessarily shared by KJCOC.) It is precisely these large day-to-day variations in measured daily total DNI that are of the most interest for maintenance planning.

Cerni accesses real-time atmospheric data directly from the University of Wisconsin Space Science and Engineering Center (UW SSEC), via a full-time, dedicated Internet link. He then analyzes that data using the McIDAS (Man-computer Interactive Data Access System) tool set. The McIDAS system represents a $20+$ year development effort by the UW SSEC and the McIDAS Users Group, which consists of a large international assembly of universities, corporations, and national laboratories. The types of data used in the solar DNI forecasting include (a) visible and infrared GOES-9 (satellite) imagery at both full and reduced resolution, (b) National Weather Service hourly surface observations, (c) National Weather Service upper air of radiosonde observations (RAOB), and (d) National Oceanographic and Atmospheric Administration numerical weather prediction model output. Cerni developed a ten-step, quantitative DNI solar forecasting methodology for daily generation of 12- and 36-hour 
forecasts. The solar forecasting methodology includes Cerni's numerical models plus a heavy reliance on the McIDAS analysis tools, in the man-computer interactive mode.

The purpose of the KJCOC solar forecasting trial was to (a) provide practical DNI solar forecasting experience in an operational mode, (b) allow the KJCOC staff to become familiar with the daily forecasting products, and (c) provide a basis for comparison between the satellite forecasts and those derived from climatology or persistence (staff observations). Day 1 and Day 2 DNI solar forecasts, nominally representing 12- and 36-hour forecasts, were sent each morning to $\mathrm{KJCOC}$ via fax transmission. Detailed analysis focused on the last three weeks of the forecasting trial, treating the first two weeks as a training period. This trial period was short because of lack of funds, and occurred during a summer weather period when daily changes were modest compared to, say, the winter months. Each forecasting product consisted of a single page of combined text and graphics, including a graph of the DNI (watts per square meter) versus time curve, plus the hourly and daily totals of DNI. The daily total DNI (kilowatts per square meter per day) is the primary quantity of interest for improved maintenance planning.

The average absolute value error of the new forecasts was $0.33 \mathrm{kWh} / \mathrm{m}^{2} / \mathrm{day}$, which equaled about $26 \%$ of that produced by more traditional methods. The average absolute value error of the new Day 2 forecasts was $0.87 \mathrm{kWh} / \mathrm{m}^{2} /$ day, which equaled $47 \%$ of that produced by the other methods.

\section{Commercial Weather Prediction Services}

The above method showed potential, though a longer trial would be necessary to demonstrate its full and true value to O\&M activities at Kramer Junction, California. Moreover, the projected cost for such a service, while a preliminary estimate, indicated that the annual cost might approach $\$ 100,000$.

$\mathrm{KJCOC}$ has also been exploring less expensive commercially available weather forecasting services as part of the O\&M budget (i.e., not a Sandia National Laboratories/KJCOC project). These services cannot predict DNI levels, but instead provide area-specific weather forecasts that can aid in maintenance and gas-use strategy planning.

The current system - the DTN Weather Center-accesses weather data via a commercial satellite broadcast. Area-specific information can be viewed by KJCOC staff at a user station in the Engineering trailer, including satellite maps, current weather conditions, short- and long-range forecasts, daily forecasts, and prior weather data. This service costs less than $\$ 1,000$ per year.

An example of another system under consideration is a customized service called AccuWeather. This company, with a large staff of professional meteorologists, will provide five-day weather forecasts for the Boron, California, area, which include sky cover and precipitation during the daylight hours. Weather data resources similar to the DTN package are also included, along with consultation rights with their meteorologists. The cost of this service would be approximately $\$ 3,500$ per year. Access to KJCOC staff would be site-wide via Internet connection on the site network. 


\section{Appendix T \\ HIGH-WIND OPERATING STRATEgY}

\section{Background}

Solar field operation becomes quite challenging during times of high wind velocities at the Kramer Junction, California, site. Mirrors that are broken by wind forces may subsequently initiate a chain reaction, breaking glass envelopes of the heat collection elements (HCEs) and/or more mirrors. Consequently, collectors are stowed when wind speeds reach 35 miles per hour, or selected solar collector assemblies (SCAs) are stowed to provide wind protection to the rest of the solar field. In the stow mode, the collectors are designed to withstand winds of 80 miles per hour. Longer operation hours without wind protection may be possible if the transient wind loading could be measured as a function of tracking angle.

Earlier attempts were made to characterize typical wind loads on parabolic trough collectors in wind tunnel tests in 1980 at Colorado State University. The purpose of the tests was to determine forces on the collectors at different tracking angles. Next, another field test was performed at the solar electric generating system (SEGS) plants in 1992 to observe the wind-flow pattern, qualitatively rendering some initial information about vortices in the interior part of the solar field. These observations have continued through the present and are the basis for the current wind protection strategy now in use.

Because of the lack of information about wind speeds around the collectors, solar field operation during the gusty wind conditions is presently very conservative to protect the solar field from damage. This task was initiated to gather data to support a revised wind-protection strategy to maximize the solar field availability and minimize mirror and HCE breakage while maintaining maximum power output.

\section{Scope of Work}

A series of experiments were conducted to test this hypothesis by evaluating ten wind-protection modes spanning the entire normal operating range while the solar fields were subjected to gusts of 25 to 38 miles per hour. A wind-protection mode is a solar field configuration method where selective SCAs are strategically positioned in order to diffuse the energy content of the wind. Analysis has resulted in comprehensive criteria governing wind protection of the solar field based on prevailing factors, such as peak wind velocity, wind frequency, and collector angle. Furthermore, optimum wind-protection modes have been chosen to achieve minimal solar field damage while maximizing long-term solar thermal output.

This test was performed at Kramer Junction in the SEGS V solar field. All test instrumentation was located either on or near SCA $22 \mathrm{O}$ as shown in Figure T-1. This location is near the center of the Kramer Junction site.

A wind tower placed midway between SCAs $22 \mathrm{O}$ and $23 \mathrm{O}$ had unidirectional anemometers at 3,5 , and 10 meters off the ground. Pressure probes (Kiel probes) were mounted at SCA $22 \mathrm{O}$ on both sides of the 1, 2, 3, and 4 mirrors as shown in Figure T-2. 


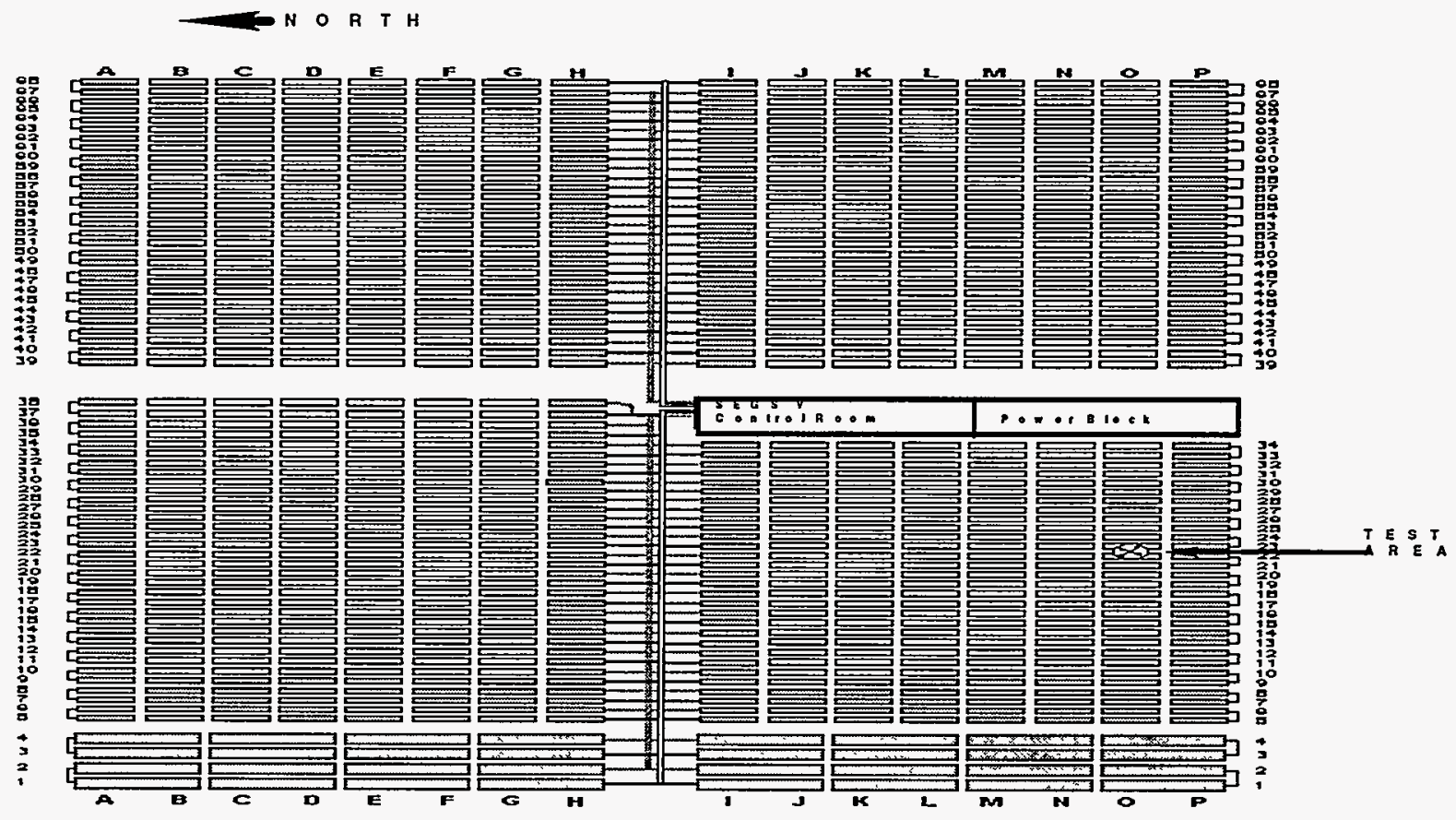

Figure T-1. Location of the test.

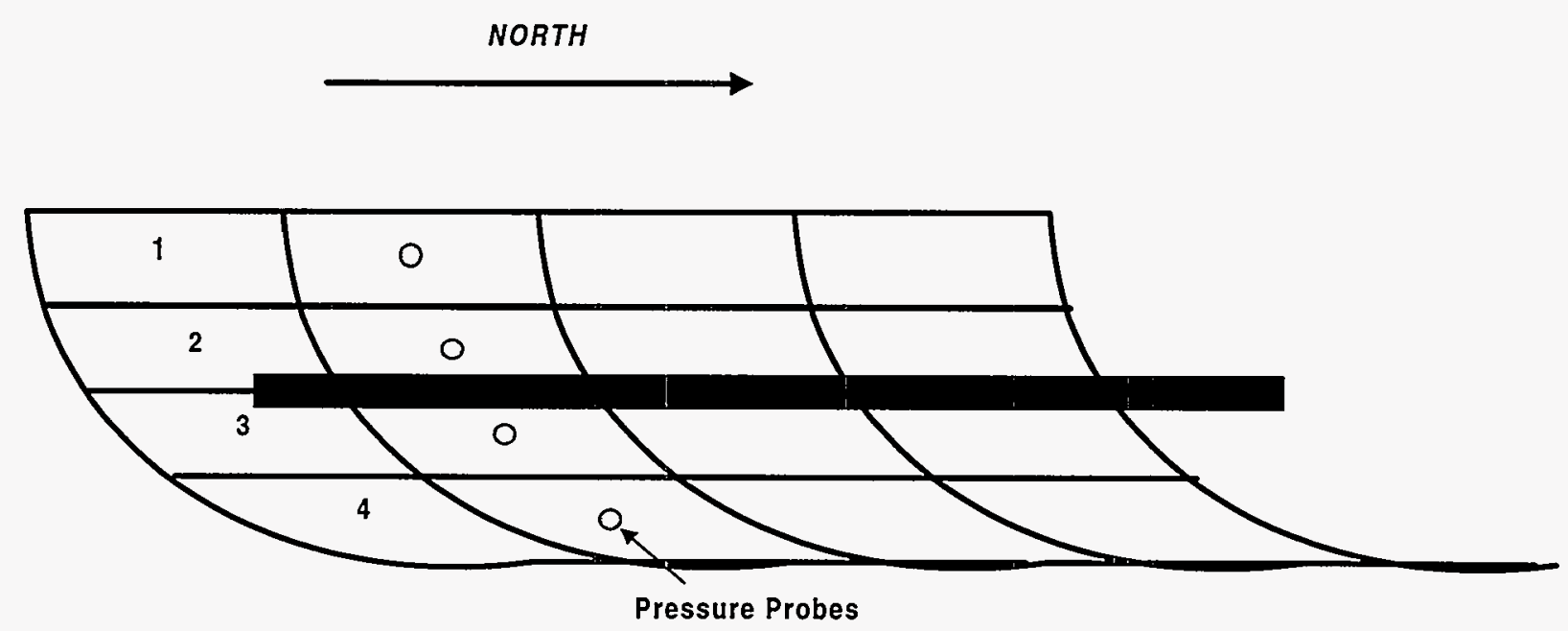

Figure T-2. Location of pressure probes.

To calculate the impact energy imparted to a mirror segment by wind, the shortest duration of data acquisition interval would render the most accurate results on wind dynamics. In this testing, sample data were scanned and stored at one-second intervals. The datalogger was attached to a remote computer via a null modem that allowed data to be instantaneously stored on a mass storage system.

A factor to be considered when conducting the tests was that wind speeds should be as constant as possible to avoid the pitfalls of extrapolation. Because it was unlikely to expect a constant 
wind speed for every test, a target wind speed of 30 miles per hour (all wind speeds mentioned hereafter refer to the wind speed measured at the wind tower, 10 meters off the ground) was arbitrarily chosen as a reference. Because it was mandatory that all testing be conducted in actual gusty conditions, a major condition to be met was to wait for the winds to increase beyond 25 miles per hour. Every effort was made to conduct testing at night to minimize the impact on the SEGS solar electrical production. Testing was done between March and May 1996, because these months are typically the windiest at Kramer Junction.

While keeping the tracking position of the field constant, different wind protection strategies were employed for a period of time during a gusty wind period. Net impact pressure on the mirrors was measured and compared with the condition when no wind protection was in effect. A sufficient number of gust events were recorded to ensure that they were statistically significant for generalization.

\section{Results}

For more detail on the analytical approach, see Optimized Solar Field Operation During High Wind Velocities at the Kramer Junction SEGS by Determining Transient Forces, N. Amarnani, R. Cable, and G. Cohen, Solar Energy Forum (American Society of Mechanical Engineers session), Washington, D.C., April 1997

Looking at the magnitude of forces on all four mirrors over the full spectrum of tracking positions, this work concluded that a solar field might benefit from a wind protection strategy past $45^{\circ}$ up to $120^{\circ}$. At positions less than $45^{\circ}$ and past $120^{\circ}$, the solar field has an inherent characteristic of self-protection. Considering the relative potency of the wind-protection modes, mode WP43 (every fourth row and the southernmost channel in wind protection) was shown effective between $60^{\circ}$ and $105^{\circ}$.

However, it was observed that the test SCA was subjected to high-impact pressures at tracking positions of $45^{\circ}$ and $120^{\circ}$ as well. Regretfully, none of the tested wind-protection strategies offered any relief. Until flow patterns are investigated in more detail and other strategies designed, the tracking positions in the proximity of $45^{\circ}$ and $120^{\circ}$ are recognized to be vulnerable to collector damage in gusty wind conditions.

Another concern is the requirement to switch the position of protective collectors from $10^{\circ}$ in the morning to $170^{\circ}$ past solar noon. Presumably, when gusty weather persists in the morning, a wind-protection mode has been invoked around $60^{\circ}$. As solar noon approaches, the field configuration is being subjected to higher loads. The effect on SCAs as they pass through $90^{\circ}$ has not been specifically studied; however, in a static mode, this is one of the most vulnerable positions. Further investigation may be appropriate to observe this transitory action. Our present recommendation is to switch the wind-protection mode in some kind of incremental fashion, in contrast to positioning all the guarding SCAs simultaneously, to reduce the risk of catastrophic damage to the solar field in case a sufficiently powerful gust coincides with this transitory mode.

Two other issues deserve comment. The first issue is to define the wind conditions that require putting the solar field in a wind-protection mode. We have observed that wind protection should be considered when two or more gusts of 25 miles per hour are observed within a 30-minute interval, because it has been observed that gusts pick up following these events. To enter 
wind-protection mode, frequent gusts in the range of 30 to 35 miles per hour should be observed. The second issue is to verify the wind speed at which the solar field must be stowed to ensure protection. This issue was not addressed here because of the lack of information about the loading characteristics on the solar field.

In conclusion, the recommended strategy for wind protection is WP43 (every fourth row in wind protection and the southernmost channel) between tracking angles of $45^{\circ}$ and $120^{\circ}$. However, an optimum high-wind operating strategy at SEGS plants must incorporate at least five variables such as wind speed, its constantly changing direction, its tracking position, the relative location of the SEGS solar fields on the Kramer Junction site, and the relative position of the SCAs within a SEGS. Generalization of this complex problem may only achieve partial success in reaching the goal of optimized operations at this facility. 


\section{Appendix U SOLAR FIELD FLOW CONTROL}

\section{Background}

To deliver steam at appropriate conditions to the turbine in a solar Rankine cycle, the solar field is designed to deliver heat transfer fluid (HTF) at a specified temperature to the steam generators under variable radiation conditions. In general terms, the total HTF flow rate passing through the solar field is adjusted to achieve the desired outlet temperature. However, the solar field is made up of a number of independent loops feeding into the outlet header. Ideally, each loop will deliver the same HTF temperature to the header to achieve a uniform field outlet temperature. However, in practice, this is often not the case because the outlet temperature of each loop in the solar field is a function not only of the solar radiation, but also of the collector efficiencies, collector status, and the HTF flow rate within that loop. These conditions will vary between loops in a large solar field.

There are several control options available. For a specified level of solar radiation and a given equipment status in an individual loop (e.g., number of operational solar collector assemblies [SCAs], mirror reflectivities, broken mirrors, and damaged heat collection elements [HCEs]), the outlet temperature can be raised or lowered by adjusting the HTF flow rate, or can be lowered by defocusing SCAs. In the Kramer Junction, California, plants, a flow control valve on each loop sets the flow in each loop under varying header conditions. Even in a solar field with identical collectors, flow maldistribution between loops at varying flow rates can occur because of the settings of these valves. Flow distribution can be readjusted by manually changing the setting of loop flow control valves. (At the Solar Electric Generating System [SEGS] IX plant at Harper Lake, California, fixed orifice plates, not valves, determine the loop flow rates.)

Another viable alternative, also considered in the design phase but rejected at that time, is to use automatic flow-control valves (FCVs) to maintain a desired outlet temperature for each loop. This affords much better control of outlet temperature because this method automatically compensates for weather and equipment conditions. However, this approach is also more costly and less reliable from an equipment standpoint. Given its potential advantages, however, a test was undertaken to examine the feasibility and performance of automatic flow control in a prototype test area.

\section{Test Description}

The test area to evaluate automatic loop flow control was set up in SEGS V and is operational. Two loops with automatic control are being compared to two adjacent loops with manual flow valves.

Preliminary tests show smoother flow transitions in transient conditions (e.g., morning startup) because of the new control system. This characteristic will act to reduce HCE tube breakage (which can fail because of asymmetric thermal stresses when the startup flow is too low) and may result in sufficiently higher performance to offset the higher initial costs and ongoing maintenance costs. The test will be of particular interest in the fall and winter when variable weather conditions and lower sun angles will show greater differences than during the summer 
months when the FCVs are typically wide open. During high-wind conditions, the flow in stowed loops can automatically be cut back to reduce heat losses.

The test loops were designated as rows 29/30 A-H (upper field) and I-P (lower field). The test loops consist of one set of upper and lower field loops. (Because of the design of the solar field, the upper and lower fields of loop 29/30 share a common three-inch supply and return pipe. With existing globe valves set identical, the lower field loop receives more flow than the upper because of head pressure.) The reference loops were designated as rows $27 / 28 \mathrm{~A}-\mathrm{H}$ (upper field) and I-P (lower field). The test loops are outfitted with additional thermocouple-type temperature elements-each with a transmitter-located at the inlet and outlet of the loops. A vortex flow meter is installed at the inlet of the loop along with an electrically actuated, hydraulically operated flow control valve (EHFCV). The reference loops are outfitted with identical instrumentation.

These loops, both test and reference, were selected for their similarities in equipment status (e.g., number of operational SCAs, ball joints, broken mirrors, and damaged HCEs) and relative proximity to the power block and thus to the control system, the Westinghouse Distributed Control System (DCS) located in the control room. The DCS was selected for this project for its versatility in programming and control capabilities. The system was set up with a $3^{\circ} \mathrm{F}$ temperature deviation from the desired output set point for the controller to initiate an output signal to the FCVs. By using all the available information (loop flow and outlet temperature, temperature rise, and direct radiation) the DCS will automatically control valve position, which in turn increases or decreases flow using the FCV (EHFCV) to optimize loop outlet temperature during various conditions.

Minimum output signals were established to provide for safety factors in all control ranges. Minimum output signals include the following: FCVs are set at $5 \%$ valve position, radiation is set at less than $350 \mathrm{~W} / \mathrm{m}^{2}$, and ambient temperature is set at less than $50^{\circ} \mathrm{F}$. Any values recognized as below these standards will automatically revert the system to manual, and a $100 \%$ output signal will be sent to the EHFCVs. This control scheme in no way overrides or inhibits field supervisory controller controlled loop protection. Historical trends in the DCS were set up to monitor and graph the inlet and outlet HTF temperatures, HTF flow, valve positions, and radiation.

The DCS logic shown in Figure U-1 has been developed and implemented for automatic temperature control of 29/30 A-H and 29/30 I-P loops (see also Figure U-2). The minimum valve position for FCV-291 (29/30 I-P loop) and FCV-29H (29/30 A-H loop) was set at 5\%. This valve position allows adequate flow per loop with $\mathrm{P} 100 \mathrm{~A} \& \mathrm{~B}$ HTF pumps in service and on minimum speed during plant startup. A $3^{\circ} \mathrm{F}$ temperature deviation from the desired set point has been established for the controller to initiate an output signal to the FCVs. The FCV operates proportionally to the temperature increase or decrease in the loop. The minimum output signal from the controller to the FCV is set at $5 \%$. If solar radiation falls below $350 \mathrm{~W} / \mathrm{m}^{2}$ and the ambient temperature falls below $40^{\circ} \mathrm{F}$, the FCV will revert to manual and open to $100 \%$. The DCS logic has been set up to select the highest value from the two pyrohilometers in the event that one of the instruments fails. 


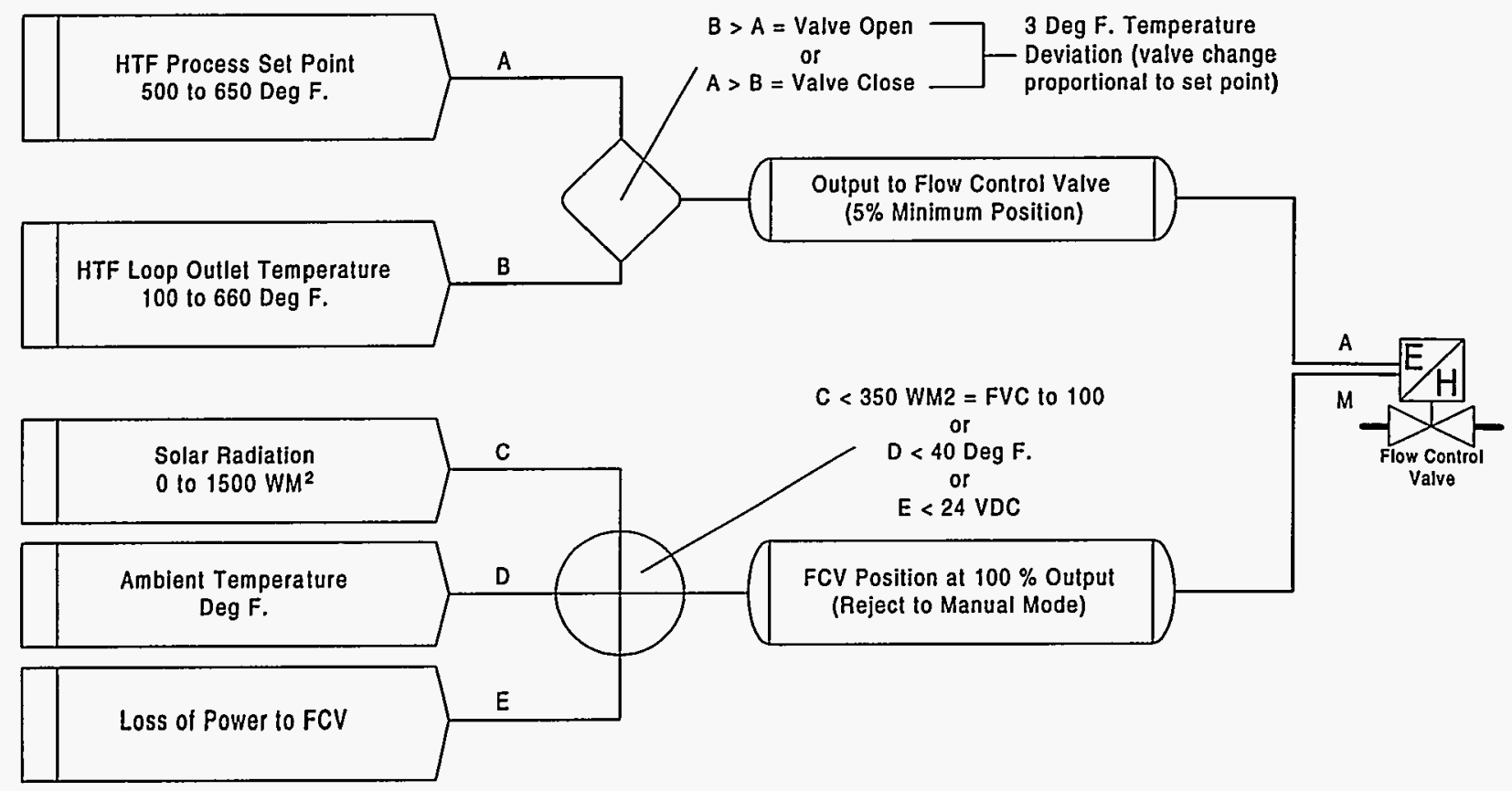

Figure U-1. DCS logic for automatic temperature control.

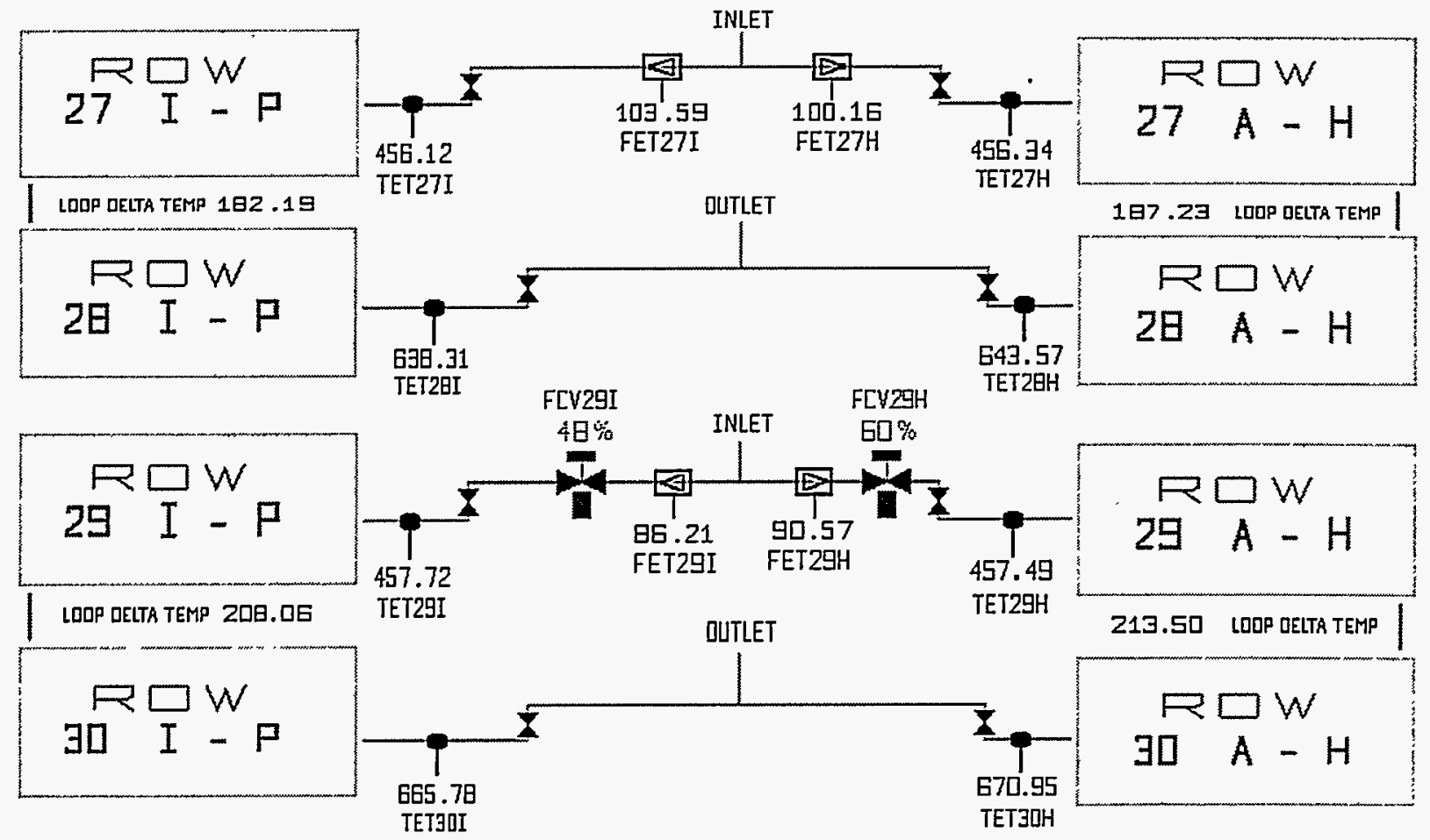

Figure U-2. DCS operator screen depicting SEGS V loop automation. 


\section{Test Results}

Initial testing was performed during a typical unit startup in August 1997. Both loops were placed in automatic control with a set point of $600^{\circ} \mathrm{F}$ on the controllers. As the radiation and outlet temperature increased, the FCV positions increased. This allowed the appropriate increase in flows according to the logic, which resulted in a more controlled startup. Desired set point temperatures were obtained at the outlets of each loop (test) with no overtemps on the affected SCAs. During this test, the control loops reached the desired outlet temperature before all other loops in the field. Because of FCV positioning problems, testing was postponed until late November.

Testing proceeded early in November and focused on a more closely monitored startup and steady state operation. Differences were noted between the upper and lower field loops and their corresponding reference loops. As shown in the graph labeled "Nov 05, 1997" in Figure U-3, the initial startup temperature increase in the test loops was substantially quicker than the reference loops, without damage to the equipment. As the radiation ${ }^{*}$ increased with a subsequent rise in outlet temperature, the EHFCV opened to compensate by increasing the HTF flow. Note that the flow in the reference loops was initially higher because it is determined by the flow needs of the entire field, not just the needs of a single loop. Had the entire field been outfitted with the EHFCV, the startup time would have been decreased substantially. This was demonstrated throughout all of the startup tests.

Loop outlet temperature was also shown to be more stable using the control scheme during steady state operations. As shown in the graph labeled "Nov 14, 1997" in Figure U-3, the outlet temperature is steadier in the test loops, and the reaction time to a decrease in direct radiation was notably smoother, thus maintaining a higher outlet temperature through the transition period. This smooth transition equates to a reduction in the loss of load the unit would experience with a more delayed reaction (operator quickness) and abrupt manual adjustments. Thus, it appears clear that loop automation is particularly suitable for winter operations where, during steady state operations, the system will act in the "flow-balance" mode. In summer, outlet temperatures are considerably higher and steady state operations normally equate to wide-open valves and pumps at full throttle. However, during summer, automatic flow control should still provide benefits at startup, achieving a faster startup within the bounds of equipment safety parameters.

\section{Recommended Near-Term Use of Loop Flow Control}

As emphasized in the main report, another benefit of loop flow control is the ability to reduce flows in loops isolated during periods of high winds. During these periods, with winds 35 miles per hour and higher, a portion of the field is positioned (out of focus) to protect the remainder of the field. Now, these positioned loops are left with the globe valves open, thus quenching the return oil headed to the power block. (During this time, personnel are restricted from the fields for safety reasons and cannot perform the valve isolations.) With flow automation, these loops could be partially isolated, thus reducing the amount of cold oil entering the hot return header.

\footnotetext{
* The diagram shows direct-normal insolation (DNI), whereas the loop heat input and temperature increase are actually responding to the flux in the plane of the collector. In winter, the DNI peaks about noon but the planar flux peaks at about 10 a.m. and 2 p.m., decreasing toward noon and then rising again. These effects influence the outlet temperature and flow results shown in the diagram.
} 
Outlet Temperature \& Flow Comparison

Controlled \& Non-Controlled Loops

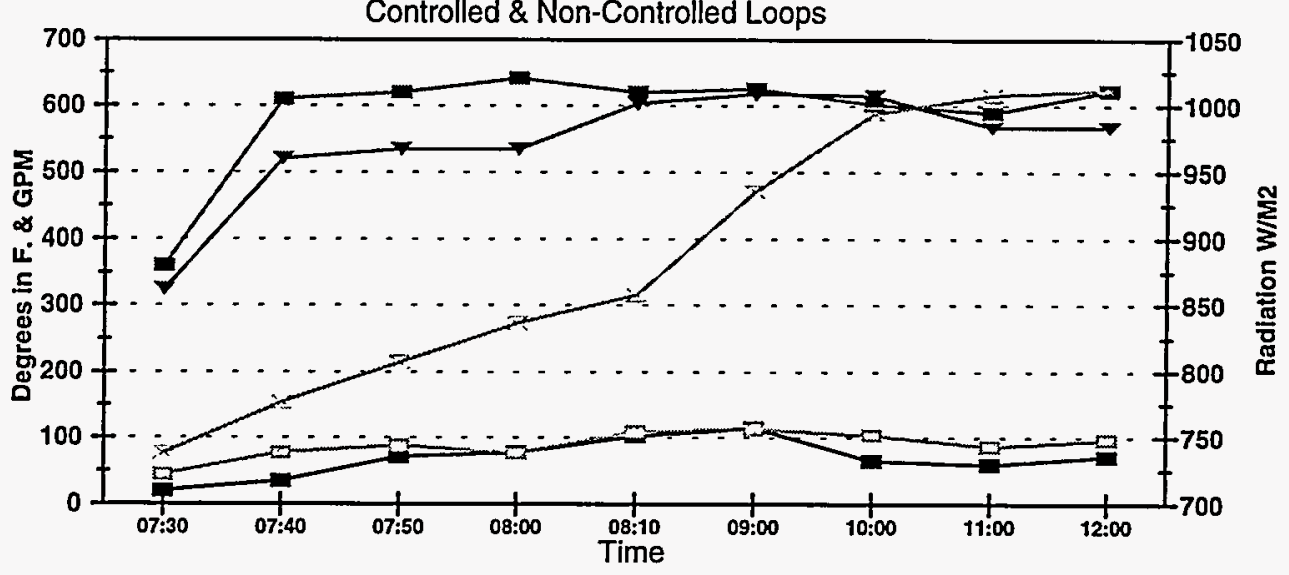

- Non-Controlled Loop Flow

$\rightarrow-$ Controlled Loop Outlet Temp $\rightarrow \longrightarrow$ Non-Controlled Loop Outlet Temp

Nov 05, 1997

$-2-$ Radiation

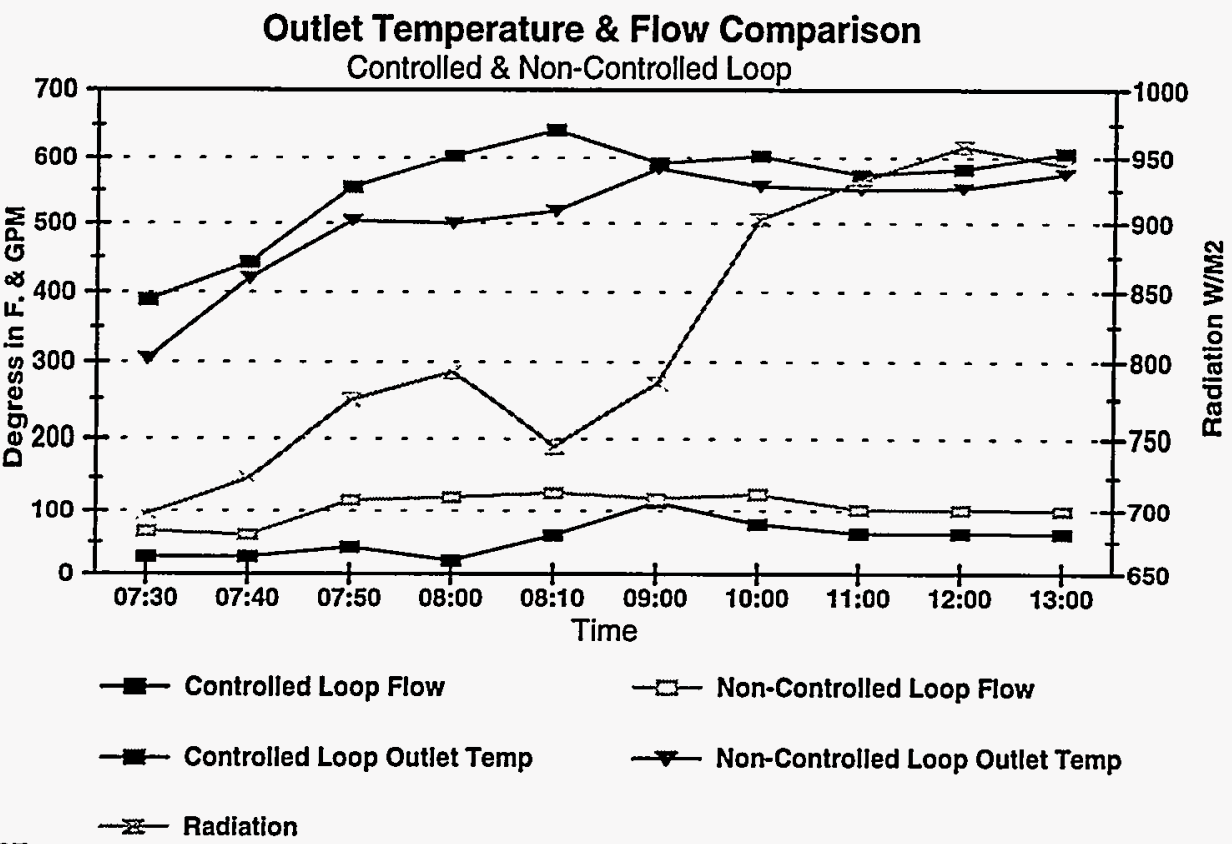

Nov 14, 1997

Figure U-3. Results for November 5 and 14, 1997. 
This page intentionally left blank. 


\section{Appendix V ANALYTICAL MODEL OF SOLAR COLLECTOR FIELD*}

\section{LS-2 Performance Equation}

The solar field thermal performance model is based on tests conducted by Sandia National Laboratories (SNL) for an LS-2 collector on the rotating platform (see Appendix A). From the results of these tests, efficiency equations were derived for heat collection elements (HCEs) with vacuum, air in the annulus, or for bare tubes as a function of fluid temperature, incident angle, insolation, and, for bare tubes, wind speed.

In defining the thermal efficiency, $\eta_{\text {th }}$, of the collector as the ratio of absorbed power (in percent), $Q_{a b s}$, to the direct-normal insolation, I (in watts per square meter), the general equation

$$
\eta_{\mathrm{th}}=\frac{Q_{a b s}}{I}=K[A+B(\Delta T)]+C \frac{\Delta T}{I}+\mathrm{D} \frac{\Delta T^{2}}{I}
$$

was found adequate for the description of all HCE conditions except for bare tubes. In this equation, A accounts for the optical efficiency of the trough and the absorptivity of the selective coating without considering the losses at the end of a collector row. The coefficients $B, C$, and D describe the heat losses of the HCE dependent on its conditions with $\Delta \mathrm{T}$ as the temperature difference between the heat transfer fluid (HTF) and the ambient in degrees Kelvin. The incident modifier, $\mathrm{K}$, is a function of the incident angle Ia:

$$
K=\cos (I a)-0.0003512(I a)-0.00003137(I a)^{2} .
$$

For bare tubes, no dependency on insolation was found but there was a strong influence by the wind. The following equation is given for cermet as the selective coating:

$$
\eta_{\text {bare }}=74.7-0.042(\Delta T)-0.000731(\Delta T)^{2}-0.00927(\Delta T) \nu_{\text {wind }}
$$

which can be transformed to a form similar to equation (1):

$$
\eta_{\text {bare }}=K[\underbrace{74.7}_{A_{\text {bare }}}-\underbrace{\left(0.042+0.00927 \frac{v_{\text {wind }}}{K}\right)}_{B_{\text {bare }}}(\Delta T) \underbrace{+0}_{C_{\text {bare }}}(\Delta T) \underbrace{-0.000731}_{D_{\text {bare }}}(\Delta T)^{2} .
$$

Table V-1 summarizes the parameters as they were found in the test results.

\footnotetext{
- This material was taken from F. Lippke, Simulation of the Part-Load Behavior of a 30 MW SEGS Plant, SAND951293, Sandia National Laboratories, Albuquerque, New Mexico, June 1995.
} 
Table V-1. LS-2 Thermal Performance Coefficients

\begin{tabular}{|l|c|c|c|c|}
\hline & A & B & C & D \\
\hline Cermet, vacuum & 73.3 & -0.007276 & -0.496 & -0.0691 \\
\hline Cermet, air & 73.4 & -0.004683 & -14.40 & -0.0637 \\
\hline Cermet, bare & 74.7 & $-0.042-0.00927^{*}$ V $_{\text {Wind }} / \mathrm{K}$ & 0.00 & $-0.000731^{*} \mathrm{I}$ \\
\hline $\begin{array}{l}\text { Black Chrome, } \\
\text { vacuum }\end{array}$ & 73.6 & -0.004206 & 7.44 & -0.0958 \\
\hline Black Chrome, air & 73.8 & -0.006460 & -12.16 & -0.0641 \\
\hline
\end{tabular}

\section{Solar Collector Assembly End Losses}

The efficiency equations derived by Dudley, et al. (1994) in Appendix A do not include the end losses of a parabolic trough row. These are simply a function of the focal length, $f$, of the collector and the incident angle, Ia, as shown in Figure V-1.

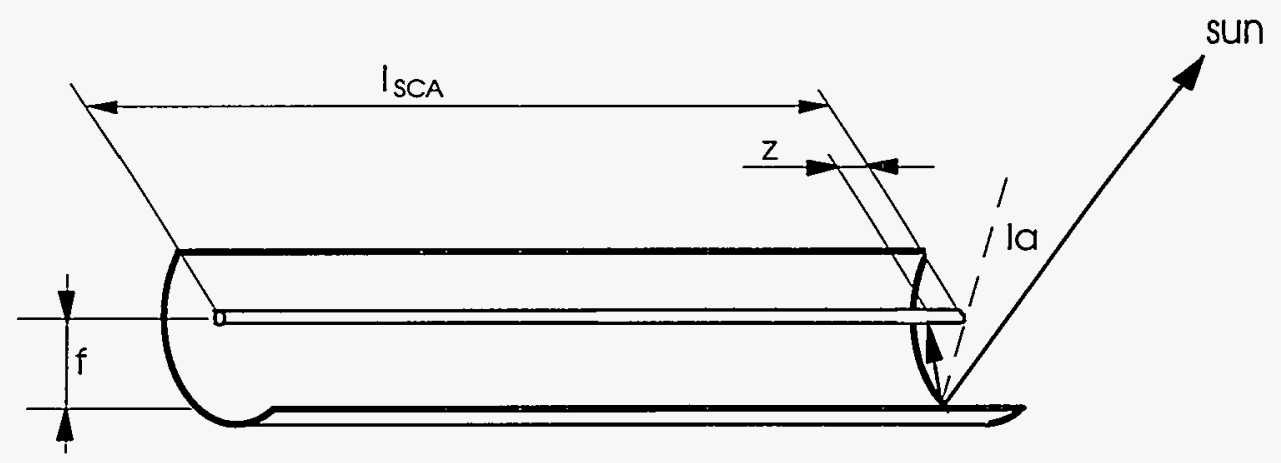

Figure V-1. End losses of a collector row.

The receiver length, $z$, which is not illuminated by the sun, then is

$$
z=f \tan (I a) .
$$

Relative to the total length, $\mathrm{l}_{\mathrm{SCA}}$, of the solar collector assembly (SCA), the amount of heat concentrated on the whole receiver tube therefore has to be reduced by the factor $\mathrm{M}$ :

$$
M=\frac{l_{S C A}-z}{l_{S C A}}=1-\frac{f \tan (I a)}{l_{S C A}}
$$

M has to be included in equation (1) so that this becomes

\footnotetext{
${ }^{\ddagger}$ As an example for an LS-2 collector $\left(f=1.49 \mathrm{~m}, \mathrm{l}_{\mathrm{SCA}}=47.1 \mathrm{~m}\right)$, the factor $\mathrm{M}$ becomes $94.9 \%$ at noon on January 1 $\left(\mathrm{Ia}=58^{\circ}\right)$
} 


$$
\eta_{\mathrm{th}}=K M[A+B(\Delta T)]+C \frac{\Delta T}{I}+\mathrm{D} \frac{\Delta T^{2}}{I}
$$

\section{Performance Equation Considering Different HCE Conditions}

In the calculations carried out in this study, not every HCE in the solar field is treated separately. Only a single element with a performance equation like equation (1) is used. The parameters for this equation, therefore, have to account for the different HCE types found in the field as well as for broken mirrors and, what has not yet been mentioned, "fluorescing" tubes. For the latter HCE type, the coating is defective and partially coats the inner wall of the glass envelope, which then reflects the concentrated light so that no or only a little sunlight reaches the absorber tube. This means that such HCEs, which still cause heat losses, can be approximated by using 0 for factor $\mathrm{A}$ in equation (1). The same is true for HCEs whose mirrors are broken.

Another important factor to be included in the calculations is the cleanliness of the mirrors, $\phi_{\mathrm{M}}$. Measurements show that the reflectivity of the mirrors drops considerably between two washing cycles without rain. The measured data can be used to get $\phi_{M}$ by comparing it to the maximum reflectivity achieved right after a wash, which is about $90.5 \%$ for the LS-2 collector:

$$
\Phi_{M}=\frac{\text { actual reflectivity }}{\text { maximum reflectivity }}
$$

Dirt also reduces the transmittance of the glass envelope, but no information is available on that. A reasonable assumption, however, is that the cleanliness or the reduction in transmissivity of the mirrors and the glass envelopes is about the same. Because light has to pass two times through dirt on the mirrors (reflective side at the back of the glass) and one time through the glass envelope, the cleanliness factor for the glass envelope, $\phi_{\mathrm{E}}$, can be calculated by

$$
\Phi_{E}=\frac{1+\Phi_{M}}{2}
$$

Finally, when setting up the parameters for the performance equation, the influence of each HCE type must be weighted. Considering the percentage $\psi_{i}$ of HCEs of a particular state and the assumption that all types of HCEs are distributed homogeneously over the solar field, this can be done by ${ }^{\S}$

$$
\begin{gathered}
A_{\text {Field }}=\left(A_{\text {Bare }} \psi_{\text {Bare }}+\Phi_{E} \sum_{i, i \neq B a r e} A_{i} \psi_{i}\right)\left(1-\varphi_{\text {Lost Mirrors }}\right) \Phi_{M} \Phi_{A} \\
B_{\text {Field }}=\sum_{i} B_{i} \psi_{i}
\end{gathered}
$$

\footnotetext{
${ }^{8}$ Example: If $88 \%$ of the HCEs are intact, $3.0 \%$ contain air, $7.4 \%$ are bare tubes, $1.6 \%$ have defective coatings, and $0.18 \%$ of mirrors are broken, the performance parameters for HCEs with cermet become: $A=72.1$, $\mathrm{B}=-0.00977-0.000686 \mathrm{v}_{\text {Wind }} / \mathrm{K}, \mathrm{C}=-0.8786, \mathrm{D}=-0.0638-5.4094 \mathrm{e}-6 * \mathrm{I}$.
} 


$$
\begin{aligned}
& C_{\text {Field }}=\sum_{i} C_{i} \psi_{i} \\
& D_{\text {Field }}=\sum_{i} D_{i} \psi_{i}
\end{aligned}
$$

with $\phi_{\mathrm{A}}$ as an additional factor that can be used to vary the overall optical efficiency or modify the overall absorptivity and $\varphi_{\text {Lost Mirors }}$ as the percentage of broken mirrors throughout the solar field. Note, that

$$
\sum_{i} \psi_{i}=\psi_{C, V a c}+\psi_{B C, V a c}+\psi_{C, a i r}+\psi_{B C, a i r}+\psi_{b a r e}+\psi_{f l u o r}=1
$$

applies (with $\mathrm{C}=$ cermet $\mathrm{BC}=$ black chrome).

\section{Piping and Expansion Vessel Heat Losses}

In the operation of a distributed solar power plant, the heat losses in all the piping are important and have to be included in the model. Additionally, the heat losses in the expansion vessel, which has a large surface area, should be included in the calculations.

Both heat losses are considered temperature dependent, where a constant solar field outlet temperature is assumed. The following dependency was implemented in the model

$$
\begin{aligned}
\dot{Q}_{\text {Heat Losses }} & =\dot{Q}_{\text {Piping }}+\dot{Q}_{\text {ExpansionVessel }} \\
& =20 \frac{W}{m^{2}} A_{S F} \frac{\overline{\Delta \vartheta}_{S F}}{343^{\circ} C}+2.57 M W_{t h} \frac{\overline{\Delta \vartheta}_{S F}}{275^{\circ} C} .
\end{aligned}
$$

Here, heat losses of all the piping of $20 \mathrm{~W} / \mathrm{m}^{2}$ aperture area, $\mathrm{A}_{\mathrm{SF}}$, are assumed at full solar power at a mean solar field temperature above ambient of about $\overline{\Delta \vartheta}_{S F}=343^{\circ} \mathrm{C}\left(649^{\circ} \mathrm{F}\right)$. The radiation and mixed convection heat losses of the expansion vessel were estimated to be $2.57 \mathrm{MW}_{\mathrm{t}}$ at $300^{\circ} \mathrm{C}\left(572^{\circ} \mathrm{F}\right)$ field outlet temperature $\left(275^{\circ} \mathrm{C}\right.$ mean field temperature) assuming poor insulation conditions experienced in the plant under consideration in 1994.

\section{Validation of the Model}

In order to validate the model, its results are compared to the real plant behavior. The solar field conditions at Kramer Junction, California, are constantly changing, and the actual reflectivity of the mirrors throughout the field are only approximately known, as are the field locations of HCEs with broken glass envelopes or lost vacuum.

In comparing results to real plant data, it is possible to show how different field conditions affect the results. This study shows how the results compare to the real plant conditions for a summer and a winter day. This allows first the optical performance model and then the heat-loss 
assumptions to be validated, because the latter influence the results much more in the winter than in the summer.

\section{Clear Summer Day}

Figure V-2 shows the insolation conditions and the wind speed measured during a clear summer day. For this prediction (see Table V-2), as much information as possible about the solar field conditions for that day were included (base case $\mathrm{S} 1$ ). The mean reflectivity ${ }^{* *}$ of the solar field is set to $87.1 \%$ and a wind speed of $1 \mathrm{~m} / \mathrm{s}(2.2 \mathrm{mph})$ was chosen, which was valid until about 11 a.m. The second case, $\$ 2$, considers a somewhat reduced absorptivity of all bare tubes because of degradation of the selective coating. S3 shows how an additional reduction in the absorptivity of all receiver tubes influences the result.

Figure V-3 compares temperatures predicted by the model for case S3 with actual plant data. Generally, a very good agreement between the model and the real plant behavior is found.

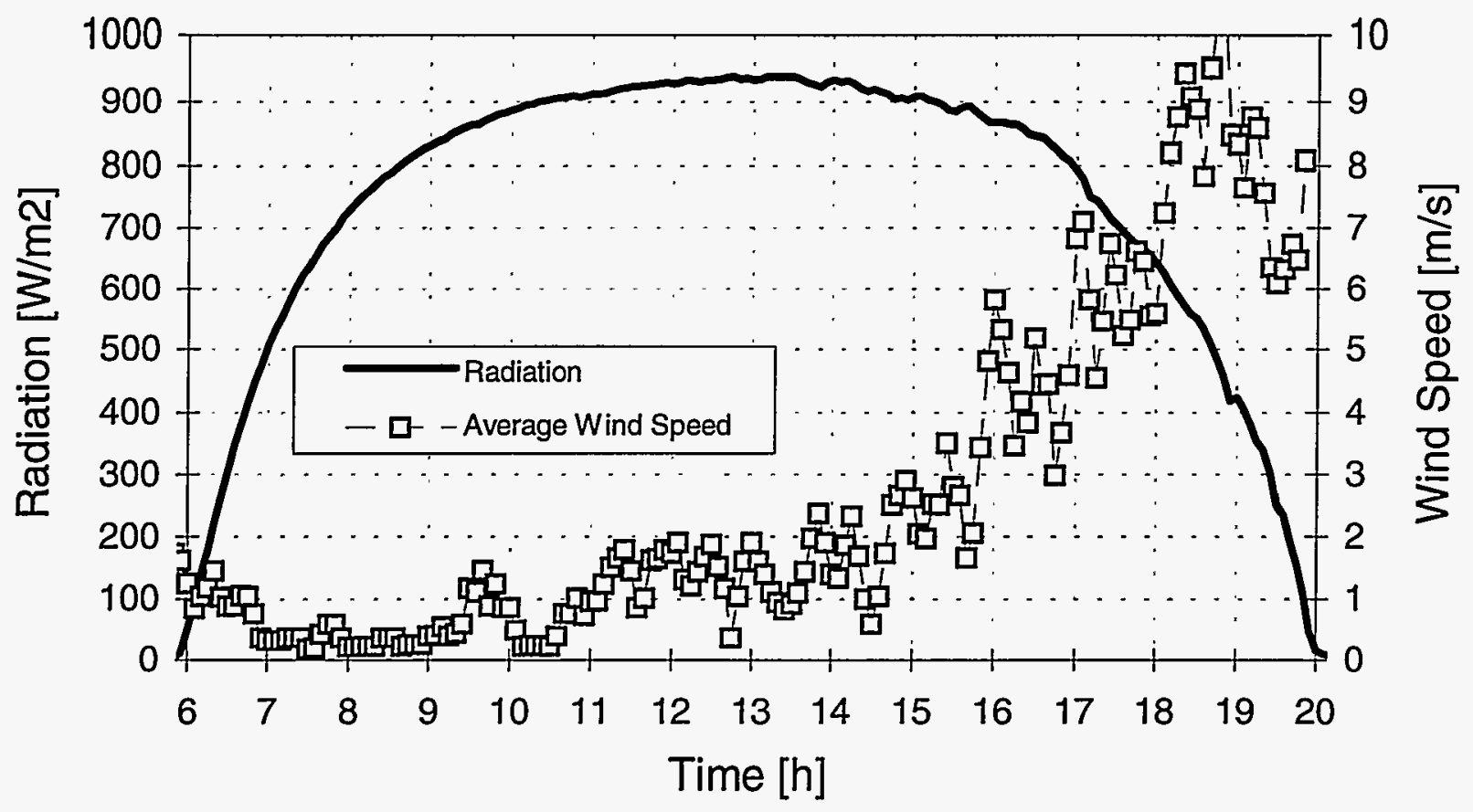

Figure V-2. Insolation and wind speed during a clear summer day.

\footnotetext{
** The optical efficiency has to be scaled by the reflectivity experienced in the solar field compared to the reflectivity assumed for the test collector during the platform tests at SNL. The latter was first assumed to be spray-washed, resulting in a typical cleanliness of $96.3 \%$ or a reflectivity of $90.5 \%$ achieved after a wash. However, after finishing the calculations, it was found that the test collector was hand-washed, resulting in a better cleanliness of the facets. Assuming that the test collector had a reflectivity of about $93 \%$, the calculated gross output therefore has to be multiplied by a factor of $97.3 \%$, resulting in a better comparison to the real, measured output.
} 
Table V-2. Case Definitions for the Summer Day

\begin{tabular}{|l|l|}
\hline \multicolumn{1}{|c|}{ Case } & \multicolumn{1}{c|}{ HCE/Field Conditions } \\
\hline S1 & $88.8 \%$ with vacuum \\
(base case) & $2.8 \%$ with air \\
& $6.2 \%$ bare (wind speed $1 \mathrm{~m} / \mathrm{s}=2.2 \mathrm{mph}$ ) \\
& $1.6 \%$ with defective coating \\
& $0.6 \%$ affected by cooling tower problem (5 SCAs out of service) \\
& $0.27 \%$ of mirrors broken (100\% of SCAs tracking) \\
& Mirror Reflectivity 87.1\% \\
\hline S2 & Case S1 with an additional reduced absorptivity of bare tubes to 75\% because \\
& of degradation \\
\hline S3 & Case S2 with additional "field degradation factor" $95 \%$ \\
\hline
\end{tabular}

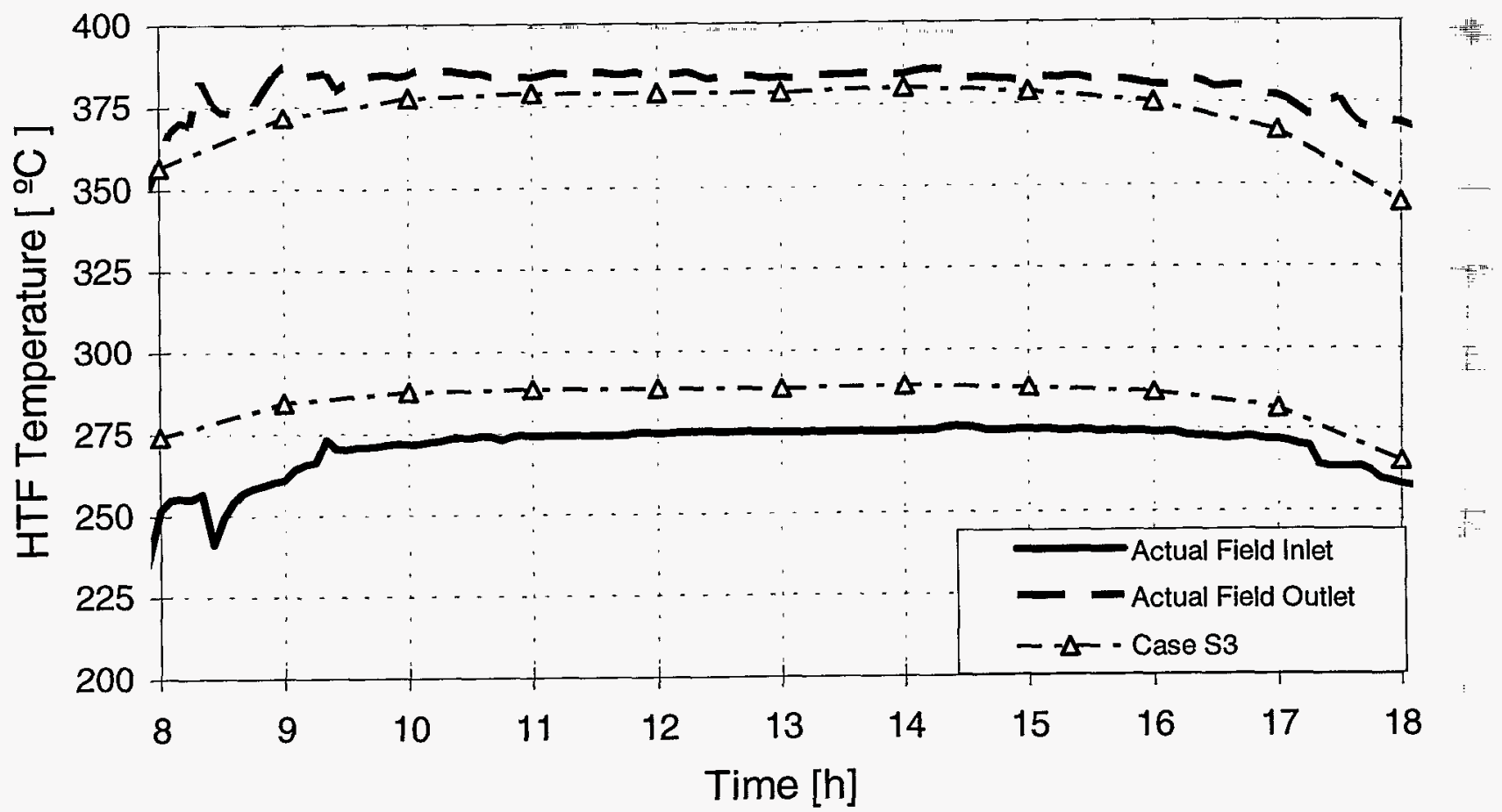

Figure V-3. HTF temperatures during a clear summer day. 


\section{Clear Winter Day}

Figure V-4 shows the insolation conditions and the wind speed for a winter day. Again, calculations were made for this day assuming different solar field conditions, as summarized in Table V-3.

Considering the summer day, a combination of reduced optical efficiency and higher thermal losses might be adequate to represent the real conditions. Comparing the predicted temperatures for case W3 with the measured data again, temperatures are seen to be close to the actual (see Figure V-5).

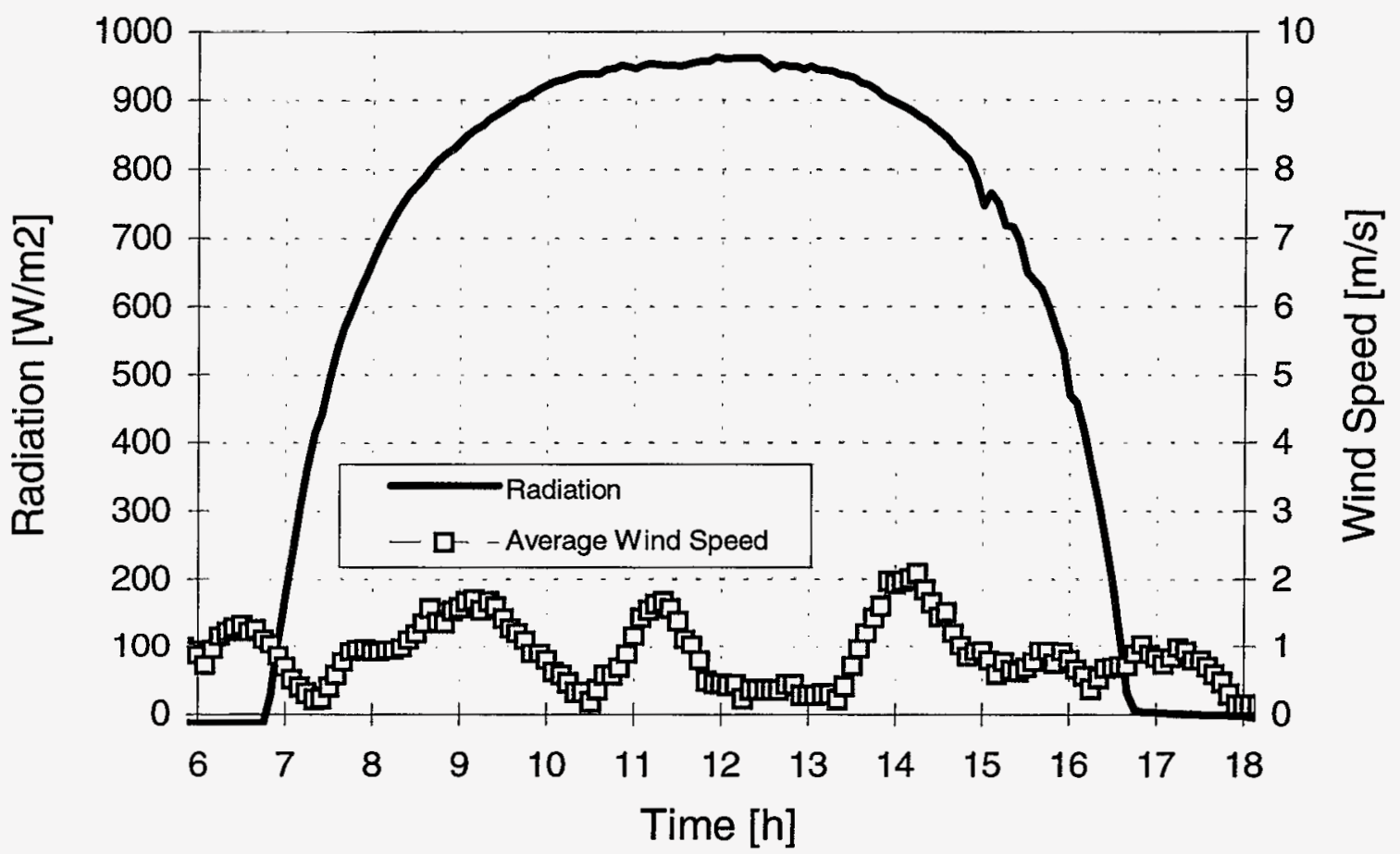

Figure V-4. Insolation and wind speed during a clear winter day.

Table V-3. Case Definitions for a Winter Day

\begin{tabular}{|l|l|}
\hline \multicolumn{1}{|c|}{ Case } & \multicolumn{1}{|c|}{ HCE/Field Conditions } \\
\hline W1 & $87.3 \%$ with vacuum \\
(base case) & $3.0 \%$ with air \\
& $7.5 \%$ bare (wind $1 \mathrm{~m} / \mathrm{s}=2.2 \mathrm{mph}$ ), \\
& $1.6 \%$ with defective coating \\
& $0.6 \%$ affected by cooling tower problem (5 SCAs out of service) \\
& $0.34 \%$ of mirrors broken, 100.0\% of SCAs tracking \\
& Reflectivity $90.5 \%$ \\
\hline W2 & Additional assumption that absorptivity of bare tubes is reduced to $75 \%$ \\
& (degradation) \\
\hline W3 & Stronger wind $(2 \mathrm{~m} / \mathrm{s}=4.4 \mathrm{mph})$ \\
\hline
\end{tabular}




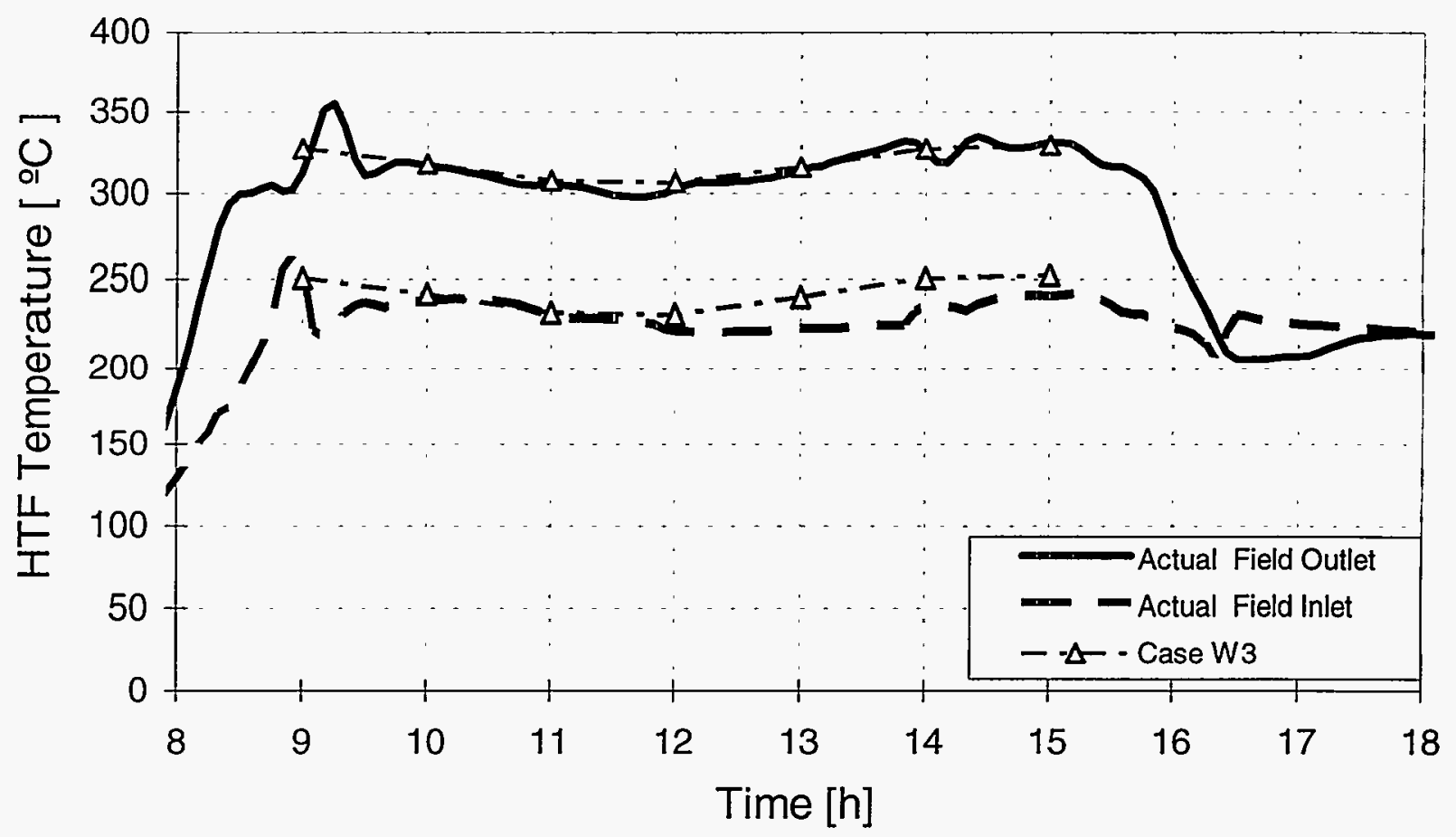

Figure V-5. HTF temperatures during a clear winter day.

\section{Summary}

In the calculations, a solar field performance equation is included that was developed out of measurement results of an LS-2 collector tested at SNL (Appendix A). The model results are compared with the real plant behavior for a winter day and a summer day.

The comparison with the real plant conditions shows that there is still a lack of information concerning actual solar field conditions. It also seems that the heat losses of the solar field are underestimated because the results are a little too high on winter and summer days. Here it is likely that, in particular, the piping heat losses assumed in the calculations were too low. It may also be that the optical efficiency of the solar field was worse than estimated, which is logical, because SNL's single trough was aligned very well compared with the troughs in the solar field. 


\section{Appendix W HOURLY PERFORMANCE MODEL}

The electric output and thermal performance of the Kramer Junction, California, plants are monitored on an hourly basis, comparing actual production against predicted production based on measured solar radiation data. This process is complicated by the difficulties of properly characterizing the plant performance parameters to be used in the model, e.g., average mirror reflectivity, status of the solar field, efficiency of the power block, and other such variables. Intelligent use of the model allows the plant supervision to identify systems that are operating below design levels, and consequently to plan corrective measures. Another critically important part of the modeling process is to calculate the amount of natural gas that can be used during the year. Federal Energy Regulatory Commission (FERC) rules for operation of these plants base the level of natural gas use for a given year on the thermal output of the solar field during that year. Thus, the plants start out a year making gas-use decisions based on their experience and common sense, and make final adjustments as the year comes to a close. The most important issue in this regard is the availability of natural gas to meet summer peak loads, and this is where careful modeling and well-planned gas-use strategies become crucial.

Performance and gas-use models (GUMs) are at the core of this process. However, it has long been recognized that the present models contained insufficient flexibility to properly model the plants, and that integration of the GUM with the performance model would be beneficial.

Moreover, the existing hourly models were developed by LUZ International Ltd. and backed by only limited documentation, making it difficult for the site staff to make changes or understand all the subtleties contained in the algorithms.

\section{KJCOC Performance Model}

To overcome the shortcomings of the LUZ model and the GUM, the KJC Operating Company (KJCOC) performance model was developed. It was developed to provide a single tool incorporating performance projection, development of the proper gas-use strategy to maximize revenues, and monitoring of performance loss mechanisms to prioritize operation and maintenance targets. This new model is also an hourly (or shorter time-step) simulation of a solar electric generating system (SEGS) plant. It was developed for the Windows environment using the Delphi programming tool and features a user-friendly design. A primary goal of the development of the $\mathrm{KJCOC}$ model was to acquire a tool to assist in the operation of a real power plant, not a research project. The financial results are ultimately the most important result of plant operation and must be maximized. This new model is described in the sections that follow.

The basis for the development of the KJCOC model was the LUZ model with the same algorithms used for steam production, electricity production, and available gas. The program flow for the KJCOC model is shown in Figure W-1. The various program sections are described in further detail below.

\footnotetext{
'This section is an excerpt from R. Nelson and R. Cable, The KJC Plant Performance Model - An Improved SEGS Plant Simulation, proceedings of the ASME/JSME/JSES International Solar Energy Conference, April 11-14, 1999, Maui, Hawaii.
} 


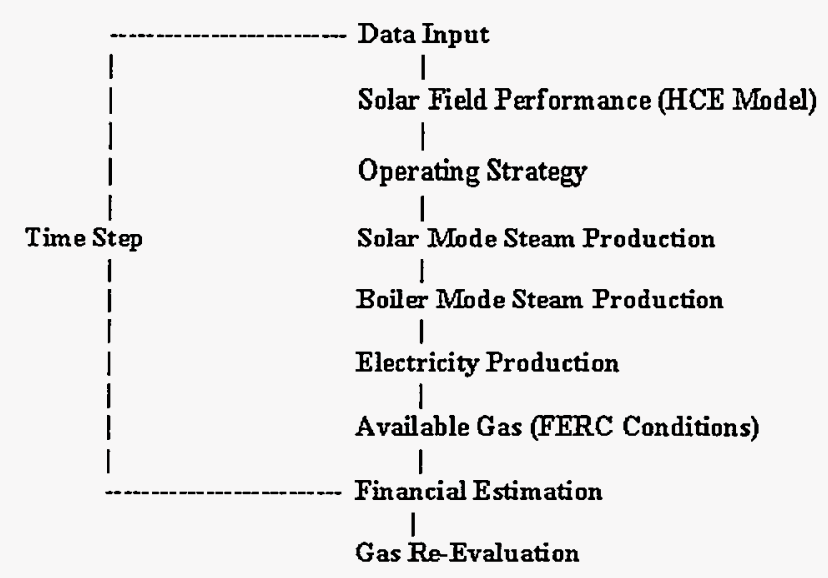

Figure W-1. KJCOC model flow diagram.

\section{Data Input}

In marked contrast to the LUZ model, all model parameters are easily viewed and changed by the user. All user inputs are organized on a tabbed notebook interface. In addition to the 150 input parameters of the LUZ model, the user can specify solar field conditions including inlet and outlet temperatures on a monthly basis, instead of using constant temperatures year round. In addition, the user can easily enter solar field conditions and can define new heat collection element (HCE) absorber types and specifications. Hourly or shorter time-step meteorological data are read from a user selectable data file. By default, the modified typical meteorological year data from the LUZ model are used.

A screen shot of the KJCOC model user interface is shown in Figure W-2.

\section{Solar Field Performance}

To improve the calculation of solar field performance, the HCE heat transfer model was improved based on results of the HCE testing described in Appendix A.

\section{Operating Strategy}

The model uses the time-of-use rate period to determine plant operation. Because power can be sold at a greater price, natural gas is only burned during on-peak and mid-peak periods. It is therefore important to make sure that the model burns all allowable gas during these periods. The operating strategy determines plant operation mode: solar mode, boiler mode, and hybrid mode. In solar mode, all steam is generated from heat from the solar field. In boiler mode, the boiler provides all available steam. In hybrid mode, the solar field provides part load steam, and the boiler is operated to bring the steam flow to full capacity.

\section{Solar and Boiler Steam Generation}

The steam generation section of the KJCOC model turns the output of the solar field into steam to operate the turbine. If heat is available from the solar field, the model converts that heat to steam using the steam generator efficiency and the enthalpy change. The enthalpy change is calculated as a function of solar mode steam-flow fraction. The steam-flow fraction is the current flow divided by the maximum possible flow. 


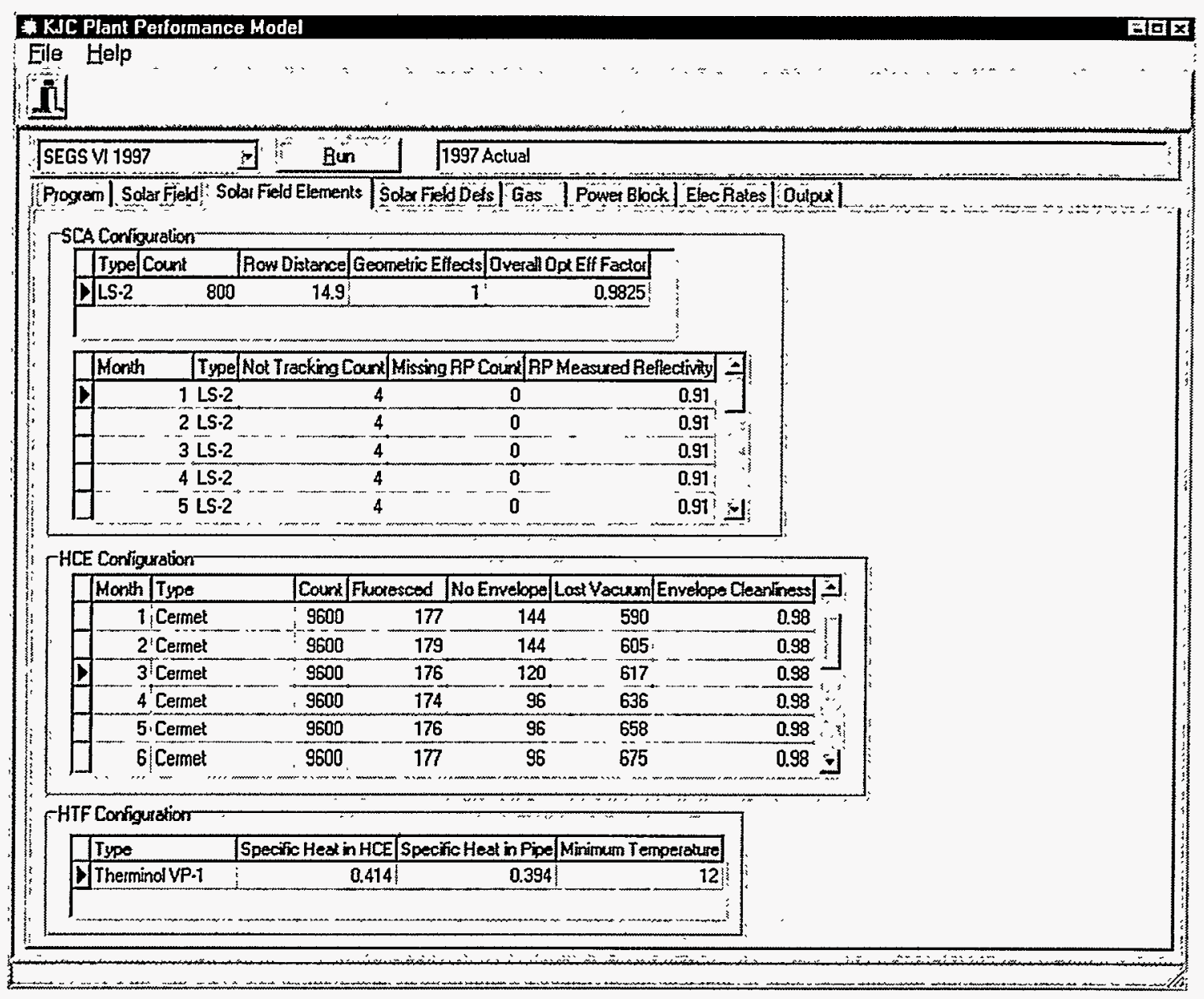

Figure W-2. KJCOC model user interface.

In solar mode, all steam is generated from heat from the solar field and the plant is operated at part or full load depending on the solar steam flow. If gas is available and the solar steam load is less than maximum, the plant is operated in hybrid mode with the boiler filling in steam to full load. If gas is available, but solar steam is not, then the plant is operated in boiler mode with the boiler operated to produce steam at full load. If the boiler is operated, the program accounts for natural gas use.

\section{Gross Electricity Production}

If there is sufficient solar steam, or the boiler is operating, the turbine operates to produce electricity. The model calculates the turbine efficiency differently for SEGS III through V and SEGS VI and VII. For SEGS III through V, the efficiency is calculated as a function of both pressure (a user input) and plant load. For SEGS VI and VII, the efficiency is a function of plant load only. The pressure calculation is based on efficiency versus pressure curves while the load calculation is from efficiency versus load curves, both from the turbine manufacturer.

\section{Parasitic Energy}

Parasitic energy loads are calculated for the power block, balance of plant, solar field, plant startup, and a constant fixed load that represents miscellaneous equipment. Power block, balance of plant, and solar field parasitic energies are calculated as a function of plant operating mode 
(solar, boiler, and hybrid) and fractional plant load. Plant startup energy is a fixed value depending on whether startup is warm or cold. The constant load does not change throughout the day.

\section{Natural Gas Use}

Natural gas is used to operate the boiler. As described in the Gas Reevaluation section, gas use is limited by the FERC requirements. Natural gas use must therefore be restricted. The model checks to see that gas is available for the current hour. Gas is used to operate the boiler and to heat the heat transfer fluid (HTF) during cold periods to keep the HTF warm. The minimum temperature is a user-input value that should be above the HTF freezing point of $54^{\circ} \mathrm{F}$. Nighttime losses are calculated using the same HCE model algorithms as daytime heat gains.

\section{Financial}

The model calculates electricity sales, electricity purchases, and natural gas purchases based on the appropriate utility rate for the time-of-use rate period. Electricity sales have three components: energy payments, capacity payments, and energy bonus payments. Energy payments are based on the kilowatt-hours $(\mathrm{kWh})$ sold. Capacity payments are based on the monthly energy production as a fraction of plant capacity. Energy bonus payments are added for energy production above the plant rating. Electricity purchases are energy charges. Gas purchases are based on the gas used.

These cash flows are summed to allow the financial implications of different plant operating strategies to be seen.

\section{Gas Reevaluation}

The model actually runs a number of times throughout the year. The first run-through assumes that there is an unlimited supply of natural gas to burn. This is not actually true because FERC regulations limit the use of natural gas to $25 \%$ of the energy input to the plant. Therefore, gas use must be allocated to be burned during the most valuable periods. For each time-of-use rate period, the user specifies monthly priority values and capacity targets. The second run of the model uses those priorities to restrict gas use during low priority periods and limit gas use to the capacity target. Starting with the off-peak period, the model removes available gas from the lowest priority months. If there is still excess gas use, the model removes gas from mid-peak (again starting with the lowest priority months) and then finally from on-peak. After the second run, this adjustment process is repeated and a third run is completed. If the user chooses, a final monthly adjustment is performed to exactly match capacity targets.

\section{KJCOC Model Operation}

The KJCOC model has three program operation modes: project mode, combine mode, and losses mode. Each is described in detail below.

\section{Project Mode}

In project mode, the model simulates plant performance using base case meteorological data. Plant operating parameters and solar field conditions are defined using model inputs. This mode 
is mainly used for budget purposes of future years or for determining the effects of maintenance practices (for example, evaluating the replacement of HCEs and reflector panels). Project mode can also be operated as a "solar-only" run, which does not allow boiler operation. An example of the annual report for a project mode run is shown in Figure W-3.

\section{Combine Mode}

Historical data and projection data are combined to simulate plant operation for the remainder of a calendar year. Historical data are read from the KJCOC operations database, and the KJCOC model calculates performance for the remainder of the year. In this mode, regular adjustments can be made to plant operations to ensure optimal gas use. This does the job of the old GUM. As described in the Gas Reevaluation section above, meeting the FERC 25\% limit is critical to plant operation. This mode allows gas use to be monitored throughout the year. Similar to the annual report shown in Figure W-3 for project mode, combine mode would show a similar report. However, with combine mode, all actual data (1 to 12 months) would be shown instead of the projected data.

\section{Losses Mode}

In losses mode, solar field losses are calculated for each solar field element for a month. This mode incorporates a more rigorous version of the analysis formerly performed with the solar performance loss model. For a month of historical data, the model is run nine times to evaluate the performance losses associated with each potential solar field flaw. The first run is an engineering projection using plant parameters as entered in the KJCOC model. The second run uses actual plant parameters from the KJCOC operations database. Each additional run changes one parameter to the actual value from the operations database while keeping the other parameters the same as the engineering projection. The performance impact of each parameter is then calculated in megawatt-hours (MWh). An example of the output from losses mode can be seen in Figure W-4. The report columns are the actual plant production, the calculated engineering projection using actual meteorological data, the difference between the two (production shortfall), the shortfall attributed to solar field losses, and the shortfall from each individual solar field element.

\section{Comparisons}

As shown Table W-1, the KJCOC performance model predicted the performance of SEGS VI for 1997 better than both the LUZ performance model and the GUM. The GUM is expected to project better than the LUZ performance model based on the adjustment factors developed for the GUM, as discussed previously. All projection models tend to estimate high because of the unpredictability of actual forced outages. For the year, the KJCOC performance model predicted $1.6 \%$ over actual, where the GUM was $3.9 \%$ higher, and the LUZ model was $13.1 \%$ higher than actual. On a daily basis, the LUZ performance model and the KJCOC performance model are compared in Figure W-5 for the month of July. As shown, the KJCOC performance model models actual conditions much better than the LUZ performance model. It should be noted that like inputs (optical efficiencies) were used in this comparison. The major reason the KJCOC performance model models closer to actual is its ability to deal with actual HCE conditions better. 


\section{KJC Plant Performance Model \\ SEGS VI 1999 Proj \\ 1999 Projection Run}

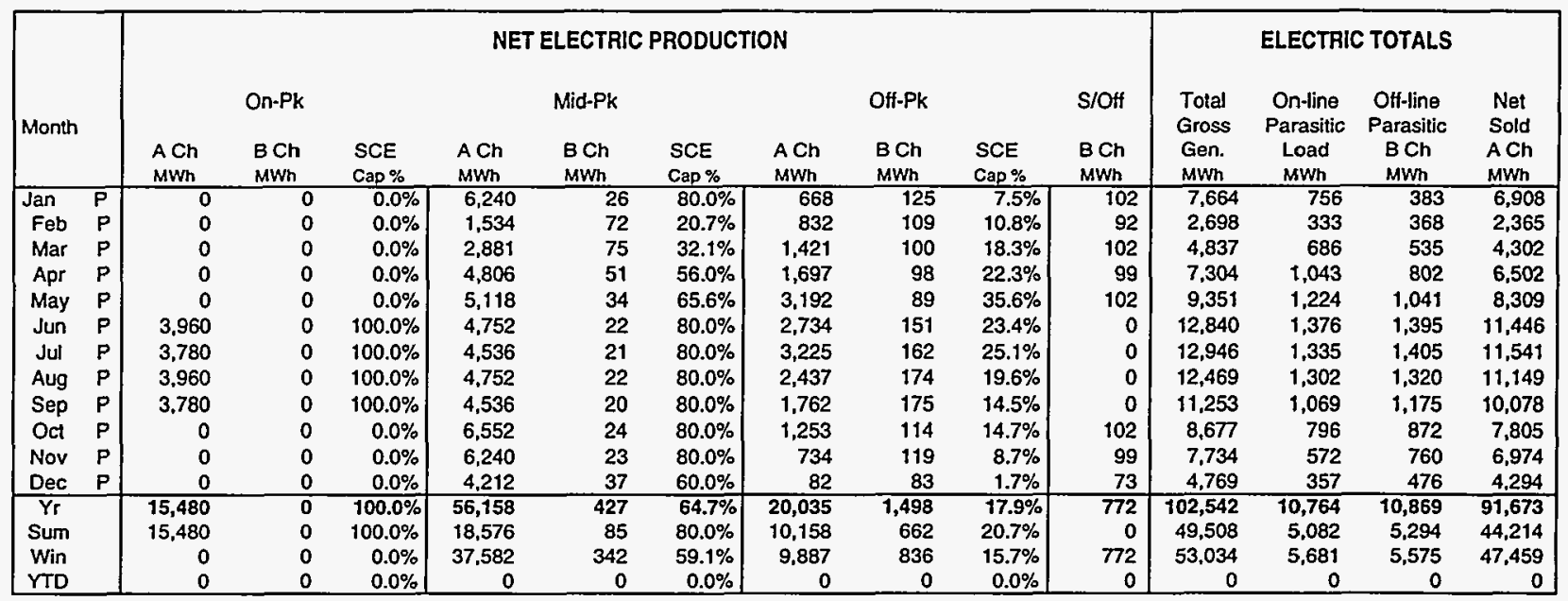

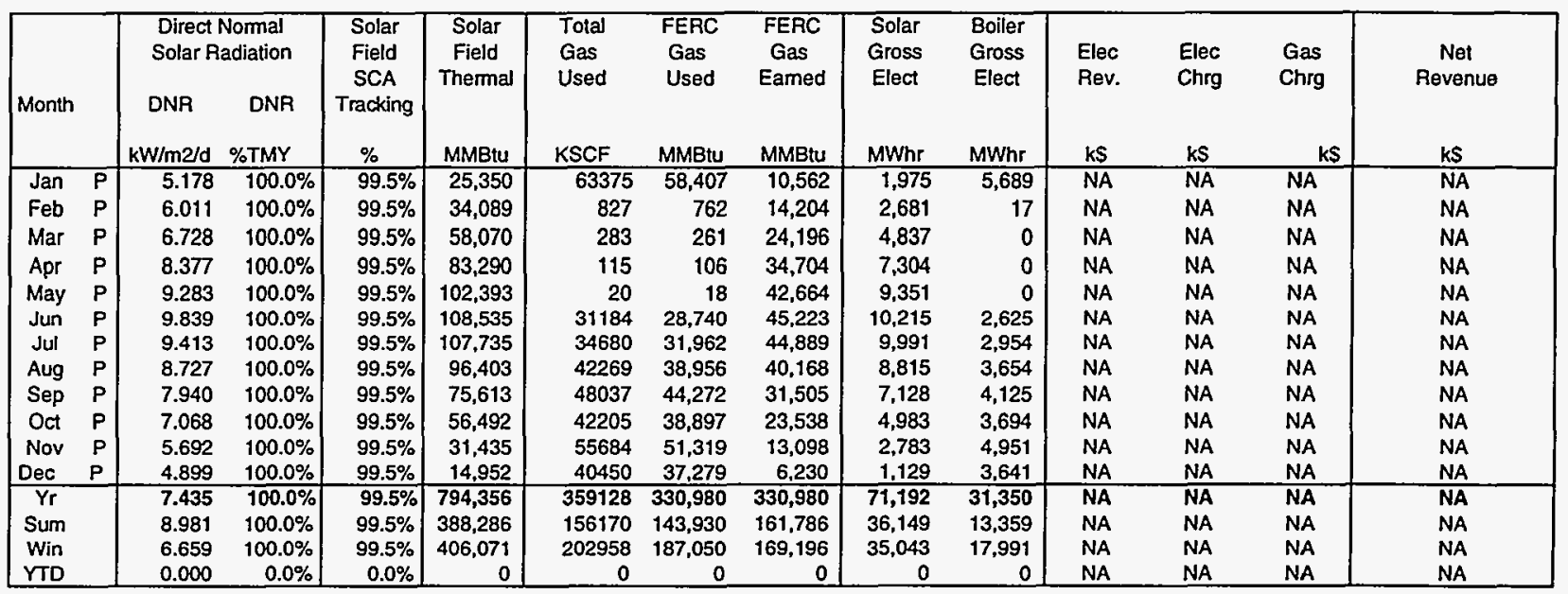

FERC $\Rightarrow \quad 25.00 \%$

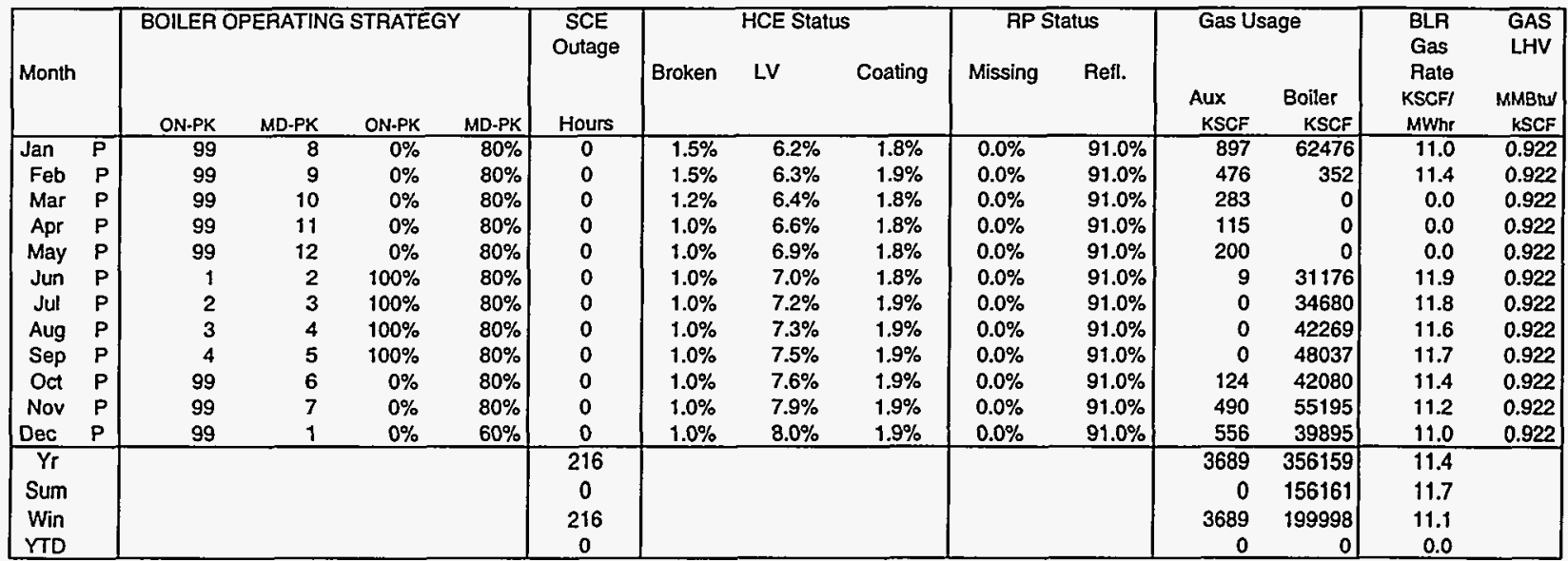

Figure W-3. Annual report for a project mode run. 


\section{Conclusions}

By integrating a three-tiered modeling process into a single program, the $\mathrm{KJCOC}$ performance model has greatly simplified the modeling process at the Kramer Junction SEGS. In addition, now that more detail has been added to the new $\mathrm{KJCOC}$ performance model, a more realistic approach can be taken for modeling actual conditions of the plants. This is not only important for the day-to-day quantification of losses, but a much needed tool for projecting performance in future years. In addition to a quantitative improvement in the accuracy of the modeling process, the $\mathrm{KJCOC}$ model has made a qualitative improvement in the ease and frequency of modeling. For the plant operators, this tool is expected to provide many of the answers to cost/benefit analysis that are becoming more important as the market for electricity becomes more competitive.

\begin{tabular}{|c|c|c|c|c|c|c|c|c|c|c|}
\hline & & & & $\begin{array}{r}\text { KJC Lo } \\
\text { EGS VI } 195\end{array}$ & $\begin{array}{l}\text { st Solar A } \\
97 \text { Losses }\end{array}$ & $\begin{array}{l}\text { inalysis } \\
- \text { April } 19\end{array}$ & & & & \\
\hline Production & $\begin{array}{c}\text { Actual } \\
\text { Production }\end{array}$ & $\begin{array}{l}\text { Engineering } \\
\text { Projection }\end{array}$ & $\begin{array}{l}\text { Production } \\
\text { Shortfall }\end{array}$ & $\begin{array}{l}\text { Solar Field } \\
\text { Shortfall }\end{array}$ & $\begin{array}{l}\text { Availability / } \\
\text { Tracking }\end{array}$ & $\begin{array}{c}\text { RP } \\
\text { Reflectivity }\end{array}$ & $\begin{array}{l}\text { Broken } \\
\text { Envelope }\end{array}$ & $\begin{array}{c}\text { HCE } \\
\text { Lost } \\
\text { Vacuum }\end{array}$ & Fluoresced & $\begin{array}{c}\text { Missing } \\
\text { RPs }\end{array}$ \\
\hline Day & Mwh & Mwh & MWh & MWh & MWh & MWh & MWh & MWh & MWh & MWh \\
\hline 1 & 104 & 121 & 17 & 16 & 2 & 10 & 1 & 1 & 2 & 0 \\
\hline 2 & 160 & 210 & 50 & 36 & 4 & 25 & 2 & 2 & 3 & 0 \\
\hline 3 & 43 & 69 & 26 & 11 & 1 & 6 & 2 & 1 & 1 & 0 \\
\hline 4 & 252 & 309 & 57 & 41 & 4 & 28 & 3 & 2 & 4 & 0 \\
\hline 5 & 186 & 202 & 16 & 24 & 2 & 15 & 2 & 2 & 3 & 0 \\
\hline 6 & 193 & 214 & 21 & 26 & 2 & 16 & 3 & 2 & 3 & 0 \\
\hline 7 & 247 & 293 & 46 & 40 & 2 & 26 & 5 & 2 & 4 & 1 \\
\hline 8 & 262 & 319 & 57 & 41 & 4 & 28 & 3 & 2 & 4 & 0 \\
\hline 9 & 284 & 333 & 49 & 47 & 4 & 32 & 4 & 2 & 5 & 0 \\
\hline 10 & 274 & 314 & 40 & 45 & 4 & 28 & 6 & 2 & 4 & 1 \\
\hline 11 & 298 & 362 & 64 & 50 & 4 & 35 & 4 & 2 & 4 & 1 \\
\hline 12 & 283 & 324 & 41 & 39 & 4 & 25 & 4 & 2 & 4 & 0 \\
\hline 13 & 304 & 342 & 38 & 41 & 4 & 27 & 4 & 2 & 4 & 0 \\
\hline 14 & 280 & 326 & 46 & 39 & 4 & 25 & 4 & 2 & 3 & 1 \\
\hline 15 & 291 & 331 & 40 & 41 & 4 & 26 & 5 & 2 & 3 & 1 \\
\hline 16 & 304 & 345 & 41 & 43 & 4 & 30 & 4 & 2 & 3 & 0 \\
\hline 17 & 308 & 337 & 29 & 32 & 4 & 15 & 6 & 2 & 4 & 1 \\
\hline 18 & 263 & 289 & 26 & 27 & 2 & 12 & 5 & 3 & 4 & 1 \\
\hline 19 & 178 & 182 & 4 & 17 & 2 & 5 & 4 & 2 & 3 & 1 \\
\hline 20 & 130 & 134 & 4 & 10 & 1 & 3 & 3 & 1 & 2 & 0 \\
\hline 21 & 60 & 14 & -46 & 5 & 2 & 2 & 1 & 0 & 0 & 0 \\
\hline 22 & 204 & 206 & 2 & 14 & 2 & 4 & 3 & 2 & 3 & 0 \\
\hline 23 & 112 & 332 & 220 & 210 & 195 & 3 & 7 & 3 & 2 & 0 \\
\hline 24 & 327 & 343 & 16 & 19 & 4 & 3 & 6 & 2 & 3 & 1 \\
\hline 25 & 334 & 347 & 13 & 23 & 4 & 5 & 7 & 3 & 2 & 2 \\
\hline 26 & 355 & 368 & 13 & 17 & 4 & 5 & 4 & 2 & 2 & 0 \\
\hline 27 & 206 & 209 & 3 & 10 & 2 & 2 & 2 & 2 & 2 & 0 \\
\hline 28 & 197 & 208 & 11 & 11 & 2 & 2 & 3 & 2 & 2 & 0 \\
\hline 29 & 239 & 255 & 16 & 14 & 2 & 5 & 3 & 2 & 2 & 0 \\
\hline 30 & 354 & 373 & 19 & 17 & 4 & 6 & 3 & 2 & 2 & 0 \\
\hline Total & 7032 & 8011 & 979 & 1007 & 283 & 454 & 113 & 59 & 87 & 11 \\
\hline
\end{tabular}

Figure W-4. KJCOC lost solar analysis. 
Table W-1. Monthly/Annual Model Comparison

\begin{tabular}{lccccccc} 
& $\begin{array}{c}\text { Actual } \\
\text { Gross }\end{array}$ & $\begin{array}{c}\text { Luz } \\
\text { Gross }\end{array}$ & $\begin{array}{c}\text { GUM } \\
\text { Gross }\end{array}$ & $\begin{array}{c}\text { KJC } \\
\text { Gross }\end{array}$ & $\begin{array}{c}\text { Luz vs } \\
\text { Acual }\end{array}$ & $\begin{array}{c}\text { GUM vs } \\
\text { Actual }\end{array}$ & $\begin{array}{c}\text { KJC vs. } \\
\text { Actual }\end{array}$ \\
Jan & 2 & 0 & 0 & 0 & $0.0 \%$ & $0.0 \%$ & $0.0 \%$ \\
Feb & 2249 & 2812 & 2482 & 2023 & $125.0 \%$ & $110.4 \%$ & $90.0 \%$ \\
Mar & 6352 & 7820 & 7848 & 6670 & $123.1 \%$ & $123.6 \%$ & $105.0 \%$ \\
Apr & 7032 & 8252 & 7153 & 7189 & $117.3 \%$ & $101.7 \%$ & $102.2 \%$ \\
May & 9413 & 10072 & 8717 & 8768 & $107.0 \%$ & $92.6 \%$ & $93.1 \%$ \\
Jun & 9870 & 10523 & 9659 & 9877 & $106.6 \%$ & $97.9 \%$ & $100.1 \%$ \\
Jul & 10363 & 10703 & 10024 & 10354 & $103.3 \%$ & $96.7 \%$ & $99.9 \%$ \\
Aug & 9039 & 9908 & 9420 & 9014 & $109.6 \%$ & $104.2 \%$ & $99.7 \%$ \\
Sep & 6373 & 7563 & 6538 & 6652 & $118.7 \%$ & $102.6 \%$ & $104.4 \%$ \\
Oct & 5517 & 6835 & 6599 & 5980 & $123.9 \%$ & $119.6 \%$ & $108.4 \%$ \\
Nov & 2089 & 2380 & 1953 & 2296 & $113.9 \%$ & $93.5 \%$ & $109.9 \%$ \\
Dec & 1720 & 2317 & 2362 & 2337 & $134.7 \%$ & $\mathbf{1 3 7 . 3 \%}$ & $\mathbf{1 3 5 . 9 \%}$ \\
\hline \hline Year & 70019 & 79184 & 72755 & 71160 & $113.1 \%$ & $103.9 \%$ & $101.6 \%$ \\
Summer & 35645 & 38697 & 35641 & 35896 & $108.6 \%$ & $100.0 \%$ & $100.7 \%$ \\
Winter & 34374 & 40487 & 37114 & 35263 & $117.8 \%$ & $108.0 \%$ & $102.6 \%$
\end{tabular}

Monthly Model Comparison

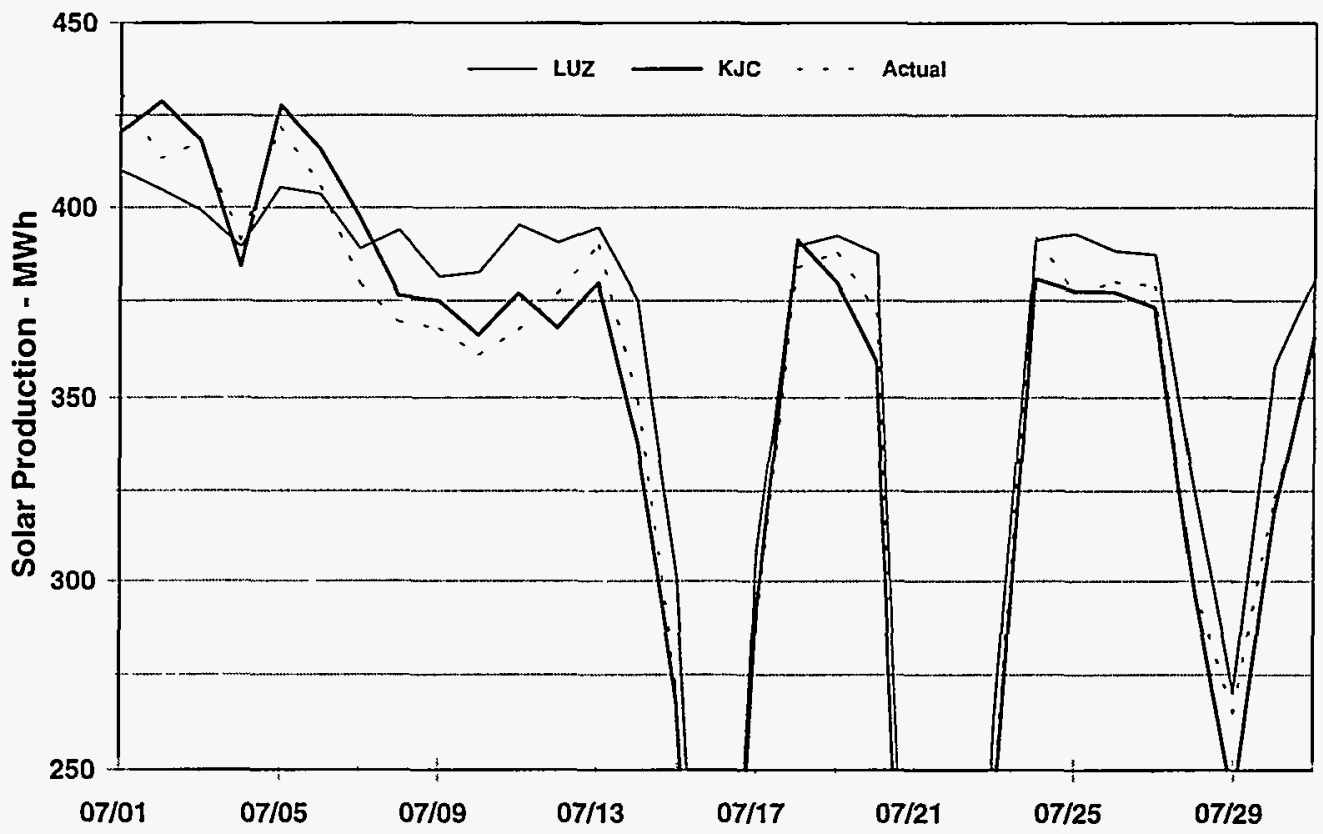

Figure W-5. Daily/monthly model comparison. 


\section{Appendix X \\ ON-Line PeRformance Monitoring ANd Optimization}

\section{Background}

Optimization of plant performance depends on effective and efficient integration of the solar field with the power block. Examples of this include matching the solar field outlet conditions to the power block and coordinating the production of steam by the solar field with the ability of the power block to use that steam. In the winter season, when steam production by the solar field is below peak and the outlet temperature drops off, the operator has a choice of producing steam at a lower temperature and pressure but at a higher flow rate than would be possible at higher steam conditions. Which choice will optimize net power output? In summer, the solar field can produce steam quickly in the morning, but proper warm-up of the turbine and steam lines often requires either discarding steam or defocusing collectors. Both measures are inefficient and costly. Can other steps be taken to reduce this waste? This task aims to provide some answers to these and other questions by on-line monitoring of the plant heat rate and power output to observe the real-time effects of operator decisions and to provide data for engineering changes where required or appropriate.

This task focused on the development and implementation of an On-line Performance Monitoring (OPM) program to track plant heat rate, power output, equipment efficiencies, and other key parameters on Solar Electric Generating Systems (SEGS) VI and VII. Predictive "what if" capability will allow the operators and plant engineers to determine the probable results of proposed changes in key operating parameters. Once operating conditions are changed, the OPM system will show the results of the action(s). An important first step was to ensure that reliable and complete instrumentation existed or was installed to provide all the necessary plant operating points, e.g., pressures, temperatures, and flows.

\section{OPM Software Development}

There are a number of vendors offering OPM software. After evaluation of existing products and firms, a subcontract was awarded to Black \& Veatch (B\&V) to develop an OPM system for SEGS VI and VII. The decision was based on B\&V's existing and well-tested OPM product, which uses a modular, traditional modeling approach rather than a more empirical method. This approach provided flexibility and power, and allowed a systematic addition of a solar field model. Other factors affecting the decision were the extensive experience of B\&V in power plant design and OPM, confirming the expectation of strong technical support. The basic features of the B\&V OPM system are the following:

- User Interface - the user's access to data and information. About 30 view screens were established through extensive interaction between B\&V, plant operating personnel, and plant engineers. The overall screen for SEGS VI is shown in Figure X-1.

- Data Enhancement Module - ensures the integrity of the data set received from the data acquisition system. 


\section{- Calculation Modules}

- Feedwater Heater Performance

- Condenser Performance

- Turbine-Generator Performance (including heat rate)

- Cooling Tower Performance

- Solar Field Performance

- Controllable Losses

- Integrated Plant Model - The Integrated Plant Model (IPM) is the tool that permits predictive or "what if" scenarios to be performed. Using this tool, the operator can quantify effects on performance, such as heat rate, due to changes in operator controllable parameters, such as solar field outlet temperature. The IPM is divided into four major subsystems: solar field, solar boiler, gas boiler, and steam turbine. The model also allows for different plant operating modes, i.e., gas-only, solar-only, and hybrid.

- Solar Field Model - The solar field model is a simplified model of the solar field to predict its output as a function of solar radiation and operational condition, including mirror reflectivity and status of the field heat collection elements. This in turn allows calculation of the thermal efficiency of the solar field. This model is fully described in Appendix V.

The OPM model was implemented in 1996.

\section{Results}

At the date of this writing, the control room operators had not been fully trained to use OPM. Only one training class had been offered and that training was not adequate for the operators to become comfortable with the system. The engineering staff was beginning to use it to retrieve performance data to help them reconstruct causes of plant trips. Thus, we are unable to evaluate the usefulness of this tool to help optimize plant performance. 


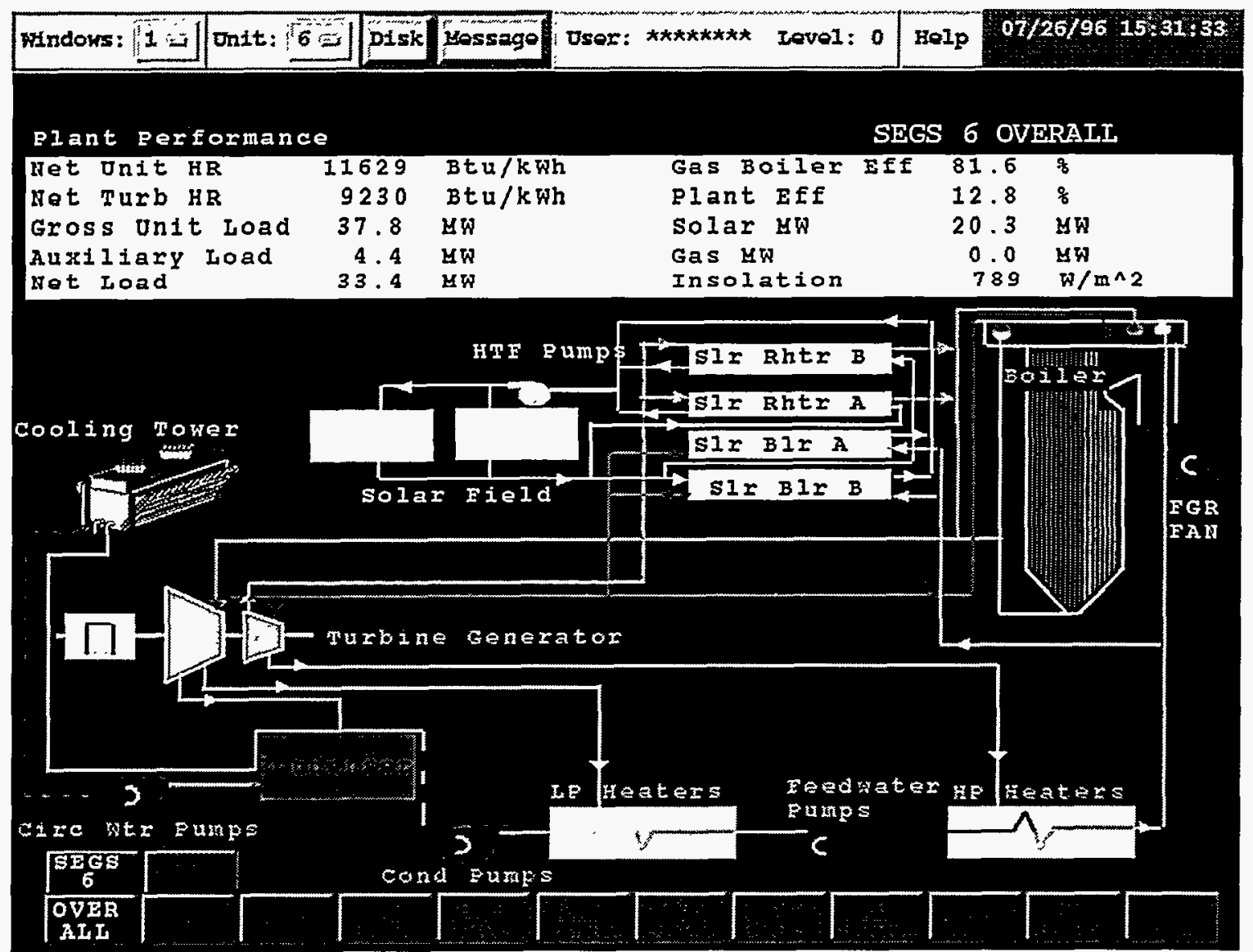

Figure X-1. Overall OPM view screen for SEGS VI. 
This page intentionally left blank. 


\section{Appendix $Y$ \\ WeATHER Station Data MANAGEMENT}

Each of the five solar electric generating system (SEGS) plants has a weather station that gathers data on ambient air temperature, wind speed and direction, and direct-normal irradiance.

Selected stations have pyranometers for global irradiance data. The data are collected as 5- or 10-minute averages of one-second samples. The data had proven to be somewhat inconsistent between plants, leading to the desire for better equipment and methods.

A central weather station with improved software was designed to improve the quality and consistency of the weather data for the entire site. The new weather station includes sensors for direct-normal, diffuse, and global solar irradiance; wind speed; wind direction; barometric pressure; relative humidity; rain; and ambient air temperature. The remaining task was to develop software to control the collection, processing, and reporting of data.

The weather station equipment was installed in July 1995. Weather station software was developed by Augustyn + Co for data acquisition and processing. The Weather Station Software System has three main parts:

- DQMS (Data Quality Management System) - This system processes collects data.

- DataPipe - This program channels datalogger scans and averages to DQMS and DataView.

- DataView - This program makes real-time and historical graphs.

DQMS and DataPipe are controlled and maintained by the Weather Station operator. DataView can be used by anyone at KJC Operating Company with network access to view real-time or historical Weather Station data. Figure Y-1 shows the main elements of the system.

DataPipe is an independent, compiled program controlled by the Weather Station operator. DataPipe works in conjunction with DQMS, the Campbell Scientific PC208 software, and a program named CSI16COM.DLL, which is designed to communicate with Campbell Scientific dataloggers. DataPipe requires that a direct or continuous modem link is maintained between the computer on which it is running and the datalogger (either a Campbell 21X, CR10, or CR500). When so linked, DataPipe allows the operator to select one or more datalogger input storage locations for real-time retrieval and viewing through DataView. DataPipe also automatically detects when datalogger (final storage) output records are generated and automatically imports them into the proper DQMS data table.

DataView is a program that works with both $D Q M S$ and DataPipe. DataView allows the creation and viewing of customized graphs of "Real Time" data (through DataPipe) and "Historical" data (through DQMS). Multiple copies of DataView can be running at the same time on different computers in the network. DataView files may be placed in any directory, on the same machine running DataPipe, or on any other computer on the network. DataView graphs are highly configurable. The standard DataView screen is shown in Figure Y-2, as is a historical DataView graph. 
DQMS is designed to process time sequence numerical data collected from monitoring stations-either singly or in networks. It can handle changes to existing datalogger records and the addition of new records. The many features of DQMS are illustrated in Figure Y-3. Quality control tests are carried out on the data routinely. At the end of each day, DQMS processes the daily data using the National Renewable Energy Laboratory's quality control software, known as SERIQC, to look for missing data, out-of-range data, and data that fail comparative tests. In the event of data problems, flags notify the operator of bad data, but no automatic corrections are made by DQMS.

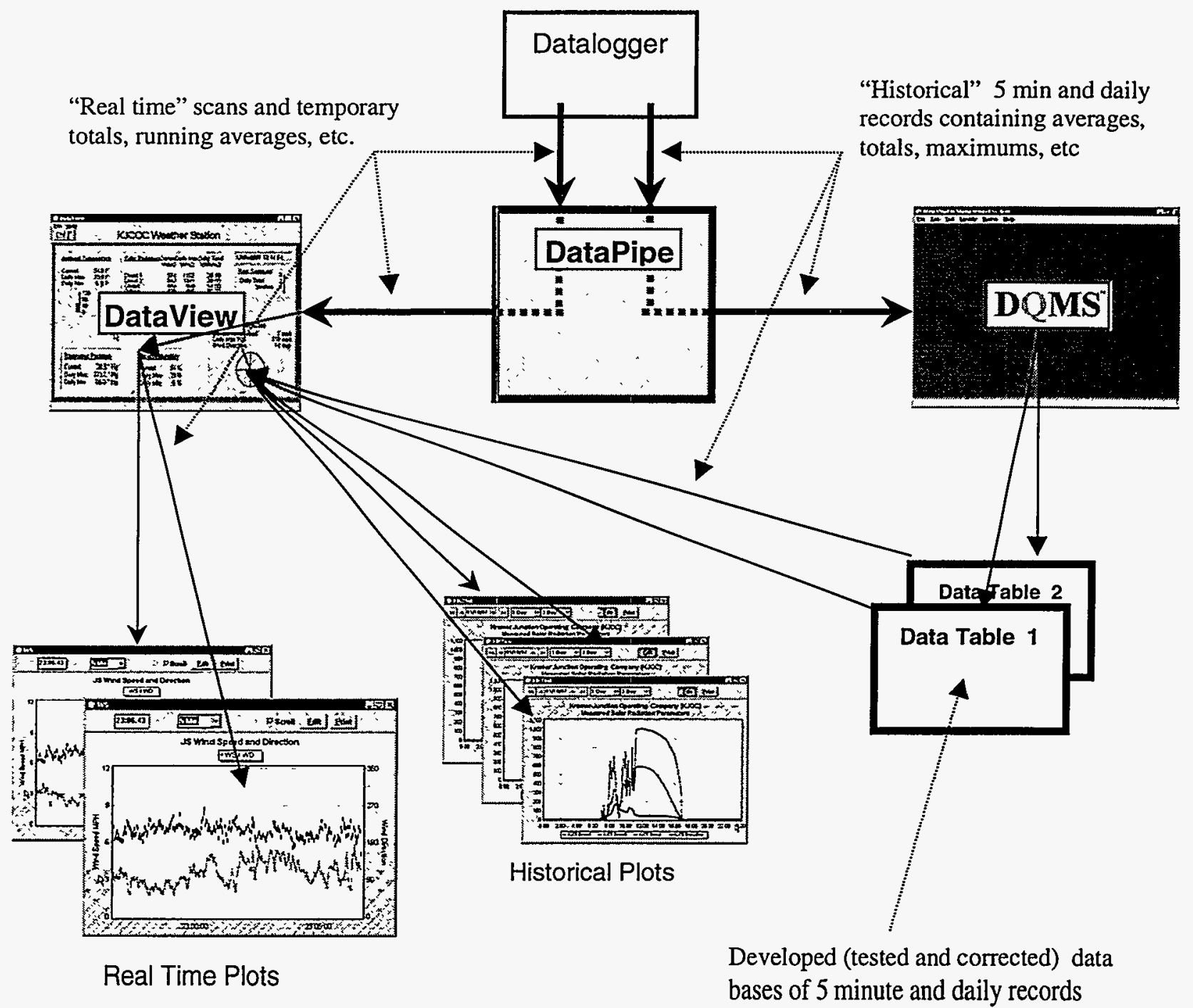

Figure Y-1. The main elements of the system. 


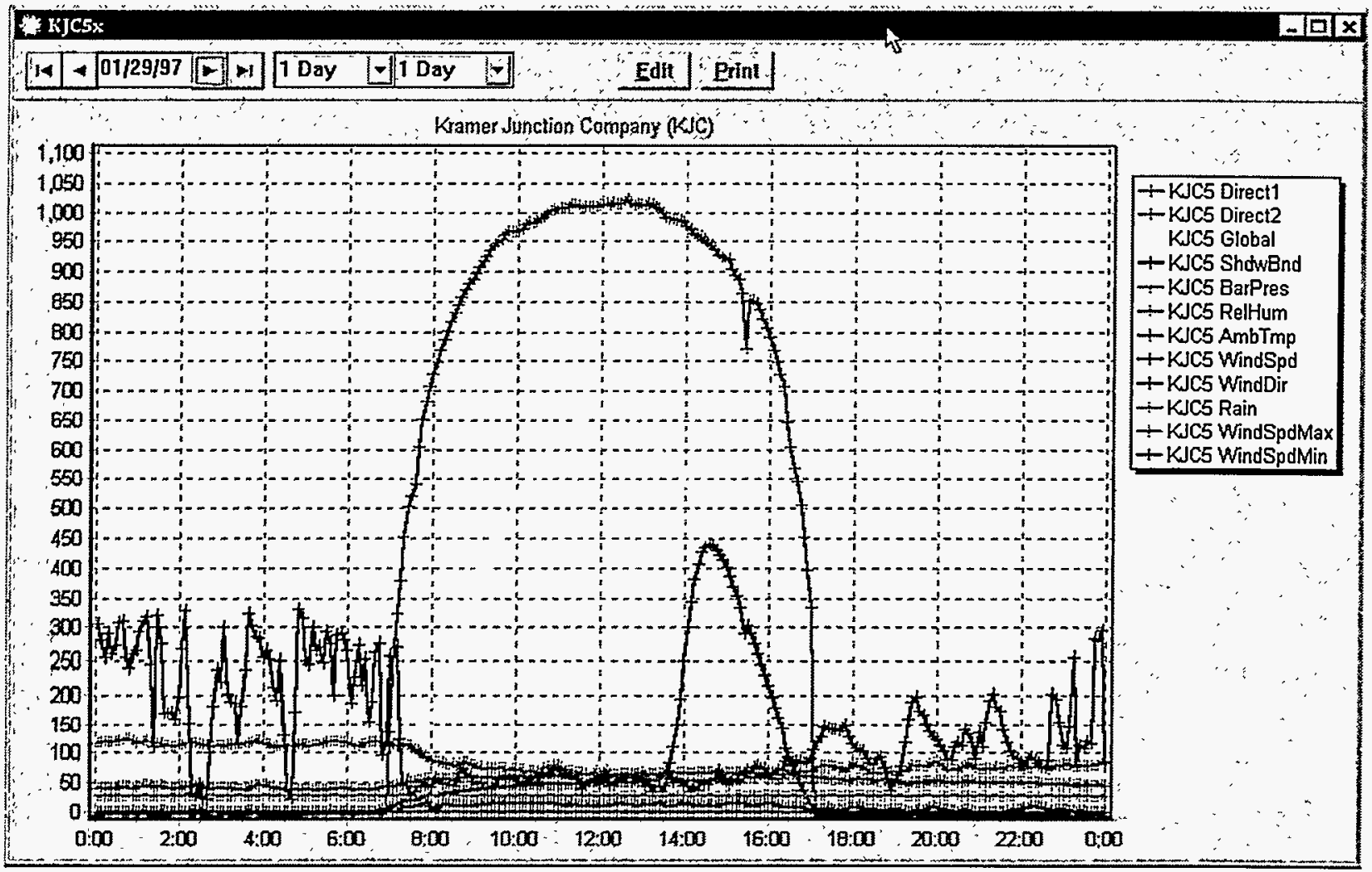

Figure Y-2. The standard DataView screen. 


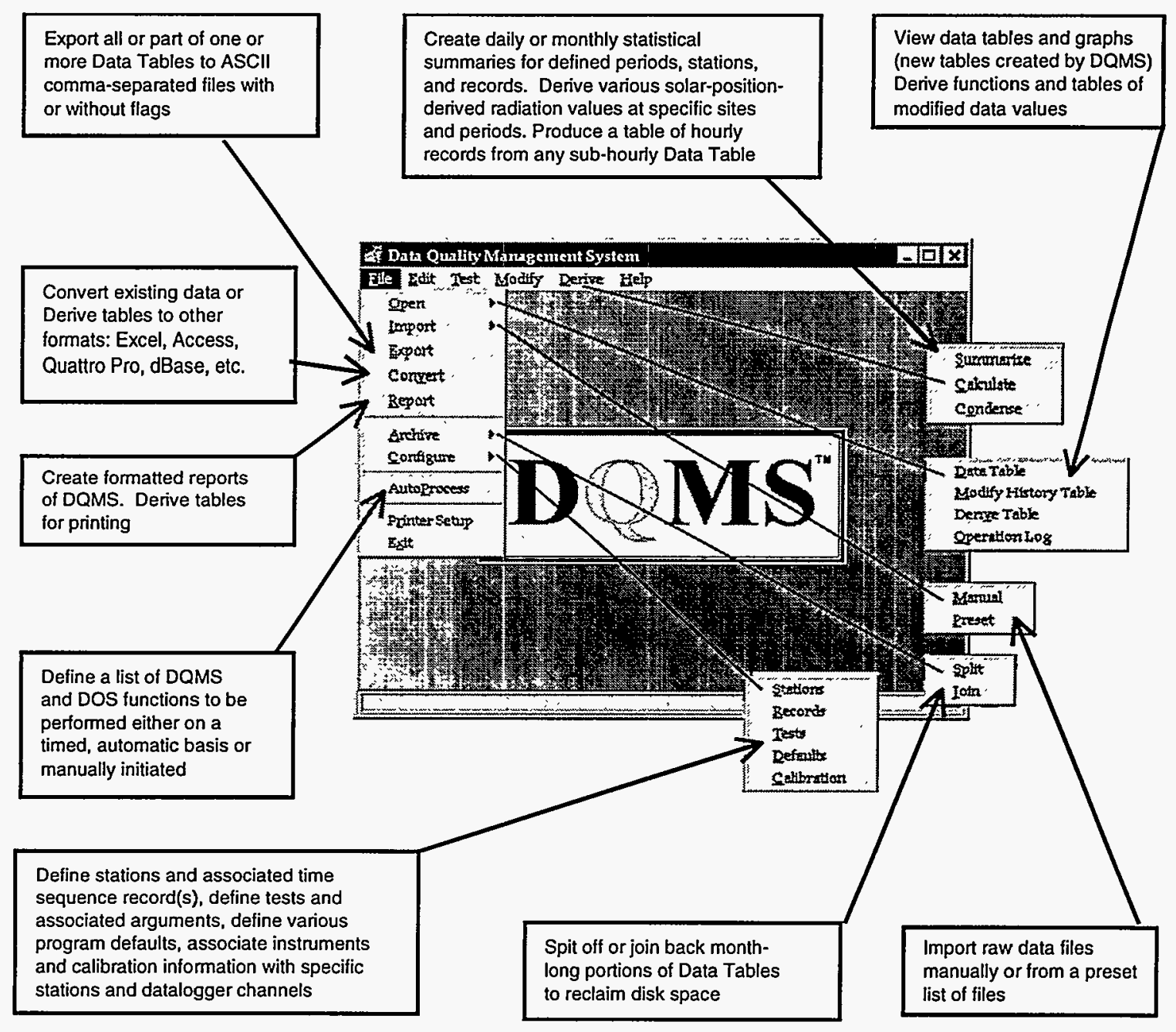

Figure Y-3. The DQMS features. 


\section{Appendix Z \\ FUGITIVE EMISSIONS}

\section{Background}

The heat transfer fluid (HTF) used in the solar field is a synthetic hydrocarbon chosen for its superior characteristics of heat capacity, flow, and chemical stability. It is a eutectic mixture of biphenyl (26\%) and diphenyl oxide (74\%). These compounds are included in the definition of polycyclic organic matter but are not polyaromatic hydrocarbons. The HTF is considered a hazardous material by the California regulatory definition, but is not a federally restricted material under the Resource Control and Recovery Act. It condenses readily at ambient conditions; therefore, gaseous concentrations are usually well below the permissible exposure level of approximately $1 \mathrm{ppm}$, as defined by the Occupational Safety and Health Administration. Vapor releases of HTF form visible vapor clouds, which either quickly settle or rise and disperse, depending on ambient conditions.

Each of Solar Electric Generating Systems (SEGS) III through VII contains approximately 100,000 gallons $\left(380,000\right.$ liters) of HTF. The maximum design operating temperature is $735^{\circ} \mathrm{F}$ $\left(390^{\circ} \mathrm{C}\right)$, with the corresponding vapor pressure of $135 \mathrm{psi}(9.2 \mathrm{bar})$. The maximum system pressure reaches about $600 \mathrm{psi}(41 \mathrm{bar}$ ) to pump the fluid through the solar fields. With these fluid conditions of HTF flowing through miles of pipe and thousands of fittings, there are bound to be some losses because of fugitive emissions and system breaches. These losses translate to hydrocarbon emissions, which are considered both volatile organic carbon compound and reactive organic gases. The purpose of this task was to identify and quantify fugitive emissions from the HTF at SEGS III through VII, and to determine whether these emissions constituted a significant percentage of annual HTF losses.

\section{Results}

A contract was awarded to AeroVironment to conduct a series of activities consisting of source inventory, sampling, quantification calculations, and recommendations for improvements. Measurements to estimate the quantity of fugitive emissions were made during the summer months of 1994.

Three measurement methods were used. The first method was based on the amount of HTF KJC Operating Company (KJCOC) added to each SEGS on an annual basis. The second method was the Environmental Protection Agency's (EPA's) average emission factor method. ${ }^{*}$ This method simply required inventorying the plumbing components and multiplying the component count by the EPA's emission factors for each type of plumbing component. The third method was the EPA leak rate/screening value correlation. ${ }^{*}$ This latter method has been found by the EPA and the Chemical Manufacturers Association to be the most accurate. The EPA's component leak rates were verified to be reasonable by actually measuring the leak rates on 24 components at $\mathrm{KJCOC}$; components were covered with bags and the entrapped air was drawn onto a Tenax trap for subsequent gas chromatography with flame ionization detection analysis.

\footnotetext{
"Chemical Manufacturers Association, Improving Air Quality: Guidance for Estimating Fugitive Emissions from Equipment, 1998.
} 
Hydrocarbon concentration measurements from more than 1,000 fittings at SEGS IV and VI were compiled. Factors from the EPA were used to convert these concentrations to mass emission rates. Inventories of potential leak points and pressure-cycle data were then used to estimate the annual fugitive losses from the SEGS III through VII sites. The results in Table Z-1 indicate an estimated annual fugitive loss of 4,100 pounds $(1,900 \mathrm{~kg})$, which is only about $0.08 \%$ of the HTF on site.

Inventory mass balances of the HTF system have indicated an overall fluid loss of about $1 \%$ annually. It appears that fugitive emissions from normal operating conditions contribute only a small portion of this loss. Other losses come from larger system leaks and system breaches. Such breaches are isolated and repaired immediately, but losses of fluid may be rapid and thus constitute most of the annual HTF loss.

Table Z-1. Hourly and Annual Fugitive Emissions for Each HTF Plumbing Component Category

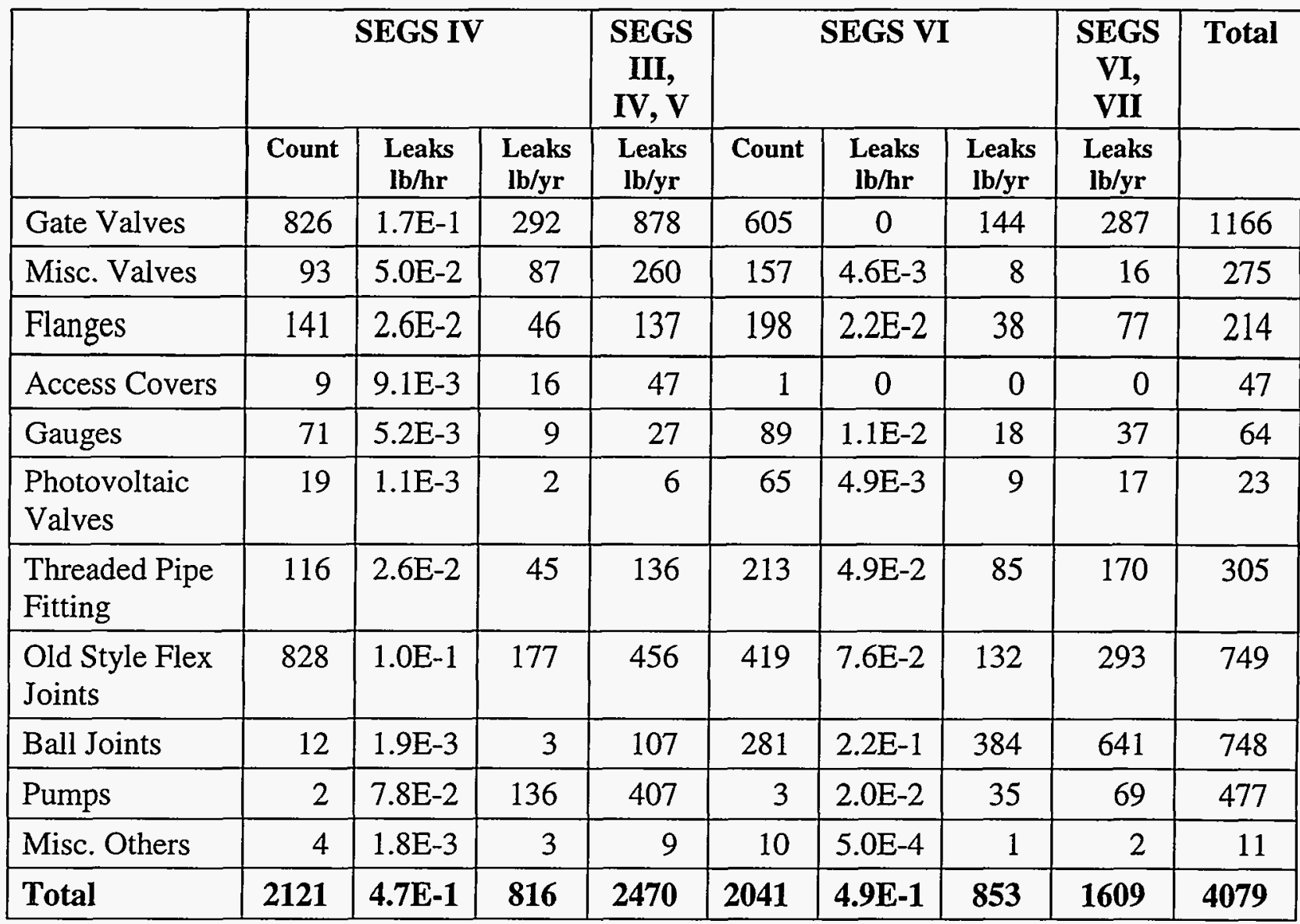




\title{
Appendix AA WATER USE STUDY
}

\section{Background}

\begin{abstract}
All site water is supplied by Antelope Valley East Kern Water Agency (AVEK). The water is provided via an 8.5-mile pipeline from the California aqueduct on a 30-year contract with a maximum annual supply of 2,250 acre-feet. The water is pumped via the pipeline into any of seven on-site raw water storage tanks, which combined have a maximum raw water storage capacity of 2,000,000 gallons.
\end{abstract}

The quality of AVEK water greatly affects the quantity of water required by the plants. Poor water quality (high conductivity) results in increased water use. AVEK water quality can vary greatly and in general varies significantly. Figure AA-1 shows the conductivity for the last five years. Increased conductivity affects site water balance in two ways. The higher conductivity levels limit the cycles of concentration in the cooling system, thus increasing the blowdown into the evaporation ponds to prevent scale buildup and/or corrosion. The second major effect of increased conductivity in the AVEK water is in the operation of the site demineralizers that produce treated water for condensate makeup and reflector panels cleaning. The higher conductivity of the supply water decreases the throughput of the demineralizers between regenerations. The variation in water quality from AVEK is largely because of weather cycles. Dry conditions in the California delta area cause increased solids pickup. This pattern is expected to be seasonal and varies from year to year. Note that 1996 and 1997 were low compared to 1992, 1993, and 1995, with a relatively steep rise in late 1997.

An empirical model to project future water quality was developed by studying the past seven years of AVEK water quality trends, weather patterns, and the relationship between the two. A "typical" year was established based on the trends from the previous seven years, while "typical" wet and dry years were also established to allow for seasonal changes. This model, used properly, matches actual data quite well. The primary purpose of the model is for budget and material (chemicals) purchase planning.

\section{Water Use}

A number of additional totalizer water meters were installed to determine the water requirements of the plants. Although data were available on the total amount of water purchased, the necessary meters were not in place to give a breakdown of where and when the water was used. Inlet totalizers were installed on the water supply lines to the cooling towers, demineralizers, condensate, and mirror-wash storage tanks. Meters were also installed in the cooling tower blowdown and the neutralization tank outlets. In addition, a flow indicator was installed in the cooling tower return lines at Solar Electric Generating Systems (SEGS) III and VI to gauge cooling tower performance. All new or existing meters will be hooked up to the new distributed control system (DCS) at SEGS III, IV, and V and to the existing DCS at SEGS VI and VII. Figure AA-2 is a schematic showing the locations of the water meters on the SEGS plants. 


\section{RAW WATER CONDUCTIVITY}

Actuals 1992-1997

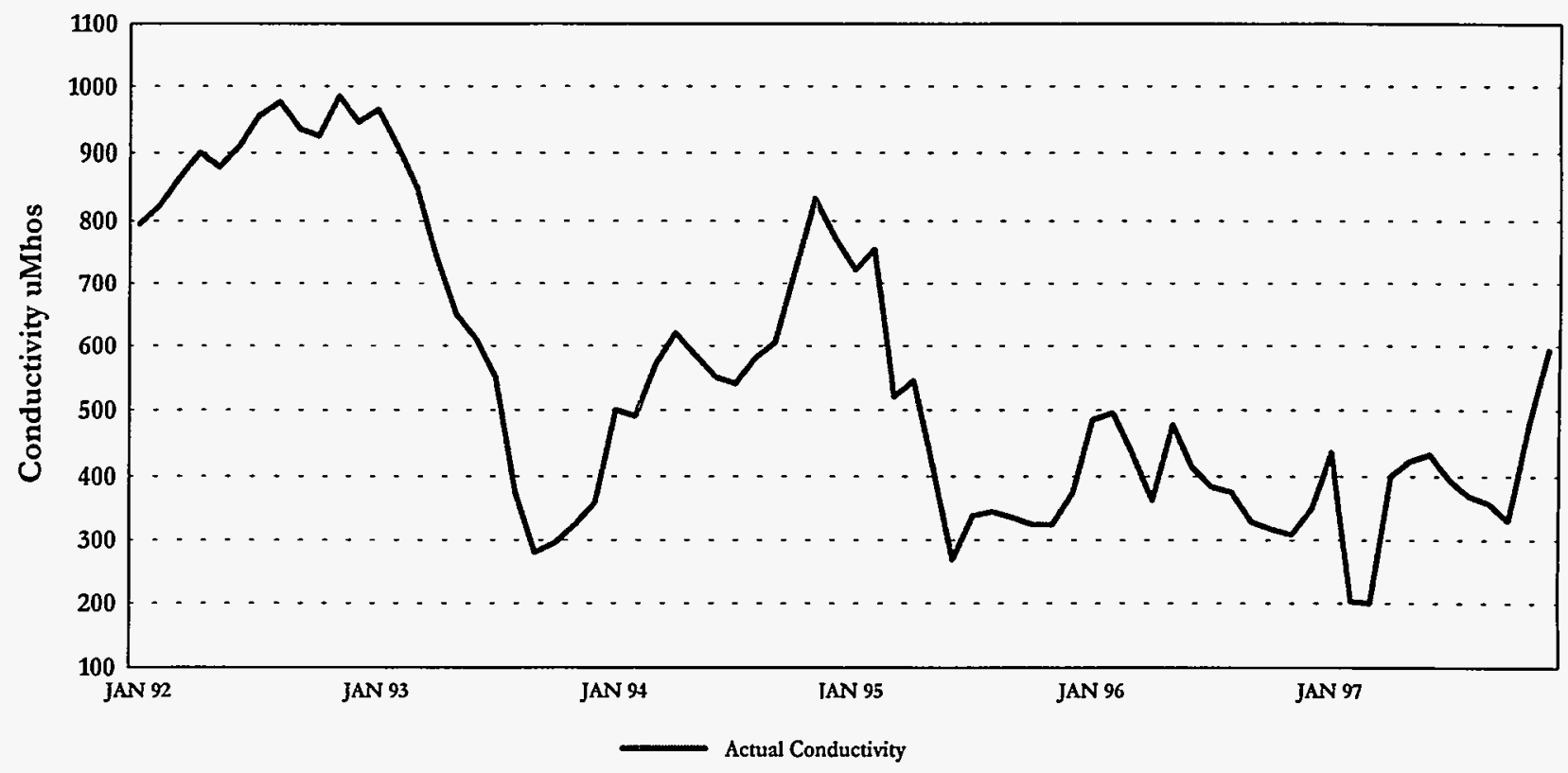

Figure AA-1. Raw water conductivity at Kramer Junction.

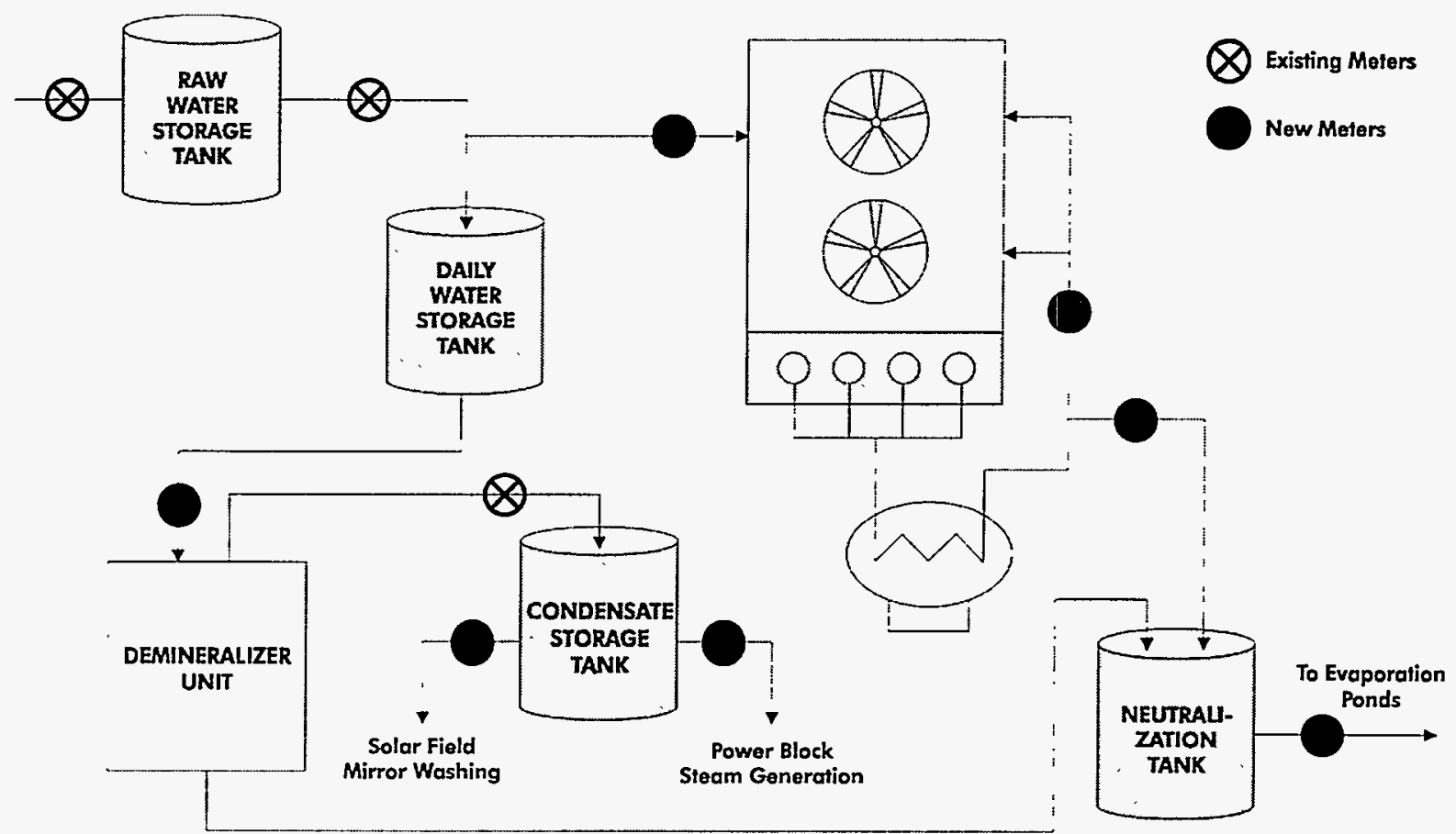

Figure AA-2. Water flow meter locations on the SEGS plants. 
A spreadsheet was developed to report and track the new metering data points. All water meter readings are logged into the OpLog@ program daily. The data include water treatment chemical inventory, raw water quality, and electrical production, which provide inputs to the model for the reporting of all water and chemical use. Two examples of the output are shown in Figure AA-3 and Table AA-1. Figure AA-3 shows an annual summary of water use for the entire Kramer Junction site, and Table AA-1 summarizes complete information on water and chemical use and costs for 1995, including a monthly breakdown. The total raw water cost in 1995 was about $\$ 450,000$.

This detailed reporting of water and chemical use data now allow operations and engineering to monitor use rates and expenses in order to identify abnormal use rates should they occur. It also allows evaluation of the effects of equipment modifications, if such actions are taken.

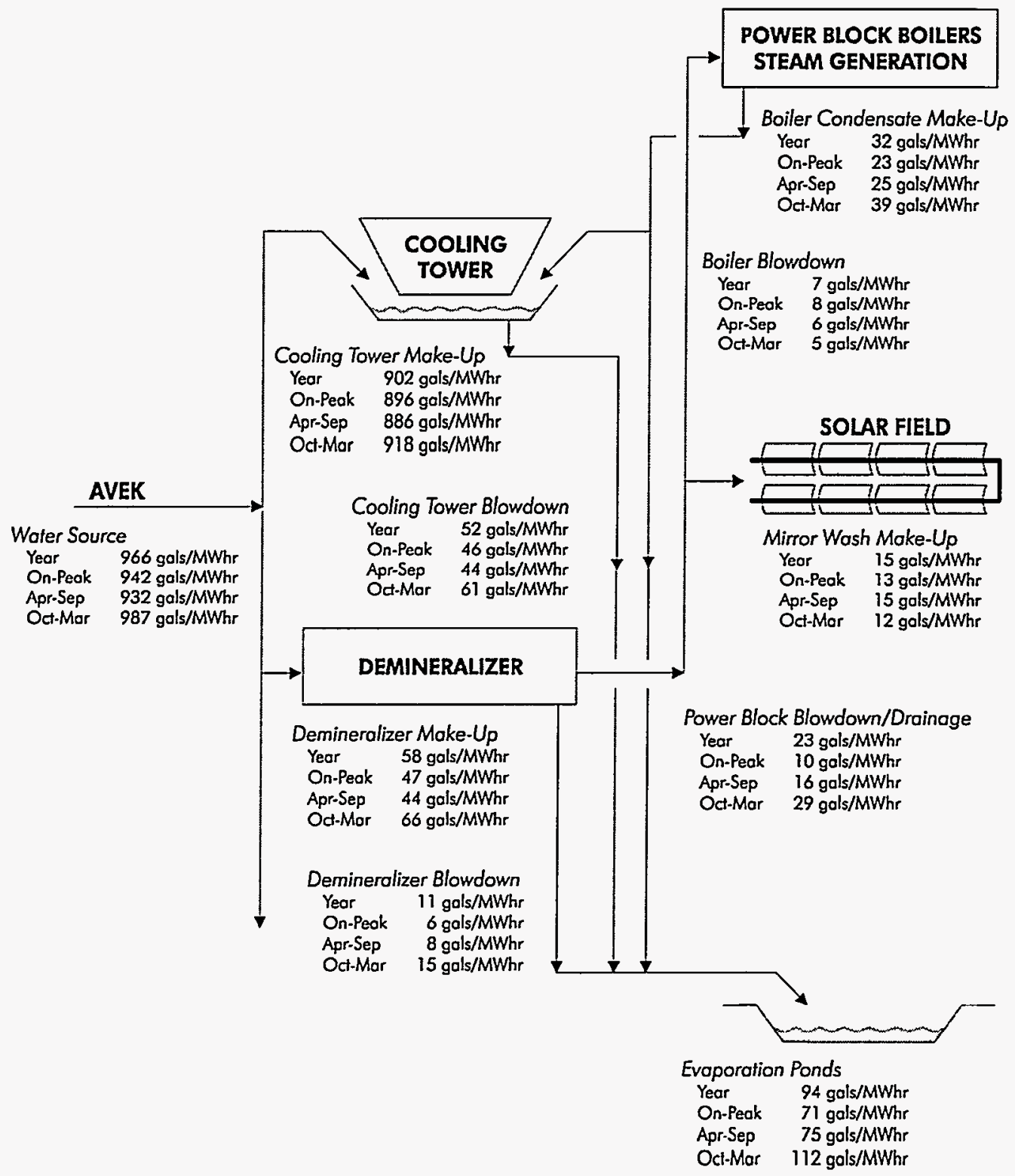

Figure AA-3. SEGS III through VII water use in gallons per megawatt hour. 


\section{Table AA-1. December 1995 Year-to-Date Water and Chemical Use Performance Summary}

Raw Water Usage

\begin{tabular}{|c|c|c|c|c|c|c|c|}
\hline & III & IV & $\mathbf{V}$ & VI & VII & Total & Total Avg. \\
\hline Raw Water Usage - gals & $81,172,900$ & $81,460,900$ & $91,410,100$ & $78,373,800$ & $74,360,500$ & $406,778,200$ & $81,355,640$ \\
\hline Raw Water - cost & $\$ 89, \$ 97$ & $\$ 89,915$ & $\$ 100,896$ & $\$ 86,507$ & $\$ 82,077$ & $\$ 448,992$ & $\$ 89,798$ \\
\hline Raw Water Used - gals/MWh & 973 & 1,007 & 983 & 833 & 833 & 923 & 923 \\
\hline
\end{tabular}

Water Usage Breakdown - Gallons

\begin{tabular}{|c|c|c|c|c|c|c|c|}
\hline & III & IV & $\mathbf{V}$ & VI & VII & Total & Total Avg. \\
\hline Cooling Tower make-up - gallons & $78,687,000$ & $77,888,000$ & $86,653,000$ & $70,861,000$ & $68,394,000$ & $382,483,000$ & $76,496,600$ \\
\hline Cooling Tower blowdown - gallons & $4,174,017$ & $5,449,291$ & $4,433,785$ & $4,377,826$ & $3,754,445$ & $22,189,364$ & $4,437,873$ \\
\hline Cooling Tower evap/drift - gallons & $74,512,983$ & $72,438,709$ & $82,219,215$ & $66,483,174$ & $64,639,555$ & $360,293,636$ & $72,058,727$ \\
\hline Cooling Tower evap/drift - \% & $94.7 \%$ & $93.0 \%$ & $94.9 \%$ & $93.8 \%$ & $94.5 \%$ & $94.2 \%$ & $94.2 \%$ \\
\hline Cooling Tower average cycles & 10.6 & 10.6 & 11.3 & 11.1 & 10.6 & 10.8 & 10.8 \\
\hline
\end{tabular}

\begin{tabular}{|c|c|c|c|c|c|c|c|}
\hline Demineralizer make-up - gallons & $3,689,700$ & $3,836,228$ & $4,910,591$ & $6,066,800$ & $4,855,250$ & $23,358,569$ & $4,671,714$ \\
\hline Condensate storage tank make-up - gallons & $1,422,300$ & $2,344,500$ & $1,977,300$ & $4,363,896$ & $3,182,594$ & $13,290,590$ & $2,658,118$ \\
\hline Mirror wash tank make-up - gallons & $1,004,900$ & $1,115,300$ & $1,306,500$ & $1,088,206$ & $1,162,270$ & $5,677,176$ & $1,135,435$ \\
\hline Demineralizer blowdown - gallons & $1,262,500$ & 376,428 & $1,626,791$ & 614,698 & 510,386 & $4,390,803$ & 878,161 \\
\hline Condensate losses - gallons & $1,432,300$ & $2,334,500$ & $1,979,300$ & $4,358,396$ & $3,178,594$ & $13,283,090$ & $2,656,618$ \\
\hline
\end{tabular}

Water Usage Breakdown - Gallons/MWh

\begin{tabular}{|c|c|c|c|c|c|c|c|}
\hline & III & IV & $\mathbf{v}$ & VI & VII & Total & Total Avg. \\
\hline Cooling Tower make-up - gallons/MWh & 944 & 963 & 932 & 753 & 766 & 868 & 868 \\
\hline Cooling Tower blowdown - gallons/MWh & 50 & 67 & 48 & 47 & 42 & 50 & \\
\hline Cooling Tower evap/drift - gallons/MWh & 894 & 896 & 884 & 706 & 724 & 818 & 818 \\
\hline
\end{tabular}

\begin{tabular}{|c|c|c|c|c|c|c|c|}
\hline Demineralizer make-up - gallons $/ \mathrm{MWh}$ & 44 & 47 & 53 & 64 & 54 & 53 & 53 \\
\hline Condensate storage tank make-up - gallons/MWh & 17 & 29 & 21 & 46 & 36 & 30 & 30 \\
\hline Mirror wash tank make-up - gallons/MWh & 12 & 14 & 14 & 12 & 13 & 13 & 13 \\
\hline Demineralizer blowdown - gallons/MWh & 15 & 5 & 17 & 7 & 6 & 10 & 10 \\
\hline
\end{tabular}

Cooling Water Chemical Treatment Usage Breakdown - Gallons

\begin{tabular}{|c|c|c|c|c|c|c|c|}
\hline & III & IV & $\mathbf{V}$ & VI & VII & Total & Total Avg. \\
\hline Cooling Tower acid makeup - gallons & 2,095 & 1,885 & 2,170 & 2,190 & 1,640 & 9,980 & 1,996 \\
\hline Cooling Tower Chlorine make-up - gallons & 4,405 & 4,405 & 4,520 & 4,095 & 4,000 & 21,425 & 4,285 \\
\hline Cooling Tower PO4 make-up - gallons & 579 & 632 & 687 & 572 & 571 & 3,041 & 608 \\
\hline Total Treatment Cost & $\$ 11,694$ & $\$ 12,241$ & $\$ 13,211$ & $\$ 11,464$ & $\$ 11,032$ & $\$ 59,642$ & $\$ 11,928$ \\
\hline
\end{tabular}

Condensate Chemical Treatment Usage Breakdown - Gallons

\begin{tabular}{|c|c|c|c|c|c|c|c|}
\hline & III & IV & $\mathbf{V}$ & VI & VII & Total & Total Avg. \\
\hline Demineralizer acid make-up - gallons & 4210 & 2570 & 5900 & 5350 & 4060 & 22090 & 4418 \\
\hline Demineralizer caustic make-up - gallons & 9220 & 5595 & 9705 & 10820 & 6855 & 42195 & 8439 \\
\hline Misc. PB chemicals - gallons & 380 & 380 & 417 & 397 & 388 & 1962 & 392.4 \\
\hline Total Treatment Cost & $\$ 13,726$ & $\$ 8,491$ & $\$ 15,423$ & $\$ 16,335$ & $\$ 10,931$ & $\$ 64,905$ & $\$ 12,981$ \\
\hline
\end{tabular}

Total Process Cost

\begin{tabular}{|c|c|c|c|c|c|c|c|}
\hline & III & IV & $\mathbf{v}$ & VI & VII & Total & Total Avg. \\
\hline Total process water cost & $\$ 115,016$ & $\$ 110,647$ & $\$ 129,530$ & $\$ 114,306$ & $\$ 104,041$ & $\$ 573,539$ & $\$ 114,708$ \\
\hline Total process water $\cos t / \mathrm{MWh}$ & $\$ 1.38$ & $\$ 1.37$ & $\$ 1.39$ & $\$ 1.21$ & $\$ 1.17$ & $\$ 1.30$ & $\$ 1.30$ \\
\hline Condensate - cost/gallon & $\$ 0.007$ & $\$ 0.004$ & $\$ 0.006$ & $\$ 0.004$ & $\$ 0.004$ & $\$ 0.005$ & $\$ 0.005$ \\
\hline
\end{tabular}




\section{Annual Data}

Figure AA-4 shows the normalized annual raw water in gallons per megawatt-hour (MWh) and the total annual gross electricity production for the years 1989 through 1997. Because raw water use is strongly tied to electrical production, the important characteristic is the normalized water use. The steady reduction in use is a result of better conservation practices at the plants as well as the effects of source water quality. While it is hard to accurately assess their relative impact, we estimate that about $30 \%$ to $40 \%$ of the improvement is a result of changes in operation and maintenance $(\mathrm{O} \& \mathrm{M})$ practices.

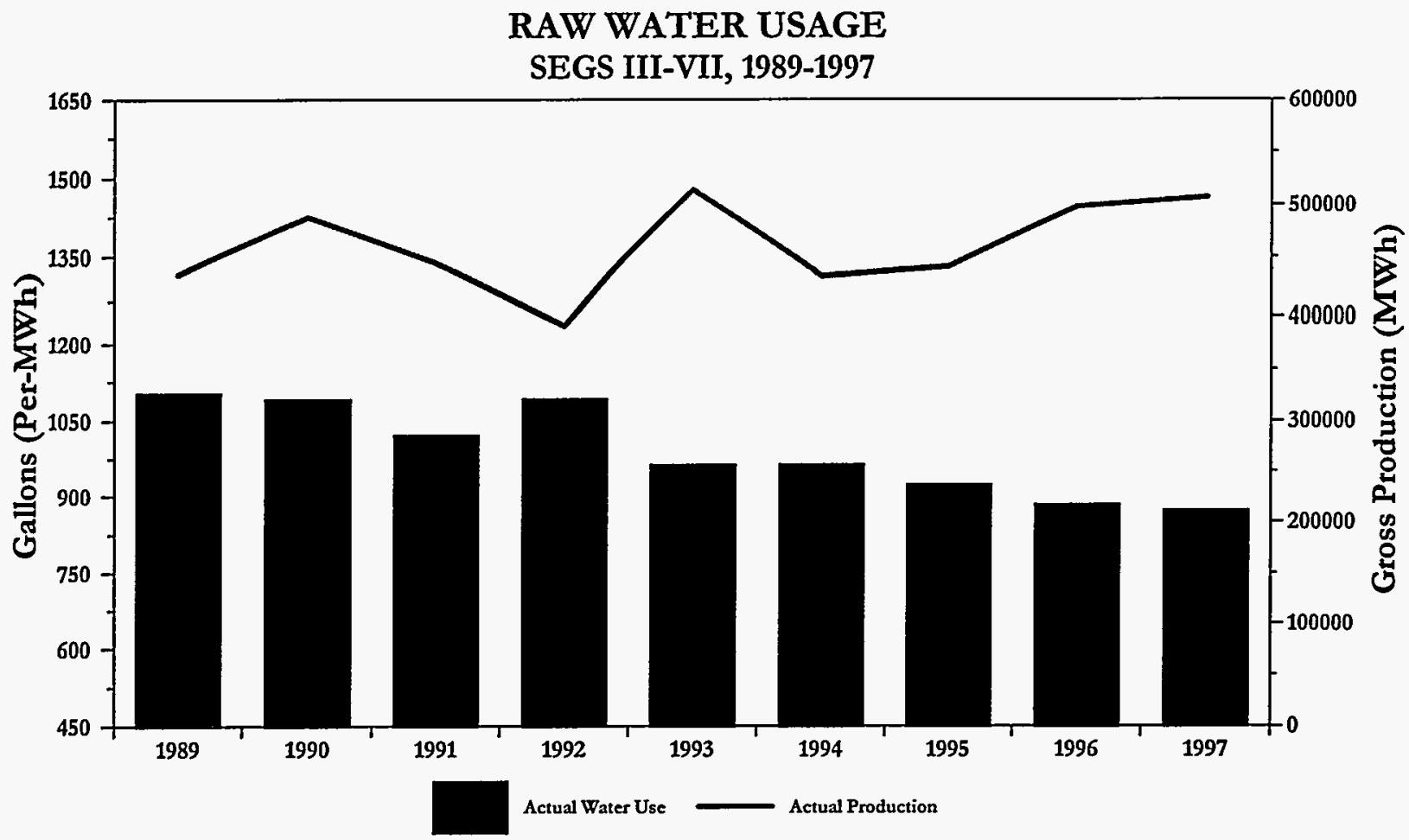

Figure AA-4. Raw water use versus gross electricity production for SEGS III through VII from 1989 through 1997.

\section{Examples of Improved Water Conservation Program}

The annual cost for raw water and chemicals is about $\$ 1$ million per year. Improved tracking of water use has permitted accurate billing of water and chemical use to each SEGS separately, and provides better insights into expected usage rates. For example, the detection of an error in the December 1997 bill from AVEK saved \$25.5 thousand for the plants.

Chemical use (and costs) is down, in large part due to better cooling tower and demineralizer operation. Much more attention can now be paid to raw water quality and the number of required concentration cycles in the site cooling towers. Having established acceptable water quality limits, the plants work closely with the chemical vendor to make adjustments for the most cost-effective chemical treatment. Equipment changes were made after accurate metering gave good water use data for each system. Measurements at the cooling tower showed that the drift was twice design values; investigation led to degraded nozzles, fill, drift eliminators, and 
louvers. Repairs were made, and instrumentation was added to allow automatic blowdown control, which reduced water use. Connecting control to the DCS system resulted in even tighter control and flexibility. Similarly, a partially completed and very detailed program of evaluation and adjustments on the demineralizers has led to higher quality condensate and cost reductions (36\% at SEGS III, $11 \%$ at SEGS VI, and $8 \%$ at SEGS VII).

In summary, this program combined with careful O\&M practices related to water use has been an important contributor to reduced O\&M costs at the sites. 


\section{Appendix AB RECOMMENDEd OpERATION AND MAINTENANCE PLAN}

\section{Applicable Hardware and System Configurations}

A large solar thermal plant typically consists of a solar field interfaced with a central conventional power technology. This description characterizes parabolic trough and central receiver/ heliostat plants rather than dish/Stirling systems in which the power system is distributed. The incremental solar operation and maintenance (O\&M) requirements for the centralized power system are those required by the solar field. For the case of parabolic troughs, for example, there is a clear distinction between the solar collector field and the remainder of the plant, consisting of the power island, conventional balance of plant, and the heat transport system. That is, the solar field is part of the solar steam system, which in addition to the solar field is comprised of the necessary pumps, vessels, and heat exchangers to convert collected solar thermal energy into turbine inlet steam. Maintenance of the latter, more conventional, equipment does not inherently require a larger organization than would normally be used for the conventional power island and balance of plant, though additional spare parts and consumable allotments are needed.

The solar field, on the other hand, is an extensive distributed array of concentrating collectors tracking the sun, which necessitate additional maintenance staff and spare parts. Two fundamental types of concentrating solar power plants using parabolic troughs have been either constructed or studied, one in which the solar steam system supplies steam to a conventional Rankine steam turbine cycle, and the second in which solar steam supplements the steam turbine inlet of a combined cycle (gas turbine/steam turbine) set. The solar field technology is identical for both.

\section{Plant O\&M Considerations}

There is no single optimal approach to the O\&M organization for conventional power plants. Variations in staffing and structure arise because of the management practices of the owners and the O\&M company, the particular design and layout of a given plant, labor practices and conditions at the plant, the degree of automation, and other important factors. For this reason, the discussion of O\&M staffing presented here is focused only on the solar field requirements and can be applied to either type of solar power plant.

\section{Solar Field O\&M Plan and Cost}

The plan was developed for a solar field size of $500,000 \mathrm{~m}^{2}$ of collector aperture area. For a moderately high insolation area, this size solar field is reasonable for an 80-MW solar electric generating system (SEGS)-type plant, though at peak solar conditions the solar field output could drive a $100+M W$ turbine. While the unit costs for staffing and materials would differ for smaller or larger facilities, these influences are relatively weak because of the modular nature of the solar field. 


\section{Staffing Level}

The plan is based on the experience and knowledge gained at the Kramer Junction, California, plants but does not mirror the exact staffing structure at that site. Rather, this plan is oriented toward a new facility that would fully benefit in the design and construction phases from the lessons learned at the SEGS plants. It also attempts to accommodate varying O\&M staffing philosophies by formulating staffing from nominal and reduced staff perspectives. It is very difficult, if not impossible, to relate specific O\&M structures and approaches to long-term O\&M costs. For example, an O\&M group with full predictive and preventive O\&M programs may save equipment costs in the end, but in the near term, O\&M costs would be higher. Furthermore, the needs, nature, and complexity of these programs are greatly influenced by the selection of and initial investment in the solar field components and equipment. In an attempt to accommodate different organizational perspectives, the nominal values reflect what we believe is the staffing plan that provides the most cost-effective trade-off between labor costs and equipment reliability. The reduced staffing, on the other hand, represents the minimum staffing level that we believe to be prudent, though "bare bones," for adequate solar field maintenance. Scenarios have been developed for developed and developing countries (see Tables AB-1 and $\mathrm{AB}-2)$.

The solar field is characterized by thousands of individual parts in modular solar collector assemblies or heliostats. Management of O\&M requires good tracking tools and a dedicated staff. Such tools have been developed as part of the joint Sandia National Laboratories/KJC Operating Company program and, combined with a comprehensive maintenance planning and control system, provide the means to maintain a reliable, high-performance solar field.

In the nominal staffing scenario, management and supervision is centered within the solar field maintenance organization, and skilled personnel are assigned to conduct expedient maintenance and mirror washing. As a note of clarification, the primary responsibility of "field operators" is to monitor, in considerable detail, the condition and repair needs in the solar field.

The reduced staffing scenario, on the other hand, relies on partial top-level management from the conventional O\&M organization and puts more emphasis on the supervisory roles in the solar field group. Less staff will result in slower problem identification, slower response times and, presumably, a lower reliability.

It is very difficult to generalize the requirements in developing countries, where typically productivity and salaries are lower and the staff assigned to tasks can be considerably larger. In an attempt to capture this expectation, staffing levels have been increased, as shown in Table AB-2. A dedicated solar field manager is retained for both scenarios. In the reduced mode, the maintenance supervisor will also take responsibility for the mirror wash crew. Salary levels have been reduced to $30 \%$ of those in developed countries.

\section{Material Costs}

Materials consist of replacement parts, consumables (dominated by water for mirror washing and heat transfer fluid [HTF] replacement), and tools. The following list shows the major replacement parts, prices, and assumed annual replacement rate in terms of percent of total. Minor spares and tools are captured in the miscellaneous category. Mirror-washing water and HTF are listed as consumables. In a new well-designed plant, HTF makeup will be very low, as is 
presently the case at Kramer Junction. The unit cost for HTF makeup listed in Table AB-3 is the total cost of the initial HTF fluid at $\$ 12$ per gallon.

Water costs for the nominal case represent Kramer Junction costs, where raw water is purchased from the California aqueduct system. Less expensive (cheap) water is assumed for the reduced cost case at a 75\% cost reduction. For developing countries, it is assumed that reliability is lower and spare parts costs will increase by $30 \%$.

Table AB-1. Staffing in Developed Countries

Staffing: Developed Countries (eg, US., Europe)

For SF size $=500000 \mathrm{me} \quad$ OH rate

\begin{tabular}{|c|c|c|c|c|c|c|c|}
\hline \multirow[b]{2}{*}{ Solar Field Manager } & $\begin{array}{c}\text { Namine } \\
\#\end{array}$ & $\begin{array}{c}\text { Rediced } \\
\text { \# }\end{array}$ & Leve & $\begin{array}{l}\text { Rade, } \$ \text { hy } \\
\text { Direct }\end{array}$ & $\begin{array}{c}35 \% \\
\text { Burdened }\end{array}$ & $\begin{array}{l}\text { Arnul \$K } \\
\text { Nonina }\end{array}$ & Reduced \\
\hline & $\overline{1}$ & 0 & Serior & 50 & 67.5 & 67.5 & 0.0 \\
\hline Maintenance Supervisor & 1 & 1 & Stilled & 45 & 60.8 & 60.8 & 60.8 \\
\hline 2stifts Welder & 1 & 1 & Skilled & 40 & 54.0 & 108.0 & 54.0 \\
\hline \multirow[t]{2}{*}{ (4 10h days) } & 2 & 1 & Experd & 35 & 47.3 & 189.0 & 47.3 \\
\hline & 1 & 1 & Skilled & 40 & 54.0 & 108.0 & 54.0 \\
\hline Lead Mrror Wash Spervisor & 1 & 1 & Stilled & 45 & 60.8 & 60.8 & 60.8 \\
\hline 1 shift Equip. Opers. & 4 & 2 & Experd & 35 & 47.3 & 189.0 & 94.5 \\
\hline \multirow[t]{2}{*}{ Field Operator (status) } & 5 & 3 & Experd & 30 & 40.5 & 2025 & 121.5 \\
\hline & 20 & 13 & & & Total & 985.5 & 4928 \\
\hline
\end{tabular}

Table AB-2. Staffing in Developing Countries

Staffing: Developing Cantries

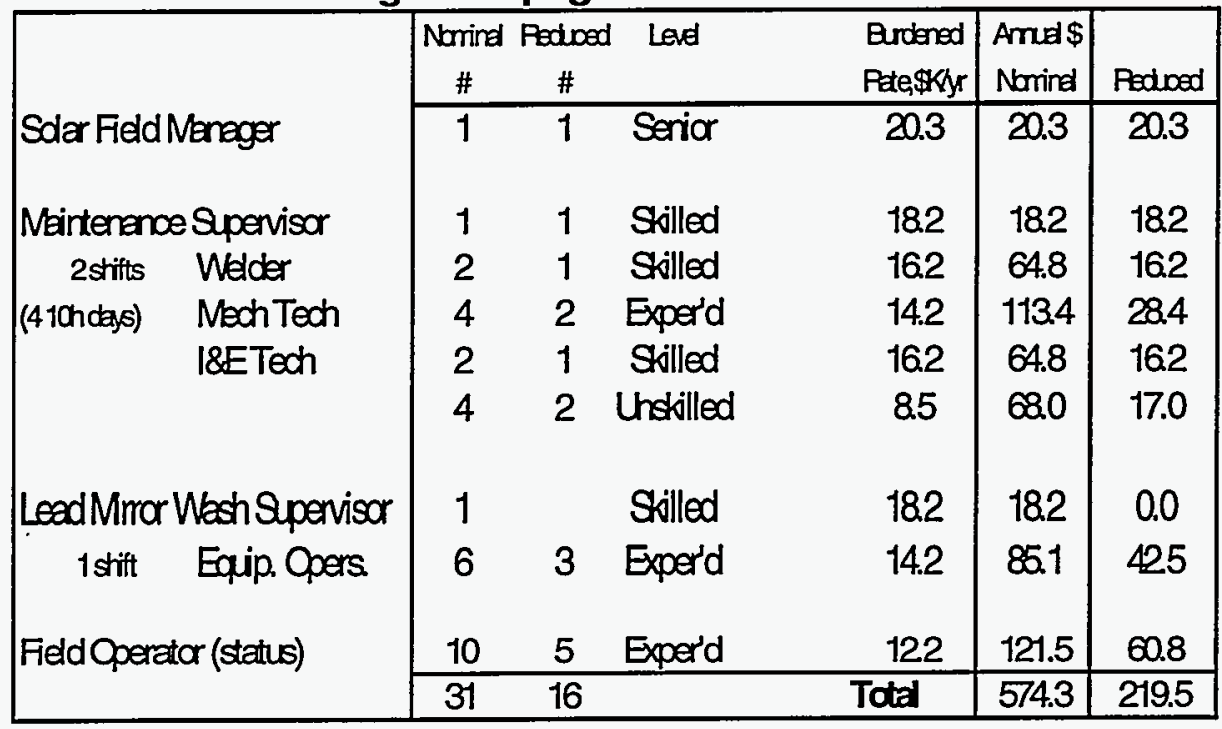




\section{Specialized Equipment}

Solar field maintenance is more efficient and cost-effective if certain specialized equipment is used. The equipment listed in Table AB-4 is primarily used for mirror washing, HCE replacement, and solar field monitoring. The data listed is for a developed country. In a developing country, it is assumed that less specialized equipment will be used, reducing equipment costs by $30 \%$.

Equipment life is assumed to be five years, and the annual straight-line cost is carried to the aggregate $O \& M$ cost calculation.

\section{Annual O\&M Cost}

The annual costs for staff, spare parts, and equipment are added in Table AB-5 for the four cases considered here.

Both the total and unit annual costs are shown for nominal and reduced plans in developed and developing countries. Results for solar field O\&M costs vary from $0.75 \phi$ to $1.16 \phi / \mathrm{kWh}$. Given the many assumptions, uncertainty levels are relatively high, at least $25 \%$. Therefore, it is reasonable to state that this analysis generally shows an incremental O\&M cost for the solar field in a solar thermal power plant of $1 ф / \mathrm{kWh}$. Costs will scale weakly and inversely with field size.

Table AB-3. Total Cost of Parts and Materials

\begin{tabular}{|c|c|c|c|c|c|}
\hline \multicolumn{6}{|c|}{ Parts and Materials } \\
\hline & $\frac{\text { Unit Cost }}{\$}$ & $\%$ replace & $\begin{array}{l}\text { SF size= } \\
\text { m2/unit }\end{array}$ & $\begin{array}{l}\mathrm{m} 2 \\
\$ / \mathrm{m} 2-\mathrm{yr}\end{array}$ & $\$ K / y r$ \\
\hline Mirrors & 100 & 0.5 & 2 & 0.250 & 125.0 \\
\hline HCEs & 700 & 3.0 & 22 & 0.963 & 481.7 \\
\hline Sun Sensor & 150 & 0.5 & 545 & 0.001 & 0.7 \\
\hline LOCs & 200 & 0.5 & 545 & 0.002 & 0.9 \\
\hline Ball joints & 2100 & 0.5 & 273 & 0.039 & 19.3 \\
\hline Hdr. Drive & 6000 & 0.5 & 545 & 0.055 & 27.5 \\
\hline Miscellaneous & \multicolumn{4}{|c|}{ assumed as $5 \%$ of total equipment costs above } & 33.6 \\
\hline HTF Pump Seals & 1200 & 2 per year & 500000 & 0.005 & 2.4 \\
\hline HTF Makeup & $4,221,423$ & 1 & 500000 & 0.084 & 42.2 \\
\hline Water & \multicolumn{4}{|c|}{ demineralized water for mirror washing } & 243.3 \\
\hline \multicolumn{5}{|c|}{$\begin{array}{r}\text { With } 30 \% \text { higher material costs and cheap water } \\
\text { With only cheap water } \\
\text { With only higher material costs }\end{array}$} & $\begin{array}{r}976.5 \\
1034.9 \\
810.1 \\
1196.5\end{array}$ \\
\hline
\end{tabular}


Table AB-4. Equipment List

\begin{tabular}{|c|c|c|c|c|}
\hline \multicolumn{5}{|c|}{ Specialized Equipment } \\
\hline \multirow{6}{*}{\begin{tabular}{l} 
Dump truck \\
SF operator vehicle \\
HTF Evacuation rig \\
Mirror wash rigs - Twister \\
\multicolumn{1}{c}{ - Deluge }
\end{tabular}} & Cost,\$K & \# & $\$ / \mathrm{m} 2$ & \\
\hline & 30 & $\overline{1}$ & 0.06 & \\
\hline & 20 & 3 & 0.12 & \\
\hline & 45 & 1 & 0.09 & \\
\hline & 100 & 2 & 0.40 & \\
\hline & 30 & 1 & 0.06 & \\
\hline \multirow{5}{*}{$\begin{array}{l}\text { HTF cooler rig } \\
\text { HCE trailer } \\
\text { HCE installation rig } \\
\text { RP container carrier } \\
\text { Lube trailer }\end{array}$} & 30 & 1 & 0.06 & \\
\hline & 15 & 2 & 0.06 & \\
\hline & 20 & 1 & 0.04 & \\
\hline & 17 & 1 & 0.03 & \\
\hline & 6 & 1 & 0.01 & \\
\hline \multirow[t]{4}{*}{ Tractor } & 20 & 1 & 0.04 & $\$ \mathrm{~K}$ \\
\hline & \multicolumn{3}{|c|}{$\begin{array}{ll}\text { Total } & 0.98 \\
5 & \text { yrs }=\end{array}$} & 488 \\
\hline & over & 5 & & 97.6 \\
\hline & \multicolumn{3}{|c|}{ With reduced equip costs: } & 68.3 \\
\hline
\end{tabular}

Table AB-5. Total Annual O\&M Costs

\begin{tabular}{|c|c|c|c|c|c|c|c|}
\hline \multicolumn{8}{|c|}{ Annual Solar Field O\&M Cost, cents/kWh } \\
\hline \multirow{8}{*}{$\begin{array}{l}\text { SF size } \\
\text { DNI } \\
\text { SF thermal effic. } \\
\text { PB cycle effic. } \\
\text { Annual MWh } \\
\text { Annual Cost, } \$ K\end{array}$} & $\overline{\mathrm{m} 2}$ & & 500,000 & & & & \\
\hline & $\mathrm{kWh} / \mathrm{m} 2-\mathrm{yr}$ & & 2300 & & & & \\
\hline & 1 & & 0.50 & & & & \\
\hline & 1 & net & 0.31 & & & & \\
\hline & & & 176985 & Develope & Countries & Developir & Countries \\
\hline & & & Annual O\&M, \$K & 2059.6 & 1400.5 & 1839.1 & 1322.7 \\
\hline & & & Cents/kWh & 1.16 & 0.79 & 1.04 & 0.75 \\
\hline & & & & Nominal & Reduced & Nominal & Reduced \\
\hline
\end{tabular}


This page intentionally left blank. 


\section{Distribution}

1 Jim Blackmon

Boeing North American

689 Discovery Drive

Huntsville, AL 35806

1 Ernie Palomino

Salt River Project

Research \& Development

P.O. Box 52025

Mail Station ISB664

Phoenix, AZ 85072-2025

1 Harry Braun

Stirling Energy Systems

6245 North 24th Parkway

Phoenix, AZ 85016

1 Scott McLellan

Arizona Public Service Co.

P.O. Box 53933

MS 3888

Phoenix, AZ 85072-3933

1 Bob Hess

Salt River Project

Power Generation Services

P.O. Box 52025

Mail Station POB004

Phoenix, AZ 85072-2025

1 Jim Augustyn

1029 Solano Avenue

Suite C

Albany, CA 94706

1 Robert Cable

KJC Operating Company

41100 Highway 395

Boron, CA 93516

10 Scott Frier

KJC Operating Company

41100 Highway 395

Boron, CA 93516

1 Bob Litwin

Boeing North American

Rocketdyne Division

6633 Canoga Avenue

P.O. Box 7922 - Mail Code LA38

Canoga Park, CA 91309-7922
1 Larry Hamlin

Southern California Edison Co.

Solar Two Project

37110 E. Santa Fe Street

P.O. Box 307

Daggett, CA 92327-0307

1 Art Romero

Energy Services Inc.

Solar Two Project

37110 E. Santa Fe Street

P.O. Box 307

Daggett, CA 92327-0307

1 Judd Kilimnik

Energy Services Inc.

Solar Two Project

37110 E. Santa Fe Street

P.O. Box 307

Daggett, CA 92327-0307

1 Michael Echimendy

Energy Services Inc.

Solar Two Project

37110 E. Santa Fe Street

P.O. Box 307

Daggett, CA 92327-0307

$1 \quad$ Eric Wills

Daggett Leasing Corporation

35100 Santa Fe Street

P.O. Box 373

Daggett, CA 92327

5 David W. Kearney

Kearney \& Associates

14022 Condessa Drive

Del Mar, CA 92014

1 Byron J. Washom

Spencer Management Associates

P.O. Box 724

Diablo, CA 94528-0724

$1 \quad$ Mac Moore

BP Solar Inc.

2300 N. Watney Way

Fairfield, CA 94533 
Jan Hansen

UC Operating Services 43880 Harper Lake Road

Hinkley, CA 92347

$1 \quad$ Ken Stone

Boeing North American

5301 Bolsa Avenue

M/S 8011-C114

Huntington Beach, CA 92647

1 Joseph J. Iannucci

Distributed Utility Associates

1062 Concannon Boulevard

Livermore, CA 94550

1 Jerry Berquist

Southern California Edison Co.

690 Studebaker Road

Long Beach, CA 90803

1 Daryl Yonamine

Los Angeles Dept. of Water and Power

Alternate Energy Systems

111 North Hope Street, Rm. 661A

Los Angeles, CA 90012

$1 \quad$ Ed DeMeo

Electric Power Research Institute

P.O. Box 10412

3412 Hillview Avenue

Palo Alto, CA 94303

1 William B. Stine

California State Polytechnic University

Department of Mechanical Engineering

3801 West Temple Avenue

Pomona, CA 91768-4062

1 Alexander Jenkins

California Energy Commission

Energy Technology Development Division

R\&D Office

15169 th Street

MS-43

Sacramento, CA 95814-5512
1 Don Osborne

Sacramento Municipal Utility District

Generation Systems Planning

Power Systems Dept.

6201 'S' Street

P.O. Box 15830

Sacramento, CA 95852-1830

1 Bud Beebe

Sacramento Municipal Utility District

Generation Systems Planning

Power Systems Dept.

6201 'S' Street

P.O. Box 15830

Sacramento, CA 95852-1830

1 Barry L. Butler

Science Applications International Corp.

Room 2043, M/S C2J

10260 Campus Point Drive

San Diego, CA 92121

$1 \quad$ William Gould

Bechtel Corporation

50 Beale Street

50/19/B35

P.O. Box 193965

San Francisco, CA 94119-3965

$1 \quad$ Bruce Kelly

Bechtel Corporation

50 Beale Street

$50 / 15 \mathrm{D} 25$

P.O. Box 193965

San Francisco, CA 94119-3965

1 Ray Dracker

Bechtel Corporation

P.O. Box 193965

San Francisco, CA 94119-3965

$1 \quad$ David Elpern

AlliedSignal Power Systems Inc.

2525 W. 190th Street

Torrance, CA 90504-6099 
J. Borghese

Allied-Signal Aerospace Company

AiResearch, Los Angeles Division

Dept. 93055, M/S T-41

2525 West 190th Street

Torrance, CA 90509-2960

1 Donald Aitken

Union of Concerned Scientists

20100 Skyline Boulevard

Woodside, CA 94062

1 Stanley Hightower

U.S. Bureau of Reclamation

Code D-3710

P.O. Box 205007

Denver, CO 80225

6 National Renewable Energy Laboratory

Attn: Tom Williams

Hank Price

Mary Jane Hale

Al Lewandowski

Mark Mehos

Gary Nakarado

1617 Cole Boulevard

Golden, CO 80401-3393

1 Kelly Beninga

Science Applications International Corp.

15000 W. 6th Avenue

Suite 202

Golden, CO 80401

1 Randy Gee

Industrial Solar Technology

4420 McIntyre Street

Golden, CO 80403

1 Bob Martin

U.S. Department of Energy

Golden Field Office

1617 Cole Boulevard

Golden, CO 80401

1 Dave Gorman

Advanced Thermal Systems, Inc.

5031 W. Red Rock Drive

Larkspur, CO 80118
$1 \quad$ Glenn Strahs

U.S. Department of Energy

EE-13

Forrestal Building

1000 Independence Avenue, SW

Washington, DC 20585

5 Gary Burch

U.S. Department of Energy

EE-13

Forrestal Building

1000 Independence Avenue, SW

Washington, DC 20585

1 Ernesto N. Terrado

The World Bank

1818 H Street, NW

Washington, DC 20433

1 Charles Feinstein

World Bank

Chief, Global Climate Change Unit

1818 H Street, NW

Washington, DC 20433

1 Tom Rueckert

U.S. Department of Energy

EE-13

Forrestal Building

1000 Independence Avenue, SW

Washington, DC 20585

1 Steve McAllister

Solar Energy Industries Association

122 C Street, NW

4th Floor

Washington, DC 20001-2109

1 Charles W. Linderman

Edison Electric Institute

701 Pennsylvania Avenue, NW

Washington, DC 20004-2696

1 Magdalena Manzo

Sr. Operations Officer

Energy Operation Division

The World Bank

1818 H Street, NW

Washington, DC 20433 
1 Scott Sklar

Solar Energy Industries Association

122 C Street, NW

4th Floor

Washington, DC 20001-2109

1 Richard Spencer

World Bank

1818 H Street, NW

Washington, DC 20433

1 David Block

Florida Solar Energy Center

1679 Clearlake Road

Cocoa, FL 32922-5703

$1 \quad$ R.S. Allan

Florida Power and Light

P.O. Box 14000

Juno Beach, FL 33408

1 Luis Luzuriaga

5106 Brookeway Drive

Bethesda, MD 20816

$1 \quad$ G. Braun

Solarex

630 Solarex Court

Frederick, MD 21703

1 Dinesh Kumar

Princeton Economic Research Inc.

1700 Rockville Pike

Suite 550

Rockville, MD 20852

$1 \quad$ Allan L. Frank

The Solar Letter

9124 Bradford Road

Silver Spring, MD 20901-4918

1 Lennart Johansson

Stirling Thermal Motors

275 Metty Drive

Ann Arbor, MI 48103

5

Gilbert E. Cohen

Duke Solar

850 West Morgan Street

Raleigh, NC 27603
1 Richard Cole

New Mexico R\&D Forum

University of New Mexico

R\&D Communication Office

Albuquerque, NM 87131-6076

1 James Cannon

State of New Mexico

Energy Information Services

Energy, Minerals and Natural Resources

Department

2040 S. Pacheco Street

Santa Fe, NM 87505

1 Eric Dominguez

Nevada Power Co.

P.O. Box 230

Las Vegas, NV 89151

1 Rose McKinney-James

Corporation for Solar Technology and Renewables Resources

6863 West Charleston

Las Vegas, NV 89102

1 Bob Boehm

University of Nevada at Las Vegas

Dept. of Mechanical Engineering

4505 Maryland Parkway

P.O. Box 454027

Las Vegas, NV 89154-4027

1 W. Beale

Sunpower, Inc.

P.O. Box 2625

Athens, $\mathrm{OH}$ 45701-2627

1 Donald Ernst

Thermacore, Inc.

780 Eden Road

Lancaster, PA 17601

1 Michael Sloan

Sloan Engineering

4306 Ramsey Avenue

Austin, TX 78756

1 David White

Solar Kinetics, Inc.

10635 King William Drive

P.O. Box 540636

Dallas, TX 75354-0636 
1 C. Chadwick Small

Amoco/Enron Solar Power Development 1400 Smith Street

Houston, TX 77002-7361

1 Lorin Vant-Hull

University of Houston

Physics Department 5506

4800 Calhoun Road

Houston, TX 77204-5506

1 Tracy A. Reid

Amoco/Enron Solar Power Development 1400 Smith Street

Houston, TX 77002-7361

1 Ian Andrews

PacifiCorp

Utah Power Generation Engineering

1407 West North Temple

Salt Lake City, UT 84140-0001

1 Chris Rovero

Winrock International

1611 N. Kent Street

Alexandria, VA 22209

1 Stephen Kaneff

Australian National University

Energy Research Centre

Canberra ACT 0200

AUSTRALIA

1 Wolfgang Meike

Power and Water Authority

GPO Box 1921

Darwin NT 0801

AUSTRALIA

$1 \quad$ Dr. David Mills

Department of Applied Physics

Building A28

University of Sydney

Broadway, New South Wales 2006

AUSTRALIA

$1 \quad$ Wesley Stein

Pacific Power

Renewable Technology Development

P.O. Box 5257, GPO 2001

Sydney

AUSTRALIA
1 Evandro Sergio Camelo Cavalcanti

CEPEL

P.O. Box 68007

Rio de Janeiro - RJ

BRAZIL

$1 \quad$ Li Jungfeng

Center for Renewable Energy Development

State Planning Commission

Zhansimen Road

Shahe, Changping County

Beijing 102206

CHINA

1 Sami Zannoun

NREA

P.O. Box 4544 Massakin Dobat Elsaff

Hay Sades, Nasr City

Cairo

EGYPT

1 Adel Tawfik Soliman, Ph.D.

New \& Renewable Energy Authority

P.O. Box 39 El-Souk El-Togary

Maadi 11693 Cairo

EGYPT

1 Francois Pharabod

CNRS Programme Ecotech

Energy \& Environment

1 rue du Cerf

F-92195 Meudon

FRANCE

1 Helmut Ernst

KFW

Palmengartenstrasse 5-9

60325 Frankfurt am Main

GERMANY

$1 \quad$ Wilfried Grasse

SolarPACES

Kleimannsruh 7

D-38518 Gifhorn-Winkel

GERMANY

1 Manfred Boehmer

DLR - Köln MD - ET

Linder Hohe

P.O. Box 906058

D-51147 Köln

GERMANY 
1 Manfred Becker

DLR - Köln MD - ET

Linder Hohe

P.O. Box 906058

D-51140 Köln

GERMANY

1 Petr Svoboda

Pilkington Solar International GmbH

Muhlengasse 7

D-50667 Köln

GERMANY

1 Robert Pitz-Paal

DLR - Köln MD - ET

Linder Hohe

P.O. Box 906058

D-51147 Köln

GERMANY

1 Klaus Hennecke

DLR - Köln MD - ET

Linder Hohe

P.O. Box 906058

D-51147 Köln

GERMANY

1 Gerd Eisenbeiss

DLR - Köln PD - E

Linder Hohe

P.O. Box 906058

D-51140 Köln

GERMANY

2 Paul Nava and Josef Langenkamp

Pilkington Solar International GmbH

Muhlengasse 7

D-50667 Köln

GERMANY

1 Wolfgang Meinecke

DLR - Köln MD - ET

Linder Hohe

P.O. Box 906058

D-51147 Köln

GERMANY

1 Georg Brakmann

Fichtner

Sarweystrabe 3

70191 Stuttgart

P.O. Box 101454

70013 Stuttgart

GERMANY
$1 \quad$ Frank Lippke

Zentrum fur Sonnenenergie-und Wasserstoff-

Forschung

Hessbruehlstr.21

D-70565 Stuttgart

GERMANY

1 Wolfgang Schiel

SBP - Schlaich, Bergermann und Partner

Hohenzollernstr. 1

D-70178 Stuttgart

GERMANY

$1 \quad$ Reiner Buck

DLR - Stuttgart EN - TT

Pfaffenwaldring 38-40

D-70569 Stuttgart

GERMANY

1 Dr. Franz Trieb

Systems Analysis Group, Energy Division

Deutsche Forschungsanstalt fuer Luft und Raumfahrt

Pfaffenwaldring 38-40,

70569 Stuttgart

GERMANY

$1 \quad$ Ajit Gupta

Ministry of Non-Conventional Energy Sources

Block No. 14

CGO Complex

Lodhi Road

New Delhi 110003

INDIA

1 Radhey S. Sharma

Ministry of Non-Conventional Energy Sources

Block No. 14

CGO Complex

Lodhi Road

New Delhi 110003

INDIA

1 Dr. E. V. R. Sastry

Ministry of Non-Conventional Energy Sources

Block No. 14

CGO Complex

Lodhi Road

New Delhi 110003

INDIA 
J. B. S. Girdher

Ministry of Non-Conventional Energy Sources

Block No. 14

CGO Complex

Lodhi Road

New Delhi 110003

INDIA

1 Aharon Roy

Ben Gurion University of the Negev

Dept. of Chemical Engineering

P.O.B. 653

Beer Sheva 84105

ISRAEL

1 R. Biran

SOLEL

P.O. Box 23577

IS-91234 Jerusalem

ISRAEL

1 Avi Brenmiller

SOLEL

P. O. Box 45033

Jerusalem 91450

ISRAEL

1 Amnon Yogev

Weizmann Institute of Science

P.O. Box 26

Energy Research Dept.

IS-76100 Rehovot

ISRAEL

$1 \quad$ Michael Epstein

Weizmann Institute of Science

P.O. Box 26

IS-76100 Rehovot

ISRAEL

1 Dr. Anhar Hegazi

United Nation Economic and Social

Commission of Western Asia (UNESCWA)

Chief Energy Issues Section

Energy, Natural Resources \& Environmental Division

P.O. Box 927115

Amman

JORDAN
1 Gilles Flamant

CNRS - Centre National de la Recherche Scientifique

B.P. 5 Odeillo

66120 Font-Romeu

FRANCE

1 Roberto Cadenas

CFE

Calle Alejandro Volta No. 655

Col. Electricistas

C.P. 58290

Morelia, Michoacan

MEXICO

1 J.M. Huacuz

Instituto de Investigaciones Electricas

P.O. Box 1-475

62001 Cuernavaca, Morelos

MEXICO

1 Oleg S. Popel

IVTAN Institute for High Temperatures

Russian Academy of Sciences

Izhorskaya str. 13/19

Moscow 127412

RUSSIA

1 Irving Spiewak

European Commission Joint Research Centre

Institute for Prospective Technological Studies

W.T.C. Isla de la Cartaja

E-41092 Sevilla

SPAIN

1 Michael Geyer

DLR - PSA

Apartado 39

E-04200 Tabernas, Almería

SPAIN

1 Peter Heller

DLR - PSA

Apartado 39

E-04200 Tabernas, Almería

SPAIN

1 Manuel J. Blanco Muriel

CIEMAT - PSA

Apartado 22

E-04200 Tabernas, Almería

SPAIN 
Apartado 22

E-04200 Tabernas, Almería

SPAIN

1 Antonio Esteban Garmendia Inabensa

Manuel Velasco Pando, 7

41007 Sevilla

SPAIN

$1 \quad$ Frank Schillig

CIEMAT - PSA

Apartado 22

E-04200 Tabernas, Almería

SPAIN

1 Paul Kesselring

Paul Scherrer Institut

CH-5232 Villigen PSI

SWITZERLAND

1 Hans W. Fricker

FC Consulting

Breitestr. 22

CH-8544 Rickenbach

SWITZERLAND

1 Isoroku (Rocky) Kubo

Kombassan Holding AS

PK 159

Alayna 07400

TURKEY

1 Adrian Gaye

Solargen

Bartholomews

Foxton

Cambridge CB2 6SU

UNITED KINGDOM 